\title{
Role of Adhesion Proteins Neuroligin 2 and IgSF9b in the Amygdala Anxiety Circuitry
}

\section{PhD Thesis}

\author{
in partial fulfilment of the requirements \\ for the degree "Doctor rerum naturalium" \\ in the Neuroscience Program \\ at the Georg August University Göttingen, \\ Faculty of Biology
}

submitted by

\section{Olga Babaev}

born in

Vladimir, Russia

Göttingen, 2017 



\section{Thesis Committee}

Prof. Dr. Nils Brose (first reviewer)

Department of Molecular Neurobiology, Max Planck Institute of Experimental Medicine

Dr. Camin Dean (second reviewer)

Trans-synaptic signaling group, European Neuroscience Institute

Prof. Dr. Dr. Hannelore Ehrenreich

Clinical Neuroscience, Max Planck Institute of Experimental Medicine

\section{Member of the examination board}

Prof. Dr. Thomas Dresbach

Department of Anatomy and Embryology, University Medical Center Göttingen

Prof. Dr. Klaus-Armin Nave

Department of Neurogenetics, Max Planck Institute of Experimental Medicine

Prof. Dr. André Fischer

Department of Psychiatry and Psychotherapy, University Medical Center Göttingen

Date of oral examination: June $1^{\text {st }}, 2017$ 


\section{Acknowledgements}

First, I would like to thank Prof. Brose for the privilege of doing exciting research in his department and for the vast amounts of support I received from him during my PhD. I consider myself extremely lucky to be able to work for him.

I would like to thank Prof. Hannelore Ehrenreich and Dr. Camin Dean who, as the members of my $\mathrm{PhD}$ committee, gave me the courage to pursue new challenging directions and helped me to succeed. Thank you for your kindness and support.

I would not be able to concentrate on my research without the constant attention from Prof. Michael Hoerner and Ms. Sandra Drube, the coordinators of the Neuroscience Program. Apart from excellent logistical support, they made me feel welcomed far away from home during my whole time in Goettingen.

I am very grateful to all the members of Brose department for excellent working and partying environment. I am especially grateful to Liam Tuffy and Matthieu Hammer for all the help with the experiments, to Francisko Lopez Murcia for the help with patch clamp experiments, to Bekir Altas for moral support and encouragement, to Cordelia Imig and Ben Cooper who helped me on countless occasions, and for the rest of the great people I met during this period. I had nothing but good experience during my time in the lab.

Special thanks to Dr. Holger Taschenberger who taught me the patch clamp technique. Without his guidance I would not be able to perform some of the most important experiments during my $\mathrm{PhD}$.

Finally, I would like to thank the people who have been crucial for my research and beyond. I would like to thank Carolina Piletti Chatain for her hard and excellent work and for being so passionate about science. I extremely enjoyed being her supervisor and very grateful for the opportunity to continue working with her.

I cannot begin to express my gratitude to Dr. Dilja Krueger- Burg, my supervisor and mentor, who showed me the meaning of being a great scientist and at the same time, a great person. She was the best supervisor a $\mathrm{PhD}$ student can hope for, and I am extremely grateful for that.

Finally, I would like to mention my main collaborator inside and outside the lab, Hugo Cruces Solis. Without him, nothing would be possible. 
Dedicated to my parents,

my supervisor Dilja,

and to Hugo, who is the IgSF9b KO to my Nlgn2 KO 
“One can't understand everything at once, we can't begin with perfection all at once! In order to reach perfection one must begin by being ignorant of a great deal. And if we understand things too quickly, perhaps we shan't understand them thoroughly" - Fyodor Dostoyevsky, The Idiot

"Curioser and curioser!"

- Lewis Carroll, Alice's Adventures in Wonderland and Through the LookingGlass 


\section{Table of contents}

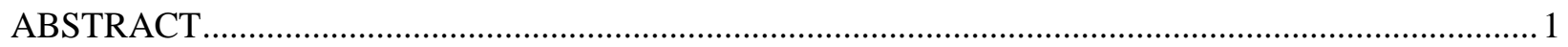

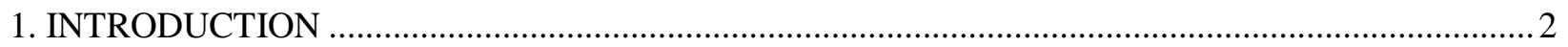

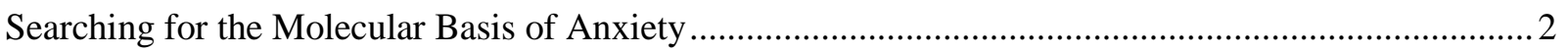

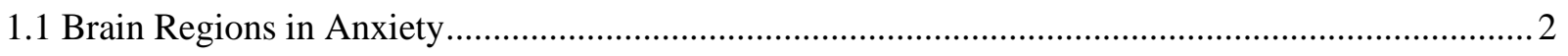

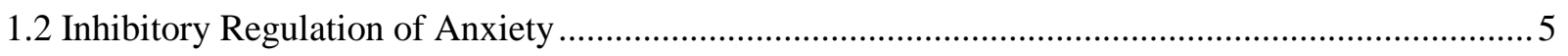

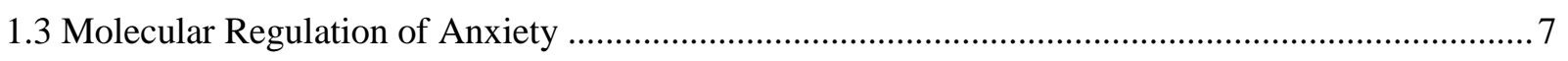

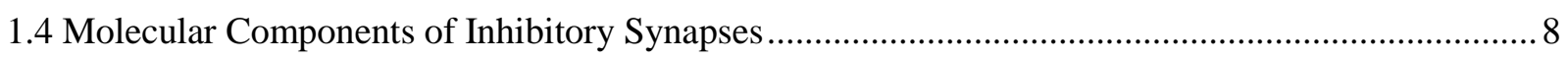

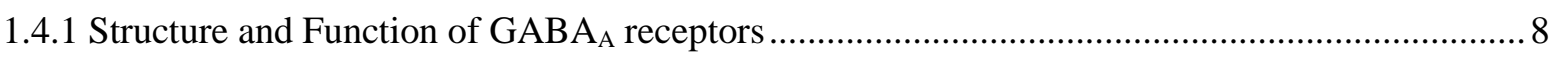

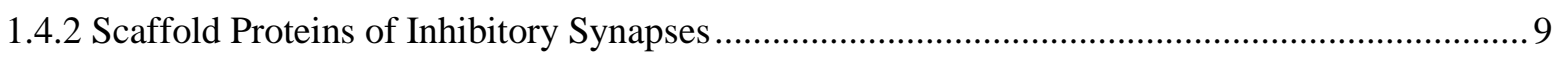

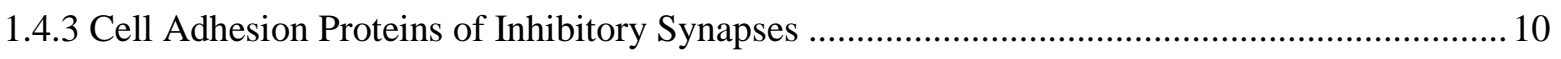

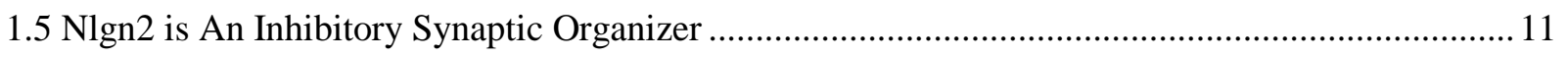

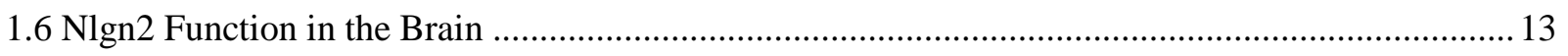

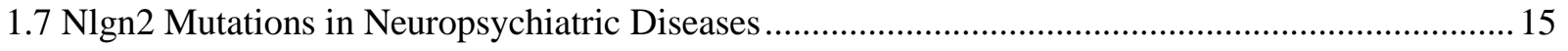

1.8 Nlgn2 in Maintenance of Excitation/Inhibition Ratio in Vivo..................................................... 15

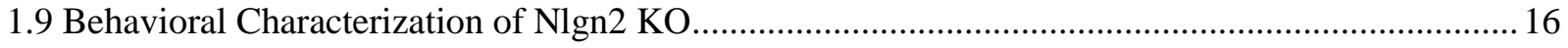

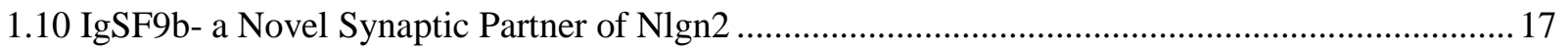

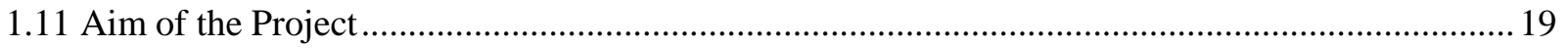

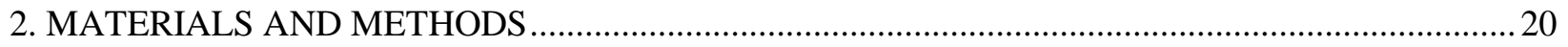

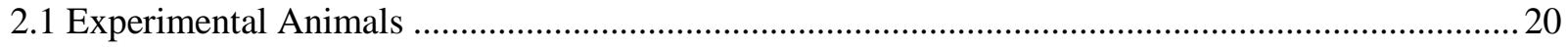

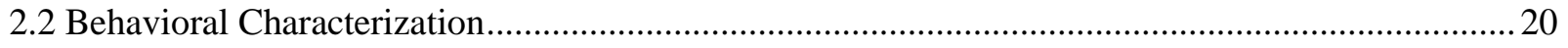

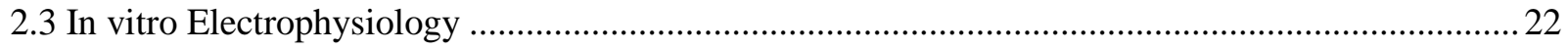

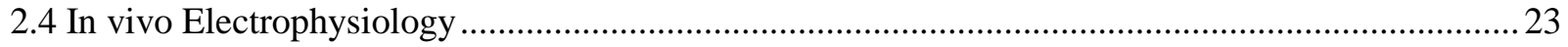

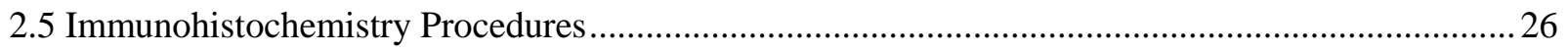

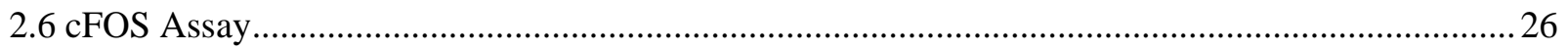

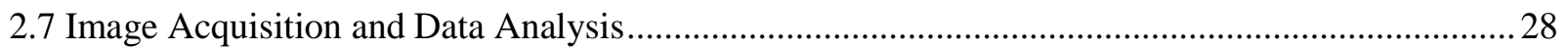

2.8 Retrograde Labeling of BA Neurons That Project to CeM............................................................... 32

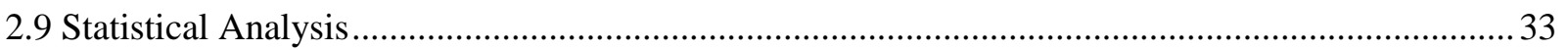

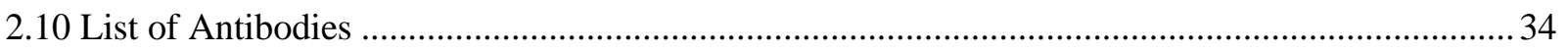

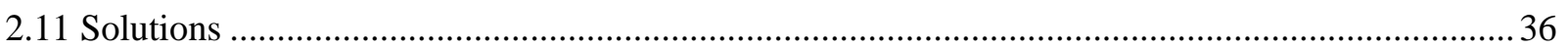

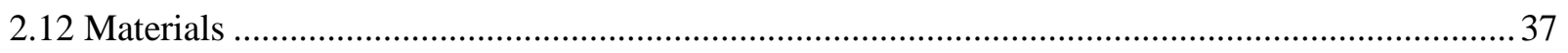

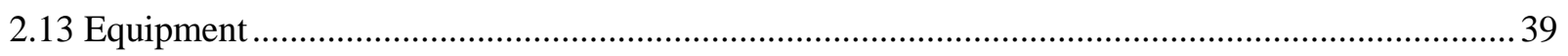

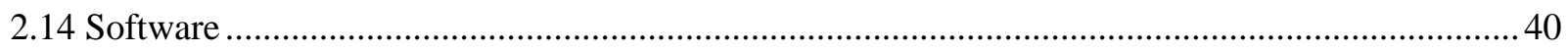


Chapter 1- Role of Neuroligin 2 in Inhibitory Synaptic Function and Anxiety- Associated Neuronal

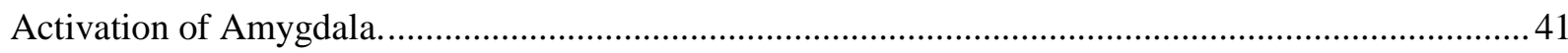

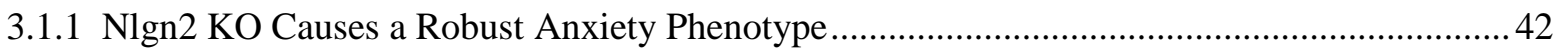

3.1.2 Nlgn2 KO Causes Reduced Locomotor Activity and Enhanced Freezing Specifically Under

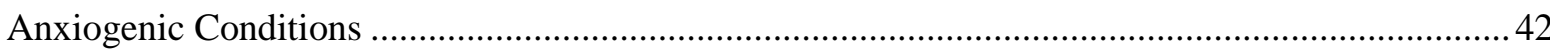

3.1.3 Enhanced cFOS Activation in Basal Amygdala Neurons of Nlgn2 KO Mice Upon Exposure to

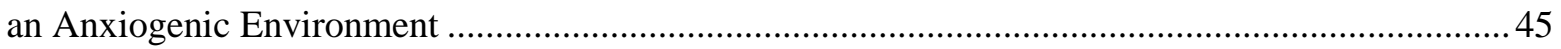

3.1.4 Nlgn2 is Localized to Inhibitory Synapses in the Basal Amygdala ...................................... 46

3.1.5 Nlgn2 KO Perturbs the Composition of Perisomatic Postsynaptic Sites in the Basal Amygdala 49

3.1.6 Nlgn2 KO Impairs Inhibitory Synaptic Transmission in the Basal Amygdala..... .51

3.1.7 Nlgn2 KO has Differential Effects on Anxiety-Associated Activation of Glutamatergic and

GABAergic Neurons in the Basal Amygdala .53

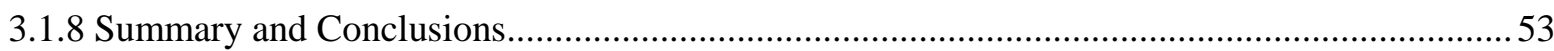

Chapter 2 - Role of IgSF9b in Regulation of Anxiety Phenotype of Nlgn2 KO.................................56

3.2.1 IgSF9b KO Rescues the Anxiety Phenotype of Nlgn2 KOs.... .57

3.2.2 IgSF9b KO Rescues the Anxiety Associated Overactivation of Centromedial, but not Basal, Amygdala in Nlgn2 KO Mice

3.2.3 IgSF9b KO Upregulates the Anxiety Associated Activation of Parvalbumin Neurons in Basal Amygdala.

3.2.4 IgSF9b KO Does Not Downregulate the Excitatory Output from Basal Amygdala to

Centromedial Amygdala in Nlgn2 KOs .63

3.2.5 Centromedial Amygdala Mediates Anxiety Related Processing In Vivo..... .65

3.2.6 IgSF9b KO Rescues the Enhanced Anxiety Associated Activation of CeM in Nlgn2 KOs......67

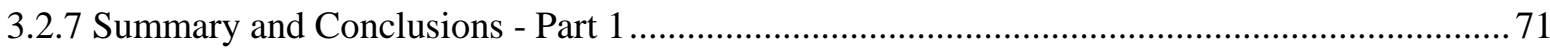

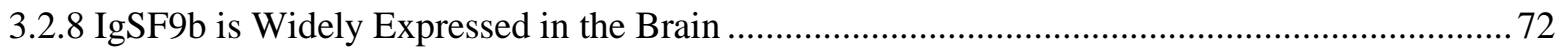

3.2.9 IgSF9b Does Not Colocalize with Synaptic Markers In Vivo ................................................ 72

3.2.10 IgSF9b is Apposed to Gamma 2 Subunit of GABA Receptor in Centromedial Amygdala .... 74

3.2.11 IgSF9b and Its Post Synaptic Partners are Expressed in Basal and Centromedial Amygdala.75

3.2.12 Nlgn2 KO and IgSF9b KO Reduce Perisomatic GABAr $\alpha 1$ Puncta in Brain Region- Specific

Manner .77

3.2.13 IgSF9b KO Does Not Rescue the Deficit in Gephyrin Puncta in Basal Amygdala of Double KOs 
3.2.14 Nlgn2 KO and IgSF9b KO Do Not Alter Gephyrin and S-SCAM Puncta in Centromedial

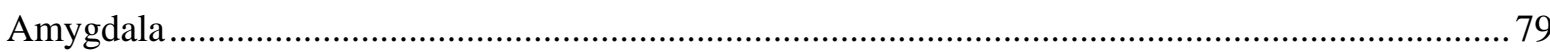

3.2.15 Nlgn2 KO and IgSF9b KO Perturb the Composition of Specifically Perisomatic Synapses .. 81

3.2.16 Nlgn2 KO and IgSF9b KO Do Not Alter the Overall Number of Inhibitory and Excitatory

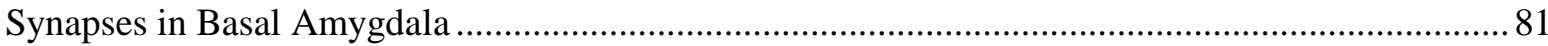

3.2.17 Nlgn2 KO Impairs Inhibitory Synaptic Transmission in Basal Amygdala ............................ 83

3.2.18 IgSF9b KO Enhances Inhibitory Synaptic Transmission in Centromedial Amygdala of Nlgn2

KOs 86

3.2.19 IgSF9b may be a novel regulator of inhibitory synapses onto inhibitory neurons.................. 86

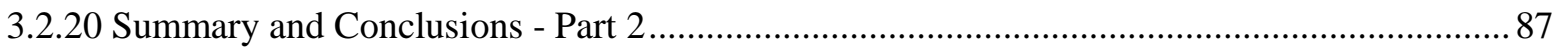

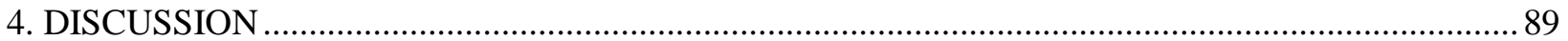

4.1 Consequences of Nlgn2 and IgSF9b Deletion on Anxiety- Related Behavior in Mice ...................89

4.1.1 Consequences of Nlgn2 Deletion on Anxiety- Related Behavior ........................................... 89

4.1.2 Consequences of Deletion of IgSF9b in Nlgn2 KO mice on Anxiety- Related Behavior ......... 90

4.2 Consequences of Nlgn2 Deletion on Synaptic Structure and Synaptic Function in the Anxiety-

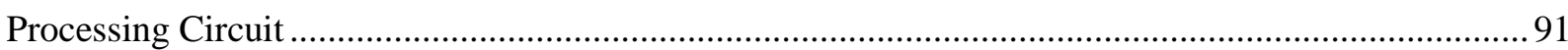

4.2.1 Effect of Nlgn2 Deletion on Inhibitory Synapses in Basal Amygdala ...................................91

4.2.2 Effect of Nlgn2 Deletion on Inhibitory Synapses in Centromedial Amygdala........................96

4.3 Consequences of IgSF9b Deletion on Synaptic Structure and Synaptic Function in Anxiety-

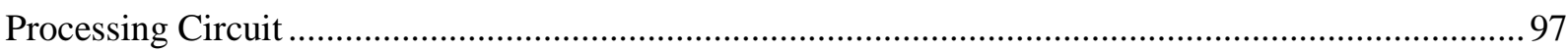

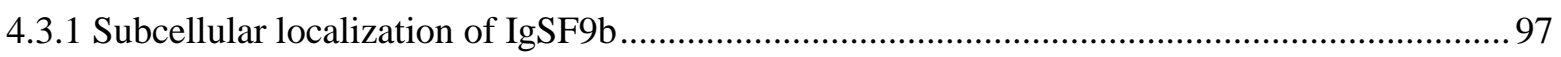

4.3.2 Consequences of IgSF9b deletion in basal amygdala ........................................................ 98

4.3.3 Consequences of IgSF9b Deletion in Centromedial Amygdala............................................. 98

4.4 Synaptic Structure and Synaptic Function in Anxiety- Processing Circuit in Double Nlgn2 IgSF9b

$\mathrm{KO}$ mice .99

4.5 Consequences of Nlgn2 and IgSF9b Deletion on Excitatory and Inhibitory Networks in Basal

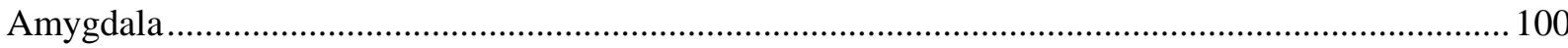

4.5.1 Consequences of Nlgn2 deletion on Neurons in BA........................................................... 100

4.5.2 Consequences of IgSF9b Deletion on Neurons in CeM ..................................................... 101

4.5.3 Combined Effect of Nlgn2 and IgSF9b Deletion on Neurons in BA.................................... 102

4.6 Consequences of Nlgn2 and IgSF9b Deletion on Anxiety- Associated Neural Activation of Basal

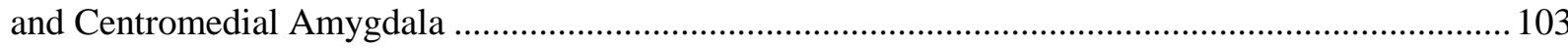

4.6.1 Anxiety- Associated cFOS Expression in Basal and Centromedial Amygdala ...................... 103

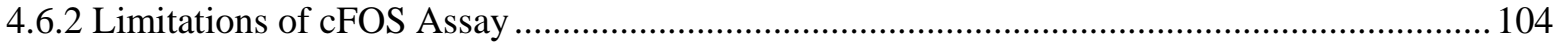




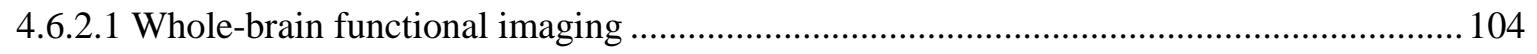

4.6.2.2. In vivo Recording of Neural Activity in Freely Behaving Mice................................... 105

4.6.3 Neural Activity of CeM During Exploration of the Open Field ............................................ 106

4.7 Conclusions: Implications for Molecular Mechanisms of Anxiety ............................................... 107

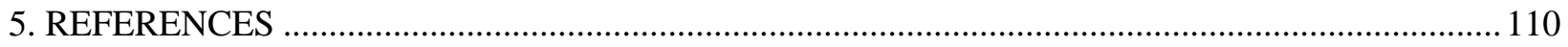




\section{List of abbreviations}

$\begin{array}{ll}\text { ASD } & \text { Autism spectrum disorders } \\ \text { BA } & \text { Basal amygdala } \\ \text { BLA } & \text { Basolateral amygdala } \\ \text { BNST } & \text { Bed nucleus of stria terminalis } \\ \text { CB } & \text { Calbindin } \\ \text { CCK } & \text { Cholecystokinin } \\ \text { CeA } & \text { Central amygdala } \\ \text { CeL } & \text { Centrolateral amygdala } \\ \text { CeM } & \text { Centromedial amygdala } \\ \text { EPM } & \text { Elevated plus maze }\end{array}$

GABA $\gamma$-Aminobutyric acid

Het Heterozygous

KO Knock out

LA Lateral amygdala

LDT Light dark box

mIPSC Mini inhibitory postsynaptic current

mPFC Medial prefrontal cortex

Nlgn Neuroligin

Nlgn2 Neuroligin 2

OFT Open field test

PV Parvalbumin

SOM Somatostatin

VGAT Vesicular GABA transporter

Vglut Vesicular glutamate transporter

vHPC Ventral hippocampus

WT Wild type 

ABSTRACT

\section{ABSTRACT}

Anxiety disorder is a debilitating condition that affects up to $10 \%$ of the adult population in the world. Mapping of brain activity in patients and in mouse models has shown that anxiety- related behavior is associated with abnormally high firing of basal amygdala and its downstream target nucleus, the centromedial amygdala. A plausible mechanism for this increased excitatory drive is dysfunction of amygdala inhibitory synapses, but the proteins that contribute to such dysfunction and that may serve as novel targets for pharmaceutical intervention are largely unknown. Here, I investigate the function of two synaptic adhesion proteins implicated in neuropsychiatric disorders, Neuroligin 2 (Nlgn2) and IgSF9b, at inhibitory synapses in the anxiety- processing circuitry in amygdala. Using a combination of behavioral assessment, quantitative morphological analysis, cFOS induction assay, anatomical tracing and in vivo and in vitro electrophysiology, I show that deletion of Nlgn2 causes a prominent anxiety phenotype and results in upregulation of activity of excitatory projection neurons in basal amygdala, which in turn robustly enhance the firing of centromedial amygdala under anxiogenic conditions. To identify additional inhibitory synaptic proteins involved in anxiety regulation, I investigated the function of IgSF9b, a novel cell adhesion molecule that interacts with Nlgn2 in vitro and hence potentially modulates the anxiety phenotype of Nlgn2 KO mice. I show that in sharp contrast to Nlgn2 KO mice, deletion of IgSF9b decreases anxiety behavior and enhances the activation of inhibitory interneurons in basal amygdala under anxiogenic conditions. Moreover, deletion of each protein affects distinct subsets of inhibitory synapses in basal and centromedial amygdala. Strikingly, those differential mechanisms interact in mice lacking both proteins to completely rescue anxiety phenotype by normalizing the anxietyassociated firing of centromedial amygdala. Specifically, deletion of IgSF9b rescues the enhancement of high gamma $(70-120 \mathrm{~Hz})$ band activity of centromedial amygdala in Nlgn2 $\mathrm{KO}$ mice during exposure to anxiogenic conditions. My findings indicate that Nlgn2 and IgSF9b play a crucial role in anxiety processing, thus providing important insights into the molecular mechanisms of anxiety and into potential drug targets for anxiety treatment. 


\section{INTRODUCTION}

\section{INTRODUCTION}

\section{Searching for the Molecular Basis of Anxiety}

Survival of an organism is crucially dependent on the ability of its brain to generate a precise behavioral response to threats. A complex neural network continuously categorizes multimodal environmental cues as safe or threating and, upon detecting a threating stimulus, commands the brainstem centers of motor and autonomic function to prepare the body for escape or fight. Dysfunction of this neural processing may give rise to excessive defensive behavior on the expense of other vital behaviors such as food seeking and mating, thus reducing the chances of survival.

In humans, avoidance of daily activities due to over apprehension of possible threats is the core symptom of anxiety disorder (LeDoux et al. 2017). Anxiety disorder is among the most common psychiatric disorders, with life time prevalence of $~ 30 \%$ in adults (Kessler et al. 2005). Studies of underlying mechanisms showed that anxiety patients have an enhanced activity of brain regions involved in processing of threat- related stimuli, which henceforth will be referred to as "anxietyrelated processing", and display abnormally low extracellular levels of the inhibitory neurotransmitter $\gamma$-Aminobutyric Acid (GABA) (Petty \& Sherman 1984; Etkin \& Wager 2007). Collectively, these findings indicate that decreased GABAergic neurotransmission in the anxiety processing brain regions is one of the key mechanisms of anxiety. Therefore, identifying the molecular factors that govern GABAergic neurotransmission across the anxiety processing circuitry is essential to understanding the molecular basis of anxiety.

\subsection{Brain Regions in Anxiety}

In the center of anxiety- related processing network lies the amygdaloid complex, a multinuclear structure located within medial temporal lobe. Amygdala nuclei regulate a wide range of behavioral responses to emotionally significant stimuli, such as food or threat. The deficits in emotional regulation following amygdala lesioning was demonstrated in monkeys, rodents, and, importantly, in humans, indicating that the function of amygdala is conserved across species (Weiskrantz 1956; Blanchard \& Blanchard 1972; Anderson \& Phelps 2001; Janak \& Tye 2015). 


\section{INTRODUCTION}

Investigation of the functional role of amygdala nuclei identified two nuclear complexes as key regions in the acquisition, storage and expression of conditioned fear: the basolateral complex and the central nucleus (LeDoux 2003, Figure 1.1). The basolateral complex (BLA) receives sensory information from thalamus, cortical association areas and prefrontal cortex through its lateral nucleus (LA), processes this information in the basal nucleus (BA) and sends it to the central nucleus (CeA). $\mathrm{CeA}$ (specifically centromedial nucleus $\mathrm{CeM}$ ) in turn projects to hypothalamus and brainstem to generate a behavioral output (Romanski et al. 1993; Sah et al. 2003). This serial processing of information (Figure 1.2) provides a useful platform to study the effect of genetic, pharmacological and functional manipulations of individual BLA nuclei on fear- related behavior.
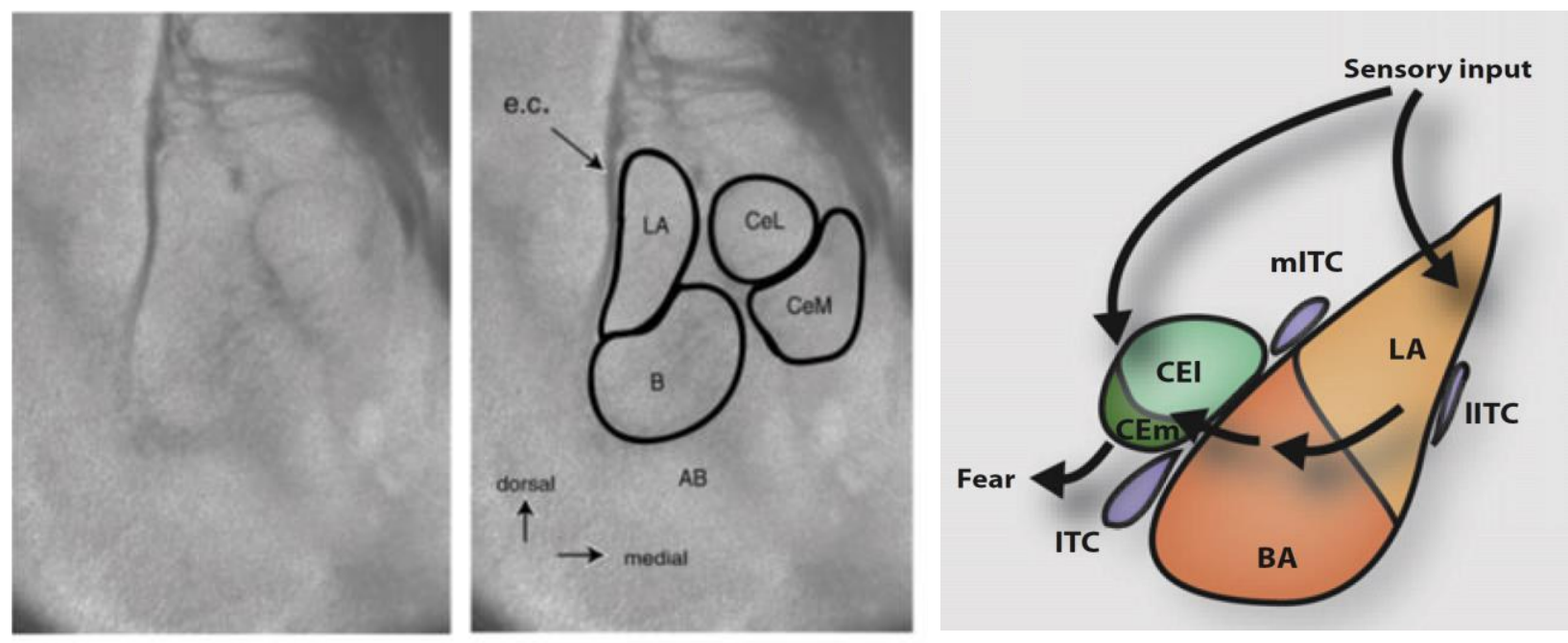

Figure 1.1| Serial processing of sensory information in BLA-CeA circuit.

Coronal section of BLA and CeA, with outlined lateral, basal, centrolateral and centromedial nuclei (left) and scheme of overall flow of sensory information across BLA- CeA circuit (right). CeM- centromedial nucleus. ITC- complex of GABAergic neurons that are involved in regulation of fear- related behavior. Adapted from Sah et al. 2003; Ehrlich et al. 2009.

Substantially less is known about the role of the BLA- CeA complex in the regulation of anxietyrelated behavior, although it is generally assumed that the circuity must at least partially overlap with the fear- processing circuitry (Tovote et al. 2015). Optogenetical stimulation of excitatory projections from BA to the lateral part of central nucleus $(\mathrm{CeL})$ in mice decreases the avoidance 


\section{INTRODUCTION}

of an exposed arena during an assay of anxiety (Tye et al. 2011). Moreover, activation of neurons projecting from the BA to the medial part of central nucleus (CeM) increases avoidance behavior in mice (Namburi et al. 2015). Since disrupted BA- CeM functional connectivity was found in patients with generalized anxiety disorder (Etkin et al. 2009), these findings support the key role of BLA projections to CeM in anxiety.

In addition to its intra-amygdalar interactions, BLA is part of a brain-wide anxiety-processing network (Figure 1.2). Medial prefrontal cortex (mPFC) sends a robust excitatory projection to BLA, which synapses onto inhibitory neurons and supresses BLA output to reduce fear expression in the absence of threat (Mcdonald et al. 1996; Sotres-Bayon et al. 2004; Likhtik et al. 2013). Additionally, BLA is reciprocally connected with ventral hippocampus (vHPC), and activation of BLA axon terminals was shown to excite hippocampal neurons and enhance avoidance behavior (Pikkarainen et al. 1999; Felix-Ortiz et al. 2013). The role of BLA long range connectivity in mediating anxiety- related behavior was recently demonstrated in vivo by showing that synchronized activity across the mPFC-vHPC- BLA circuit at specific frequency ranges encodes safe and danger zones during exploration of an open arena (Stujenske et al. 2014). The neural correlate of safety and danger encoding in CeM however remains unexplored.

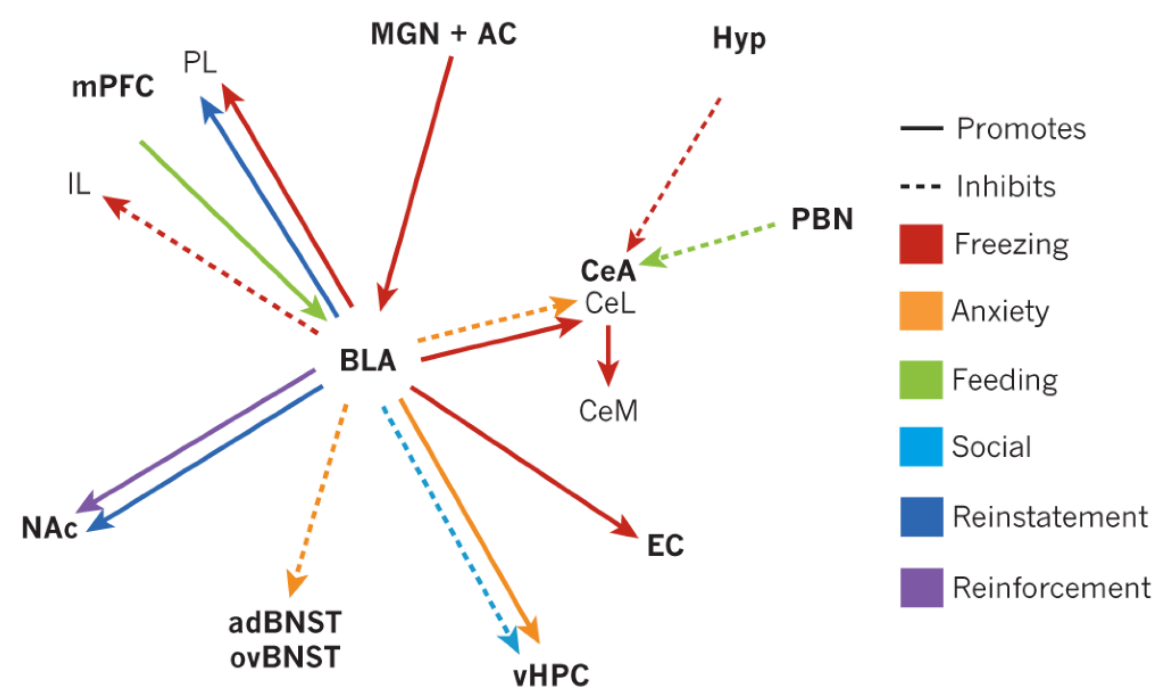

\section{Figure 1.2| BLA long-range connections in regulation of diverse behavioral domains.}

BLA regulates anxiety through excitation of vHPC and inhibition of Bed Nucleus of Stria Terminalis (BNST) and CeL. More functional BLA connections were described so far for fear- related behavior (here: 


\section{INTRODUCTION}

freezing, reinstatement and reinforcement) than for anxiety. AC, auditory cortex; adBNST, anterodorsal bed nucleus of the stria terminalis; EC, entorhinal cortex; Hyp, hypothalamus; IL, infralimbic cortex; MGN, medial geniculate nucleus; mPFC, medial prefrontal cortex; NAc, nucleus accumbens; ovBNST, oval nucleus of the BNST; PBN, parabrachial nucleus; PL, prelimbic cortex; vHPC, ventral hippocampus. Adapted from Janak \& Tye 2015.

\subsection{Inhibitory Regulation of Anxiety}

The excitatory output of BA is tightly regulated by GABAergic interneurons, which determine the spiking rate and coordinate the stimulus-specific activation of BA projection neurons. Interneurons comprise $20 \%$ of neuronal population in BA and form local inhibitory circuits that provide feed forward and feedback inhibition to BA projection neurons (Spampanato et al. 2011). There are at least 4 populations of interneurons, that can be distinguished by expression of calcium binding proteins, as well as by their postsynaptic targets: 1) Parvalbumin $^{+} / \mathrm{Calbindin}^{+}$; 2) Somatostatin $^{+} /$Calbindin $^{+}$; 3) $\quad \mathrm{VIP}^{+} /$Cholecystokinin $^{+} /$Calretinin $^{+}$; 4) Cholecystokinin ${ }^{+}$ (Kemppainen \& Pitkanen 2000; Muller et al. 2007; Muller et al. 2006; Spampanato et al. 2011; summarized in table 1.1). This anatomical heterogeneity underlies the functional heterogeneity of BA interneuron populations.

Table 1.1| Characteristics and function of interneurons in BA

$$
\text { Neuronal markers } \quad \text { Post synaptic targets Anxiety- related function* }
$$

\begin{tabular}{llll}
\hline General & Specific & & \\
\hline \hline Calbindin $(\mathrm{CB})$ & Parvalbumin (PV) & $\begin{array}{l}\text { Somata, axon initial } \\
\text { segment, proximal } \\
\text { dendrites }\end{array}$ & $\begin{array}{l}\text { Perisomatic inhibition of }{ }^{1} \\
\text { projectors to CeM }\end{array}$ \\
& Somatostatin (SOM) & Distall dendrites & $\begin{array}{l}\text { Dendritic disinhibition of }{ }^{1} \\
\text { projectors to CeM }\end{array}$ \\
& $\begin{array}{l}\text { Cholecystokynin } \\
(\text { CCK)** }\end{array}$ & $\begin{array}{l}\text { Somata, proximal } \\
\text { dendrites }\end{array}$ & $\begin{array}{l}\text { Perisomatic inhibition of } \\
\text { projectors to mPFC }\end{array}$ \\
\hline
\end{tabular}




\section{INTRODUCTION}

1. Wolff et al. 2014 2.Vogel et al. 2016b

* Contribution to expression of fear- related behavior

** Subpopulation of CCK neurons additionally expresses calretinin

The role of each class of inhibitory interneurons in regulation of the excitatory output of BA was studied mainly in the context of fear- related behavior using fear conditioning paradigm, during which an animal learns to associate neutral sensory stimulus with electric shock. Parvalbumin ${ }^{+}$ interneurons were shown to distinctly respond to "neutral" (auditory tone) or "negative" (foot shock) sensory stimuli, demonstrating that BA interneurons may encode the stimulus valence (neutral vs negative). During different stages of fear conditioning Parvalbumin ${ }^{+}$interneurons either directly inhibited or indirectly disinhibited principal neurons by inhibiting Somatostatin ${ }^{+}$ interneurons, enhancing the association between foot shock and auditory tone and consequently, the fear response (Wolff et al. 2014). This work identified that Parvalbumin ${ }^{+}-$Somatostatin $^{+}$projection neuron microcircuit in BA (Figure 1.3) is crucial for shaping a behavioral response to fear. Similarly, Cholecystokinin ${ }^{+}$interneurons form microcircuits with projection neurons targeting fear- regulating region in mPFC to modulate fear- related behavior (Vogel et al. 2016b). These studies establish the inhibitory network in BA as a regulator of stimulus- specific behavioral outputs in fear- related processing.

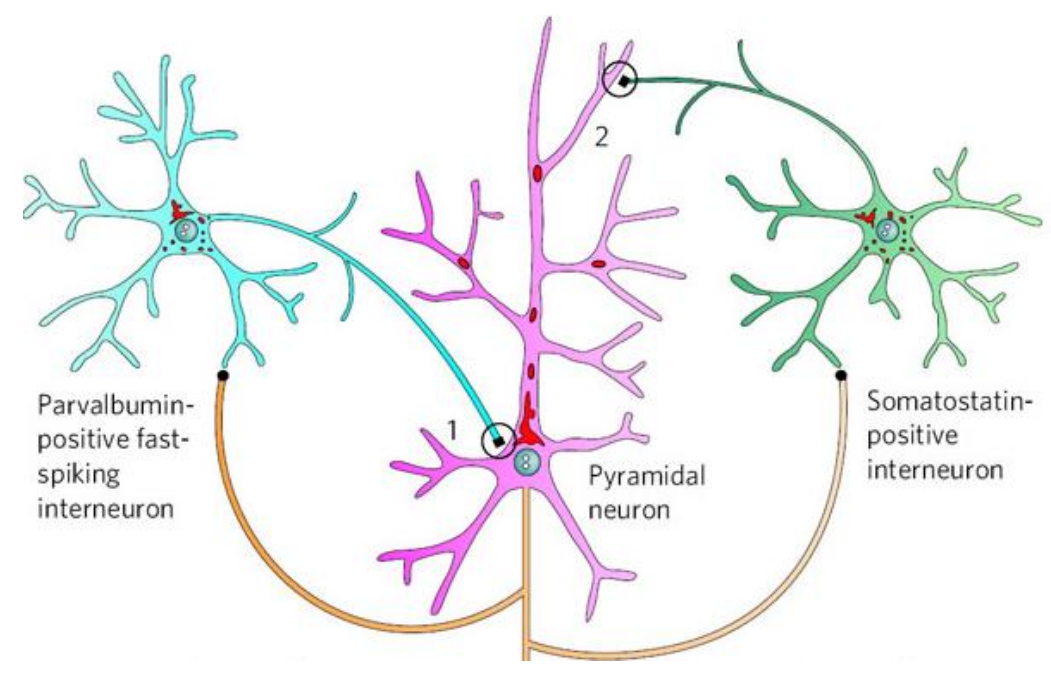

Figure 1.3| Microcircuit in BA that mediates fear- related behavior. Parvalbumin ${ }^{+}$interneurons form synapses on the soma and Somatostatin ${ }^{+}$interneurons form synapses on the dendrites of BA excitatory pyramidal- like neuron that projects to down stream regions. Adapted from Südhof 2008. 


\section{INTRODUCTION}

In stark contrast to cortical like- BA, CeM neurons have the same origin as striatal neurons and are predominantly GABAergic medium spiny projection neurons (Schiess et al. 1999). Very little is known about the expression of neuronal markers in CeM, which is under inhibitory control of CeL (specifically of CeL interneurons that express marker PKC $\delta$ ) but it is thought not to have local inhibitory circuits of its own (Ciocchi et al. 2010). Since CeM is a main region that translates BA inputs into fear response, a detailed analysis of both BA and CeM inhibitory regulation is essential for understanding the neural mechanisms underlying anxiety- related behavior.

\subsection{Molecular Regulation of Anxiety}

The function of inhibitory network in BA is mediated through GABA. BA has one of the highest concentrations of $\mathrm{GABA}_{\mathrm{A}}$ receptors in the brain, especially in the anterior part of BA (Niehoff \& Kuhar 1983). GABA A receptors mediate fast synaptic inhibitory transmission in BA (Sanders \& Shekhar 1995), and blocking their activity produces behavioral and autonomic manifestations of anxiety such as avoidance behavior, fight or flight response, increase in heart rate and blood pressure (Sanders \& Shekhar 1991; Sanders \& Shekhar 1995). The key role of GABA receptors in regulating anxiety- related processing in BA is further supported by widespread usage of benzodiazepines, pharmaceutical agents that enhance the affinity of GABA receptors to GABA, to alleviate anxiety in patients with anxiety related disorders (Smith 2001). It suggests that dysfunction of $\mathrm{BA} \mathrm{GABA}_{\mathrm{A}}$ receptors may be one of the major molecular mechanisms underlying decreased inhibitory tone in BA in anxiety disorders.

The contribution of chronic GABAergic dysfunction in BA to anxiety disorders is demonstrated in a mouse model of anxiety that lacks GABA synthetizing enzyme Glutamate Decarboxylase 65 (GAD 65). GAD $65 \mathrm{KO}$ mice show an increase in anxiety- related behaviours, that is accompanied by decreased inhibitory drive in some amygdala regions (Lange et al. 2014; Müller et al. 2015). Additionally, mice lacking Fmr1 protein have reduced synaptic GABA in BA. Mutations in Fmr1 are linked to Fragile X syndrome in humans, one of manifestations of which is anxiety (OlmosSerrano et al. 2010). These studies suggest that dysfunction of inhibitory synapses in BA may be a common pathophysiological pathway underlying anxiety in mouse models of neuropsychiatric disorders. Thus, understanding the molecular mechanisms that govern inhibitory synaptic function in BA may provide an insight into molecular mechanism underlying anxiety related disorders. 


\section{INTRODUCTION}

\subsection{Molecular Components of Inhibitory Synapses}

Fast synaptic inhibitory transmission in the central nervous system is mediated through signalling between highly specialized pre- and postsynaptic compartments that tightly regulate the frequency of action potentials produced by neurons and are therefore critical to all the aspects of brain function. Two major postsynaptic factors determine the efficiency of signalling at the inhibitory

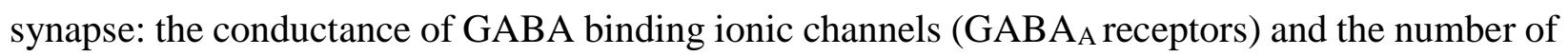
$\mathrm{GABA}_{\mathrm{A}}$ receptors at the synaptic sites. The functional properties of $\mathrm{GABA}_{\mathrm{A}}$ receptors are determined by their subunit composition, and their precise subcellular localization is achieved via coordinated action of scaffold proteins and adhesion proteins at postsynaptic sites. Loss of any part of this machinery has a severe impact on GABAergic function (Fritschy et al. 2012).

\subsubsection{Structure and Function of $\mathrm{GABA}_{\mathrm{A}}$ receptors}

$\mathrm{GABA}_{\mathrm{A}}$ receptors are transmembrane ionic channels which, upon binding of extracellular GABA, allow the hyperpolarization of the membrane via influx of chloride ions into the neuron. Each channel consists of a pentameric combination formed out of 15 subunits $\left(\alpha_{1-6}, \beta_{1-3}, \gamma_{1-3}, \delta, \rho_{1-2}\right)$, with the most common subunit composition consisting of two $\alpha$ subunits (generally $\alpha_{1}, \alpha_{2}$ or $\alpha_{3}$ ), two $\beta$ subunits and a $\gamma_{2}$ subunit (Macdonald \& Olsen 1994; Whiting et al. 1999). $\mathrm{GABA}_{\mathrm{A}}$ receptors show high molecular and functional heterogeneity across brain regions and cellular compartments (Fritschy \& Mohler 1995), but the role of individual GABA subunits in receptor function remains largely unknown.

A detailed study of expression and function of different GABA receptor subunits in BA and CeA revealed that $\alpha 1, \alpha 2$ and $\alpha 3$ subunits are highly expressed in BA. Interestingly, $\alpha 1$ subunit is absent and $\alpha 3$ subunit is expressed at a low level in CeA, suggesting that inhibitory transmission in $\mathrm{BA}$ and $\mathrm{CeA}$ is mediated through different sub types of $\mathrm{GABA}_{\mathrm{A}}$ receptors. Activation of both $\alpha 1$ and $\alpha 2$ subunits, and to a lesser extent $\alpha 3$ subunit of $\mathrm{GABA}_{\mathrm{A}}$ receptors increases inhibitory synaptic input on pyramidal neurons in BA (Marowsky et al. 2004). No postsynaptic inhibitory currents mediated by other types of inhibitory receptors, such as glycine, GABA $A_{B}$ and GABAC receptors were detected in basal amygdala (Sanders \& Shekhar 1995; Marowsky et al. 2004). 


\section{INTRODUCTION}

Taken together, these studies indicate that inhibitory transmission within basal amygdala circuits is predominantly mediated by $\mathrm{GABA}_{\mathrm{A}}$ receptors that contain $\alpha 1$ and $\alpha 2$ subunits.

Several studies have investigated the physiological function of different $\mathrm{GABA}_{\mathrm{A}}$ receptor subunits using a $\mathrm{KO}$ approach of individual subunits in vivo. These studies demonstrated that GABAry2 KO mice die shortly after birth due to severe reduction in channel conductance and reduced postsynaptic clustering of $\mathrm{GABA}_{\mathrm{A}}$ receptors, revealing the pivotal role of $\gamma_{2}$ subunit in GABAergic transmission (Günther et al. 1995; Essrich et al. 1998). In contrast, deletion of $\alpha_{1}$ subunit is not lethal and does not lead to severe behavioral phenotypes, although it causes loss of $\sim 60 \%$ percent of $\mathrm{GABA}_{\mathrm{A}}$ receptors in cerebral cortex (Kralic et al. 2002), suggesting that $\alpha_{1}$ may be at least partially functionally redundant. These and additional studies emphasized that the mechanisms that determine the composition of $\mathrm{GABA}_{\mathrm{A}}$ receptors are crucial for inhibitory function. These mechanisms most likely involve interactions with scaffold and adhesion proteins that may bind specific $\mathrm{GABA}_{\mathrm{A}}$ receptor subunits at the post synapse (Fritschy et al. 2012).

\subsubsection{Scaffold Proteins of Inhibitory Synapses}

Scaffold proteins are cytosolic proteins that attach to the cytoskeleton in proximity of post synaptic membrane and form multimeric complexes that serve as aggregating platforms for various components of post synaptic density (Sheng 1996). The major scaffold protein of inhibitory synapses is gephyrin. Gephyrin forms hexagonal lattice underneath the postsynaptic membrane and aggregates $\mathrm{GABA}_{\mathrm{A}}$ and glycine receptors upon their insertion into the postsynaptic membrane via multiple binding sites. During synapse formation, gephyrin is recruited to the postsynaptic membrane via interaction with the GDP/GTP exchange factor collybistin, which anchors gephyrin to the membrane by binding both gephyrin and membrane lipids ( Betz et al. 2000; Poulopoulos et al. 2009). Apart from driving the clustering of synaptic GABA receptors, gephyrin binds the synaptic adhesion protein Nlgn2, thus indirectly contributing to stabilization of the synaptic structure (see below). The crucial role of gephyrin in synaptic transmission is demonstrated by the lethality of gephyrin KO mice. (Feng et al. 1998) and by dramatic reduction of synaptic GABA receptors and the amplitudes of postsynaptic inhibitory currents in gephyrin KO neurons (Kneussel et al. 1999). Importantly, gephyrin is required for synaptic clustering of some, but not all subtypes 


\section{INTRODUCTION}

of GABA receptors (Kneussel, Brandstatter, et al. 2001), pointing out to existence of additional factors that modulate the receptor content of the synapse.

S-SCAM is an additional scaffold protein that, unlike gephyrin, is expressed both at excitatory and inhibitory synapses. At inhibitory synapses, it binds several proteins including core inhibitory synaptic protein Neuroligin 2 (see below) and notably, an adhesive dystrophin glycoprotein complex that was proposed to mediate multiple synaptic functions such as transsynaptic signal transduction, cytoskeleton rearrangement and focal adhesion (Lévi et al. 2002). S-SCAM therefore may expose the core components of inhibitory synapse to modulation by extrasynaptic signals, adding another level of complexity to functional regulation of inhibitory synapses.

\subsubsection{Cell Adhesion Proteins of Inhibitory Synapses}

Postsynaptic targeting of GABA receptors to precisely oppose presynaptic vesicle release sites is essential for neural signalling and requires coordinated organization of pre- and postsynaptic compartments during synaptic maturation. Cell adhesion proteins such as the presynaptic Neurexins and the postsynaptic Neuroligins (Nlgns) stabilize the synapse following synaptic assembly. During early stages of synaptic maturation, Neurexins and Nlgns interact transsynaptically to drive clustering of core components of pre and postsynaptic sites, respectively (Craig \& Kang 2007; Krueger et al. 2012). Specifically, establishment of initial synaptic contact triggers postsynaptic clustering of Nlgns, which in turn recruits scaffold proteins and receptors to the postsynaptic sites. Simultaneously, clustering of Neurexins is important for organizing the presynaptic vesicle release machinery (Dean et al.; 2003; Graf et al. 2004; Dean \& Dresbach 2006). Nlgn- Neurexin interactions are therefore crucial for equipping the nascent synapse with core proteins that mediate synaptic signalling.

Following synaptic specialization, Nlgns and Neurexins stay attached to each other and physically reinforce the synaptic contact as long as the synapse is active (Chubykin et al. 2005). Apart from synaptic stabilization, Nlgns stabilize the postsynaptic density by binding scaffold proteins and receptors at the post synaptic sites (Krueger et al. 2012). Additionally, intracellular Nlgn 


\section{INTRODUCTION}

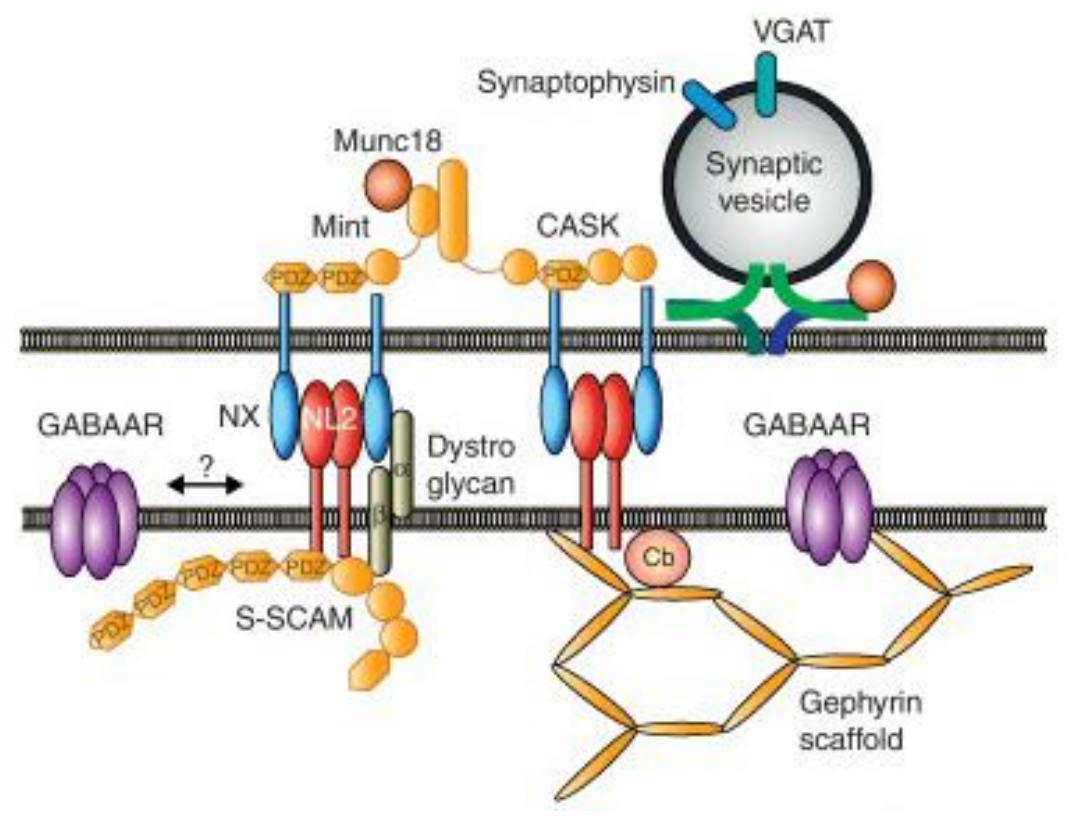

Figure 1.4| Synaptic interactions of Nlgn2 at inhibitory synapses. Adapted from Krueger et al. 2012.

interactions likely promote the clustering of receptors in an activity dependent manner, thus plastically enhancing the synaptic function in highly active networks (Hu et al. 2015). While the precise molecular mechanisms of their function are unknown, it is evident that Nlgns are essential for structural and functional aspects of the synapse.

The Nlgn family is encoded by five genes in humans NLGN1, NLGN2, NLGN3 NLGN4 and NLGN4Y, with NLGN4Y being absent from rodents (Ichtchenko et al. 1996). Nlgn1 and Nlgn2 act exclusively at excitatory and inhibitory synapses, respectively, while Nlgn3 is localized to both types and Nlgn4 is localized to inhibitory glycinergic synapses in retina and brain stem, and hippocampus (Varoqueaux et al. 2006; Budreck \& Scheiffele 2007; Hoon et al. 2011; Hammer et al. 2015). This differential expression allows each Nlgn to specify the functional properties of distinct types of synapses, such that Nlgn1 regulates the maturation and function of excitatory synapses and Nlgn2 of inhibitory synapses.

\subsection{Nlgn2 is An Inhibitory Synaptic Organizer}




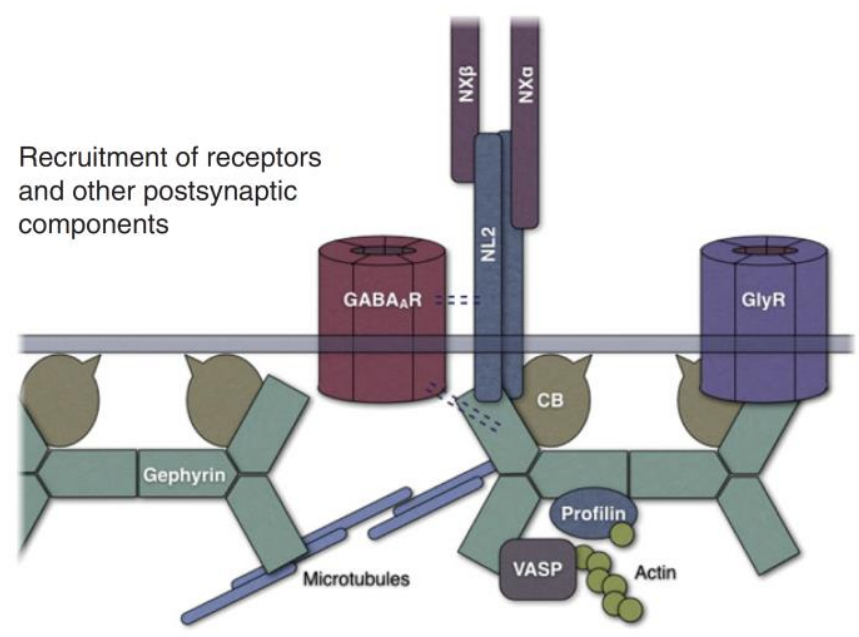

Figure 1.5| Nlgn2- Gephyrin- GABAA receptor complex.

Nlgn2 tethers gephyrin to post synaptic membrane and together Nlgn2 and gephyrin recruit GABA receptors to the inhibitory synapses. CB- collybistin. Adapted from Poulopoulos et al. 2009.

Nlgn2 is the first known adhesion protein constitutively present at inhibitory postsynaptic sites, where it assembles the synaptic components during synaptic maturation and maintains synaptic function of mature synapses via its multiple intracellular and extracellular interactions (Varoqueaux et al. 2006; Poulopoulos et al. 2009). Nlgn2 structurally and functionally couples pre and postsynaptic sites via interaction with Neurexin and mediates inhibitory specification and function via interactions with scaffold proteins and $\mathrm{GABA}_{\mathrm{A}}$ receptors (Poulopoulos et al. 2009; Nguyen et al. 2016, Figure 1.4).

The most well-characterized postsynaptic function of Nlgn2 is activation of the gephyrincollybistin complex. Nlgn2 binds gephyrin through a fifteen amino acid stretch at its cytoplasmic tail and simultaneously activates collybistin, which in turn targets gephyrin to the synaptic membrane (see above) (Poulopoulos et al. 2009, Figure 1.5). The amount of Nlgn2- gephyrin complexes at postsynaptic sites determines the amount of functional $\mathrm{GABA}_{\mathrm{A}}$ receptors and the amplitudes of post synaptic inhibitory currents (Poulopoulos et al. 2009; Antonelli et al. 2014), demonstrating the central role of Nlgn2 in assembly of the post synaptic machinery.

Nlgn2 may influence GABAergic transmission by directly binding to specific subunits of GABA receptors and inducing their clustering independently of gephyrin (Dong et al. 2007). Supporting 


\section{INTRODUCTION}

this notion, Nlgn2 was shown to promote the incorporation of GABAr $\alpha_{1}$ subunit into functional GABA receptors, leading to alteration in kinetics of inhibitory currents (Fu \& Vicini 2009). These findings suggest that Nlgn2 shapes inhibitory synaptic function not only by determining the number of GABA receptors, but also by specifying their functional properties.

In addition to interaction with gephyrin- collybistin complex and GABA receptors, Nlgn2 binds to $\beta$-dystroglycan, an S-SCAM and actin binding protein (Sumita et al. 2007; Woo et al. 2013). While the functional importance of these interactions was not experimentally addressed so far, it was proposed that S-SCAM may stabilize the synaptic localization of Nlgn2 and may mediate a cross talk between Nlgn2 and extrasynaptic proteins (Fritschy et al. 2012; Woo et al. 2013). In summary, the wealth of synaptic interactions enables Nlgn2 to organize and regulate inhibitory synaptic transmission throughout all stages of life of the synapse.

\subsection{Nlgn2 Function in the Brain}

Studies in vivo indicate that Nlgn2 stabilizes the synaptic structure once the initial synaptic contact is formed, hence determining the number of functional inhibitory synapses in each neuron (Chubykin et al. 2007; Jedlicka et al. 2011). Consequently, Nlgn2 maintains synaptic transmission at the inhibitory synapses in cortex, hippocampus, midbrain, cerebellum and retina (summarized at table 1.2; Gibson et al. 2009; Poulopoulos et al. 2009; Hoon et al. 2009; Jedlicka et al. 2011; Zhang et al. 2015). All of these studies found that Nlgn2 is not involved in any aspect of maturation and function of excitatory synapses, validating the role of Nlgn2 as organizer of exclusively inhibitory synaptic function.

Does Nlgn2 acts at all inhibitory synapses in the brain? The answer, based on cell region- specific quantification of GABA receptors, analysis of kinetics of inhibitory currents and modelling of inhibitory transmission in Nlgn2 KOs, appears to be negative. According to these studies, Nlgn2 regulates the function of specifically perisomatic inhibitory synapses (Poulopoulos et al. 2009; Jedlicka et al. 2011), which points to either a cell region- specific function and/or, as most of perisomatic synapses in cortical structures are formed by Parvalbumin positive neurons, a cell type- specific function. Consistent with both notions, Nlgn2 does not regulate the function of 


\section{INTRODUCTION}

inhibitory terminals formed by Somatostatin positive neurons, which mainly contact dendrites (Gibson et al. 2009). Therefore, Nlgn2 regulates the function of subpopulation of inhibitory synapses.

The functional specificity of Nlgn2 cannot be explained by its differential expression at cellular compartments, as Nlgn2 is expressed in the majority of inhibitory synapses, including dendritic synapses (Varoqueaux et al. 2004). Therefore, there must be synaptic factors that limit the function of Nlgn2 to specific subpopulation of synapses. Given that Nlgn2 regulates differential aspects of inhibitory transmission in distinct brain regions (Table 1.2), these factors must fine- tune Nlgn2 function in brain region- specific manner.

Table 1.2| Summary of electrophysiological characterization of Nlgn2 KO.

\begin{tabular}{|c|c|c|c|c|c|}
\hline & mIPSC & & sIPSC & & eIPSC \\
\hline Brain region & Frequency & Amplitude & Frequency & Amplitude & Amplitude \\
\hline $\begin{array}{l}\text { Cerebellum } \\
\text { (Purkinje } \\
\text { cells)*1 }\end{array}$ & $\sim 70 \%$ decrease & $\leftrightarrow$ & & & $\begin{array}{l}\sim 50 \% \\
\text { decrease }\end{array}$ \\
\hline Medulla $^{2}$ & $\sim 70 \%$ decrease & $\sim 25 \%$ decrease & $\sim 70 \%$ decrease & $\sim 50 \%$ decrease & $\begin{array}{l}\sim 50 \% \\
\text { decrease }\end{array}$ \\
\hline $\begin{array}{l}\text { Hippocampus } \\
(\mathrm{CA} 1)^{2}\end{array}$ & $\sim 50 \%$ decrease & $\leftrightarrow$ & $\sim 50 \%$ decrease & $\leftrightarrow$ & \\
\hline $\begin{array}{l}\text { Hippocampus } \\
\text { (dentate } \\
\text { gyrus) }\end{array}$ & $\leftrightarrow$ & $\sim 25 \%$ decrease & & & \\
\hline $\begin{array}{l}\text { Somatosensory } \\
\text { cortex }\end{array}$ & $\leftrightarrow$ & $\sim 25 \%$ decrease & & & $\begin{array}{l}\sim 50 \% \\
\text { decrease }\end{array}$ \\
\hline
\end{tabular}

* Conditional KO restricted to cerebellum.

SIPSC- spontaneous inhibitory postsynaptic currents, eIPSC- evoked inhibitory postsynaptic currents.

1. Zhang et al. 2015 2. Poulopoulos et al. 2009 3. Jedlicka et al. 2011 4. Gibson et al.2009 


\section{INTRODUCTION}

To summarize, Nlgn2 critically functions to maintain inhibitory synaptic transmission at perisomatic synapses. The precise role of Nlgn2 in individual aspects of neural transmission (e.g vesicle release, number of active synapses, composition of receptors), as well as mechanisms that define synapse- specific Nlgn2 function, are currently unknown.

\subsection{Nlgn2 Mutations in Neuropsychiatric Diseases}

The prominent role of Nlgn2 in organizing the inhibitory synapse positions Nlgn2 as a potential contributor to cognitive diseases associated with altered synaptic function, such as autism spectrum disorder (ASD) and schizophrenia (Thomas C. Südhof 2008; Harrison \& Weinberger 2005; Rubenstein \& Merzenich 2003). Supporting the involvement of Nlgns in neuropathophysiological conditions, multiple mutations in Nlgn3 and Nlgn4 were identified in patients with ASD (Zoghbi $\&$ Bear 2012). Moreover, six missense mutations in Nlgn2 were identified in a cohort of patients with schizophrenia, including a loss-of-function mutation that decreased GABAergic synaptic function (Sun et al. 2011). Additionally, one study found a duplication in gene region that includes Nlgn2 gene in patient with mental retardation (Belligni et al. 2012), although the contribution of other genes from the duplicated region to the phenotype was not ruled out.

Recently, a de-novo heterozygous nonsense mutation in Nlgn2 was reported in a patient with severe anxiety, obsessive- compulsive behaviors, autism, short attention span and global developmental delays (Parente et al 2016). No other clinically- relevant mutations were present in this patient, suggesting that the phenotype was caused by the Nlgn2 mutation. Anxiety and schizophrenia are co- morbid conditions (Braga et al. 2013), and pathophysiology of both is strongly linked to deficits in inhibitory transmission (Rubenstein \& Merzenich 2003; Lewis et al. 2005), which may explain how Nlgn2 mutations contribute to two distinct neuropsychiatric conditions. However, the exact neural substrate affected by mutations of Nlgn2 in vivo is not fully described.

\subsection{Nlgn2 in Maintenance of Excitation/Inhibition Ratio in Vivo}

Given that Nlgn2 specifically regulates inhibitory transmission, it was proposed that Nlgn2 mutations may lead to pathophysiological conditions by altering the ratio of excitation to inhibition 


\section{INTRODUCTION}

in brain regions implicated in autism and schizophrenia, such as prefrontal cortex and hippocampus (Amaral et al. 2008; Harrison \& Weinberger 2005). This hypothesis was tested in several studies that manipulated Nlgn2 levels or disrupted its function in vivo. Overexpression of Nlgn2 increased synaptic inhibitory transmission in prefrontal cortex and led to deficits in social interactions. Additionally, enhancement of Nlgn2 expression and pharmacological dissociation of Nlgn2 from Neurexin1 in hippocampus of adult mice decreased and increased social aggression, respectively, as well as increased anxiety (Hines et al. 2008; Kohl et al. 2013; van der Kooij et al. 2014). Finally, conditional deletion of Nlgn2 in medial prefrontal cortex of adult mice increased the excitation/inhibition ratio of pyramidal neurons, which was accompanied by decreased anxiety and fear conditioning deficits, but not by prominent social behavioral deficits (Liang et al. 2015). Interestingly, pharmacological disruption of Nlgn2 extracellular function in prelimbic cortex, one of the regions of medial prefrontal cortex, enhanced certain aspects of fear- related learning (Ye et al. 2016). These studies show that Nlgn 2 continuously maintains inhibitory transmission at mature synapses and that Nlgn2 is critically involved in regulation of clinically- relevant phenotypes, and thus may be a novel pharmaceutical target. However, a variety of phenotypes reported in these studies strongly suggest that Nlgn2 function in vivo is highly dependent on a brain region, limiting the applicability of region-specific conditional KO to human patients with global Nlgn mutations.

\subsection{Behavioral Characterization of Nlgn2 KO}

In light of attention that loss-of-function mutations in Nlgn2 received due to their link to ASD, it is surprising that only two studies so far aimed to understand the contribution of constitutive Nlgn2 KO to autism-related phenotypes. These studies reported that social interactions, measured as mouse interaction with mouse vs object and novel mouse vs familiar mouse, are not reduced in Nlgn2 KO, demonstrating that Nlgn2 is not involved in regulation of one of the core symptoms of ASD (Wöhr et al. 2014; Blundell et al. 2009). One study found a prominent reduction in locomotor activity in Nlgn2 KO (Wöhr et al. 2014), which was detected while mice explored a novel environment, and hence may be a product of novelty- induced freezing rather than locomotor impairment. The results of all the behavioral tests performed on Nlgn2 KO are summarized in table 1.3. 


\section{INTRODUCTION}

Table 1.3| Summary of behavioral deficits in Nlgn2 in comparison to WTs.

$\begin{array}{lll}\text { Anxiety } & \begin{array}{l}\text { Locomotor } \\ \text { activity }^{2}\end{array} & \begin{array}{l}\text { Sensory and } \\ \text { motor } \\ \text { function }^{1,2}\end{array}\end{array} \quad \begin{aligned} & \begin{array}{l}\text { Social } \\ \text { behavior }^{2}\end{array} \\ & \end{aligned}$

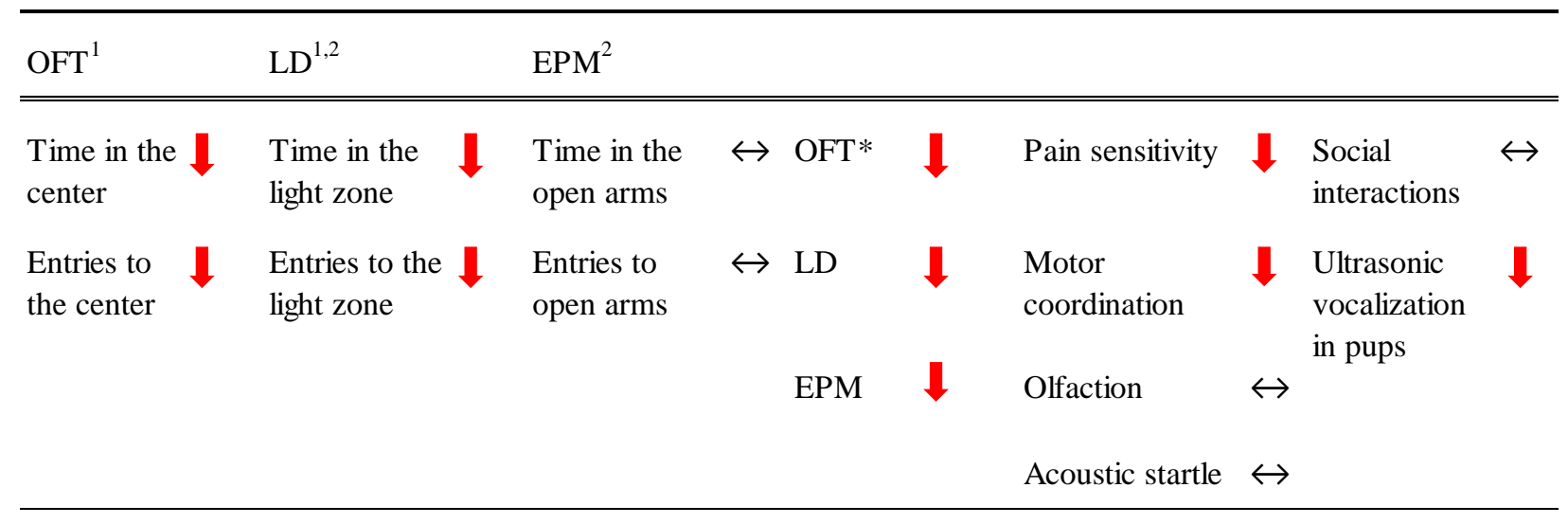

* Blundell et al. reported that locomotor activity is not altered in Nlgn2 KOs.

1. Blundell et al. 2009 2. Wöhr et al. 2014

As loss-of-function mutation of Nlgn2 was recently identified in a patient with severe anxiety (Parente et al. 2016), it is particularly important that a prominent anxiety related phenotype was reported in Nlgn2 KO mice (Blundell et al. 2009). This indicates that Nlgn2 must play a central role in the neural circuitry underlying anxiety behaviors, but to date, virtually nothing is known about the function of Nlgn2 in these circuits.

\subsection{IgSF9b- a Novel Synaptic Partner of Nlgn2}

The known synaptic partners of Nlgn2 including Neurexins, gephyrin, collybistin and GABA receptors majorly contribute to maintenance of inhibitory synaptic transmission, highlighting the central role of Nlgn2 at the inhibitory synapse. Recently, the adhesion protein IgSF9b was shown to interact with Nlgn2 through common binding to scaffold protein S-SCAM (Figure 1.6) and to regulate the number of inhibitory contacts onto inhibitory interneurons (Woo et al. 2013). These features indicate that IgSF9b may be a novel inhibitory synaptic regulator that modulates the function of Nlgn2. 


\section{INTRODUCTION}

IgSF9b belongs to a superfamily of IG adhesion proteins reported to regulate dendritic arborisation and synaptic maturation (Shi et al. 2004). IgSF9b is a transmembrane protein that contains five Ig domains, two fibronectin domains, one transmembrane domain and a C-terminal with PDZ binding motive. IgSF9b was recently identified as a novel inhibitory synaptic adhesion protein that is selectively present at inhibitory synapses. While precise role of IgSF9b in inhibitory circuits was not studied in vivo, post synaptic knockdown of IgSF9b in hippocampal cell cultures reduced the number of inhibitory synapses and decreased the frequency of mIPSCs, indicating that IgSF9b may regulate inhibitory synaptic development and/or maturation. Although the subcellular expression pattern and the function of IgSF9b closely resemble those of Nlgn2, there are three important distinctions. First, IgSF9b can mediate homophilic adhesion, indicating that IgSF9b function may not be limited to post synaptic sites. Second, IgSF9b is expressed at the periphery, and not at the core, of inhibitory synapses. Finally, IgSF9b predominantly regulates inhibitory transmission of synapses formed onto inhibitory neurons (Woo et al. 2013). Taken together, this suggests that IgSF9b and Nlgn2 may regulate inhibitory function by distinct mechanisms. Considering that both IgSF9b and Nlgn2 interact with S-SCAM, IgSF9b may modulate the synaptic function of Nlgn2 in specific populations of inhibitory synapses.

IgSF9b was linked to major depressive disorder, but its physiological function in vivo was not studied (Shyn et al. 2011). As major depressive disorder shows high co-morbidity with anxiety (Kendler et al. 1992), and in light of possible interaction of IgSF9b with anxiety- related Nlgn2, investigating the interaction of both IgSF9b and Nlgn2 in regulation of inhibitory transmission in anxiety processing circuit may lead to important insights into molecular mechanisms of anxiety.

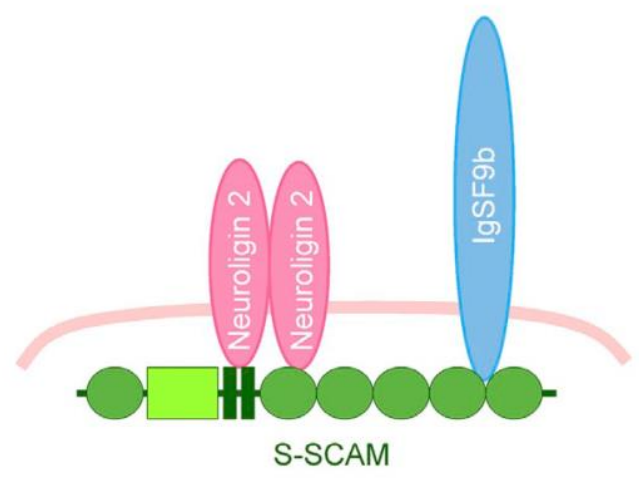

Figure 1.6 Interaction of IgSF9b and Nlgn2 at the post synaptic membrane. Adapted from Woo et al. 2013. 


\section{INTRODUCTION}

\subsection{Aim of the Project}

This project aims to characterize the molecular mechanisms underlying anxiety- related disorders by investigating the function of two synaptic adhesion proteins, NIgn2 and IgSF9b, in the anxiety processing circuit. Specifically, I address two questions:

1. What is the circuit, cellular and synaptic mechanism underlying anxiety- like phenotype of Nlgn2 KO mice?

2. Does IgSF9b deletion modulate the anxiety- like phenotype of Nlgn2 KO mice and if yes, what is the circuit, cellular or synaptic mechanism underlying this modulation?

To address these questions, I use a top-bottom approach (Figure 1.7) first identifying the brain regions, then identifying the type of neurons (excitatory vs inhibitory) and finally the synaptic proteins which function is altered in Nlgn2 KO and/or IgSF9b KO mice. My work describes, for the first time, the function of synaptic adhesion proteins in the inhibitory circuitry of amygdala and their profound impact on anxiety- related processing in vivo and anxiety- related behavior.

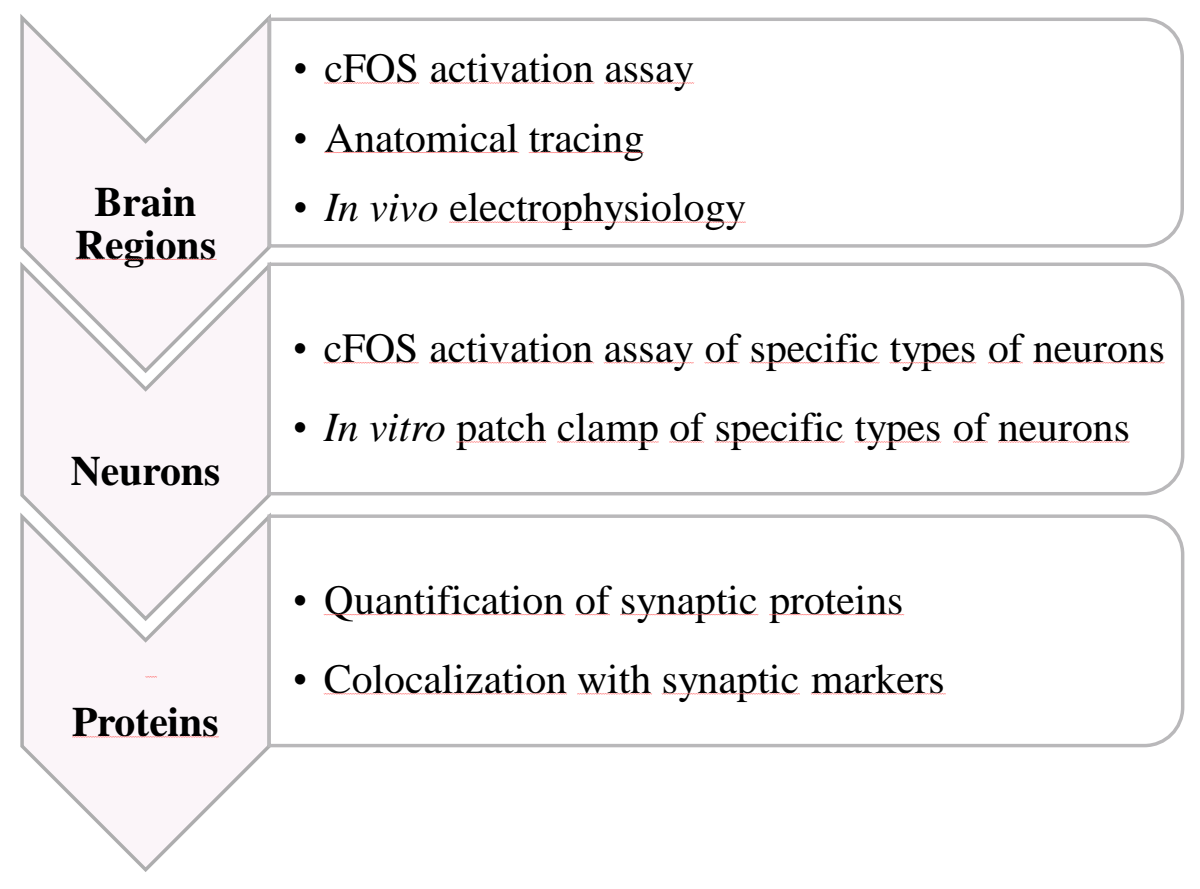

Figure 1.7| Framework for studying the role of Nlgn2 and IgSF9b in anxiety- related brain regions. 


\section{MATERIALS AND METHODS}

\subsection{Experimental Animals}

Nlgn2 KO mice (14) were generated in our laboratory on an $129 / \mathrm{Sv}$ background and were backcrossed onto a C57BL/6J background for at least six generations. Male wildtype (WT), heterozygous (Het) and homozygous (KO) littermates were obtained from Nlgn2 heterozygous breeding pairs. IgSF9b KO mice were obtained from Lexicon Pharmaceuticals (The Woodlands, TX, U.S.A.; Omnibank clone 281214, generated through insertion of the Omnibank gene trap vector 48 into the IgSF9b gene in Sv129 ES cells) and were backcrossed onto a C57BL/6J background for at least six generations. Nlgn2 KO mice and IgSF9b KO mice were crossed to generate Nlgn2 het x IgSF9b het breeding pairs, from which four experimental genotypes were obtained as littermates, i.e. WT, Nlgn2 KO, IgSF9b KO and double $\mathrm{KO}$ mice. For in vitro electrophysiology experiments, these mice were additionally crossed with mice expressing a Venus transgene under the control of the VGAT promoter to label inhibitory interneurons (Wang et al. 2009, Neuroscience 164:1031-1043). All mice were 2-3 months old at the beginning of the experiment. Animals were maintained on a 12 hour light/dark cycle, with food and water ad libitum. All experiments were performed during the light cycle (with the exception of home cage activity monitoring of Nlgn2 $\mathrm{KO}$ mice as described below). The experimenter was blind to genotype during all stages of data acquisition and analysis. All procedures were carried out in agreement with the guidelines for the welfare of experimental animals issued by the Federal Government of Germany and the Max Planck Society.

\subsection{Behavioral Characterization}

Assessment of anxiety. To assess anxiety levels, cohorts of Nlgn2 KO, Nlgn2 Het and WT mice were subjected to the following three anxiety tests on consecutive days: Elevated plus maze (EPM), open field test (OFT) and light/dark test (LDT). These tests are well- validated anxiety assays (Carola et al. 2002) and measure the extent of avoidance of naturally aversive environments: the open arms of elevated plus maze (EPM), the center of the open field chamber (OFT) and the brightly lit areas (LDT). The following parameters were assessed: EPM, total distance travelled in 
MATERIALS AND METHODS

the open and closed arms, distance travelled in the open arms, number of entries to the open arms, time spent in the open arms; OFT, total distance travelled in the OF chamber, distance travelled in the center zone, distance travelled in the periphery zone, number of entries to the center zone, time spent in the center zone; LD, distance travelled in the light zone, number of entries to the light zone, time spent in the light zone.

To assess anxiety levels in Nlgn2 KO, IgSF9b KO, double KO and WT mice, exclusively OFT was used. Each group of four male or female mice including one mouse of each genotype was tested on a separate day. The following parameters were assessed: total distance travelled in the OF chamber, distance travelled in the center zone, distance travelled in the periphery zone, number of entries to the center zone, time spent in the center zone, distance travelled in the intermediate zone, number of entries to the intermediate zone, time spent in the intermediate zone.

Detailed parameters for each test are listed in Table 2.1. Performance was recorded using an overhead camera system and scored automatically using the Viewer software (Biobserve, Germany).

Table 2.1| Parameters for anxiety measurements

\begin{tabular}{|c|c|c|c|c|}
\hline Test & Dimensions of chamber & $\begin{array}{l}\text { Light } \\
\text { intensity }\end{array}$ & $\begin{array}{l}\text { Placement of } \\
\text { mouse }\end{array}$ & $\begin{array}{l}\text { Duration of } \\
\text { assessment }\end{array}$ \\
\hline $\begin{array}{l}\text { Elevated } \\
\text { plus maze }\end{array}$ & $\begin{array}{l}\text { Two open arms } 28 \times 5 \mathrm{~cm} \\
\text { Two closed arms } 28 \times 5 \mathrm{~cm} \text { (walls } 15 \mathrm{~cm} \text { ) } \\
\text { Center zone } 5 \times 5 \mathrm{~cm}\end{array}$ & $90 \operatorname{lux}$ & Closed arm & $5 \mathrm{~min}$ \\
\hline $\begin{array}{l}\text { Open field } \\
\text { test }\end{array}$ & $\begin{array}{l}\text { Arena } 50 \times 50 \mathrm{~cm} \text { (walls } 50 \mathrm{~cm} \text { high) } \\
\text { Center zone } 25 \times 25 \mathrm{~cm} \\
\text { Intermediate zone surrounding the center } \\
\text { zone } 6.25 \mathrm{~cm} \text { at each direction }\end{array}$ & $20 \operatorname{lux}$ & $\begin{array}{l}\text { Corner of } \\
\text { periphery }\end{array}$ & $10 \mathrm{~min}$ \\
\hline $\begin{array}{l}\text { Light/dark } \\
\text { test }\end{array}$ & $\begin{array}{l}\text { Open (light) compartment } 25 \times 25 \mathrm{~cm} \\
\text { Closed (dark) compartment } 25 \times 25 \mathrm{~cm}\end{array}$ & $140 \operatorname{lux}$ & $\begin{array}{l}\text { Closed } \\
\text { compartment }\end{array}$ & $5 \min$ \\
\hline
\end{tabular}


MATERIALS AND METHODS

Assessment of home cage activity. Recording of home cage activity was performed using the LABORAS system and software (Metris, Netherlands). The following aspects of activity were recorded: total distance travelled and duration of locomotion, immobility, climbing, grooming and circling. A detailed description of the assessment of home cage activity can be found in table 2.2.

Table 2.2| Measurement of home cage activity

Day Description

1,2 Habituation to the room and to the cage, in individual cages $(20 \times 25 \mathrm{~cm})$
Duration

17:00 - 09:00, dark cycle: 19:00 - 07:00 h

3 Transfer of the cages onto vibration sensitive platforms and recording of activity with LABORAS (Metris, Hoofddorp, The Netherlands)

Assessment of freezing in a novel environment. Mice were placed inside a fear conditioning chamber (25 x $30 \mathrm{~cm}$, Med Associates) with a camera fixed on one of the internal walls. Freezing behavior was recorded for a period of 2 minutes and scored automatically using the Video Freeze software (Med Associates) with the motion detection threshold set to 60 .

\subsection{In vitro Electrophysiology}

Preparation of slices. Adult (8-12 weeks) mice were anesthetized with Avertin and perfused transcardially for 90 seconds with ice-cold sucrose solution, as described for the preparation of amygdala slices taken from adult animals (Adhikari et al. 2015). The brains were rapidly dissected and placed in the same sucrose solution, the brainstem dissected and the brains mounted on holder and transferred to vibrotome chamber filled with sucrose solution. After slicing, $300 \mu \mathrm{m}$ coronal sections containing basal and central amygdala were transferred to a holding chamber filled with artificial cerebrospinal fluid (aCSF) equilibrated with $95 \% \quad \mathrm{O}_{2} / 5 \% \mathrm{CO}_{2}$. Slices were allowed to recover for 20 minutes at $33 \mathrm{C}^{\circ}$ and then maintained at the room temperature. 
Patch clamp recording. Whole-cell patch clamp recordings were obtained at room temperature $\left(22 \mathrm{C}^{\circ}\right)$ with EPC10 amplifier. Slices were kept in a recording chamber and perfused with aCSF at the rate of 1-2 $\mathrm{ml} / \mathrm{min}$. Neurons were visually identified with infrared video microscopy using an upright microscope equipped with a $60 \mathrm{X}$ objective. Patch electrodes (resistance then filled with internal solution was 3-5 M $\Omega$ ) were pulled from borosilicate glass tubes. For voltage clamp experiments to record miniature inhibitory post-synaptic currents (mIPSCs), patch electrodes were filled with Cs- based internal solution. To exclude glutamatergic inputs, NBQX (6-cyano-7nitroquinoxaline-2,3-dione, $2 \mu \mathrm{M}$ : AMPA receptor antagonist) and A - CPP ((RS)-3-(2Carboxypiperazin-4-yl)-propyl-1-phosphonic acid, $2 \mu \mathrm{M}$ : NMDA receptor antagonist) were added to the aCSF. To exclude action potential dependent IPSCs, tetrodotoxin (TTX, $1 \mu \mathrm{M})$ was added to the ACSF. Whole cell patch-clamp recordings were included in analysis if initial access resistance was below $13 \mathrm{M} \Omega$ and did not change for more than 20 percent; and the leak current was below 200 pA during the recording. Data were acquired with Patchmaster software, filtered at $2 \mathrm{kHz}$ and digitised at $20 \mathrm{kHz}$. Data was analysed in Igor Pro, using costume made algorithms of template matching to detect mini currents (Taschenberger et al. 2005).

\subsection{In vivo Electrophysiology}

Surgery Male mice were single housed for 7 days before the recording, and provided with food and water ad libitum. Experiments were performed during the light cycle. Mice were anesthetized with avertin (loading dose $2 \mathrm{~mL} / 100 \mathrm{~g}$, maintenance doze $0.2 \mathrm{~mL} / 100 \mathrm{~g}$ intraperitoneal). Anesthetized mice were placed in a stereotaxic frame and their body temperature was monitored by a rectal probe and maintained at $36^{\circ} \mathrm{C}$. An incision in the midline of the scalp was made to expose the skull. Bregma and lambda were aligned to a plane level $\pm 50 \mu \mathrm{m}$. Multi-wire electrode array was unilaterally implanted targeting the left central medial nucleus $(0.9 \mathrm{~mm}$ posterior, 2.3 $\mathrm{mm}$ lateral and $5.04 \mathrm{~mm}$ ventral to the bregma).

The electrodes consisted of 2 bundles (spaced 750-950 $\mu \mathrm{m}$ ) of 8 individual insulated tungsten wires (13 $\mu \mathrm{m}$ inner diameter, impedance $60-100 \mathrm{k} \Omega)$ inserted into a polymide tube $(127 \mu \mathrm{m}$ inner diameter) and attached to an 18-pin connector. A reference screw was implanted above the cerebellum. The implant was secured with two screws implanted in the skull at $\sim 300 \mu \mathrm{m}$ lateral 
and anterior to the electrode and bonded with dental cement. Immediately after the surgery, mice received subcutaneously analgesic (Carprofen $5 \mathrm{mg} / \mathrm{kg}$ ) and antibiotic (Baytril $5 \mathrm{mg} / \mathrm{kg}$ ). 24 hours after the surgery, mice received Caprofen $(5 \mathrm{mg} / \mathrm{kg})$ subcutaneously and Baytril in the drinking water $(0.2 \mathrm{mg} / \mathrm{ml})$. Mice were sacrificed following the open field recording for histological verification of the recording site.

Data acquisition. The experimental design is summarized at figure 2.1. Electrophysiological signal was amplified and sent to acquisition board. The raw signal was acquired at $32 \mathrm{kHz}$ sampling rate, bandpass filtered $(0.1-9000 \mathrm{~Hz})$ and stored for offline analysis. During the experiment, simultaneous electrophysiological and video recordings were made by Cheetah Data Acquisition System (Figure 2.2).

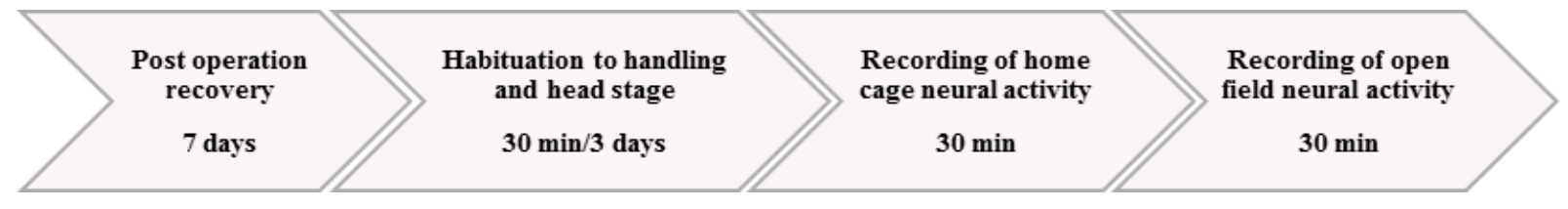

Figure 2.1| Stages of in vivo electrophysiology experiment.

Data analysis Local field potentials were analyzed in custom-written MATLAB scripts. The signal was filtered between 0.7 and $400 \mathrm{~Hz}$ using a zero-phase distortion FIR filter and down sampled to $1 \mathrm{kHz}$. For the power and coherence analysis we used the multitaper method (Chronux Package) (Mitra \& Bokil 2007), using 5 tapers (discrete prolate spheroidal sequences) with a time bandwidth product of 3 , and 0.1 seconds overlap.

For each mouse, we extracted the time periods and the trajectory of the movement in the periphery and center using a modified version of the autotyping toolbox (Patel et al. 2014). For the power correlations as a function of time spent in the center, first we summed the power 

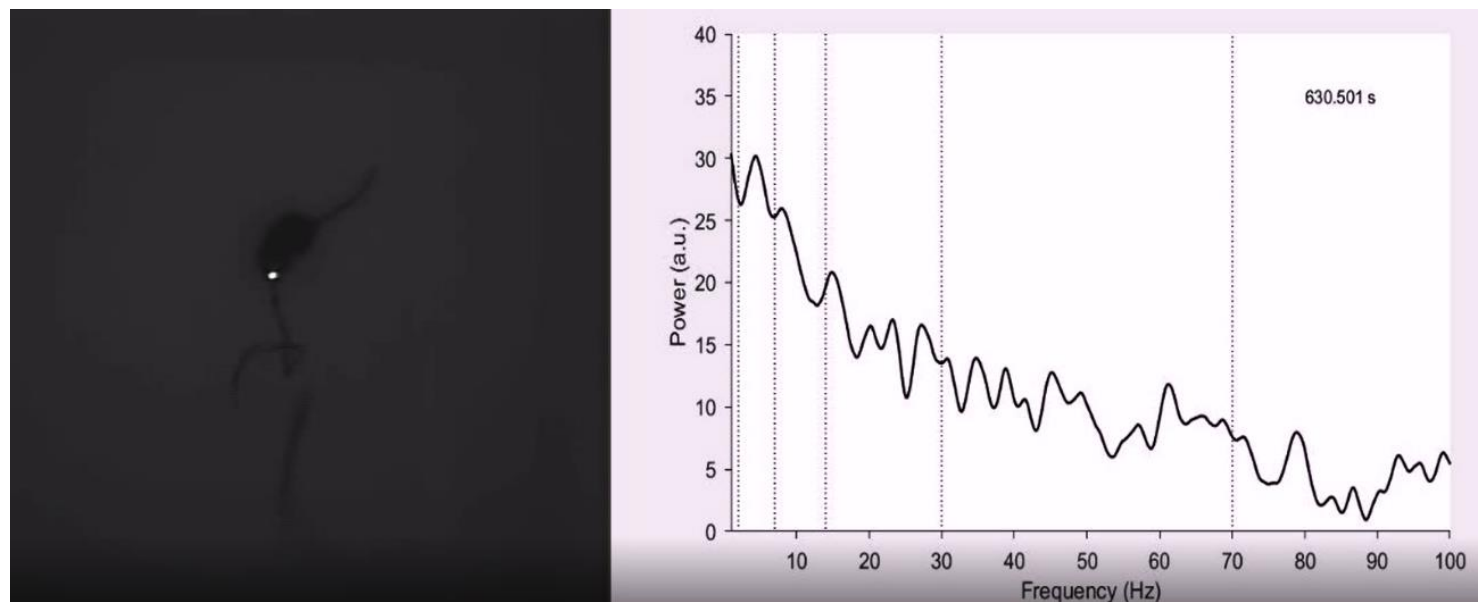

Figure 2.2| In vivo recording of CeM activity. Mouse in the open field during recording session (left) and an example of power spectrum of local field potential obtained during the exploration of the open field (right).

across the gamma frequency band $(70-120 \mathrm{~Hz})$, and then we averaged the summed values across all the time points spent either in the center or periphery. Using this average power values obtained from the periphery and the center we computed the ratio (center/periphery) for each mouse and calculated the linear correlation coefficient (fit function, MATLAB) including mice from all genotypes.

To evaluate power changes during transition periods from periphery to the center, we extracted all the trials at which the mouse shuttled from the periphery to the center and back to periphery during $\leq 10$ seconds. Extracted trials were aligned so the time points at which mice were at the minimum distance from the center at each trial overlapped. The power values at each time point were z-score transformed to a baseline defined as the initial 3 seconds of each trial as follows:

$$
\operatorname{zscore}(t)=\frac{P(t)-\overline{\text { baseline }}}{\text { std }(\text { baseline })}
$$

Where $z \operatorname{score}(t)$ is the z-score at each time point $t, P$ is the power value of a given frequency, $\overline{\text { baseline }}$ is the average power of the baseline and std(baseline) is the standard deviation of the baseline period. For the analysis of power change of the gamma band during transition periods, we average the z-scored values across the gamma range $(70-120 \mathrm{~Hz})$ at each time point. 


\subsection{Immunohistochemistry Procedures}

Paraformaldehyde perfusion fixation. Animals were anesthetized with Avertin (500 ul/25 gram) and perfused transcardially with $0.9 \%$ sodium chloride at $\mathrm{pH} 7.4$ for 1 minute, followed by perfusion with $4 \%$ paraformaldehyde diluted in $0.1 \mathrm{M}$ phosphate buffer (PB) for 8 minutes. The brains were dissected and post- fixed overnight at $4^{\circ} \mathrm{C}$ in $4 \%$ paraformaldehyde diluted in $0.1 \mathrm{M}$ PB. On the following day, the brains were transferred to $30 \%$ sucrose solution in $0.1 \mathrm{M}$ PB and kept for 1-2 days at $4{ }^{\circ} \mathrm{C}$ prior the slicing. The brains were sliced in cryostat at temperature of -18 $\mathrm{C}^{\circ}$. Free floating coronal slices were collected in PBS. The slices were washed in phosphate buffer saline (PBS), blocked for one hour, incubated with primary and secondary antibodies (Table 2.7), mounted on glass slides using Aqua-Poly/Mount, dried overnight at $4 \mathrm{C}^{\mathrm{o}}$ and covered with glass coverslips. Sections were washed with PBS after each incubation. Blocking and staining conditions are specified in the table 2.3 .

Methanol fixation of fresh frozen brains. Animals were decapitated and the brains were dissected and rapidly frozen in an isopentane bath at $-38^{\circ} \mathrm{C}$. The brains were sliced in cryostat at temperature of $-18 \mathrm{C}^{\mathrm{o}}$. Coronal sections of amygdala were mounted on glass slides inside the cryostat and dried for 40 minutes at room temperature. Sections were then fixed in methanol at $20^{\circ} \mathrm{C}$ for 5 minutes, blocked for 1 hour, washed with PBS and incubated with primary and secondary antibodies (Table 2.7), dried overnight at $4 \mathrm{C}^{\mathrm{o}}$ and covered with glass coverslips. Blocking and staining conditions are specified in the table 2.3.

\section{6 cFOS Assay}

Measuring the expression of neuronal activity marker cFOS (Sagar et al. 1988) was used to assess the neuronal activity of brain regions involved in anxiety- related processing, including amygdala nuclei and prefrontal cortex (summarized at table 2.4).

To induce cFOS activation across components of anxiety- processing circuitry, mice were taken from their home cages and exposed to novel environment (open field chamber) for 10 minutes. After the exposure to the open field, mice were returned to their home cages and kept there for 90 minutes to allow for expression of cFOS. After 90 minutes in the home cages, mice were sacrificed 


\section{MATERIALS AND METHODS}

and perfused with 4\% PFA as described above. Brains were dissected and processed as described above for paraformaldehyde perfusion- fixed slices. The stages of cFOS activation assay are summarized at figure 2.3. Blocking and staining conditions for cFOS labelling are specified in the table 2.3 .

$\left\langle\begin{array}{c}\text { Habituation to testing room } \\ 3-4 \mathrm{hr}\end{array}\right\rangle \begin{gathered}\text { Open field test } \\ 10 \mathrm{~min}\end{gathered}\left|\begin{array}{c}\text { Home cage } \\ 90 \mathrm{~min}\end{array}\right\rangle$ Perfusion

Figure 2.3| Stages of cFOS assay.

Table 2.3| Immunolabeling conditions.

\begin{tabular}{|c|c|c|c|}
\hline Experiment overview & $\begin{array}{l}\text { Fixation } \\
\text { Method }\end{array}$ & Slicing & Blocking solution \\
\hline $\begin{array}{l}\text { - Co-labeling cFos and } \\
\text { Somatostatin } \\
\text {-Co-labeling cFos and } \\
\text { Parvalbumin/CAMKII } \\
\text {-Co-labeling VIAAT and Vglut2 }\end{array}$ & \multirow[t]{2}{*}{$\begin{array}{l}\text { Perfusion with } \\
4 \% \text { paraform- } \\
\text { aldehyde }\end{array}$} & \multirow[t]{2}{*}{$\begin{array}{l}\text { Free floating } \\
\text { coronal } \\
\text { sections, } \\
40 \mu \mathrm{m}\end{array}$} & $\begin{array}{l}10 \% \text { donkey serum and } \\
0.3 \% \text { Triton in PBS } \\
3 \% \text { BSA, } 10 \% \text { goat } \\
\text { serum and } 0.3 \% \text { Triton } \\
\text { in PBS }\end{array}$ \\
\hline -Labeling VGAT, cFos or GFP & & & \\
\hline $\begin{array}{l}\text {-Labeling Gephyrin, PSD-95, S- } \\
\text { SCAM, GABA }_{A} \alpha 1, \text { GABA } \\
\text { GABA }_{A} \alpha 2, \text { Nlgn2 or IgSF9b } \\
\text {-Co-labeling Nlgn2 with } \\
\text { Gephyrin/PSD-95 } \\
\text {-Co-labeling IgSF9b with Nlgn2/ } \\
\text { Gephyrin/ VGAT/ Vglut 2/ } \\
\text { Ankyrin G/PSD-95 }\end{array}$ & $\begin{array}{l}\text { Methanol } \\
\text { fixation of fresh- } \\
\text { frozen brains }\end{array}$ & $\begin{array}{l}\text { Coronal } \\
\text { sections, } \\
20 \mu \mathrm{m}, \\
\text { mounted on } \\
\text { slides before } \\
\text { blocking }\end{array}$ & $\begin{array}{l}3 \% \text { BSA, } 10 \% \text { goat } \\
\text { serum and } 0.3 \% \text { Triton } \\
\text { in PBS }\end{array}$ \\
\hline
\end{tabular}


MATERIALS AND METHODS

Table 2.4| Brain regions assesed in cFOS activation assay

\begin{tabular}{lll}
\hline Brain region & Subregions & Coordinates from bregma \\
\hline \hline Medial prefrontal cortex & Infralimbic cortex & $2.8 \mathrm{~mm}-1.94 \mathrm{~mm}$ \\
& Prelimbic cortex & \\
& Basal amygdala, anterior & $-0.94 \mathrm{~mm}--1.28 \mathrm{~mm}$ \\
Amygdala & Basal amygdala, posterior & $-1.28 \mathrm{~mm}--1.77 \mathrm{~mm}$ \\
& Central lateral amygdala & $-0.94 \mathrm{~mm}--1.64 \mathrm{~mm}$ \\
& & $-0.94 \mathrm{~mm}--1.64 \mathrm{~mm}$ \\
& Central medial amygdala & $-1.28 \mathrm{~mm}--1.77 \mathrm{~mm}$ \\
& & \\
& Lateral amygdala &
\end{tabular}

\subsection{Image Acquisition and Data Analysis}

General comments. Images were acquired using a confocal laser scanning microscope (Leica SP2). For each WT-KO pair, sections were anatomically matched and the settings for laser power, gain and offset were kept constant during imaging. Detailed description of acquisition parameters and analysis methods appears in table 2.5.

Imaging of synaptic markers. Images of synaptic markers (gephyrin, PSD- 95, Nlgn2 and VIAAT) were obtained using a 63x oil immersion objective. The number and size of particles, as well as mean fluorescence intensity, were quantified in the area surrounding the cell body, and the number of particles per area was divided by the length of the perimeter to obtain the final result, as demonstrated in figure 2.4. Colocalization of synaptic markers was analyzed using Imaris software using coloc function. 
A
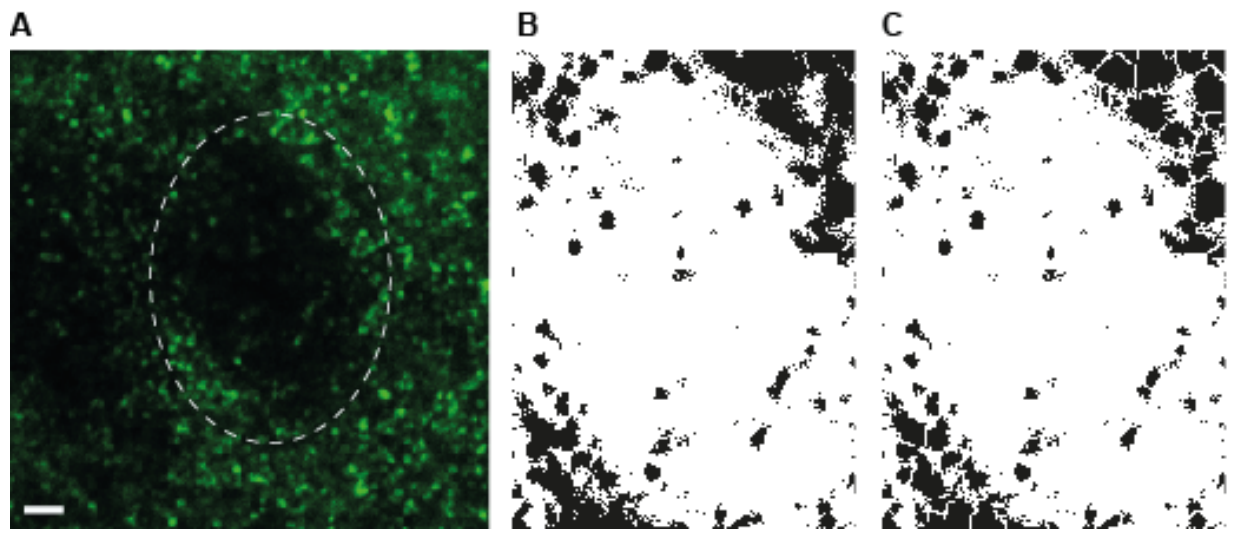

D
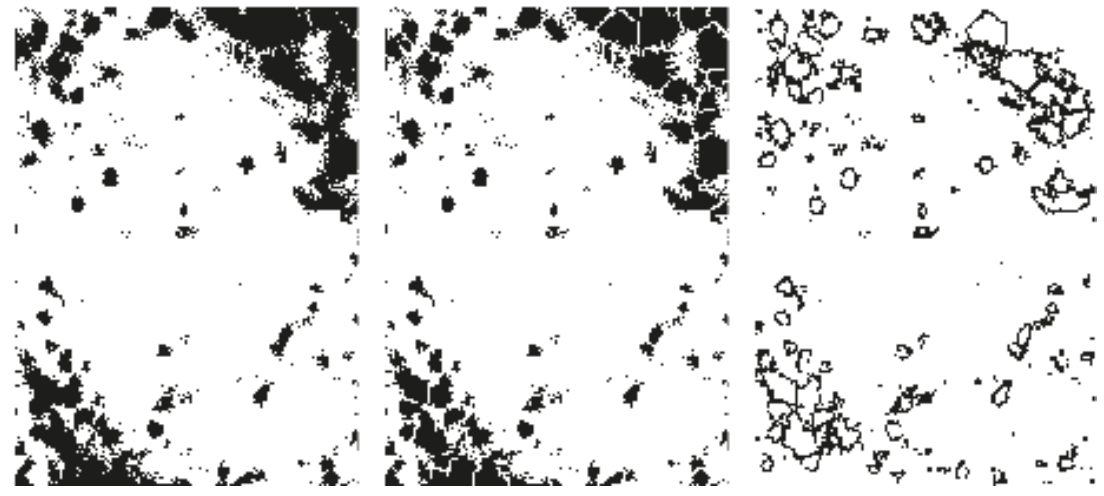

Figure 2.4| Stages in quantification of synaptic markers.

A. Photomicrograph of an optical section with delineated perimeter of the cell body.

B-C. Quantification of synaptic puncta in ImageJ- thresholding (B), applying segmentation algorithm to separate puncta (C) and applying "analyze particles" to outline and count the puncta (D).

Imaging of cFos, cellular markers and retrobeads. Images of cFos, cellular markers and retrobeads (see next section) were obtained using a 40x oil immersion objective as described in table 2.6. cFos images were thresholded manually in ImageJ with same threshold value for WT and Nlgn2 $\mathrm{KO}$ in each pair. cFos images were tresholded in ImageJ (Figure 2.5), and single- and double-labeled cells were then quantified using Imaris (Bitplane, Zurich, Switzerland) as demonstrated at figure 2.6.
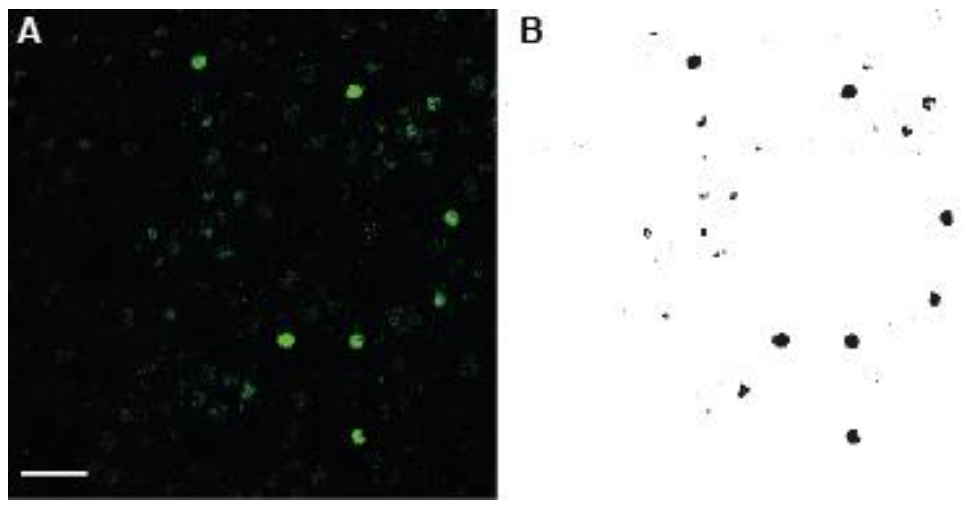

Figure 2.5| Thresholding in ImageJ. Photomicrograph of cFOS expression before (A) and after (B) thresholding in ImageJ. 

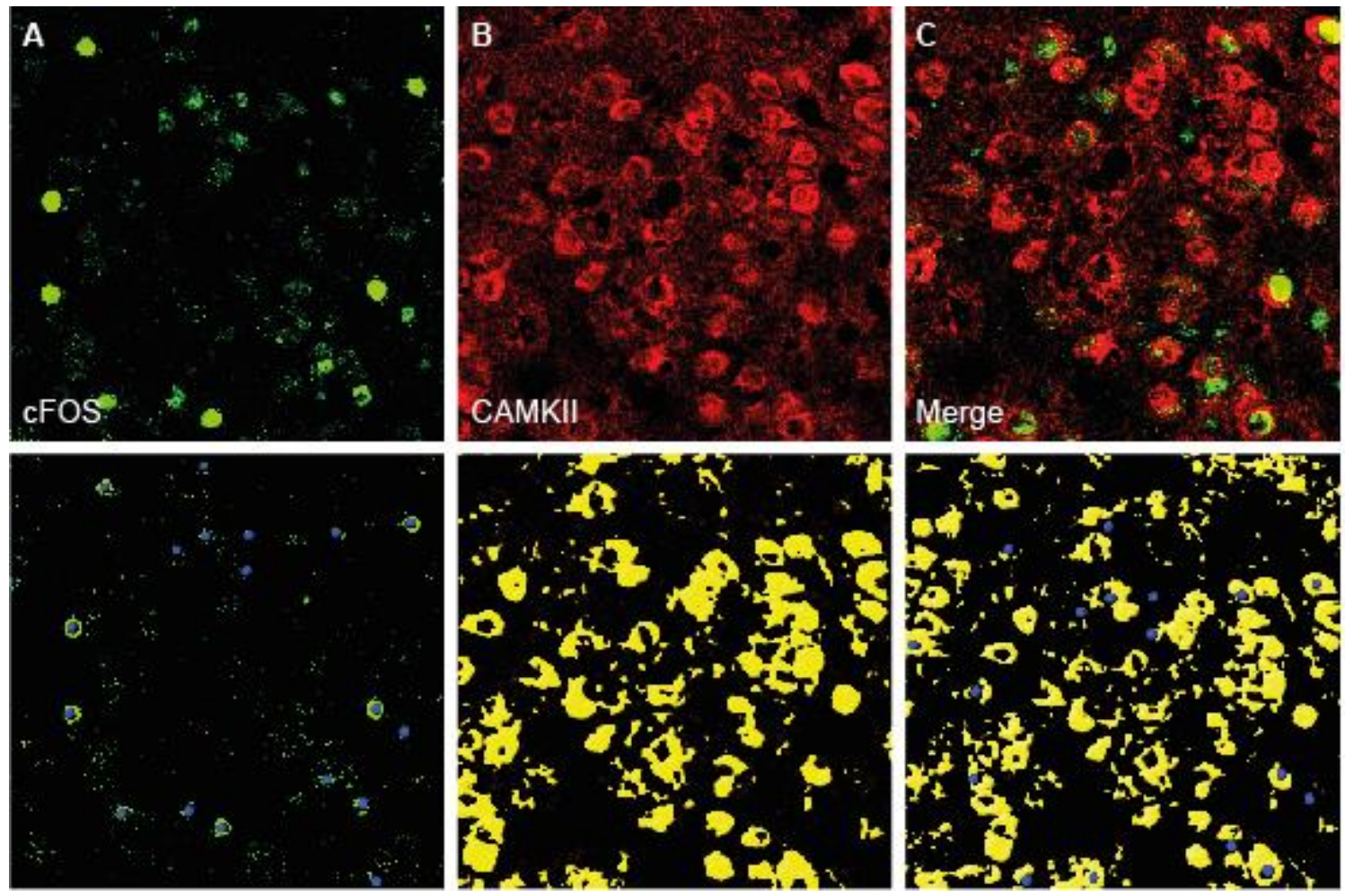

Figure 2.6| Colocalization of cFOS positive neurons with cellular markers in IMARIS.

A. Thersholded cFOS photomicrograph (upper image) and cFOS positive cells detected in IMARIS (lower image, blue spots)

B. CAMKII photomicrograph before (upper image) and after detection of CAMKII positive cells in IMARIS. "create surface" algorithm was used to mark the CAMKII positive cells (lower image, yellow spots).

C. cFOS spots with CAMKII surfaces before application of "find spots close to surfaces" algorithm in IMARIS. 
Table 2.5| Image acquisition and image analysis parameters

\begin{tabular}{|c|c|c|c|c|}
\hline Experiment & $\begin{array}{l}\text { Magnifi- } \\
\text { cation }\end{array}$ & $\begin{array}{l}\text { Stack thickness, } \\
\text { number of } \\
\text { sections }\end{array}$ & $\begin{array}{l}\text { Total number } \\
\text { of images/ } \\
\text { stacks/animal }\end{array}$ & $\begin{array}{l}\text { Thresholding } \\
\text { and analysis }\end{array}$ \\
\hline $\begin{array}{l}\text { Nlgn2/gephyrin } \\
\text { colocalization; } \\
\text { Nlgn2/PSD-95 } \\
\text { colocalization }\end{array}$ & $\begin{array}{l}63 \mathrm{x} \\
\text { Digital } \\
\text { zoom: } \\
8 \mathrm{x}\end{array}$ & N/A & $\begin{array}{l}3 \text { images (1 } \\
\text { image /slice) }\end{array}$ & $\begin{array}{l}\text { Automatic threshold and degree of } \\
\text { colocalization both assed with "coloc" } \\
\text { tool in IMARIS (Bitplane, Zurich, } \\
\text { Switzerland) }\end{array}$ \\
\hline $\begin{array}{l}\text { Quantification } \\
\text { of VIAAT and } \\
\text { gephyrin- } \\
\text { positive puncta }\end{array}$ & $\begin{array}{l}63 \mathrm{x} \\
\text { Digital } \\
\text { zoom: } \\
8 \mathrm{x}\end{array}$ & $\begin{array}{l}2 \mu \mathrm{m}, \\
2 \text { sections }\end{array}$ & $\begin{array}{l}18 \text { stacks } \\
\text { imaged from } \\
6 \text { slices }(3 \\
\text { stacks } / \text { slice) }\end{array}$ & $\begin{array}{l}\text { Thresholded manually in ImageJ, } \\
\text { quantification performed with "count } \\
\text { particles" module in ImageJ }\end{array}$ \\
\hline $\begin{array}{l}\text { Quantification } \\
\text { of PV-positive } \\
\text { puncta }\end{array}$ & $40 x$ & $\begin{array}{l}5 \mu \mathrm{m}, \\
2 \text { sections }\end{array}$ & $\begin{array}{l}10 \text { stacks } \\
\text { imaged from } \\
5 \text { slices }(2 \\
\text { stacks } / \text { slice })\end{array}$ & $\begin{array}{l}\text { Quantification with "create spots" tool } \\
\text { in IMARIS (Bitplane, Zurich, } \\
\text { Switzerland) }\end{array}$ \\
\hline $\begin{array}{l}\text { Quantification } \\
\text { of cFOS } \\
\text { positive cells, } \\
\text { baseline cFOS } \\
\text { expression }\end{array}$ & $40 x$ & $\begin{array}{l}10 \mu \mathrm{m}, \\
4 \text { sections }\end{array}$ & $\begin{array}{l}5 \text { stacks } \\
\text { imaged from } \\
5 \text { slices }(1 \\
\text { stack/slice })\end{array}$ & $\begin{array}{l}\text { Manual threshold set as } 3 * \text { background } \\
\text { intensity and thresholded in ImageJ, } \\
\text { quantification with "create spots" tool } \\
\text { in IMARIS (Bitplane, Zurich, } \\
\text { Switzerland) }\end{array}$ \\
\hline $\begin{array}{l}\text { Quantification } \\
\text { of cFOS } \\
\text { positive cells in } \\
\text { cortical regions }\end{array}$ & $40 x$ & $\begin{array}{l}10 \mu \mathrm{m}, \\
4 \text { sections }\end{array}$ & $\begin{array}{l}5 \text { stacks } \\
\text { imaged from } \\
5 \text { slices }(1 \\
\text { stack/slice })\end{array}$ & $\begin{array}{l}\text { Manual threshold set as } 3 * \text { background } \\
\text { intensity and thresholded in ImageJ, } \\
\text { quantification with "create spots" tool } \\
\text { in IMARIS (Bitplane, Zurich, } \\
\text { Switzerland) }\end{array}$ \\
\hline $\begin{array}{l}\text { Quantification } \\
\text { of cFOS in } \\
\text { basal amygdala }\end{array}$ & $40 x$ & $\begin{array}{l}5 \mu \mathrm{m}, \\
2 \text { sections }\end{array}$ & $\begin{array}{l}10 \text { stacks } \\
\text { imaged from } \\
5 \text { slices }(2 \\
\text { stacks } / \text { slice })\end{array}$ & $\begin{array}{l}\text { Manual threshold set as } 3 * \text { background } \\
\text { intensity and thresholded in ImageJ, } \\
\text { quantification with "create spots" tool } \\
\text { in IMARIS (Bitplane, Zurich, } \\
\text { Switzerland) }\end{array}$ \\
\hline $\begin{array}{l}\text { Quantification } \\
\text { of PV and SOM } \\
\text { positive cells }\end{array}$ & $40 x$ & $\begin{array}{l}5 \mu \mathrm{m}, \\
2 \text { sections }\end{array}$ & $\begin{array}{l}10 \text { stacks } \\
\text { imaged from } \\
5 \text { slices }(2 \\
\text { stacks } / \text { slice })\end{array}$ & $\begin{array}{l}\text { Application of "median filter" tool to } \\
\text { the images, and quantification using } \\
\text { "create spots" tool, both with IMARIS } \\
\text { (Bitplane, Zurich, Switzerland) }\end{array}$ \\
\hline $\begin{array}{l}\text { Quantification } \\
\text { of CAMKII } \\
\text { positive cells }\end{array}$ & $40 x$ & $\begin{array}{l}2 \mu \mathrm{m}, \\
2 \text { sections }\end{array}$ & $\begin{array}{l}16 \text { stacks } \\
\text { imaged from } \\
8 \text { slices }(2 \\
\text { stacks } / \text { slice })\end{array}$ & $\begin{array}{l}\text { Application of "median filter" tool to } \\
\text { the images, and quantification using } \\
\text { "create spots" tool, both with IMARIS } \\
\text { (Bitplane, Zurich, Switzerland) }\end{array}$ \\
\hline
\end{tabular}

Colocalization of cFOS and PV and SOM
Imaged and thresholded as described above for cFOS and each marker, colocalized using "colocalize spots" tool in IMARIS (Bitplane, Zurich, Switzerland) for PV and SOM colocalization and "find spots close to surface" for CAMKII and retro beads colocalization 


\subsection{Retrograde Labeling of BA Neurons That Project to CeM}

Surgical procedure. Mice received intraperitoneal injection of Caprofen $(5 \mathrm{mg} / \mathrm{kg})$ to reduce postsurgery pain 30 minutes prior to surgery. Mice were anaesthetized with Avertin by intraperitoneal injection (500 ul Avertin/25g body weight) and placed in digital stereotaxic frame. To bilaterally label BA neurons projecting to the CeM, $50 \mathrm{nl}$ of red retrobeads (excitation maximum at $530 \mathrm{~nm}$ and emission maximum at $590 \mathrm{~nm})$ were injected into the CeM $(0.70 \mathrm{~mm}$ posterior, $\pm 2.35 \mathrm{~mm}$ medial and $5.08 \mathrm{~mm}$ ventral from bregma). A Hamilton syringe (1 ul) was used to manually deliver the retrobeads at the rate of $0.5 \mathrm{~nL} / \mathrm{s}$. After the injection was completed, the tip of the syringe was raised for $100 \mu \mathrm{m}$ and left for 3 minutes to allow diffusion of the retrobeads at the injection site; and then slowly withdrawn at the rate of $1 \mathrm{~mm} /$ minute. Following surgery, mice received Metamizol with drinking water (200 mg/kg/day, drinking rate estimated at $3 \mathrm{ml} /$ day) for 3 days to reduce pain and risk of inflammation.

Data acquisition and analysis. Mice were single housed for 7 days post-surgery to allow their full recovery and travelling of beads from the injection site up the axons of BA neurons to their somata. To induce anxiety associated activation of basal amygdala, mice were subjected to open field test with consecutive cFOS activation assay as described above. Only slices from brains in which injection sites did not exceed the borders of CeM (confirmed by visualizing the sites of injection on 5 subsequent coronal sections of amygdala spanning $400 \mu \mathrm{m}$ of tissue, FigureX) were included in subsequent imaging and analysis. Data acquisition and quantification of cFOS positive cell bodies containing retrobeads was performed similarly to quantification of CAMKII - cFOS double labeled neurons as described above.
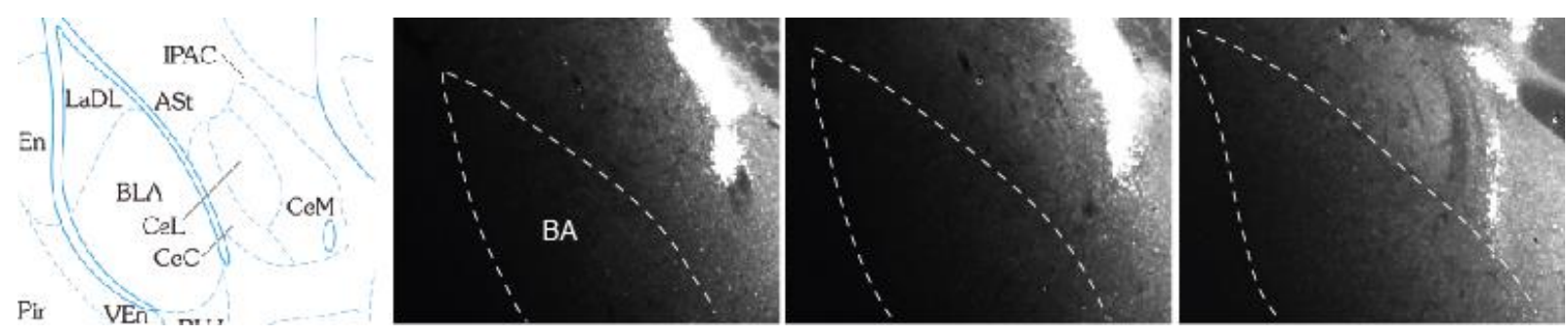

Figure 2.7| Verification of retro beads injection site. Serial photomicrographs of amygdala slices with injection site in CeM (bright mark, right images) as compared to anatomical atlas (left image, adapted from Franklin and Paxinos, 2007). 
MATERIALS AND METHODS

\subsection{Statistical Analysis}

All data were analyzed statistically using Prism (GraphPad Software, USA). Outliers, defined as two standard deviations from the mean, were removed prior to statistical analysis (maximum one outlier per group per experiment was detected). All the statistical tests conducted are summarized in table 2.6.

Table 2.6| Statistical analysis

\section{$\begin{array}{lll}\text { Experiment } & \text { Statistical test } & \text { Post-hoc test }\end{array}$}

\section{Results- Part 1}

Behavioral characterization

Quantification of cFOS and cellular markers

Quantification of synaptic markers

Patch clamp recording

\section{Results- Part 2}

Behavioral characterization Two- way ANOVA

Quantification of cFOS and cellular markers

Quantification of synaptic Two-way ANOVA markers

Patch clamp recording

Local field potentials recording in vivo
One-way ANOVA

Two-way ANOVA

Two-way ANOVA

Two-way repeated measures ANOVA
Tukey's test

Two- tailed paired Student's t-test

Two- tailed paired Student's t-test

Two- tailed paired Student's t-test

One- way ANOVA with Bonferroni's correction

Two- tailed paired Student's t-test

Two- tailed paired Student's t-test

Two- tailed paired Student's t-test

Kolmogorov-Smirnov test

Two- tailed paired Student's t-test 
MATERIALS AND METHODS

2.10 List of Antibodies

Table 2.7| Primary and secondary antibodies.

\begin{tabular}{|c|c|c|c|c|c|c|}
\hline \multirow[t]{2}{*}{ Antigen } & \multicolumn{3}{|c|}{$\underline{\text { Primary antibody }}$} & \multicolumn{3}{|c|}{$\underline{\text { Secondary antibody }}$} \\
\hline & Species & $\begin{array}{l}\text { Dilution, } \\
\text { duration of } \\
\text { incubation }\end{array}$ & Source & Species & $\begin{array}{l}\text { Dilution, } \\
\text { duration of } \\
\text { incubation }\end{array}$ & Source \\
\hline $\begin{array}{l}\text { Ankyrin } \\
\mathrm{G}\end{array}$ & $\begin{array}{l}\text { Rabbit } \\
\text { polyclonal }\end{array}$ & $\begin{array}{l}1: 1000 \\
12 \mathrm{~h}\end{array}$ & $\begin{array}{l}\text { Santa } \\
\text { Cruz } \\
\text { Biotech., } \\
\text { Dallas, } \\
\text { TX, USA }\end{array}$ & $\begin{array}{l}\text { Goat anti- } \\
\text { rabbit } \\
\text { A488 }\end{array}$ & $\begin{array}{l}1: 600 \\
2-\mathrm{h} 3 \mathrm{~h}\end{array}$ & $\begin{array}{l}\text { Invitrogen } \\
\text { Eugene, } \\
\text { OR, USA }\end{array}$ \\
\hline CAMKII & $\begin{array}{l}\text { Mouse } \\
\text { monoclonal } \\
\text {, clone 6G9 }\end{array}$ & $\begin{array}{l}1: 2000 \\
60 \mathrm{~h}\end{array}$ & $\begin{array}{l}\text { Abcam } \\
\text { Cambridg } \\
\text { e, MA, } \\
\text { USA }\end{array}$ & $\begin{array}{l}\text { Goat anti- } \\
\text { mouse } \\
\text { A555 }\end{array}$ & $\begin{array}{l}1: 600 \\
2-3 \mathrm{~h}\end{array}$ & $\begin{array}{l}\text { Invitrogen } \\
\text { Eugene, } \\
\text { OR, USA }\end{array}$ \\
\hline \multirow[t]{2}{*}{ cFOS } & $\begin{array}{l}\text { Rabbit } \\
\text { polyclonal, } \\
\text { clone SC52 }\end{array}$ & $\begin{array}{l}1: 2000 \\
12 \mathrm{~h}\end{array}$ & $\begin{array}{l}\text { Santa } \\
\text { Cruz } \\
\text { Biotech., } \\
\text { Dallas, } \\
\text { TX, USA }\end{array}$ & $\begin{array}{l}\text { Goat anti- } \\
\text { rabbit } \\
\text { A488 }\end{array}$ & $\begin{array}{l}1: 600 \\
2-3 \mathrm{~h}\end{array}$ & $\begin{array}{l}\text { Invitrogen } \\
\text { Eugene, } \\
\text { OR, USA }\end{array}$ \\
\hline & & & & $\begin{array}{l}\text { *Donkey } \\
\text { anti-rabbit } \\
\text { A555 }\end{array}$ & $\begin{array}{l}1: 600 \\
2-3 \mathrm{~h}\end{array}$ & $\begin{array}{l}\text { Mobitec, } \\
\text { Goettingen } \\
\text { Germany }\end{array}$ \\
\hline $\begin{array}{l}\mathrm{GABA}_{\mathrm{A}} \mathrm{R} \\
\alpha 1\end{array}$ & $\begin{array}{l}\text { Rabbit, } \\
\text { polyclonal }\end{array}$ & $\begin{array}{l}1: 1000 \\
12 \mathrm{~h}\end{array}$ & $\begin{array}{l}\text { Synaptic } \\
\text { Systems, } \\
\text { Göttingen, } \\
\text { Germany }\end{array}$ & $\begin{array}{l}\text { Goat anti- } \\
\text { rabbit } \\
\text { A488 }\end{array}$ & $\begin{array}{l}1: 600 \\
2-3 \mathrm{~h}\end{array}$ & $\begin{array}{l}\text { Invitrogen, } \\
\text { Eugene, } \\
\text { OR, USA }\end{array}$ \\
\hline $\begin{array}{l}\mathrm{GABA}_{\mathrm{A}} \mathrm{R} \\
\alpha 2\end{array}$ & $\begin{array}{l}\text { Rabbit, } \\
\text { polyclonal }\end{array}$ & $\begin{array}{l}1: 1000, \\
12 \mathrm{~h}\end{array}$ & $\begin{array}{l}\text { Synaptic } \\
\text { Systems, } \\
\text { Göttingen, } \\
\text { Germany }\end{array}$ & $\begin{array}{l}\text { Goat anti- } \\
\text { rabbit } \\
\text { A488 }\end{array}$ & $\begin{array}{l}1: 600 \\
2-3 \mathrm{~h}\end{array}$ & $\begin{array}{l}\text { Invitrogen, } \\
\text { Eugene, } \\
\text { OR, USA }\end{array}$ \\
\hline $\begin{array}{l}\mathrm{GABA}_{\mathrm{A}} \mathrm{R} \\
\gamma 2\end{array}$ & $\begin{array}{l}\text { Guinea pig, } \\
\text { polyclonal }\end{array}$ & $\begin{array}{l}1: 1000 \\
12 \mathrm{~h}\end{array}$ & $\begin{array}{l}\text { Fritschi } \\
\text { lab }\end{array}$ & $\begin{array}{l}\text { Goat anti- } \\
\text { guinea pig } \\
\text { A555 }\end{array}$ & $\begin{array}{l}1: 600 \\
2-3 \mathrm{~h}\end{array}$ & $\begin{array}{l}\text { Invitrogen, } \\
\text { Eugene, } \\
\text { OR, USA }\end{array}$ \\
\hline
\end{tabular}


MATERIALS AND METHODS

\begin{tabular}{|c|c|c|c|c|c|c|}
\hline Gephyrin & $\begin{array}{l}\text { Mouse } \\
\text { monoclonal } \\
\text { clone 3B11 }\end{array}$ & $\begin{array}{l}1: 1000, \\
12 \mathrm{~h}\end{array}$ & $\begin{array}{l}\text { Synaptic } \\
\text { Systems, } \\
\text { Göttingen, } \\
\text { Germany }\end{array}$ & $\begin{array}{l}\text { Goat anti- } \\
\text { mouse } \\
\text { A555 }\end{array}$ & $\begin{array}{l}1: 600 \\
2-3 \mathrm{~h}\end{array}$ & $\begin{array}{l}\text { Invitrogen, } \\
\text { Eugene, } \\
\text { OR } \\
\text { USA }\end{array}$ \\
\hline GFP & $\begin{array}{l}\text { Rabbit } \\
\text { polyclonal }\end{array}$ & $\begin{array}{l}1: 500 \\
12 \mathrm{~h}\end{array}$ & $\begin{array}{l}\text { MBL, } \\
\text { Nagoya, } \\
\text { Japan }\end{array}$ & $\begin{array}{l}\text { Goat anti- } \\
\text { mouse } \\
\text { A555 }\end{array}$ & $\begin{array}{l}1: 600 \\
2-3 \mathrm{~h}\end{array}$ & $\begin{array}{l}\text { Invitrogen, } \\
\text { Eugene, } \\
\text { OR, USA }\end{array}$ \\
\hline IgSF9b & $\begin{array}{l}\text { Rabbit } \\
\text { polyclonal, } \\
\text { clone }\end{array}$ & $\begin{array}{l}1: 1000, \\
12 \mathrm{~h}\end{array}$ & $\begin{array}{l}\text { Sigma- } \\
\text { Aldrich, } \\
\text { Munich, } \\
\text { Germany }\end{array}$ & $\begin{array}{l}\text { Goat anti- } \\
\text { rabbit } \\
\text { A488 }\end{array}$ & $\begin{array}{l}1: 600 \\
2-3 \mathrm{~h}\end{array}$ & $\begin{array}{l}\text { Invitrogen, } \\
\text { Eugene, } \\
\text { OR, USA }\end{array}$ \\
\hline Nlgn2 & $\begin{array}{l}\text { Rabbit } \\
\text { polyclonal }\end{array}$ & $\begin{array}{l}1: 500 \\
12 \mathrm{~h}\end{array}$ & Brose lab & $\begin{array}{l}\text { Goat anti- } \\
\text { mouse } \\
\text { A555 }\end{array}$ & $\begin{array}{l}1: 600 \\
2-3 \mathrm{~h}\end{array}$ & $\begin{array}{l}\text { Invitrogen, } \\
\text { Eugene, } \\
\text { OR, USA }\end{array}$ \\
\hline $\begin{array}{l}\text { Parvalbu- } \\
\text { min }\end{array}$ & $\begin{array}{l}\text { Mouse } \\
\text { monoclonal } \\
\text { clone } \\
\text { PV235 }\end{array}$ & $\begin{array}{l}1: 4000 \\
12 \mathrm{~h}\end{array}$ & $\begin{array}{l}\text { SWANT, } \\
\text { Bellinzon } \\
\text { a, } \\
\text { Switzerlan } \\
\text { d }\end{array}$ & $\begin{array}{l}\text { Goat anti- } \\
\text { mouse } \\
\text { A555 }\end{array}$ & $\begin{array}{l}1: 600 \\
2-3 \mathrm{~h}\end{array}$ & $\begin{array}{l}\text { Invitrogen, } \\
\text { Eugene, } \\
\text { OR, USA }\end{array}$ \\
\hline PSD-95 & $\begin{array}{l}\text { Mouse } \\
\text { monoclonal } \\
\text { clone } \\
\text { K28/43 }\end{array}$ & $\begin{array}{l}1: 2000 \\
12 \mathrm{~h}\end{array}$ & $\begin{array}{l}\text { Neuromab } \\
\text { UC Davis, } \\
\text { CA, USA }\end{array}$ & $\begin{array}{l}\text { Goat anti- } \\
\text { mouse } \\
\text { A555 }\end{array}$ & $\begin{array}{l}1: 600 \\
2-3 \mathrm{~h}\end{array}$ & $\begin{array}{l}\text { Invitrogen, } \\
\text { Eugene, } \\
\text { OR, USA }\end{array}$ \\
\hline SOM & $\begin{array}{l}\text { Goat } \\
\text { polyclonal, } \\
\text { clone } \\
\text { SC7819 }\end{array}$ & $\begin{array}{l}1: 500 \\
12 \mathrm{~h}\end{array}$ & $\begin{array}{l}\text { Santa } \\
\text { Cruz } \\
\text { Biotech- } \\
\text { nologies, } \\
\text { Dallas, } \\
\text { TX, USA }\end{array}$ & $\begin{array}{l}\text { Donkey } \\
\text { anti-goat } \\
\text { A488 }\end{array}$ & $\begin{array}{l}1: 600 \\
2-3 \mathrm{~h}\end{array}$ & $\begin{array}{l}\text { Mobitec, } \\
\text { Göttingen } \\
\text { Germany }\end{array}$ \\
\hline S-SCAM & $\begin{array}{l}\text { Rabbit } \\
\text { polyclonal }\end{array}$ & $\begin{array}{l}1: 1000, \\
12 \mathrm{~h}\end{array}$ & $\begin{array}{l}\text { Sigma- } \\
\text { Aldrich, } \\
\text { Munich, } \\
\text { Germany }\end{array}$ & $\begin{array}{l}\text { Goat anti- } \\
\text { rabbit } \\
\text { A555 }\end{array}$ & $\begin{array}{l}1: 600 \\
2-3 \mathrm{~h}\end{array}$ & $\begin{array}{l}\text { Invitrogen, } \\
\text { Eugene, } \\
\text { OR, USA }\end{array}$ \\
\hline Vglut 2 & $\begin{array}{l}\text { Guinea pig, } \\
\text { polyclonal }\end{array}$ & $\begin{array}{l}1: 1000 \\
36 \mathrm{~h}\end{array}$ & $\begin{array}{l}\text { Synaptic } \\
\text { Systems, } \\
\text { Göttingen, } \\
\text { Germany }\end{array}$ & $\begin{array}{l}\text { Goat anti- } \\
\text { guinea pig } \\
\text { A555 }\end{array}$ & $\begin{array}{l}1: 600 \\
2-3 \mathrm{~h}\end{array}$ & $\begin{array}{l}\text { Invitrogen, } \\
\text { Eugene, } \\
\text { OR, USA }\end{array}$ \\
\hline
\end{tabular}

Table 2.7 continued 
MATERIALS AND METHODS

$\begin{array}{lllllll}\text { VIAAT } & \text { Rabbit } & 1: 1000, & \text { Synaptic } & \text { Goat anti- } & 1: 600, & \text { Invitrogen, } \\ & \begin{array}{l}\text { Polyclonal, } \\ \text { clone }\end{array} & 36 \mathrm{~h} & \text { Systems, } & \text { rabbit } & 2-3 \mathrm{~h} & \text { Eugene, } \\ & & & \text { Göttingen, } & \text { A488 } & & \text { OR, USA } \\ & 131003 & & \text { Germany } & & \end{array}$

2.11 Solutions

Immunohistochemistry

Phosphate buffer saline (PBS)

$160 \mathrm{~g}$ of $\mathrm{NaCl}, 4.0 \mathrm{~g} \mathrm{KCl}, 36.1 \mathrm{~g}$ of $\mathrm{Na}_{2} \mathrm{HPO}_{4} * 2 \mathrm{H}_{2} \mathrm{O}, 4.8 \mathrm{KH}_{2} \mathrm{PO}_{4}$ in DDW; total volume 2L;

$\mathrm{pH}=7.4$.

Phosphate buffer $0.2 \mathrm{M}$ (PB)

27.3g of $\mathrm{Na}_{2} \mathrm{HPO}_{4}, 7.36 \mathrm{~g}$ of $\mathrm{NaH}_{2} \mathrm{PO}_{4}$; total volume $1000 \mathrm{~mL} ; \mathrm{pH}=7.4$.

Sucrose solution $30 \%$

$30 \mathrm{~g}$ of sucrose in $0.1 \mathrm{M} \mathrm{PB}$; total volume $100 \mathrm{ml}$

Paraformaldehyde solution $4 \%$

$40 \mathrm{~g}$ of paraformaldehyde in $0.1 \mathrm{M} \mathrm{PB}$; total volume $1 \mathrm{~L}$

Normal saline solution

$9 \mathrm{~g}$ of $\mathrm{NaCl}$ in DDW; total volume 1L

Avertin

$4.5 \mathrm{~mL}$ normal saline, $400 \mu \mathrm{l}$ ethanol, $100 \mu \mathrm{l}$ of avertin stock solution.

Avertin stock solution

5g 2,2,2- Tribromoethanol in 5 mL 2 methyl-2-butanol.

In vitro electrophysiology

\section{0x ACSF stock solution}

$72.47 \mathrm{~g}$ of $\mathrm{NaCl}, 2.01 \mathrm{~g}$ of $\mathrm{KCl}, 21.84 \mathrm{~g}$ of $\mathrm{NaHCO}_{3}, 1.725 \mathrm{~g}$ of $\mathrm{NaH}_{2} \mathrm{PO}_{4}\left(1 \mathrm{H}_{2} \mathrm{O}\right)$ in DDW; total volume $1 \mathrm{~L}$

Sucrose solution 


\section{MATERIALS AND METHODS}

$6 \mathrm{mM} \mathrm{MgCl}_{2}, 0.1 \mathrm{mM} \mathrm{CaCl}_{2}, 8.55 \mathrm{~g}$ sucrose, $0.225 \mathrm{~g}$ glucose, $0.284 \mathrm{~g}$ of kynurenic acid in ACSF; total volume $500 \mathrm{~mL}$

\section{Internal solution (patch pipette solution)}

$110 \mathrm{mM} \mathrm{CsCl,} \mathrm{30mM} \mathrm{K-gluconate,} \mathrm{1.1mM} \mathrm{EGTA,} 10 \mathrm{mM}$ HEPES, $0.1 \mathrm{mM} \mathrm{CaCl}_{2}, 4 \mathrm{mM}$ Mg-ATP, 0.3 mM Na-GTP and 4mM N-(2,6-Dimethylphenylcarbamoylmethyl) triethylammonium bromide (QX-314; Tocris-Cookson, Ellisville, MO); pH = 7.3 (adjusted with $\mathrm{CsOH}, 280 \mathrm{mOsm})$.

\section{External solution (Slice incubation solution)}

$1.3 \mathrm{mM} \mathrm{MgCl}_{2}, 2 \mathrm{mM} \mathrm{CaCl}_{2}, 2.51 \mathrm{~g}$ glucose, $0.297 \mathrm{~g}$ ascorbic acid in ACSF; total volume 750 $\mathrm{mL}$; osmolarity $\approx 320 \mathrm{mOsm}$.

\section{Stereotaxic surgery}

Sterile normal saline $(0.9 \% \mathrm{NaCl}$ in DDW).

\subsection{Materials}

Table 2.8| List of materials.

$\begin{array}{lll}\text { Immunohistochemistry } & \text { Electrophysiology } & \text { Stereotaxic surgery }\end{array}$

\begin{tabular}{|c|c|c|c|c|c|}
\hline Name & Company & Name & Company & Name & Company \\
\hline 2,2,2- Tribromoethanol & $\begin{array}{l}\text { Alfa } \\
\text { Aesar }\end{array}$ & $\begin{array}{l}\text { D-(+)- Glucose } \\
\text { monohydrate }\end{array}$ & $\begin{array}{l}\text { Sigma- } \\
\text { Aldrich }\end{array}$ & $\begin{array}{l}\text { Metamizol } \\
\text { e }\end{array}$ & "Ratiopharm \\
\hline 2 methyl-2-butanol & $\begin{array}{l}\text { Sigma- } \\
\text { Aldrich }\end{array}$ & Kynurenic acid & $\begin{array}{l}\text { Sigma- } \\
\text { Aldrich }\end{array}$ & Caprofen & Pfizer \\
\hline Isofluran & Zoetis & $\begin{array}{l}\text { Magnesium } \\
\text { chloride } \\
\text { hexahydrate }\end{array}$ & $\begin{array}{l}\text { Merk } \\
\text { Millipore }\end{array}$ & Visidic & $\begin{array}{l}\text { Bausch } \\
\text { Lomb }\end{array}$ \\
\hline Paraformaldehyde & Serva & $\begin{array}{l}\text { L-(+)- Ascorbic } \\
\text { acid }\end{array}$ & $\begin{array}{l}\text { Merk } \\
\text { Millipore }\end{array}$ & Iodine & Animedica \\
\hline Sodium chloride & $\begin{array}{l}\text { Merk } \\
\text { Millipore }\end{array}$ & Tetrodotoxin & $\begin{array}{l}\text { Tocris } \\
\text { Bioscience }\end{array}$ & RetroBeads & $\begin{array}{l}\text { Lumafluor } \\
\text { Inc. }\end{array}$ \\
\hline
\end{tabular}


MATERIALS AND METHODS

\begin{tabular}{|c|c|c|c|}
\hline $\begin{array}{l}\text { di-Sodium hydrogen }{ }^{2} \\
\text { phosphate dihydrate }\end{array}$ & $\begin{array}{l}\text { Merk } \\
\text { Millipore }\end{array}$ & $\begin{array}{l}\text { 6-cyano-7- } \\
\text { nitroquinoxaline- } \\
\text { 2,3-dione }\end{array}$ & $\begin{array}{l}\text { Tocris } \\
\text { Bioscience }\end{array}$ \\
\hline $\begin{array}{l}\text { Sodium dihydrogen } \\
\text { phosphate monohydrate }\end{array}$ & $\begin{array}{l}\text { Merk } \\
\text { Millipore }\end{array}$ & $\begin{array}{l}((R S)-3-(2- \\
\text { Carboxypiperazin- } \\
\text { 4-yl)-propyl-1- } \\
\text { phosphonic acid) }\end{array}$ & Biolab \\
\hline Potassium chloride & $\begin{array}{l}\text { Merk } \\
\text { Millipore }\end{array}$ & $\begin{array}{l}\text { Potassium } \\
\text { gluconate }\end{array}$ & $\begin{array}{l}\text { Sigma- } \\
\text { Aldrich }\end{array}$ \\
\hline Calcium chloride & $\begin{array}{l}\text { Merk } \\
\text { Millipore }\end{array}$ & $\mathrm{CsCl}$ & $\begin{array}{l}\text { Sigma- } \\
\text { Aldrich }\end{array}$ \\
\hline Sucrose & $\begin{array}{l}\text { Sigma- } \\
\text { Aldrich }\end{array}$ & EGTA & $\begin{array}{l}\text { Sigma- } \\
\text { Aldrich }\end{array}$ \\
\hline Glucose & $\begin{array}{l}\text { Sigma- } \\
\text { Aldrich }\end{array}$ & HEPES & $\begin{array}{l}\text { Sigma- } \\
\text { Aldrich }\end{array}$ \\
\hline $\begin{array}{l}\text { Potassium dihydrogen } \\
\text { phosphate }\end{array}$ & $\begin{array}{l}\text { Merk } \\
\text { Millipore }\end{array}$ & NaGTP & $\begin{array}{l}\text { Sigma- } \\
\text { Aldrich }\end{array}$ \\
\hline Immersion oil & Zeiss & $\begin{array}{l}\text { 2,6- } \\
\text { (Dimethylphenyl } \\
\text { carbamoylmethyl) } \\
\text { triethylammonium } \\
\text { bromide }\end{array}$ & $\begin{array}{l}\text { Tocris- } \\
\text { Cookson }\end{array}$ \\
\hline Triton $\mathrm{X}-100$ & Roche & \multicolumn{2}{|c|}{ In vivo electrophysiology } \\
\hline Albumin bovine & Biomol & Name & Company \\
\hline Aqua poly/mount & $\begin{array}{l}\text { Polyscien } \\
\text { ces }\end{array}$ & "Baytril & $\overline{\text { Bayer }}$ \\
\hline \multicolumn{4}{|l|}{ Goat serum } \\
\hline Donkey serum & $\begin{array}{l}\text { Sigma- } \\
\text { Aldrich }\end{array}$ & & \\
\hline Ethanol & $\begin{array}{l}\text { Sigma- } \\
\text { Aldrich }\end{array}$ & & \\
\hline 2-Methylbutan & Roth & & \\
\hline
\end{tabular}

Table 2.8 continued 
MATERIALS AND METHODS

\subsection{Equipment}

Table 2.9| List of equipment

$\begin{array}{lll}\text { Immunohistochemistry } & \text { Electrophysiology } & \text { Stereotaxic surgery }\end{array}$

\begin{tabular}{|c|c|c|c|c|c|}
\hline Name & Company & Name & Company & Name & Company \\
\hline Cryostat Leica & Leica & Vibrotome & Leica & Stereotaxic & World precision \\
\hline CM3050S & Biosystems & & Biosystems & $\begin{array}{l}\text { frame } \\
502300 \\
\text { series }\end{array}$ & instruments \\
\hline Leica SP2 & $\begin{array}{l}\text { Leica } \\
\text { Biosystems }\end{array}$ & $\begin{array}{l}\text { EPC10 } \\
\text { Amplifier }\end{array}$ & HEKA & $\begin{array}{l}\text { Hamilton } \\
\text { syringe } \\
7000 \text { series }\end{array}$ & Hamilton \\
\hline Leica MZ16 & $\begin{array}{l}\text { Leica } \\
\text { Biosystems }\end{array}$ & $\begin{array}{l}\text { Microscope } \\
\text { UCMAD3 }\end{array}$ & Olympus & Drill & $\begin{array}{l}\text { World precision } \\
\text { instruments }\end{array}$ \\
\hline $\begin{array}{l}\text { Aqua- } \\
\text { Poly/Mount }\end{array}$ & Polysciences & $\begin{array}{l}\text { Boroscillate } \\
\text { glass tubes, } \\
\text { GB150F-8P }\end{array}$ & $\begin{array}{l}\text { Science } \\
\text { products }\end{array}$ & Drill bits & \\
\hline
\end{tabular}

In vivo electrophysiology

\begin{tabular}{|c|c|}
\hline Name & Company \\
\hline Steriotaxic frame & Kopf Inc. \\
\hline $\begin{array}{l}\text { Rectal probe } \\
\text { ATC } 1000\end{array}$ & WPI \\
\hline Tungsten wires & $\begin{array}{l}\text { California Fine } \\
\text { Wire }\end{array}$ \\
\hline Polymide tubes & $\begin{array}{l}\text { Science } \\
\text { Products }\end{array}$ \\
\hline Connector & Omnetics \\
\hline Amplifier HS-18 & Neuralynx \\
\hline
\end{tabular}


MATERIALS AND METHODS

Acquisition Neuralynx

board Digital

Lynx 4SX

\subsection{Software}

Table 2.10| List of software.

Immunohistochemistry Electrophysiology In vivo Electrophysiology

\begin{tabular}{llllll}
\hline Name & Company & Name & Company & Name & Company \\
\hline \hline Imaris & Bitplane & Igor Pro & Wave Metrics & MATLAB & Mathworks \\
ImageJ & NIH & Patchmaster & HEKA & $\begin{array}{l}\text { Cheetah Data } \\
\text { Acquisition } \\
\text { System }\end{array}$ & Neuralynx \\
& & & & & \\
& & & & &
\end{tabular}


RESULTS

\section{RESULTS}

Chapter 1- Role of Neuroligin 2 in Inhibitory Synaptic Function and Anxiety- Associated Neuronal Activation of Amygdala.

Out of many genetic causes of neuropsychiatric conditions, mutations in genes regulating synaptic function are increasingly linked to pathophysiology of disorders such as autism and anxiety (Lüthi \& Lüscher 2014; Thomas C Südhof 2008). One prominent example is the family of Neuroligins adhesion proteins that maintain the function of synapses- in which more than 30 mutations were identified in autistic patients (Jamain et al. 2003). Previous studies described the function of Neuroligin 3 and Neuroligin 4 in specific synaptic networks within neural circuits underlying behavioral phenotypes associated with autism symptoms (Rothwell et al. 2014; Földy et al. 2013; Hammer et al. 2015), thus shedding light on molecular mechanisms of autism.

Neuroligin 2, whose loss of function mutation was linked to schizophrenia and anxiety in humans (Sun et al. 2011; Parente et al. 2016) is emerging as a one of key regulators of excitation/inhibition balance in neural circuits underlying psychiatrically relevant behaviors, such as social behavior (Kohl et al. 2013; Kohl et al. 2015; Liang et al. 2015). The robust anxiety phenotype in Nlgn2 KO mice indicates that Nlgn 2 must also play a central role in the neural circuitry underlying anxiety behaviors, but to date, virtually nothing is known about the function of Nlgn2 in these circuits. To address this problem, I investigated the molecular, cellular and physiological consequences of Nlgn2 KO mice in the amygdala, a brain region that has been prominently implicated in fear and anxiety behaviors (LeDoux 2003). For that, I characterized the behavior of Nlgn2 KOs using anxiety assay and then employed a "top- down" approach by first identifying a brain region and then a subpopulation of synapses and finally a specific neuron type affected by deletion of Nlgn2. Taken together, my findings support a model according to which reduced perisomatic inhibition at connections between Parvalbumin positive interneurons and projection neurons in BA of Nlgn2 KO mice results in specific overactivation of these projection neurons under anxiogenic conditions. Thus, this study provides an important insight into possible molecular mechanisms by which mutations in Nlgn2 contribute to pathophysiology of anxiety and comorbid disorders. 


\section{RESULTS}

\subsubsection{Nlgn2 KO Causes a Robust Anxiety Phenotype}

Nlgn2 KO mice have previously been reported to display increased anxiety-related behaviors (Blundell et al. 2009) but the extent of this anxiety phenotype has been discussed controversially (Blundell et al., 2009, Wohr et al. 2013) To address this issue and to characterize the anxiety phenotype under our experimental conditions, I performed a battery of anxiety tests on Nlgn2 KO, Nlgn2 Het and WT littermates, including the elevated plus maze (EPM), the open field test (OFT) and the light/dark exploration test (LDT). Nlgn2 KO mice showed a robust anxiety phenotype in all these tests (Figure 3.1.1, Figure 3.1.2), spending significantly less time and making fewer entries into the open arms of the EPM, the center of the OFT, and the light zone of the LDT (Figure 3.1.1A-C, Figure 3.1.2A). Similarly, the distance traveled in the open arms of the EPM, the center of the OFT, and the light zone of the LDT was significantly reduced in Nlgn2 KO mice (Figure 3.1.1D-F). Nlgn2 Het mice exhibited a mild anxiety phenotype only in the EPM (Figure 3.1.1A, 3.1.1D; Figure 3.1.2A, B), with no significant differences from WT mice observed in the other two anxiety tests (Figure 3.1.1B, C, E, F; Figure 3.1.2). Given that Nlgn2 Het mice showed only a very mild behavioral phenotype, the subsequent molecular, cellular, and electrophysiological characterization was restricted to the comparison of WT and Nlgn2 KO mice.

\subsubsection{Nlgn2 KO Causes Reduced Locomotor Activity and Enhanced Freezing Specifically Under Anxiogenic Conditions}

Interestingly, the total distance traveled by Nlgn2 $\mathrm{KO}$ mice in the EPM and OFT was also significantly reduced as compared to WT and Het mice (Figure 3.1.2 B, C; this analysis could not be performed for the LDT for technical reasons, since the experimental setup did not permit me to measure activity levels in the dark zone). To determine whether this reduction in locomotor activity may confound the anxiety phenotype in the KO mice, I performed three additional analyses. First, I normalized the distance traveled in the open arms of the EPM and the center of the OFT to the total distance traveled in the EPM and OFT, respectively (Figure 3.1.2D, E). This analysis revealed that the anxiety phenotype is still highly significant even after correcting for overall activity levels. Second, to rule out a primary locomotor impairment, I monitored activity under basal, nonanxiogenic conditions in a home cage setting. Under these 

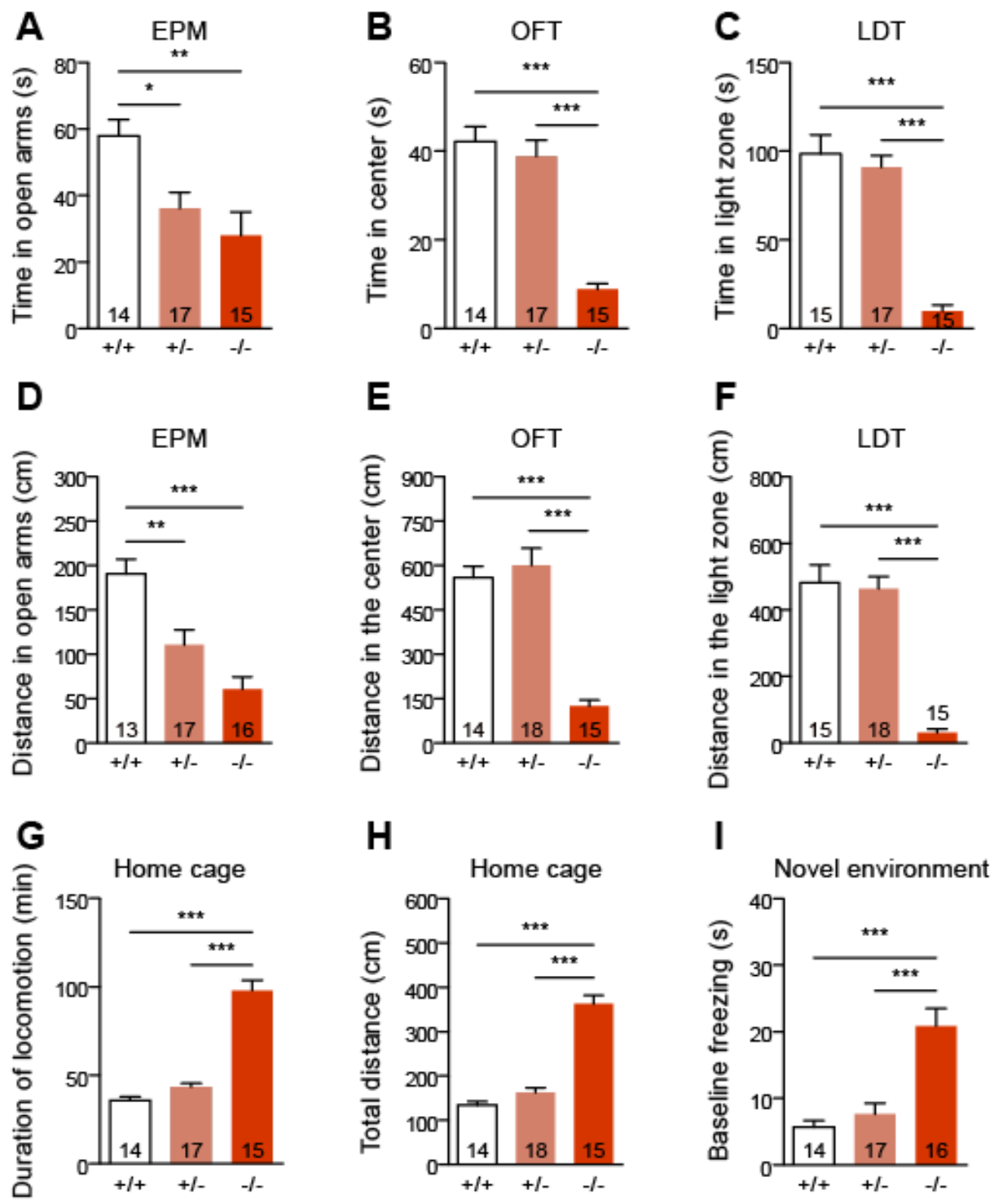

Figure 3.1.1| Anxiety phenotype and locomotor activity of Nlgn2 KO mice.

A-C. Time spent by WT, Nlgn2 Het, and Nlgn2 KO mice in the open arms of elevated plus maze (A), center of the open field $(\mathbf{B})$, and light zone of light dark box $(\mathbf{C})$. One-way ANOVA for genotype: (A) $\mathrm{F}_{2,43}=7.07, \mathrm{p}<0.01$, (B) $\mathrm{F}_{2,43}=37.33, \mathrm{p}<0.0001$, (C) $\mathrm{F}_{2,44}=42.73, \mathrm{p}<0.0001$.

D-F. Distance traveled in the open arms of elevated plus maze $(\mathbf{D})$, center of the open field $(\mathbf{E})$, and light zone of light dark box (F). One-way ANOVA for genotype: (D) $F_{2,43}=15.83$, p $<0.0001$; (E) $F_{2,44}=$ 35.43 , p <0.0001; (F) $\mathrm{F}_{2,45}=44.62$, $\mathrm{p}<0.0001$.

G-H. Duration of locomotor activity $(\mathbf{G})$ and distance traveled $(\mathbf{H})$ in a home cage setting during $15 \mathrm{~h}$ of recording. One-way ANOVA for genotype: $(\mathrm{G}) \mathrm{F}_{2,43}=79.75$, $\mathrm{p}<0.0001 ;(\mathrm{H}), \mathrm{F}_{2,46}=61.95, \mathrm{p}<0.0001$.

I. Duration of freezing in a novel environment. One-way ANOVA for genotype: (I) $F_{2,44}=17.98$, $p<$ 0.0001. Post hoc Tukey's test: $* \mathrm{p}<0.05, * * \mathrm{p}<0.01$, *** $\mathrm{p}<0.001 . n=14-18$ for each genotype. All bars represent mean + SEM. 

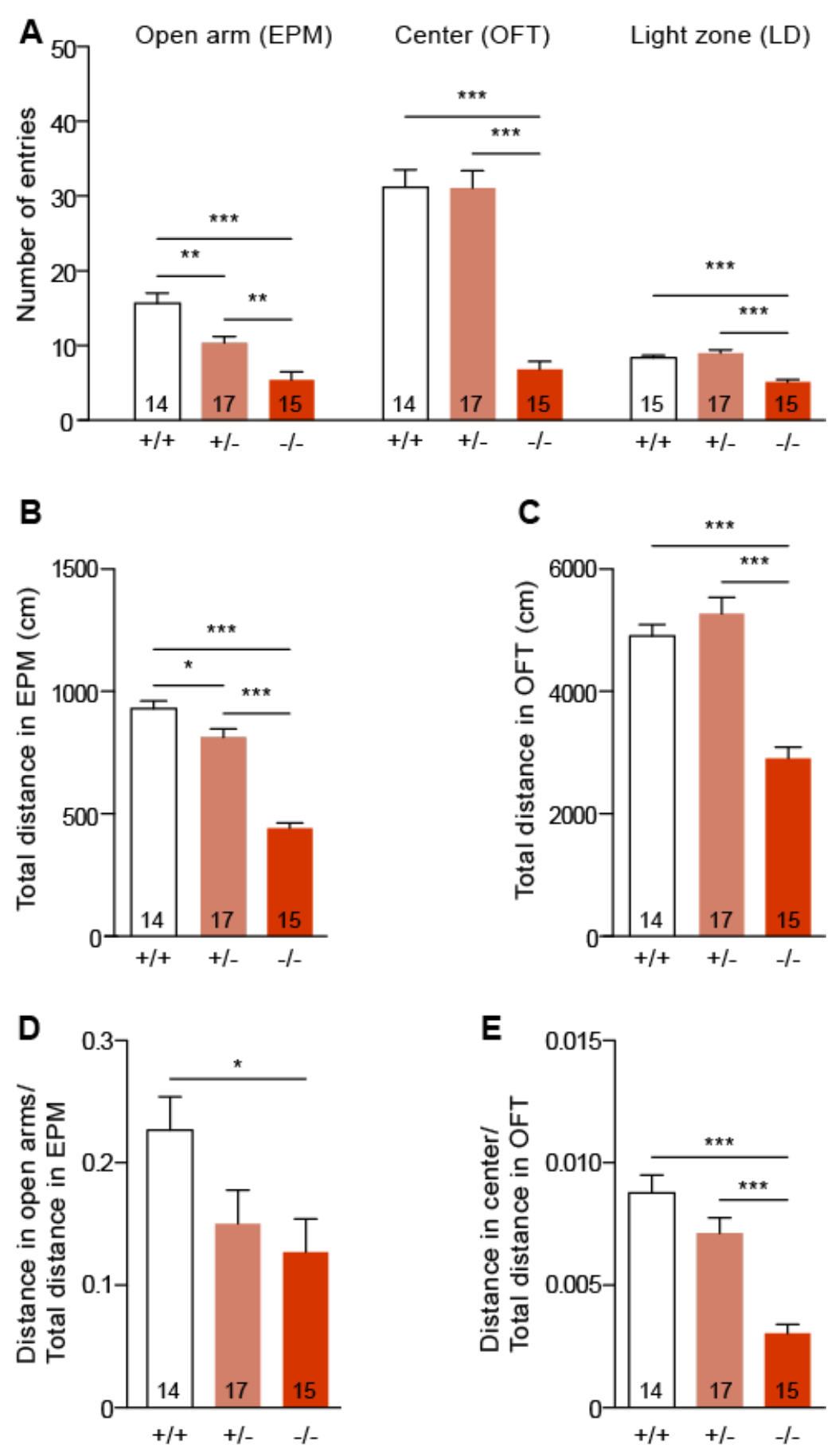

Figure 3.1.2 Horizontal activity of Nlgn2 KO mice during anxiety testing.

A. Number of entries to open arms of elevated plus maze, center of the open field and light zone of light dark box. One-way ANOVA: (EPM entries) $\mathrm{F}_{2,43}=21.14$, $\mathrm{p}<0.0001$; (OFT entries) $\mathrm{F}_{2,45}=44.80, \mathrm{p}<$ 0.0001; (LDT entries) $\mathrm{F}_{2,46}=28.51, \mathrm{p}<0.0001$.

B-C. Total distance traveled in elevated plus maze $(\mathbf{B})$ and open field chamber $(\mathbf{C})$. One-way ANOVA for genotype: (B) $\mathrm{F}_{2,45}=67.41, \mathrm{p}<0.0001 ;(\mathrm{C}) \mathrm{F}_{2,45}=31.25, \mathrm{p}<0.0001$. 


\section{RESULTS}

D, E. Ratio between distance traveled in the open arms of EPM (D) and center of the OFT (E) and respective total distance traveled in EPM or OFT. One- way ANOVA (EPM ratio): F2, $45=3.32, \mathrm{p}=0.045$; (OFT ratio) F2, $45=24.47, \mathrm{p}<0.0001$. All bars represent mean $+\mathrm{SEM}, * * \mathrm{p}<0.01$, *** $\mathrm{p}<0.001 . n=14-18$ per genotype.

conditions, Nlgn2 KO mice surprisingly showed an increase in locomotion (Figure 3.1.1G, H) and a corresponding decrease in time spent immobile (Figure 3.1.3A), while additional aspects of home cage activity did not differ (Figure 3.1.3). Third, based on the empirical observation that Nlgn2 KO mice showed increased freezing during the anxiety tests, I also directly measured freezing behavior in a novel anxiogenic environment. Nlgn2 KO mice spent significantly more time freezing following first exposure to a novel chamber (Figure 3.1.1I). This increase in freezing likely contributes to the reduced exploratory activity, which is therefore one of the manifestations of the robust anxiety phenotype in Nlgn2 KO mice.

\subsubsection{Enhanced cFOS Activation in Basal Amygdala Neurons of NIgn2 KO Mice Upon Exposure to an Anxiogenic Environment}

To elucidate the cellular and molecular basis for the anxiety phenotype in Nlgn2 KO mice, I investigated whether differential activation of neurons could be observed in various brain regions associated with anxiety processing, including the amygdala and prefrontal cortex (Lüthi \& Lüscher 2014; Singewald et al. 2003). To this end, I exposed WT and Nlgn2 KO mice to an anxiogenic environment (10 min in an open field arena) and subsequently used an immunohistochemical approach to assess the expression of cFOS, a marker of neuronal activity (Sagar et al. 1988). In the amygdala (Figure 3.1.4A), I observed an increase in overall cFOS activation of basal amygdala (BA) neurons in Nlgn2 $\mathrm{KO}$ mice, which was significant in the anterior basal region (Figure 3.1.4B), but not in lateral, posterior, or ventromedial regions (Figure 3.1.4C-E). This increase was triggered by the anxiogenic situation, since cFOS expression under basal conditions did not significantly differ between WT and Nlgn2 KO mice (Figure 3.1.6). In contrast, we observed no genotype difference in cFOS immunoreactivity in two areas of prefrontal cortex, the infralimbic and prelimbic cortices (Figure 3.1.5). These findings indicate that the BA is one of the brain 
RESULTS

A

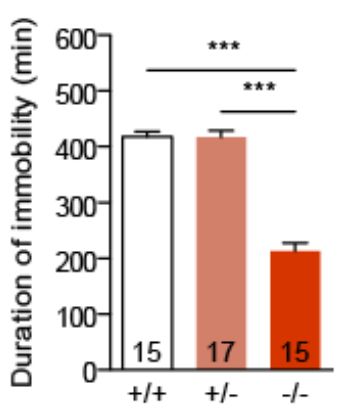

B

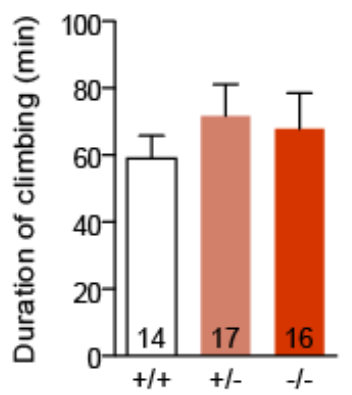

C

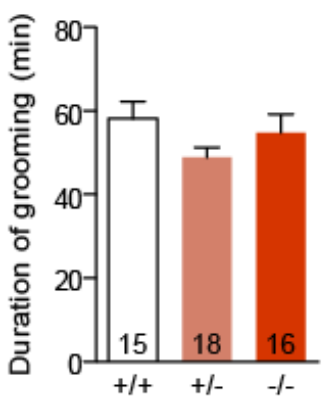

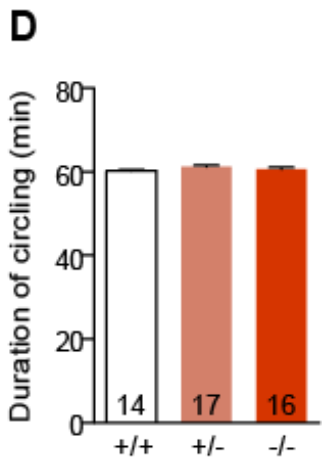

Figure 3.1.3| Activity of Nlgn2 KO mice in home cage setting.

(A-D) Duration of immobility (A), climbing (B), grooming (C), and circling behavior (D) of Nlgn2 KOs, Nlgn2 Hets, and WT mice during $15 \mathrm{~h}$ of recording. One-way ANOVA for genotype: (A) $\mathrm{F}_{2,44}=98.12, \mathrm{p}<$ 0.0001 ; (B) $\mathrm{F}_{2,44}<1$; (C) $\mathrm{F}_{2,44}<1$; (D) $\mathrm{F}_{2,44}<1$. All bars represent mean + SEM, *** $\mathrm{p}<0.001 . n=14-18$ per genotype.

regions involved in the anxiety phenotype of Nlgn2 $\mathrm{KO}$ mice, and I therefore focused our further analyses on this region.

\subsubsection{Nlgn2 is Localized to Inhibitory Synapses in the Basal Amygdala}

To investigate the molecular mechanisms underlying the increased anxiety-triggered activation of BA neurons in Nlgn2 KOs, we first assessed the expression pattern of Nlgn2 in the amygdala of WT mice. We found that Nlgn2 is highly expressed in the basal nucleus but not in the central nucleus of the amygdala (Figure 3.1.7A). To characterize which synapses contain Nlgn2 in this region we performed double labeling of Nlgn2 with PSD-95, a marker of excitatory synapses, and with gephyrin, a marker of inhibitory synapses. Our data show that Nlgn2 colocalizes with gephyrin (Figure 3.1.7B; Pearson's correlation coefficient $=0.62$ ) but not with PSD-95 (Figure 3.1.7C; Pearson's correlation coefficient $=0.01$ ), consistent with previous reports on Nlgn2 distribution in other brain regions, including retina (Hoon et al. 2009) and hippocampus (Poulopoulos et al. 2009; Varoqueaux et al. 2006). Approximately $76 \%$ of Nlgn2 puncta were colocalized with gephyrin, indicating that Nlgn2 is localized primarily to inhibitory synapses in BA. Interestingly, $78 \%$ of gephyrin puncta were also positive for Nlgn2, indicating that Nlgn2 is present at the majority of inhibitory synapses in BA. 


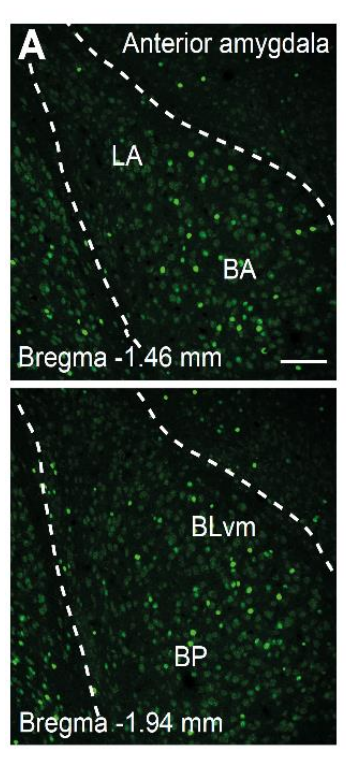

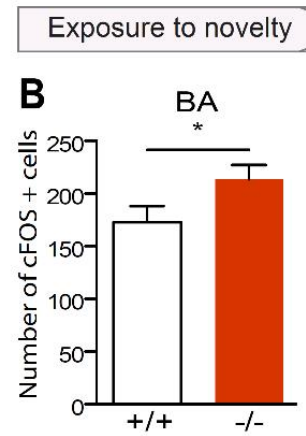

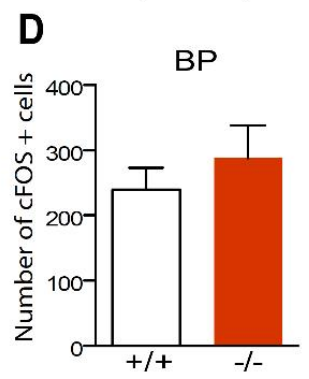

Basal amygdala
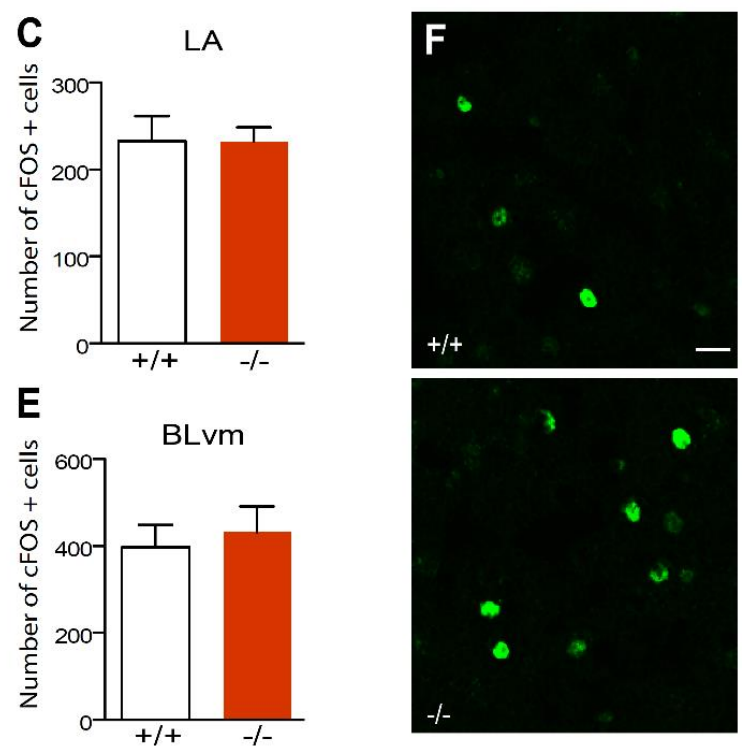

Figure 3.1.4| Anxiety-induced cFOS expression in basolateral amygdala of Nlgn2 KO mice.

A. Low magnification photomicrographs of cFOS labeled basolateral amygdala indicate the regions included in the analysis. Scale bar, $100 \mu \mathrm{m}$.

B-E. Quantification of cFOS positive cells in anterior BA (B), lateral amygdala (C), posterior BA (D), and basolateral ventromedial amygdala (E) in WT and Nlgn2 KO mice. cFOS expression was triggered by exposure to novel environment.

F. Representative photomicrographs of cFOS expression in anterior BA of WT and Nlgn2 KO mice after exposure to novel environment. Scale bar, $20 \mu \mathrm{m}$. Paired Student's t-test: $* \mathrm{p}<0.05 . n=6$ for each genotype in figures $\mathrm{B}, \mathrm{D}$ and $\mathrm{E}, n=5$ for each genotype in figure C. All bars represent means $+\mathrm{SEM}$.

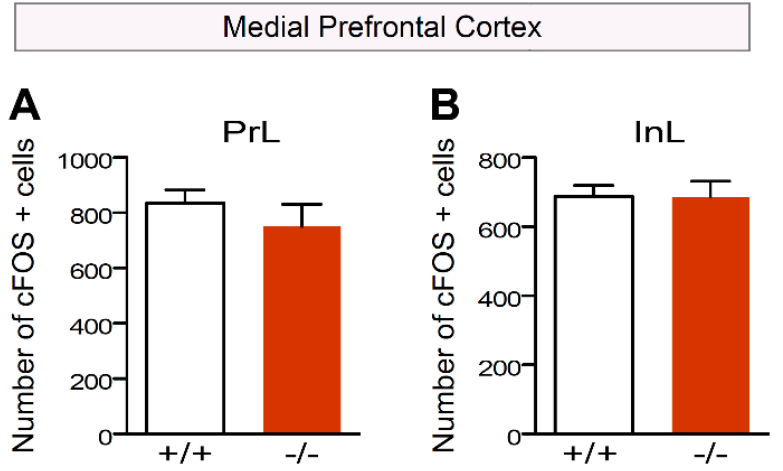

Figure 3.1.5 | Anxiety induced cFOS expression in cortical components of anxiety circuitry.

A-B. Quantification of cFOS positive cells in prelimbic (A) and infralimbic (B) regions of medial prefrontal cortex. All bars represent means + SEM. $n=5$ for each genotype. 


\section{RESULTS}
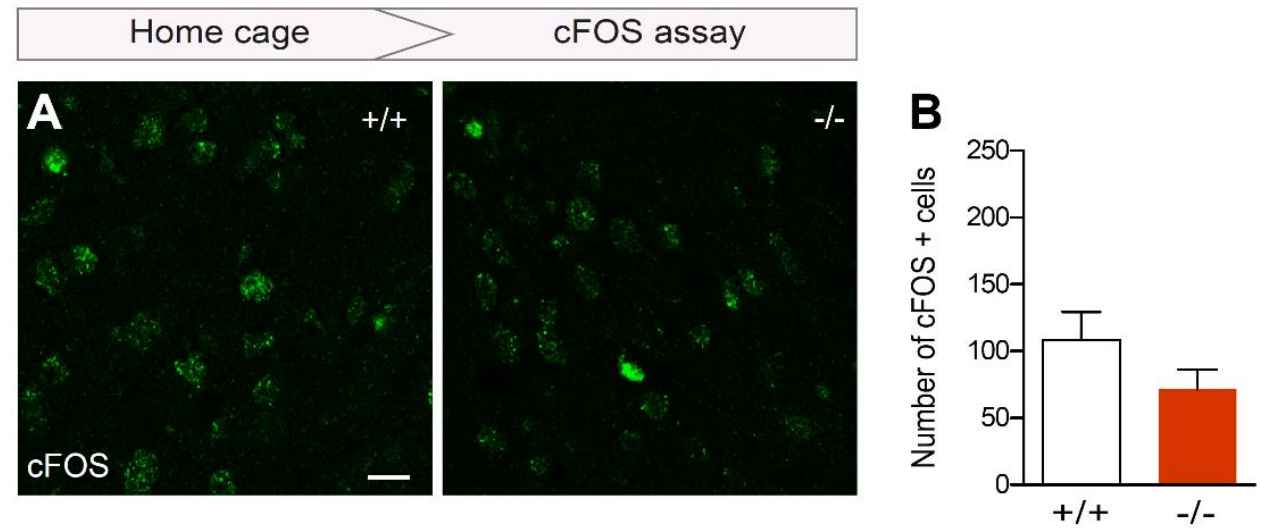

Figure 3.1.6 | Basal cFOS expression in anterior BA of WT and Nlgn2 KO mice.

A-B. Photomicrographs of cFOS expression in basal amygdala (A) and quantification of cFOS positive cells $(\mathbf{B})$ in anterior BA of mice that were taken from their home cages. All bars represent mean + SEM, $n$ $=5$.
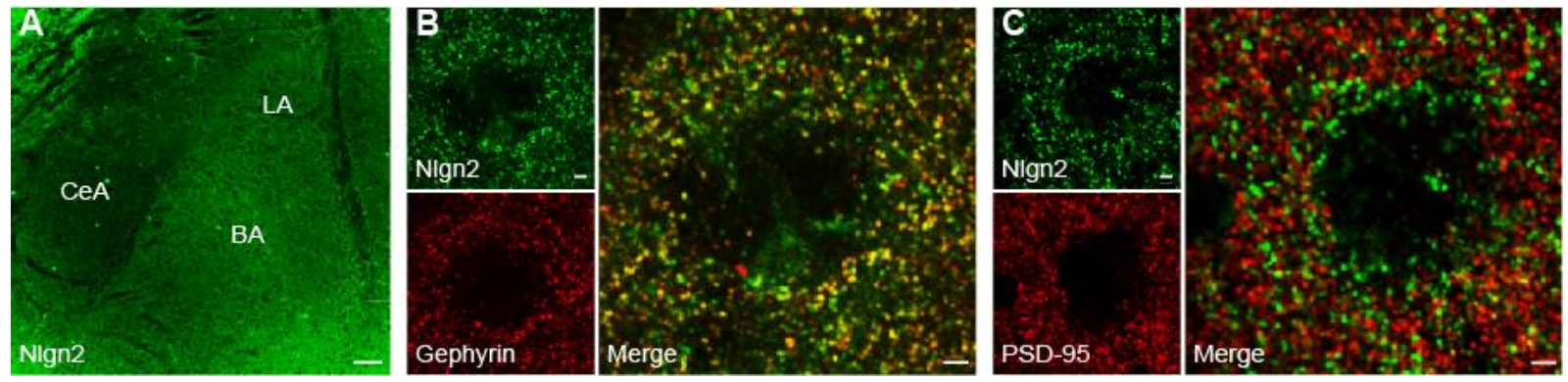

Figure 3.1.7| Nlgn2 localization in the amygdala.

A. Immunostaining shows that Nlgn2 is highly expressed in the basal and lateral nuclei of the amygdala, while its expression in the CeA is low. Scale bar, $100 \mu \mathrm{m}$.

B-C. Colocalization of Nlgn2 with gephyrin, a marker of inhibitory synapses (B), and PSD- 95, a marker of excitatory synapses $(\mathbf{C})$. Scale bar, $2 \mu \mathrm{m}$. 
RESULTS

\subsubsection{Nlgn2 KO Perturbs the Composition of Perisomatic Postsynaptic Sites in the Basal Amygdala}

Given the localization pattern of Nlgn2 at inhibitory synapses, I next investigated whether the loss of Nlgn2 affects the structure of inhibitory synapses in the BA by quantifying the number and intensity of gephyrin puncta. The overall gephyrin intensity was not altered upon Nlgn2 loss (Figure 3.1.8A-B). However, a specific analysis of perisomatic regions (Figure 3.1.8C-D) revealed a significant reduction in the number, size, and intensity of gephyrin puncta in Nlgn2 KOs (Figure 3.1.8E-G). To investigate whether this reduction is caused by a decrease in the total number of inhibitory synapses, I stained for the vesicular inhibitory amino acid transporter (VIAAT), a marker of inhibitory presynaptic terminals (Figure 3.1.9A), and found that the number and size of perisomatic VIAAT puncta are not altered upon Nlgn2 deletion (Figure 3.1.9B-C). To confirm this finding, I quantified the number of parvalbumin (PV) puncta in the BA (Figure 3.1.9D). PVpositive interneurons are the major source of perisomatic synapses in BA (Muller et al. 2006; Spampanato et al. 2011), and PV puncta therefore specifically represent perisomatic inhibitory presynaptic sites. The number and intensity of PV-positive puncta was not altered in Nlgn2 KO mice (Figure 3.1.9E-F). Taken together, these findings imply that Nlgn2 deletion does not result in a decrease in overall inhibitory synapse number in BA, but instead primarily affects the molecular composition of perisomatic inhibitory postsynaptic sites. 


\section{RESULTS}
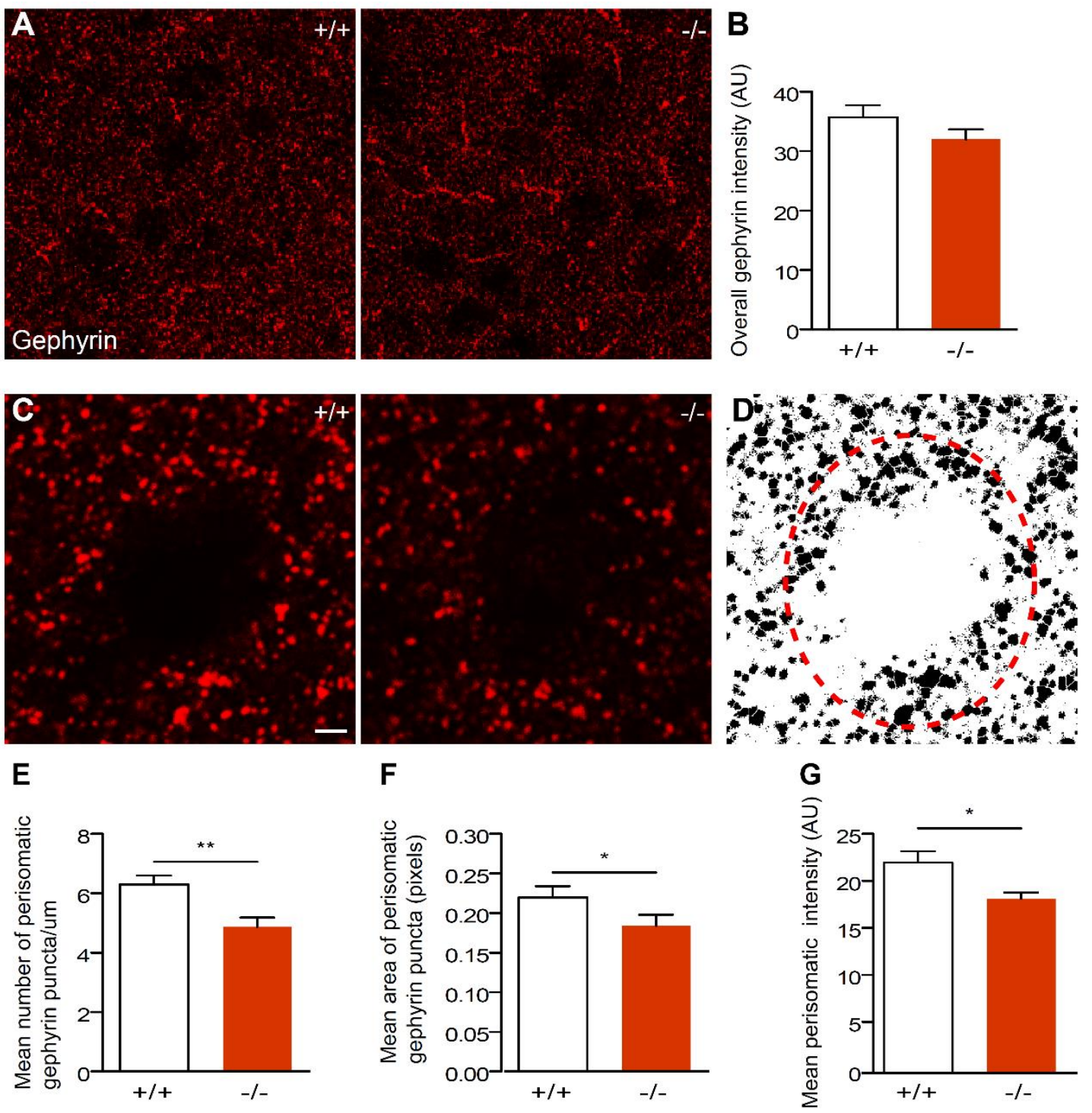

Figure 3.1.8 $\mid$ Localization and expression levels of gephyrin in BA of Nlgn2 KO mice.

A. Photomicrographs show overall gephyrin distribution in BA of WT and Nlgn2 KO mice. Scale bar, 10 $\mu \mathrm{m}$.

B. Mean fluorescence intensity of overall gephyrin immunostaining. $n=6$ for each genotype.

C-D. High magnification photomicrographs show perisomatic gephyrin localization in BA of WT and Nlgn2 KO (C). The perisomatic area is indicated on a thresholded image (D). Scale bar, $2 \mu \mathrm{m}$.

E-G. Mean number per cell perimeter $(\mathbf{E})$, mean area $(\mathbf{F})$, and mean intensity $(\mathbf{G})$ of perisomatic gephyrin puncta in BA. $n=8$ for each genotype. Paired, two-tailed Student's t-test: $* \mathrm{p}<0.05,{ }^{*} \mathrm{p}<0.01$. All bars represent mean + SEM 

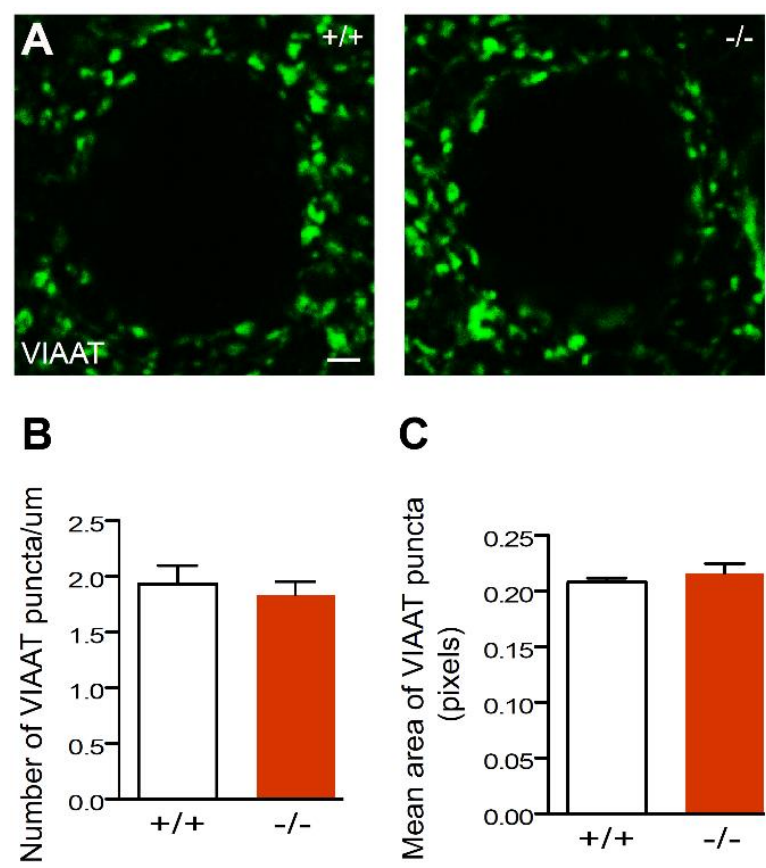

C

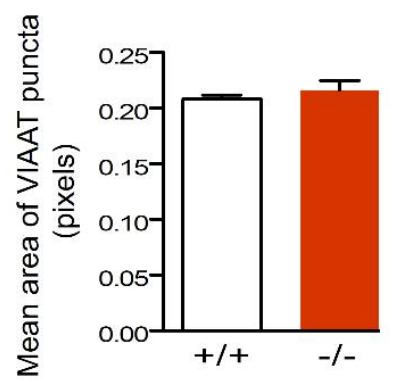

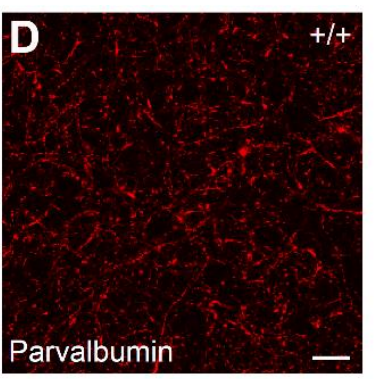

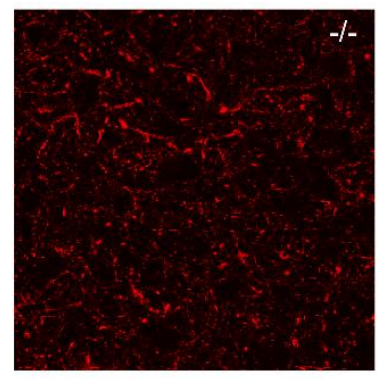

E

$\mathbf{F}$

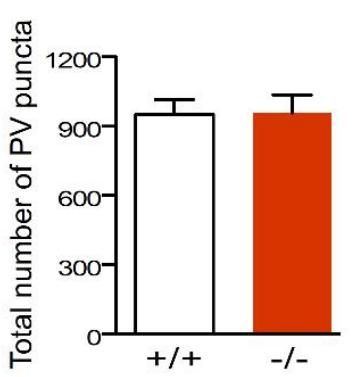

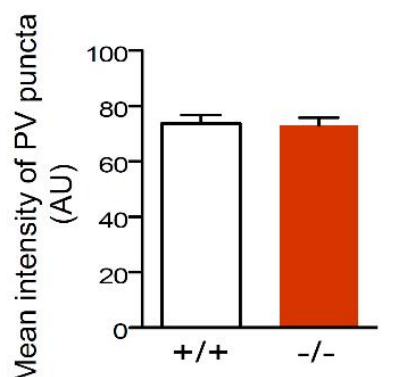

Figure 3.1.9| Localization and expression levels of presynaptic inhibitory markers in BA of Nlgn2 KO mice.

A. Photomicrographs show perisomatic VIAAT expression in BA of WT and Nlgn2 KO mice. Scale bar, $2 \mu \mathrm{m}$.

B-C. Mean number per cell perimeter $(\mathbf{B})$ and mean area $(\mathbf{C})$ of perisomatic VIAAT puncta.

D. Photomicrographs show overall localization and expression levels of PV in anterior BA of WT and Nlgn2 KO mice. Scale bar, $20 \mu \mathrm{m}$.

E-F. Total number (E) and mean intensity (F) of PV puncta in analyzed area of BA. $n=6$ for both genotypes. All bars represent mean + SEM.

\subsubsection{Nlgn2 KO Impairs Inhibitory Synaptic Transmission in the Basal Amygdala}

To assess whether deletion of Nlgn2 alters synaptic transmission, Paolo Botta (Friedrich Miescher Institute for Biomedical Research, Basel, Switzerland) measured miniature inhibitory postsynaptic currents (mIPSCs) in the BA and central amygdala (CeA, Figure 3.1.10). A pronounced reduction in mIPSC frequency but not amplitude (Figure 3.1.10 E-F) was observed in BA of Nlgn2 KO mice, while mIPSC kinetics were not significantly altered (rise time: WT $=1.40 \mathrm{~ms}, \mathrm{KO}=1.57 \mathrm{~ms}, \mathrm{p}=$ 0.13; decay time: $\mathrm{WT}=4.14 \mathrm{~ms}, \mathrm{KO}=2.87 \mathrm{~ms}, \mathrm{p}=0.09)$. In CEA, no changes were observed in mIPSC frequency or amplitude (Figure 3.1.10 G-H). 


\section{RESULTS}

A

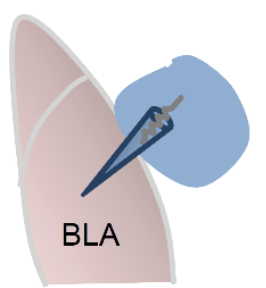

B
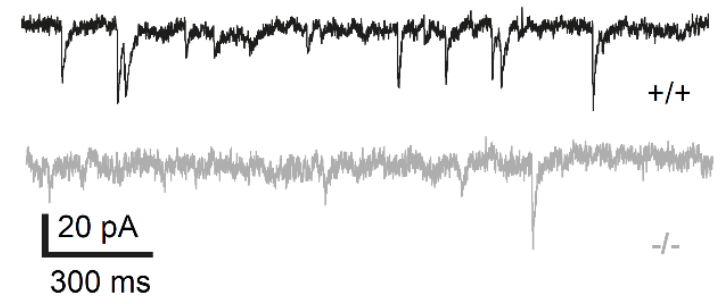

C

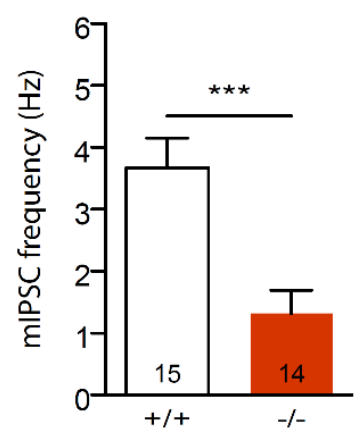

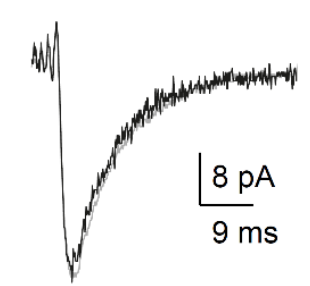

D

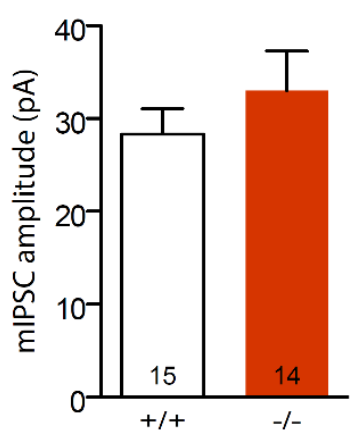

E

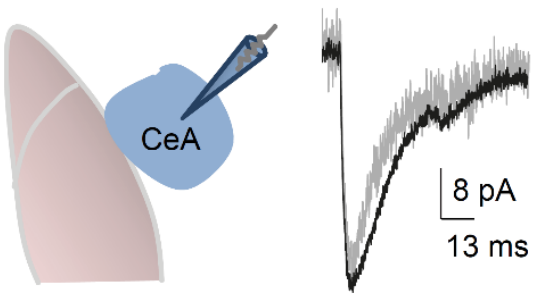

F
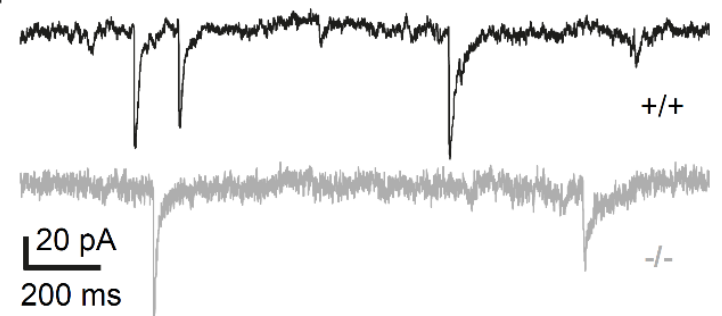

G

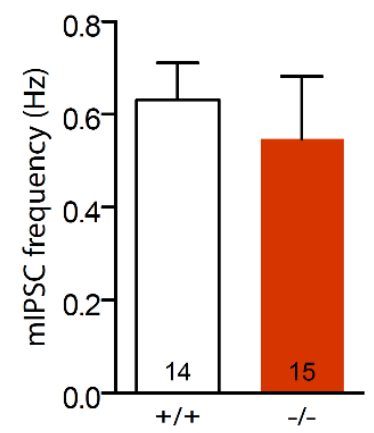

Figure 3.1.10| Effect of Nlgn2 deletion on inhibitory synaptic transmission in BA and CeA.

A-B. Schematic diagram showing the location of recordings and representative miniature IPSCs in BA (A) and CeA (B) of WT (black) and Nlgn2 KO mice (grey).

C-D. Sample traces from BA (C) and CeA (D) of WT and Nlgn2 KO mice.

E-F. Average frequency $(\mathbf{E})$ and amplitude $(\mathbf{F})$ of mIPSCs in BA of WT (black) and Nlgn2 KO mice (white).

G, H. Average frequency $(\mathbf{G})$ and amplitude $(\mathbf{H})$ of mIPSCs in CeA of WT (black) and Nlgn2 KO mice (white). $n=14-15$ for both genotypes. Unpaired, two-tailed Student's t-test: $* * * \mathrm{p}<0.001$. All bars represent mean + SEM.

* Experiments performed by Paolo Botta. 


\section{RESULTS}

\subsubsection{Nlgn2 KO has Differential Effects on Anxiety-Associated Activation of Glutamatergic and GABAergic Neurons in the Basal Amygdala}

$\mathrm{BA}$ is a cortex-like structure that contains both glutamatergic projection neurons and local inhibitory interneurons (Ehrlich et al., 2009; Sah et al., 2003), each of which play a distinct role in the acquisition and expression of fear- and anxiety-related behavioral outputs (Tye et al. 2011; Namburi et al. 2015; Wolff et al. 2014). To understand the circuitry underlying the behavioral phenotype in Nlgn2 $\mathrm{KO}$ mice, it was therefore essential to investigate the influence of Nlgn2 deletion on distinct amygdala cell types. To dissect the anxiety-induced activation pattern of several types of neurons that have been previously linked to fear behaviors (Wolff et al., 2013) I performed double labeling of $\mathrm{cFOS}$ and CaMKII (as a marker for glutamatergic projection neurons), PV, or somatostatin (SOM) in WT and Nlgn2 KO animals that had been exposed to anxiogenic conditions. I quantified both the total number and the cFOS-positive percentage of each type of neuron. I found that Nlgn2 $\mathrm{KO}$ mice exhibit enhanced activation of projection neurons in BA (Figure 3.1.11A, E), with no change in the total number of projection neurons (Figure 3.1.11D). In contrast, the total number of PV-positive cells was significantly increased in Nlgn2 KO mice (Figure 3.1.11B, F), without a significant change in the percentage of PV-positive cells that showed cFOS immunoreactivity (Figure 3.1.11G). Nlgn2 deletion did not have an effect on SOM-positive interneurons, as both their total number and their cFOS-positive fraction were unaltered in Nlgn2 KO mice (Figure 3.1.11C, H, I). These data indicate that Nlgn2 deletion impacts specific types of inhibitory synapses in BA in a manner that leads to an increased anxiety phenotype of the KO mice. In addition, the enhanced PV immunoreactivity indicates a perturbed development, maintenance, or homeostasis of this inhibitory neuronal network in Nlgn2 KO mice.

\subsubsection{Summary and Conclusions}

Using a combination of behavior assessment and cFOS activation assay, I show that Nlgn2 deletion in mice leads to robust anxiety, which is accompanied by enhanced cFOS expression of anterior basal amygdala. In search for molecular mechanism of this activity upregulation I use quantitative morphological analysis and electrophysiology and show that Nlgn2 is localized exclusively at 

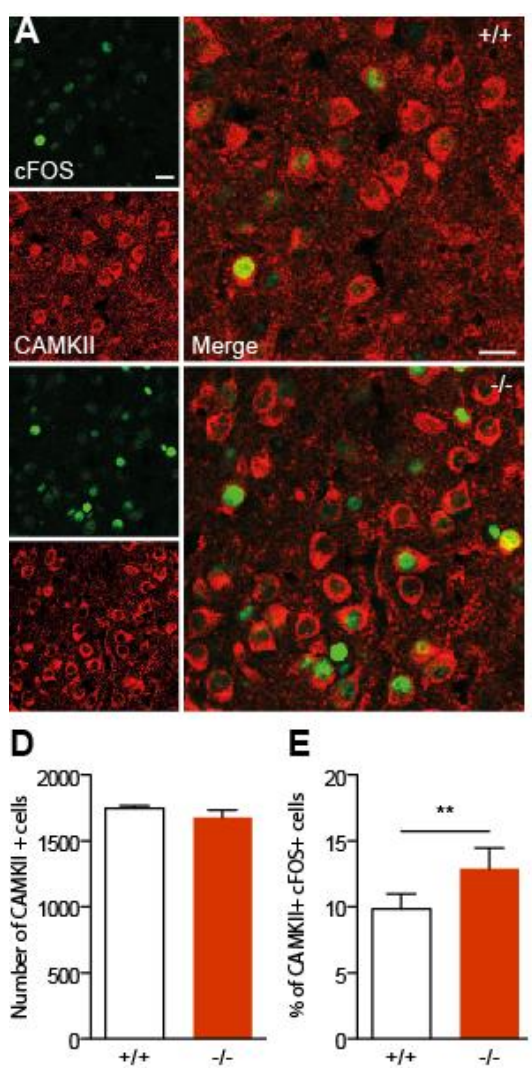
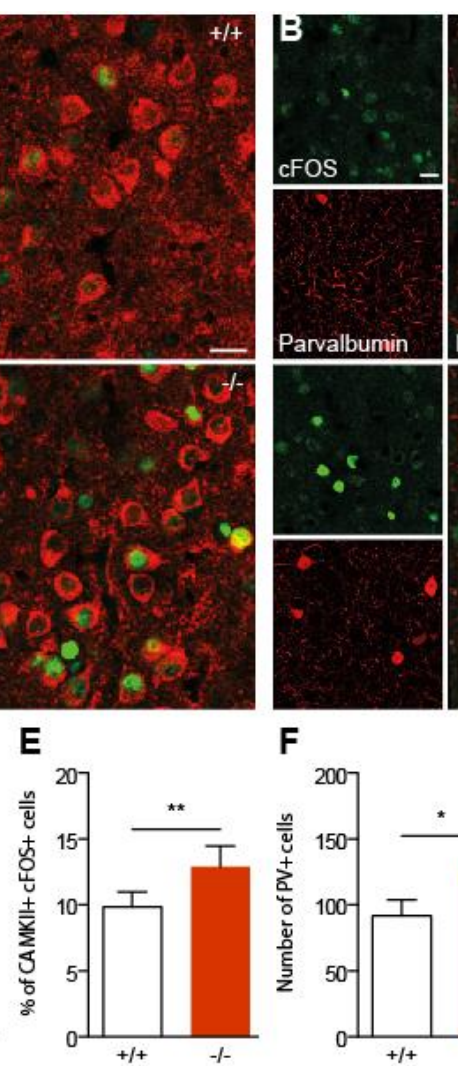
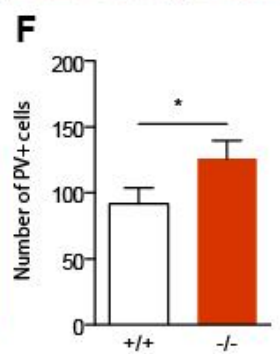

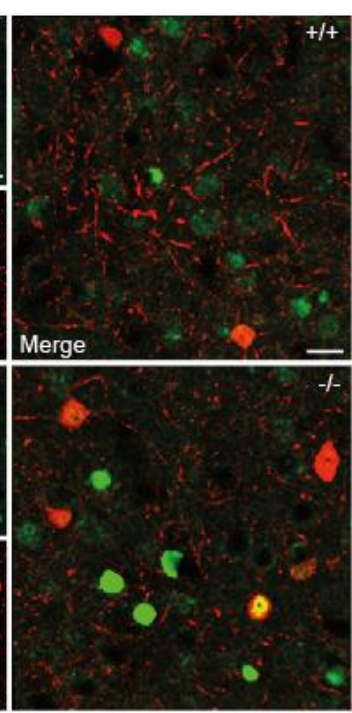

G

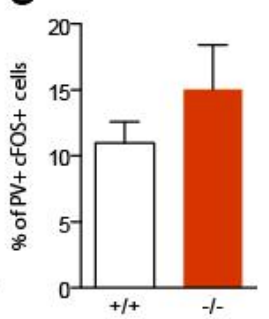

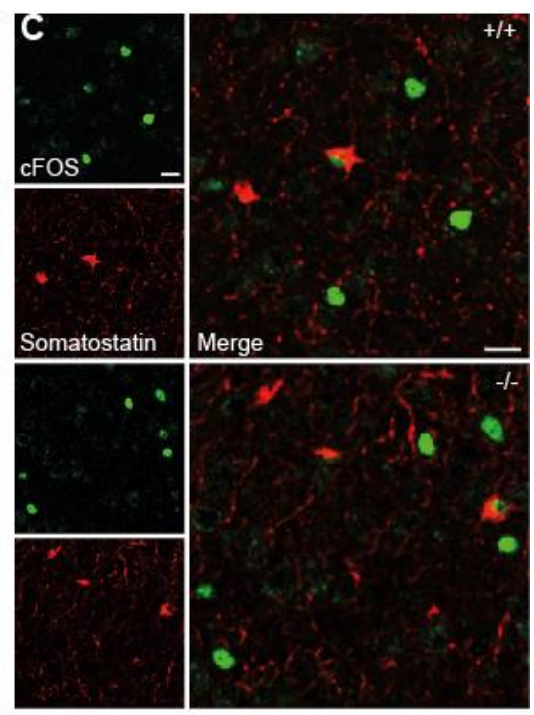

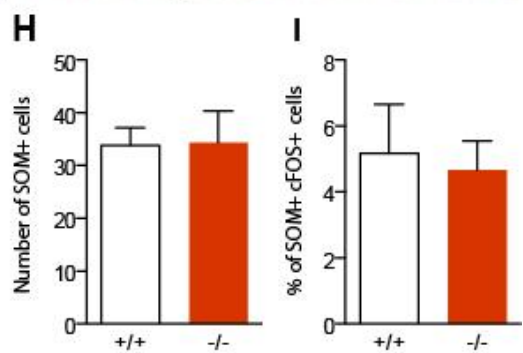

Figure 3.1.11| Effect of Nlgn2 deletion on cellular components of the anxiety circuitry in BA. A-C. Photomicrographs of anterior BA in WT mice and Nlgn2 KO mice following exposure to stress. The slices were double immunolabeled for cFOS and CaMKII (A), cFOS and PV (B), or cFOS and SOM (C). Scale bar, $50 \mu \mathrm{m}$.

D-I. Number of CaMKII (D), PV (F), and SOM (H) positive cells and percentage of CaMKII (E), PV (G), and SOM (I) positive cells that express cFOS following exposure to stress. For each genotype, $n=7$ for CaMKII/cFOS analysis, $n=6$ for analysis of inhibitory markers. Paired Student's t-test: $* \mathrm{p}<0.05$, ** $\mathrm{p}<$ 0.01. All bars represent means + SEM.

inhibitory synapses and that Nlgn2 deletion does not affect the total number of inhibitory synapses but leads to alterations in the structure and function of perisomatic inhibitory synapses in the basal amygdala. By dissecting the cFOS expression pattern to distinct types of neurons, I show that CAMKII- positive glutamatergic projection neurons show increased induction of cFOS in Nlgn2 KO mice, while inhibitory activation of inhibitory Parvalbumin and Somatostatin neurons remains unaltered. 


\section{RESULTS}

Taken together my findings suggest that deletion of Neuroligin2 specifically affects inhibitory transmission at the soma of glutamatergic neurons in basal amygdala, thus upregulating the excitatory output of basal amygdala under anxiogenic conditions. As enhanced output of basal amygdala leads to anxiety- like behavior in mice (Tye et al. 2011), my findings offer a plausible molecular mechanism of increased anxiety- like behavior of Nlgn2 KOs. Further studies will be needed to elucidate the role of Nlgn2 in downstream components of anxiety processing circuitry that were not addressed here (such as central amygdala) and find possible synaptic partners that compensate for lack of Nlgn2 at synapses that appear unaffected by Nlgn2 deletion. These studies may provide essential insights into underlying molecular mechanisms and potential drug targets for the treatment of anxiety and co morbid disorders. 


\section{RESULTS}

\section{Chapter 2 - Role of IgSF9b in Regulation of Anxiety Phenotype of Nlgn2 KO}

One of the major bottlenecks in characterizing the molecular basis of neuropsychiatric disorders is the complex relationship between genetic risk factors and pathological behaviors. Various mutations may lead to similar behavioral abnormalities despite producing distinct molecular deficits. On the other hand, a variety of pathological phenotypes with variable severity may all be linked to the same mutation (Zoghbi 2003; Bourgeron 2016). It is therefore clear that the contribution of single gene mutations to pathophysiology cannot be inferred from their impact on protein function, but rather from their collective impact on neural pathways that regulate behavior.

One such pathway linking several mutations to anxiety-related phenotypes is the inhibitory synapse in basal amygdala (Vislay et al. 2013; Müller et al. 2015). Consistent with this notion, my previous findings show that Nlgn2 $\mathrm{KO}$ produces molecular and functional deficits of inhibitory synapses in basal amygdala and increases anxiety- like behavior in mice. However, to fully characterize the role of $\mathrm{Nlgn} 2$ in anxiety, it is necessary to understand the synaptic interactions that shape the effect of Nlgn2 on anxiety related behavior.

One molecule that has been reported to act in a complex with Nlgn2 is the newly identified cell adhesion molecule IgSF9b. In cell cultures, Nlgn2 and IgSF9b have been shown to bind to the same synaptic scaffold protein and to have a similar mode of action at inhibitory synapses (Woo et al. 2013). However, nothing is known about the function of IgSF9b or the interplay between IgSF9b and Nlgn2 in vivo, and particularly in the circuitry underlying anxiety or other psychiatrically related behaviors. Given their similar effects in cell cultures, I hypothesized that IgSF9b may modulate the function of Nlgn2 at inhibitory synapses in basal amygdala and exacerbate their anxiety related phenotype. To address this question, I combined behavioral testing, cFOS activation assay, neuronal tracing, quantitative immunohistochemistry and in vitro and in vivo electrophysiology in WT, Nlgn2 KO, IgSF9b KO and double KO mice. I showed that IgSF9b deletion rescues the anxiety-related phenotype of Nlgn2 KOs by modulating anxietyassociated activation and rescuing the deficit in inhibitory transmission of centromedial amygdala, the downstream target of basal amygdala. My findings identify a novel neural substrate of anxiety 


\section{RESULTS}

in centromedial amygdala and establish a novel modulator of Nlgn2 function in anxiety processing, thus expanding the current knowledge on circuit and molecular mechanisms of anxiety.

\section{Part 1- Characterization of the Anxiety Processing Circuit in Nlgn2 KOs, IgSF9b KOs and double KOs.}

\subsubsection{IgSF9b KO Rescues the Anxiety Phenotype of Nlgn2 KOs}

IgSF9b has been shown to localize to inhibitory synapses and reduce inhibitory transmission in cell cultures (Woo et al. 2013), suggesting that in vivo deletion of IgSF9b may exacerbate the inhibitory deficit and hence the anxiety phenotype in Nlgn2 KOs. To test this, I performed an open field test (OFT) on Nlgn2 KO, IgSF9b KO, double Nlgn2 IgSF9b KO and WT mice. Nlgn2 KO show a robust anxiety phenotype, making less entries and exploring the center significantly less than WT mice (Figure 3.2.1A-D), consistent with our previous findings (with an exception that albeit a strong trend, Nlgn2 KO did not spend significantly less time in the center of the open field than WTs (Figure 3.2.1D)). Surprisingly, IgSF9b KO show a significant increase in number of

A wT
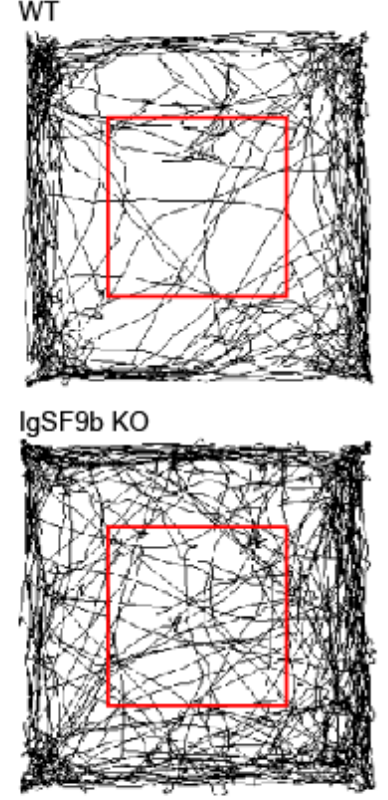

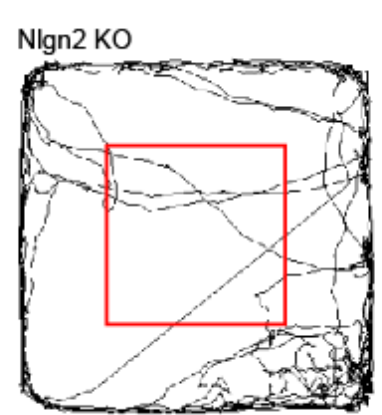

Double KO

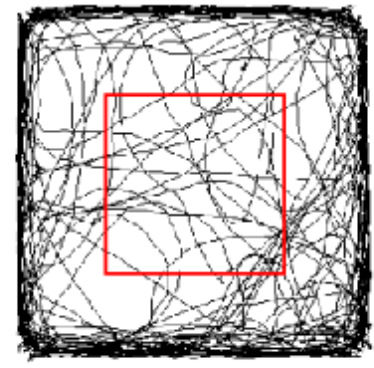

B

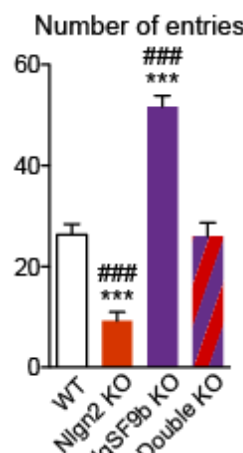

D Time in the center (s)

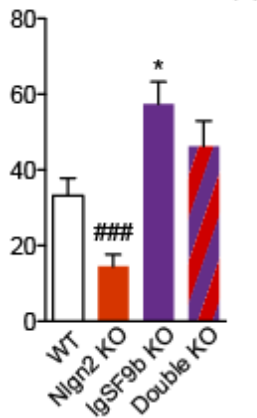

C Distance in center/total distance
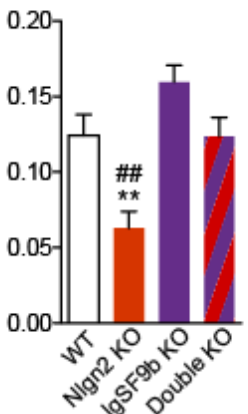

E

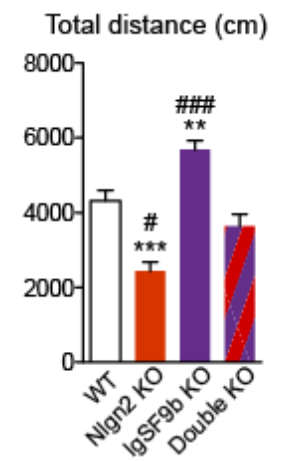




\section{RESULTS}

Figure 3.2.1| Anxiety phenotype and locomotor activity of Nlgn2 KO, IgSF9b KO and double KO mice in the open field chamber.

A. Representative tracks made by WT, Nlgn2 KO, IgSF9b KO and double KO during open field test. Red squares delineate the zone defined as a center of the open field.

B-D. Activity in the center zone of the open field chamber: total number of entries to the center (B), ratio between distance travelled in the center and total distance travelled in the open field $(\mathbf{C})$ and time spent in the center (D). Two-way ANOVA: (B) Main effect of Nlgn2 KO, $\mathrm{F}_{3,39}=94$, p < 0.0001; main effect of IgSF9b KO, $F_{3,39}=91.4, p<0.0001$. (C) Main effect of Nlgn2 KO, $F_{3,41}=15.92$, $p<0.0001$; main effect of IgSF9b KO, $\mathrm{F}_{3,39}=15.34, \mathrm{p}<0.0001$. (D) Main effect of $\mathrm{Nlgn} 2 \mathrm{KO}, \mathrm{F}_{3,42}=7.95, \mathrm{p}=0.0075$; main effect of IgSF9b KO, $\mathrm{F}_{3,42}=27.68, \mathrm{p}<0.0001$. No significant interaction between genotypes.

E. Total distance travelled in the open field chamber. Two-way ANOVA: Main effect of Nlgn2 KO, $\mathrm{F}_{3,41}$ $=51.86, \mathrm{p}<0.0001$; main effect of IgSF9b KO, $\mathrm{F}_{3,41}=22.28, \mathrm{p}<0.0001$. No significant interaction between genotypes. Post hoc one-way ANOVA with Bonferroni's correction: * $\mathrm{p}<0.05,{ }^{*} \mathrm{p}<0.01, * * * \mathrm{p}<0.001$ compared to WT; \# p $<0.05$, \#\# $\mathrm{p}<0.01$, \#\#\# $\mathrm{p}<0.001$ compared to double KO. $n=10-11$ for each genotype. All bars represent mean + SEM.

entries and time in the center of the open field (Figure 3.2.1A-B, D). Moreover, there is a complete rescue of the anxiety phenotype in double $\mathrm{KO}$ mice, which made similar number of entries, traveled the same normalized distance and spend same time in the center of the open field as WT mice (Figure 3.2.1A-D). While total distance traveled during OFT was significantly lower in Nlgn2 KO and significantly higher in IgSF9b KO compared to WT and double KOs (Figure 3.2.1E), this alteration of exploratory activity did not confound the anxiety phenotype, as evident in normalized activity in the center of the open (Figure 3.2.1C), which is significantly reduced in Nlgn2 KO and slightly but not significantly increased in IgSF9b KO. Taken together, these findings show that the prominent anxiety phenotype of Nlgn2 KOs may be rescued by deletion of IgSF9b, and that IgSF9b KO may suppress anxiety related processing in mice.

Based on empirical observation of Nlgn2 $\mathrm{KO}$ mice behavior in the OF, I hypothesized that the definition of the size of the center zone may mask the full extent of their anxiety- related phenotype. To establish whether Nlgn2 KO mice demonstrate anxiety- related behavior in more peripheral areas of the open field, I reanalyzed the behavioral data with respect to an intermediate zone (IZ), which I defined as a zone between the center and the area adjacent to the walls of the 


\section{RESULTS}

A

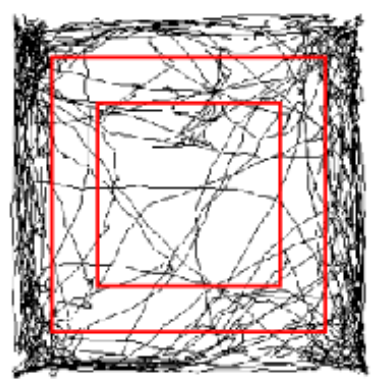

E

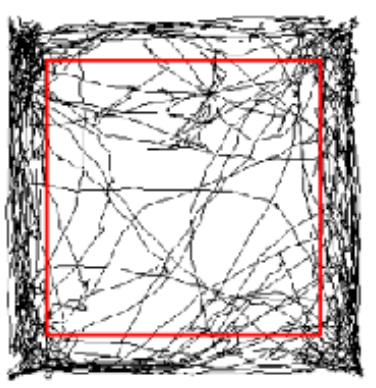

B

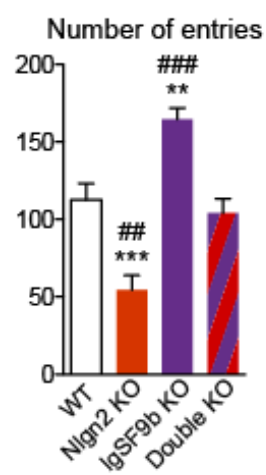

$\mathbf{F}$

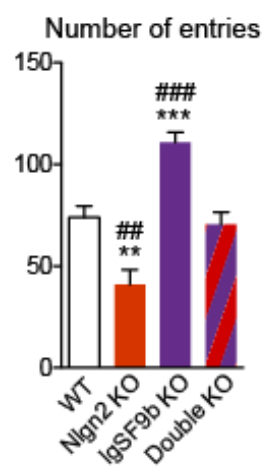

C

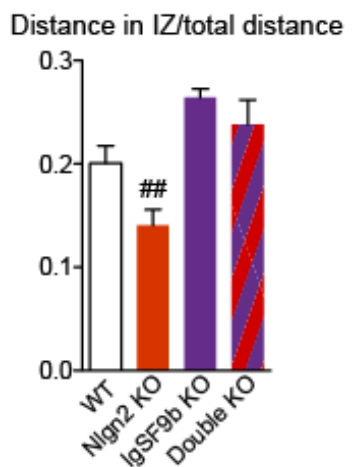

G

Distance in center/total distance

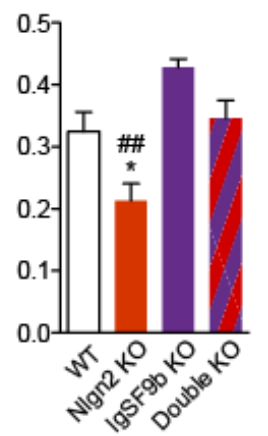

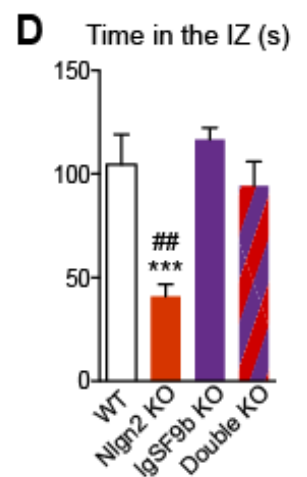

H Time in the center (s)

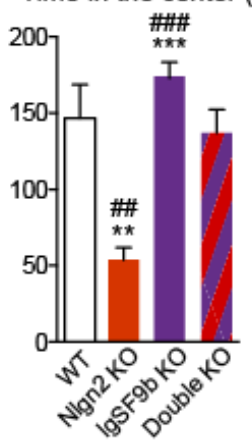

Figure 3.2.2 Anxiety phenotype of NIgn2 KO, IgSF9b KO and double KO mice in the intermediate zone and extended center of the open field.

A. Intermediate zone between the center of the OF and an area in immediate proximity to the walls (between two red squares).

B-D. Activity in the intermediate zone of the open field chamber: total number of entries to the center (B), ratio between distance travelled in the center and total distancet travelled in the open field $(\mathbf{C})$ and time spent in the center (D). Two-way ANOVA: (B) Main effect of Nlgn2 KO, $\mathrm{F}_{3,40}=41.19$, p < 0.0001; main effect of IgSF9b KO, $\mathrm{F}_{3,40}=30.10$, $\mathrm{p}<0.0001$. (C) Main effect of $\mathrm{Nlgn} 2 \mathrm{KO}, \mathrm{F}_{3,39}=6.34, \mathrm{p}=0.016$; main effect of IgSF9b KO, $\mathrm{F}_{3,39}=22.05, \mathrm{p}=0.0008$. (D) Main effect of Nlgn2 KO, $\mathrm{F}_{3,38}=17.288, \mathrm{p}=0.0002$; main effect of IgSF9b KO, $F_{3,38}=10.03, p=0.003$. No significant interaction between genotypes.

E. Redefined extended center zone that covers all the area of the open field chamber that is not in immediate proximity to the walls (red square).

F-H. Activity in the extended center zone of the open field chamber: total number of entries to the center $(\mathbf{F})$, ratio between distance travelled in the center and total distancet travelled in the open field $(\mathbf{G})$ and time spent in the center $(\mathbf{H})$. Two-way ANOVA: (B) Main effect of Nlgn2 KO, $\mathrm{F}_{3,41}=30.73$, p < 0.0001; main effect of IgSF9b KO, $\mathrm{F}_{3,41}=37.25$, p < 0.0001. (C) Main effect of Nlgn2 KO, $\mathrm{F}_{3,41}=20.04, \mathrm{p}<0.0001$; main effect of IgSF9b KO, $\mathrm{F}_{3,39}=13.34, \mathrm{p}=0.0008$. (D) Main effect of $\mathrm{Nlgn} 2 \mathrm{KO}, \mathrm{F}_{3,42}=13.13, \mathrm{p}=$ 0.0008; main effect of IgSF9b KO, $\mathrm{F}_{3,42}=18.18, \mathrm{p}=0.0001$. No significant interaction between genotypes. 


\section{RESULTS}

Post hoc one-way ANOVA with Bonferroni's correction: * $\mathrm{p}<0.05,{ }^{* *} \mathrm{p}<0.01,{ }^{* * *} \mathrm{p}<0.001$ compared to WT; \#\# p<0.01, \#\#\# p < 0.001 compared to double KO. $n=10-11$ for each genotype. All bars represent mean + SEM.

open field chamber (Figure 3.2.2A). Nlgn2 $\mathrm{KO}$ mice show strong thigmotaxis by making fewer entries and spending less time in the areas that are not adjacent to the walls of the open field. The extent of exploration of the IZ by double KO mice is again strikingly similar to that of WT mice (Figure 3.2.2B-D). As anxiety-related behavior of Nlgn2 $\mathrm{KO}$ and the rescue in double $\mathrm{KO}$ mice are evident both in IZ and the center of the open field, I merged IZ and the center zone into "extended center" (Figure 3.2.2E) and combined the behavioral data obtained from both zones. Anxiety- related phenotype of Nlgn2 $\mathrm{KO}$ mice is more robust in the extended center than in the initially used center of the open field (Figure 3.2.2F-H), suggesting that the previous definition of the center masked the full extent of the anxiety phenotype of Nlgn2 KOs. Therefore, I applied an extended center for the behavioral analysis of the consecutive experiments.

Finally, as all the previous experiments were conducted on male mice, I tested anxiety- related phenotypes of female Nlgn2 KO, IgSF9b KO and double KO mice. The phenotypes of females were similar to males (Figure 3.2.3A-C), demonstrating that sex does not modulate the effect of Nlgn2 KO and IgSF9b KO on anxiety.

\subsubsection{IgSF9b KO Rescues the Anxiety Associated Overactivation of Centromedial, but not Basal, Amygdala in Nlgn2 KO Mice}

To investigate the mechanism of anxiety rescue in double KOs, I first determined whether deletion of IgSF9b alters the anxiety- associated upregulation of basal amygdala in Nlgn2 KOs, and/or of adjacent regions that network with basal amygdala to mediate anxiety- related behavior, including lateral and central amygdala (Iwata et al. 1986; LeDoux et al. 1988; Tye et al. 2011) (Figure 3.2.4A). To this end, I exposed WTs, Nlgn2 KOs, IgSF9b KOs and double KOs to a novel environment (open field arena) and subsequently assessed cFOS activation of basal (BA), centromedial (CeM), lateral (LA) and centrolateral (CeL) amygdala. While the anxiety associated cFOS activation of LA and CeL is not altered in either Nlgn2 KO or IgSF9b KO (Figure 3.2.4E- 


\section{RESULTS}
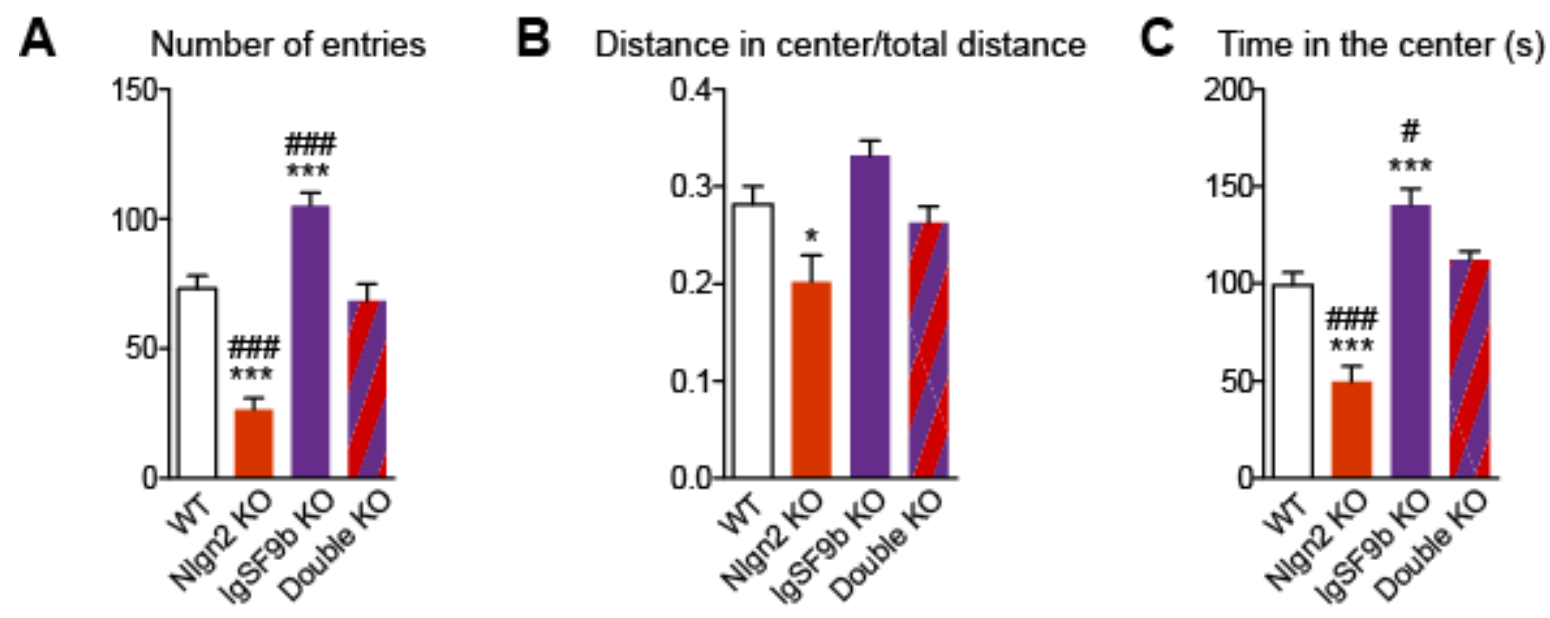

Figure 3.2.3 Anxiety phenotype of female NIgn2 KO, IgSF9b KO and double KO mice in the open field chamber.

A-C. Total number of entries to the center (A), ratio between distance travelled in the center and total distance travelled in the open field (B) and time spent in the center (C). Two-way ANOVA: (A) Main effect of Nlgn2 KO, $F_{3,49}=58.73, p<0.0001$; main effect of IgSF9b KO, $F_{3,49}=45.66, p<0.0001$. (B) Main effect of Nlgn2 KO, $\mathrm{F}_{3,48}=27.88, \mathrm{p}<0.0001$; main effect of IgSF9b KO, $\mathrm{F}_{3,48}=13.02, \mathrm{p}=0.0007$. (C) Main effect of Nlgn2 KO, $\mathrm{F}_{3,46}=30.47, \mathrm{p}<0.0001$; main effect of IgSF9b KO, $\mathrm{F}_{3,46}=54.27, \mathrm{p}<0.0001$. No significant interaction between genotypes.

Post hoc one-way ANOVA with Bonferroni's correction: * $\mathrm{p}<0.05,{ }^{* *} \mathrm{p}<0.01,{ }^{* * *} \mathrm{p}<0.001$ compared to WT; \# p < 0.05, \#\# p < 0.01, \#\#\# p < 0.001 compared to double KO. $n=10-11$ for each genotype. All bars represent mean + SEM.

F), all four genotypes show differential cFOS expression pattern in BA and CeM (Figure 3.2.4CD). Nlgn2 deletion results upregulation of cFOS expression in BA (Figure 3.2.4C) and particularly robustly in CeM (Figure 3.2.4D). Deletion of IgSF9b alone does not significantly alter the cFOS expression in any amygdala region, although there is a trend towards increase in BA (paired ttest, $\mathrm{p}=0.057$; Figure 3.2.4C-D). Strikingly, IgSF9b KO in Nlgn2 KOs robustly increases cFOS activation of BA (Figure 3.2.4C) and suppresses cFOS activation of CeM (Figure 3.2.4D). Taken together, these findings demonstrate that IgSF9b KO modulates the effect of Nlgn2 $\mathrm{KO}$ on anxiety associated activation of BA-CeM circuit. The following experiments aimed to elucidate the mechanism of this modulation. 


\section{RESULTS}

A
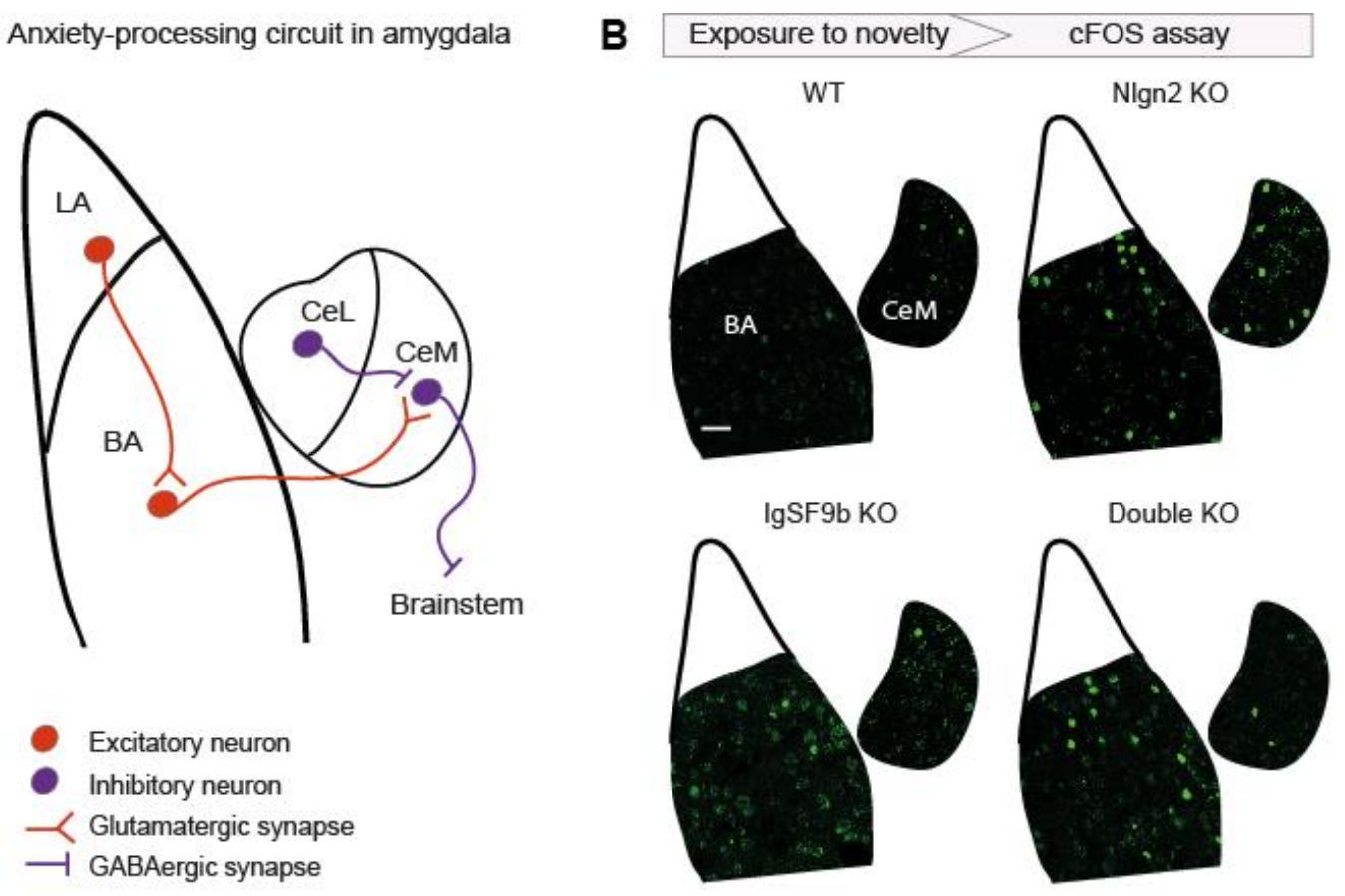

C
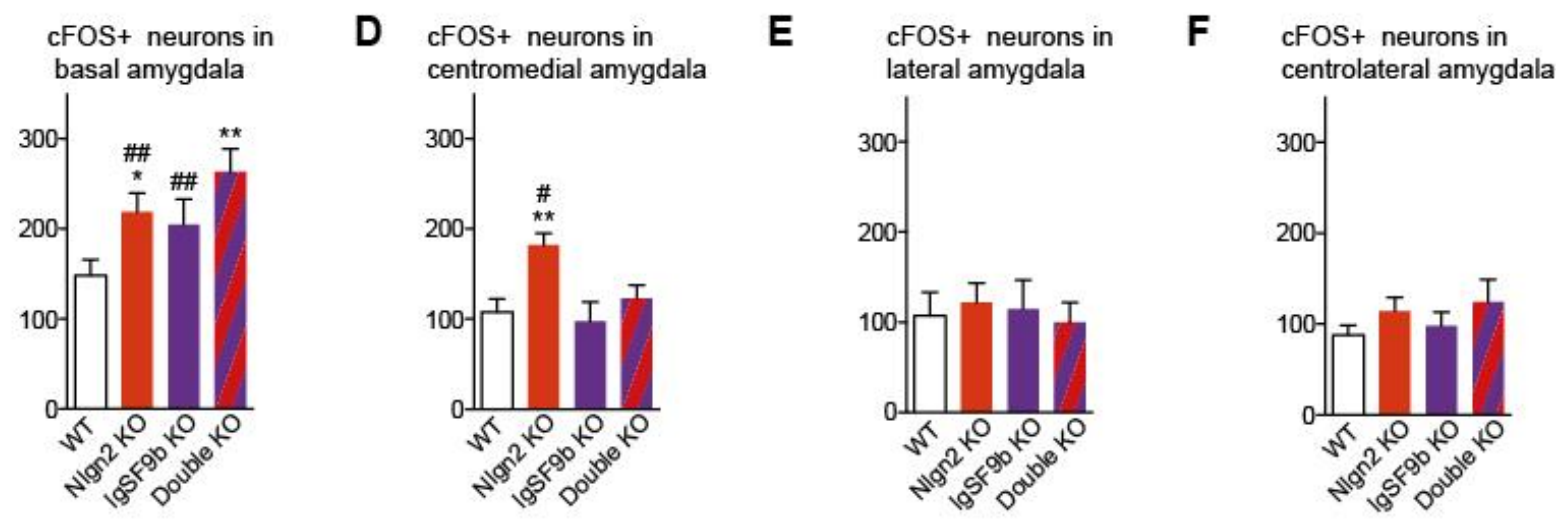

Figure 3.2.4 Anxiety-associated cFOS expression in distinct nuclei of amygdala in WT, NIgn2 KO,

\section{IgSF9b KO and double KO mice.}

A. Schematic representation of anxiety processing network in the amygdala.

B. Representative photomicrographs of cFOS expression in basal and centromedial amygdala. Scale bar, $50 \mu \mathrm{m}$. Here and below, cFOS expression was triggered by exposure to novel environment.

C-F. Total number of cFOS positive cells in basal (C), centromedial (D), lateral (E) and centrolateral (F) amygdala. Two-way ANOVA: (C) Main effect of Nlgn2 KO, $\mathrm{F}_{3,28}=7.58, \mathrm{p}=0.01$; main effect of IgSF9b $\mathrm{KO}, \mathrm{F}_{3,28}=4.58, \mathrm{p}=0.041$. (D) Main effect of Nlgn2 KO, $\mathrm{F}_{3,26}=9.47, \mathrm{p}=0.006$; main effect of IgSF9b $\mathrm{KO}, \mathrm{F}_{3,26}=0.044$. (E, F) No significant main effect of the genotype. No significant interaction between genotypes. Post hoc paired ttests: * $\mathrm{p}<0.05$, ** $\mathrm{p}<0.01$ compared to WT; \# $\mathrm{p}<0.05$, \#\# $\mathrm{p}<0.01$ compared to double KO. $n=8(\mathrm{C}), n=6(\mathrm{D}), n=5(\mathrm{E}, \mathrm{F})$ for each genotype. All bars represent mean + SEM. 


\section{RESULTS}

\subsubsection{IgSF9b KO Upregulates the Anxiety Associated Activation of Parvalbumin Neurons in Basal Amygdala}

IgSF9b KO may suppress the anxiety-associated activation of CeM in Nlgn2 KOs either by decreasing excitatory output from BA to $\mathrm{CeM}$ or by locally downregulating neural firing in CeM, or by both mechanisms. I first addressed the first possibility and investigated the effect of IgSF9b deletion on inhibitory circuitry in BA, specifically on anxiety-associated activation of interneurons that inhibit glutamatergic projection neurons during fear- related processing in BA, such as Parvalbumin (PV) and Somatostatin (SOM) (Wolff et al. 2014; summarized in Figure 5A). To this end, I performed double labeling of cFOS and PV or SOM in Nlgn2 KOs, IgSF9b KOs, double KOs and WTs after their exposure to an anxiogenic environment. IgSF9b KO enhances the activation of PV neurons in BA both in single IgSF9b KOs and, more robustly, in double KOs (Figure 3.2.5B-C), and does not alter the activation of SOM neurons (Figure 3.2.5F). The total number of PV neurons was increased in Nlgn2 KOs, as shown previously, but did not significantly differ between WT, IgSF9b KOs and double KOs (Figure 3.2.5D). This finding suggests that deletion of IgSF9b enhances the activation of PV network that may decrease the excitatory output of glutamatergic neurons during anxiety- related processing.

\subsubsection{IgSF9b KO Does Not Downregulate the Excitatory Output from Basal Amygdala to Centromedial Amygdala in Nlgn2 KOs}

Next I determined whether Nlgn2 KO or IgSF9b KO modulate anxiety-associated activation of glutamatergic neurons in BA that project to $\mathrm{CeM}$. To label CeM projectors, which do not express distinctive cellular markers and are intermingled with other glutamatergic neurons in BA (Namburi et al. 2015), I established a retrograde tracing technique that allows the delivery of fluorescent beads injected to CeM to the cell bodies of neurons in BA that project to CeM (Figure 3.2.6A-C). A week after the injection of beads into CeM, Nlgn2 KOs, IgSF9b KOs, double KOs and WTs were exposed to novel environment followed by cFOS labeling of BA. While more data points must be added to reach statistical significance, there is a robust trend towards increase in number of beads- labeled neurons that express cFOS in all three genotypes, that is particularly robust in 
A Neuronal components of inhibitory network in basal amygdala

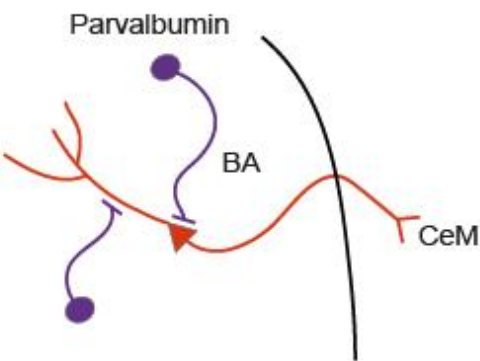

Somatostatin

A Pyramidal- like neuron

- Inhibitory interneuron

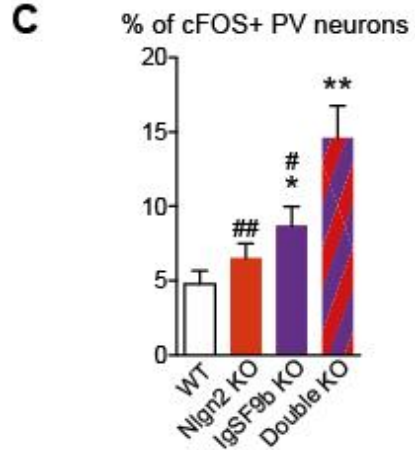

$\mathbf{F}$

$\%$ of cFOS+ Som neurons

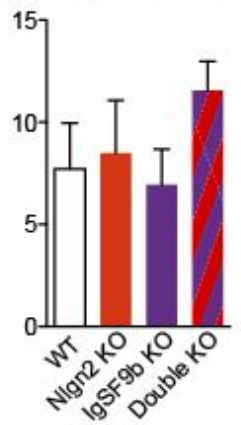

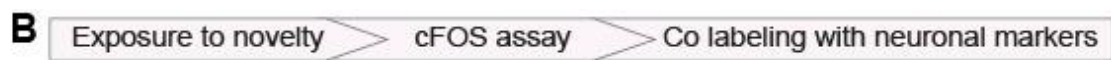
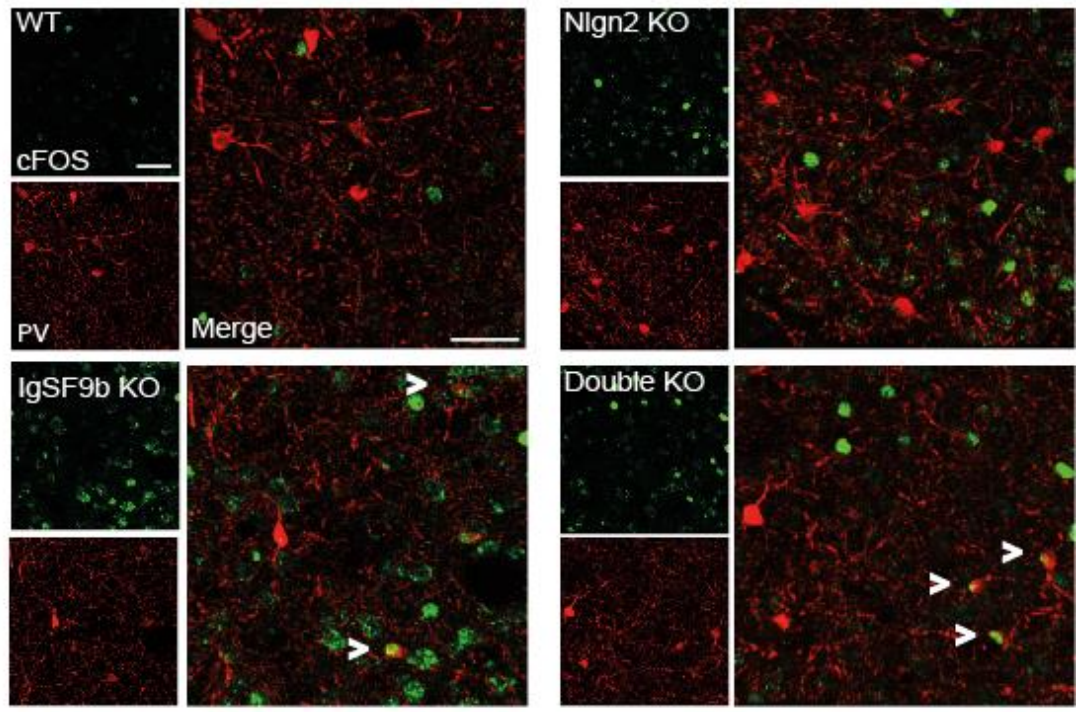

D Total number of PV neurons
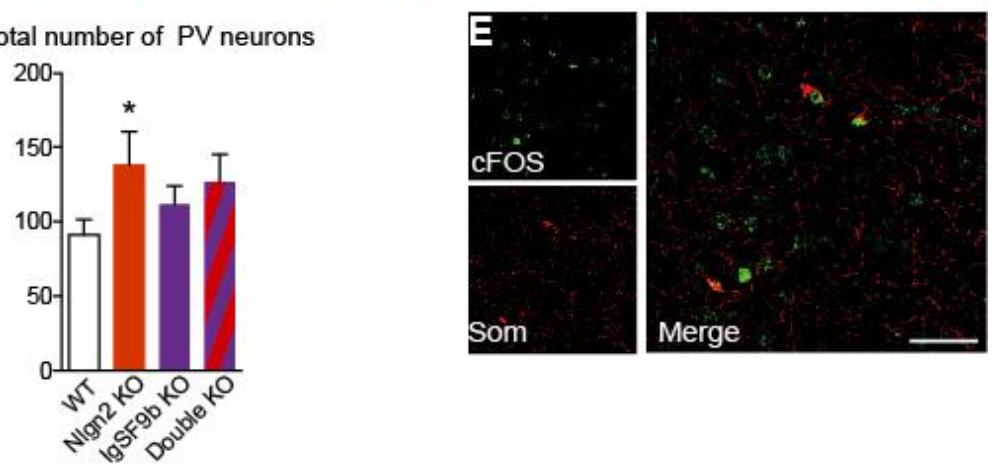

Figure 3.2.5| Effect of IgSF9b deletion on anxiety- associated activation of inhibitory network in basal amygdala in WT and Nlgn2 KO mice.

A. Schematic representation of neuronal components of local inhibitory network in basal amygdala.

B, E. Photomicrographs of cFOS and Parvalbumin colocalization in basal amygdala of WT, Nlgn2 KO, IgSF9b $\mathrm{KO}$ and double $\mathrm{KO}$ (B) and representative photomicrograph of cFOS and Somatostatin colocalization in basal amygdala of WT $(\mathbf{E})$. Arrows indicate cFOS positive Parvalbumin neurons. Here and below cFOS expression was triggered by exposure to novel environment. Scale bar, $50 \mu \mathrm{m}$. 


\section{RESULTS}

C-D. Total number of Parvalbumin neurons (C) and percentage of Parvalbumin neurons that express cFOS out of total number of Parvalbumin neurons (D) in basal amygdala. Two-way ANOVA: (C) Main effect of Nlgn2 KO, $\mathrm{F}_{3,28}=3.5, \mathrm{p}=0.07$ (a trend), no significant main effect of IgSF9b KO. (D) Main effect of $\mathrm{Nlgn} 2 \mathrm{KO}, \mathrm{F}_{3,28}=7.17, \mathrm{p}=0.012$; main effect of IgSF9b KO, $\mathrm{F}_{3,28}=17.66, \mathrm{p}=0.0002$. No significant interaction between genotypes. Post hoc paired ttests: * $\mathrm{p}<0.05$, ** $\mathrm{p}<0.01$, compared to WT; \# $\mathrm{p}<0.05$, \#\# $\mathrm{p}<0.01$, compared to double KO. $n=8$ for each genotype.

F. Percentage of Somatostatin neurons that express cFOS out of total number of Somatostatin neurons in basal amygdala. No significant main effect of the genotype. No significant interaction between genotypes. $n=7$ for each genotype. All bars represent mean + SEM.

Nlgn2 KO and double KO (Figure 3.2.6D-E). This demonstrates that Nlgn2 KO upregulates anxiety associated activation of BA excitatory output to CeM, which is consistent with increased cFOS expression in CeM of Nlgn2 KOs. Surprisingly, although IgSF9b deletion upregulates inhibitory PV network in BA, it does not suppress the activation of CeM projectors in Nlgn2 KOs, indicating that rescue of anxiety associated CeM activation in double KOs is mediated downstream of BA by a local mechanism in CeM.

\subsubsection{Centromedial Amygdala Mediates Anxiety Related Processing In Vivo}

Higher cFOS activation of CeM in "anxious" Nlgn2 KO mice compared to "non-anxious" double $\mathrm{KO}$ mice indicates that neural activity in CeM may be associated with anxiety- related behavior, and that IgSF9b KO may modulate this activity to rescue the anxiety- related phenotype of Nlgn2 KO mice. Therefore, I hypothesized that the extent of activation of CeM during exploration of the OF correlates with the extent of avoidance of the center zone, and that neural activity in CeM differentially encodes "safe" (periphery) and "danger" (center) zones across genotypes. As cFOS activation assay does not provide with temporal resolution necessary to measure neural activity in different compartments of the open field, I sought to assess neural activity of CeM while the mice explore different compartments of OF by recording local field potential (LFP) in CeM in vivo. The recording and data analysis was performed by Dr. Hugo Cruces- Solis at the Department of Molecular Neurobiology, Max Planck Institute for Experimental Medicine. 
A
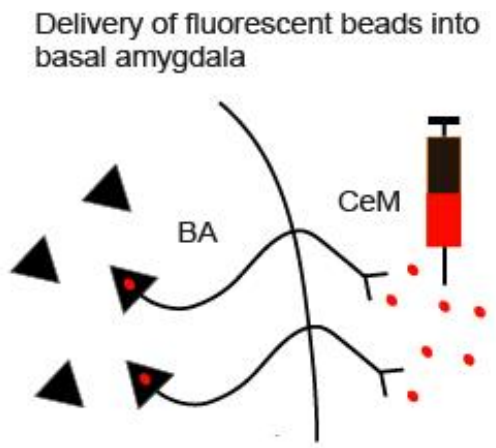

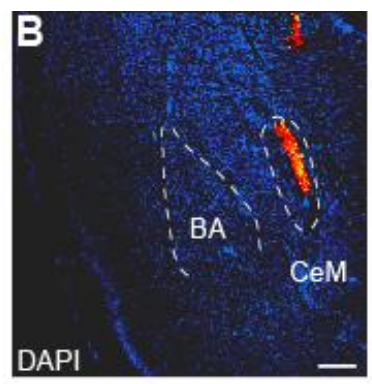

DAPI
D
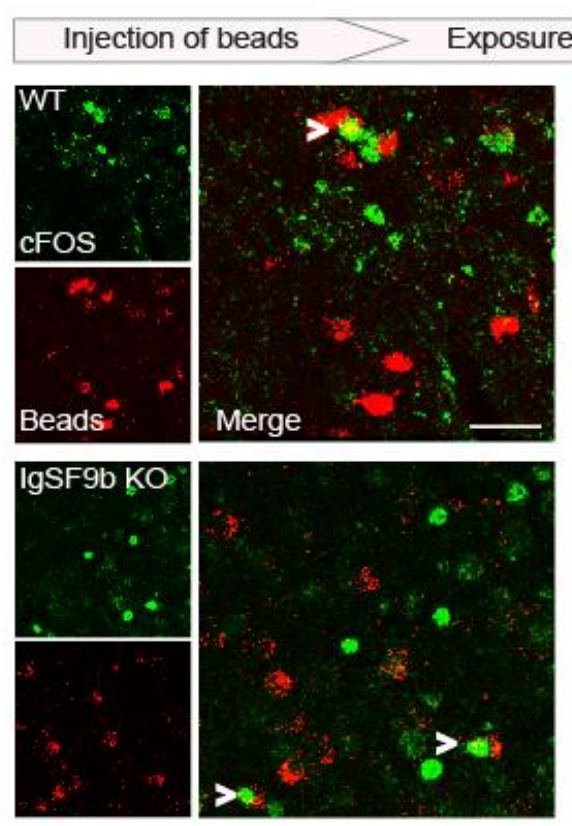
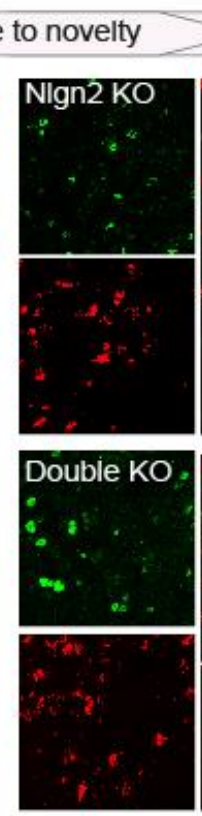
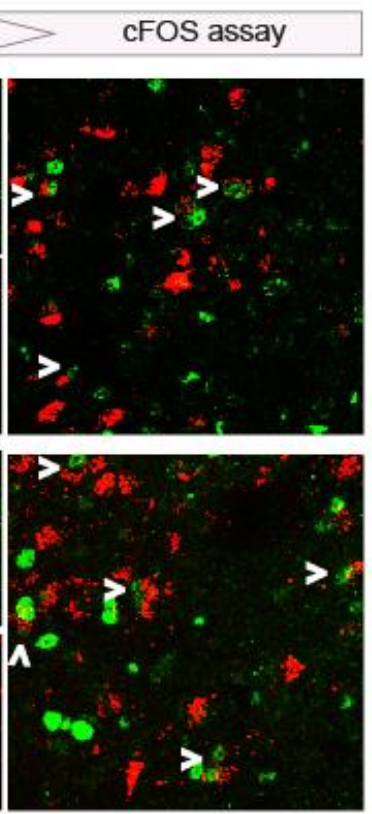

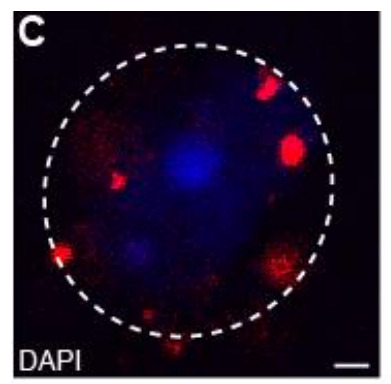

E $\%$ of cFOS+ neurons from WT level

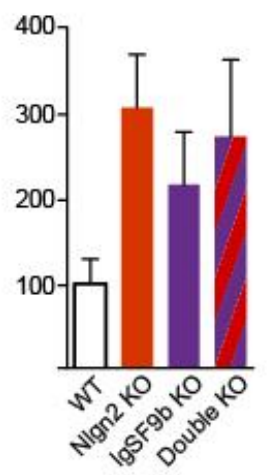

Figure 3.2.6 Effect of NIgn2 and IgSF9b KO on anxiety- associated activation of glutamatergic neurons in basal amygdala that project to centromedial amygdala.

A. Schematic representation of delivery of retro beads into basal amygdala following injection of retro beads to centromedial nucleus.

B-C. Photomicrograph showing the injection site of retro beads in the centromedial amygdala $(\mathbf{B})$ and high magnification photomicrograph showing the expression of red beads in the cell body (delineated with white circle) of a neuron in basal amygdala following migration of the beads from centromedial amygdala (C). Scale bar, $200 \mu \mathrm{m}, 2 \mu \mathrm{m}$.

D. Photomicrographs of cFOS and retro beads colocalization in basal amygdala of WT, Nlgn2 KO, IgSF9b KO and double KO mice. Arrows indicate cFOS positive neuron bodies filled with retro beads. Scale bar, $50 \mu \mathrm{m}$.

E. Percentage of beads- containing neurons that express cFOS out of total number of beads- containing neurons in basal amygdala, relative to the baseline. The baseline was set as average percentage in WT at 100\%. $n=3-4$. All bars represent mean + SEM. 


\section{RESULTS}
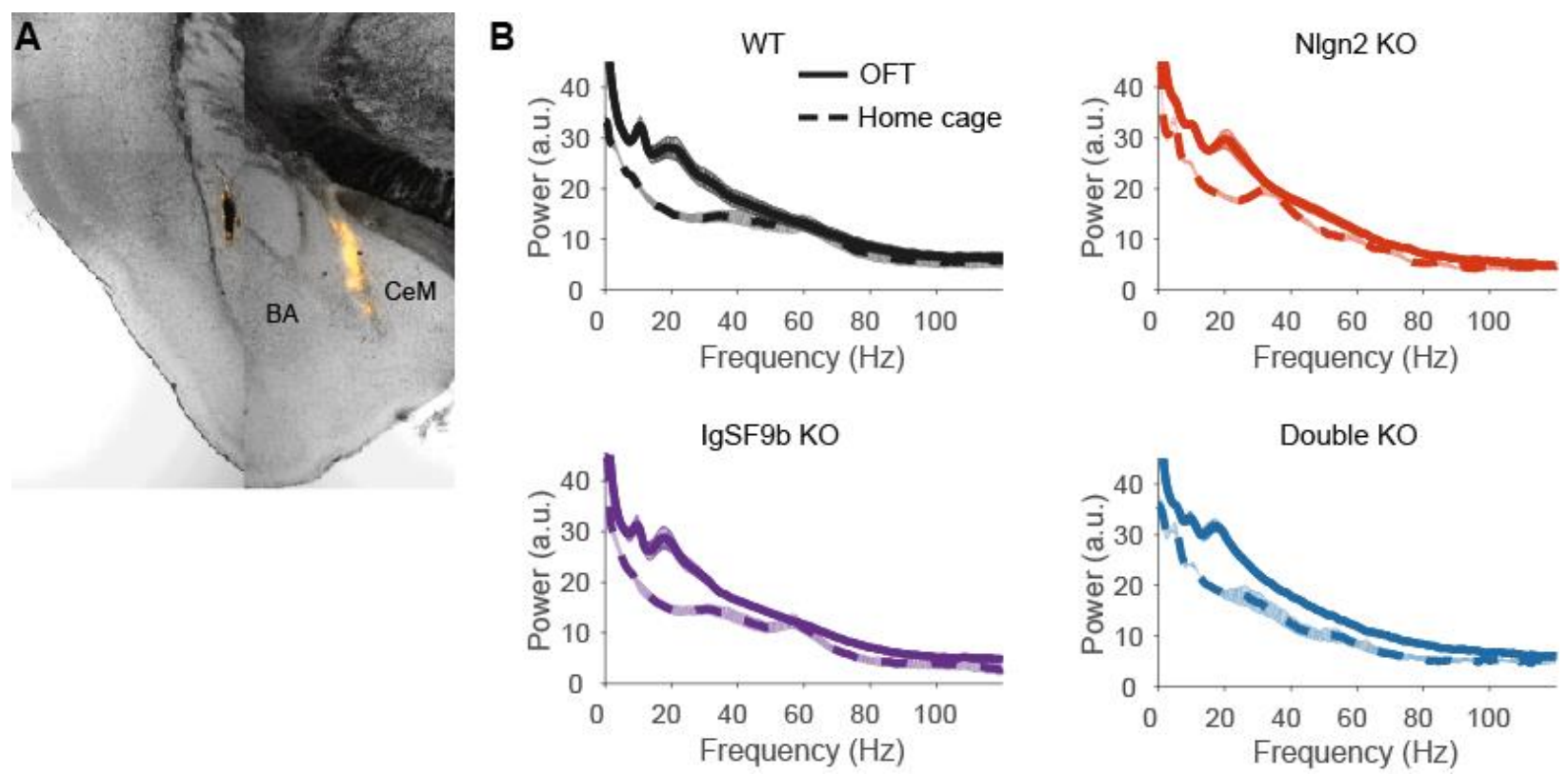

Experiment performed by Dr. Hugo Cruces Solis

Figure 3.2.7| Effect of exposure to the open field on CeM neural activity in WT, NIgn2 KO, IgSF9b KO and double KO.

A. Photomicrograph showing the recording site in CeM (yellow mark).

B. Average power spectra of CeM LFP obtained in the home cage (dashed line) and during 10 minutes of OF exploration (solid line).

One week after the implantation of the electrodes in the CeM (Figure 3.2.7A), LFPs were recorded in the home cage and during 15 minutes of OFT in Nlgn2 KO, IgSF9b KO, double KO and WT mice. First, we tested whether exposure to novel environment increases neural activation in CeM in all genotypes by comparing the LFPs in home cage vs LFPs in OFT. Power spectra analysis of the LFPs revealed that exposure to the OF chamber robustly increases the power across the frequency range analyzed (Figure 3.2.7B) in WT, Nlgn2 KO, IgSF9b KO and double KOs. This OF- induced increase demonstrates that $\mathrm{CeM}$ is activated by anxiogenic conditions.

\subsubsection{IgSF9b KO Rescues the Enhanced Anxiety Associated Activation of CeM in Nlgn2 KOs}

Next, we tested whether deletion of Nlgn2 and/or IgSF9b differentially modulates neural activity in CeM during exploration of the OFT. To this end, we analyzed separately the recording obtained 


\section{RESULTS}

A

Neural activity in the center of the OF

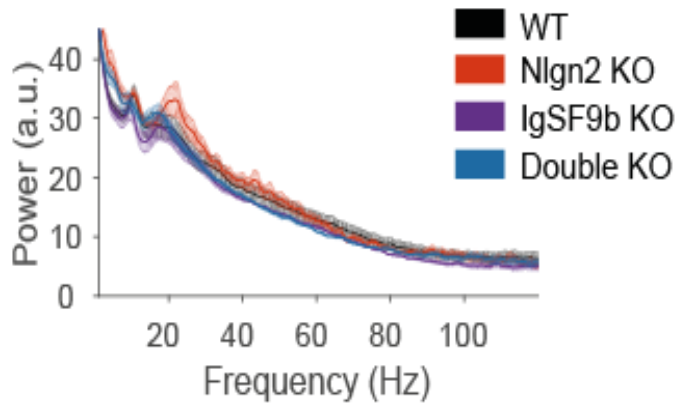

B Neural activity in the periphery of the OF

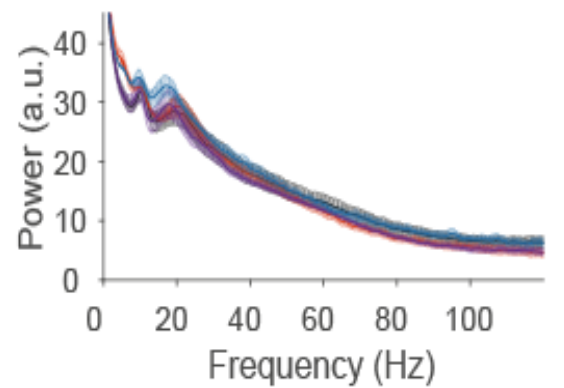

Figure 3.2.8| CeM activity during exploration of periphery and center of the $\mathrm{OF}$.

A-B. Average power spectra of CeM LFP in the center (A) and in the periphery of the OF (B). $n=6$ for WT and IgSF9b KO, $n=4$ for Nlgn2 KO and $n=3$ for double KO.

while mice were exploring the periphery vs the center of the OF. Nlgn2 KO demonstrate a slight increase in LFP of CeM during exploration of the center of the OF compared to IgSF9b KOs, double KOs and WTs (Figure 3.2.8A), while the LFP during exploration of the periphery is similar in all genotypes (Figure 3.2.8B). To further investigate this center- specific increase in LFP power of Nlgn2 KO, we analyzed the transitions as the mice approached and explored the center. This analysis is typically used to assess dynamic change in neural activity during exploration of the open field (Likhtik et al. 2013; Stujenske et al. 2014). For each transition, we compared the LFP power relative to the baseline (average power of LFP in the periphery) for each time point and plotted the magnitude of change in average LFP power across all trials. This analysis revealed that Nlgn2 deletion robustly increases the power of LFP during exploration of the center of OF across all frequency ranges (Figure 3.2.9A).

Strikingly, this increase is rescued in the double KO at high gamma frequency range $(70-120 \mathrm{~Hz})$, as evident in direct comparison of change in high gamma LFP in all genotypes (Figure 3.2.9B). To quantify the magnitude of center- induced change of high gamma band, we averaged the high gamma power across each trial. We observed >10- fold center- induced increase in gamma frequency power in Nlgn2 $\mathrm{KO}$ mice, as well as a small increase in double $\mathrm{KO}$ that corresponded 


\section{RESULTS}

A

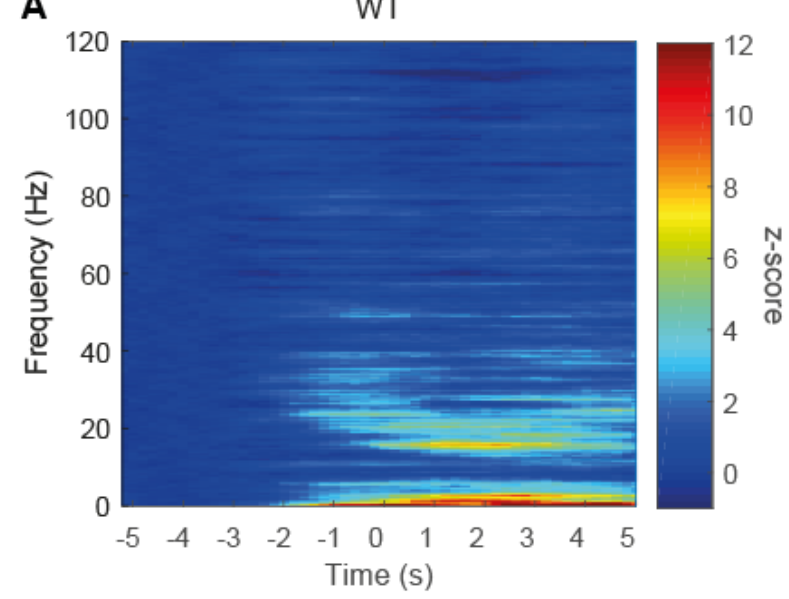

IgSF9b KO

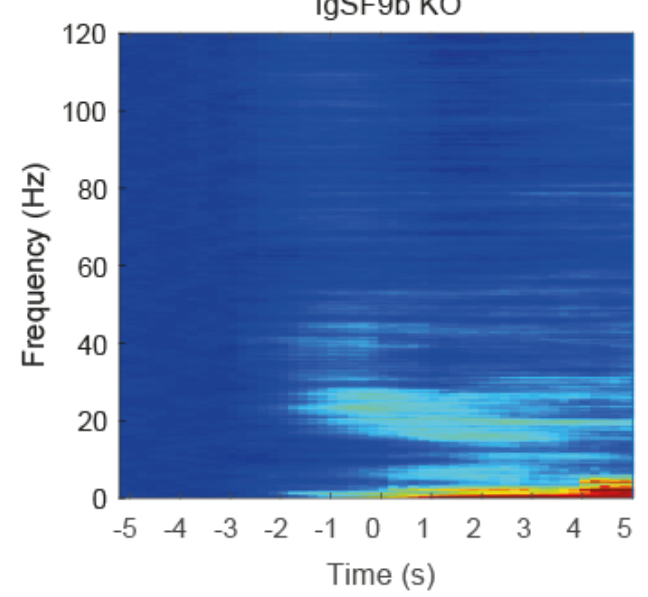

B

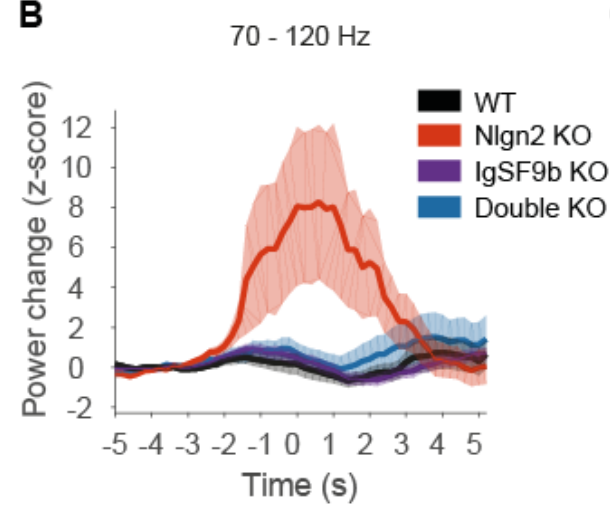

C Average Z- Score

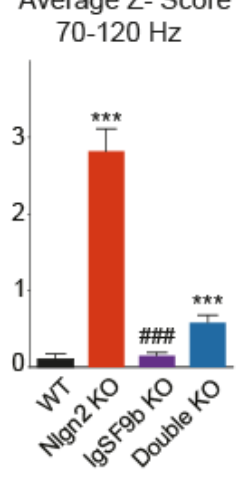

Nlgn2 KO

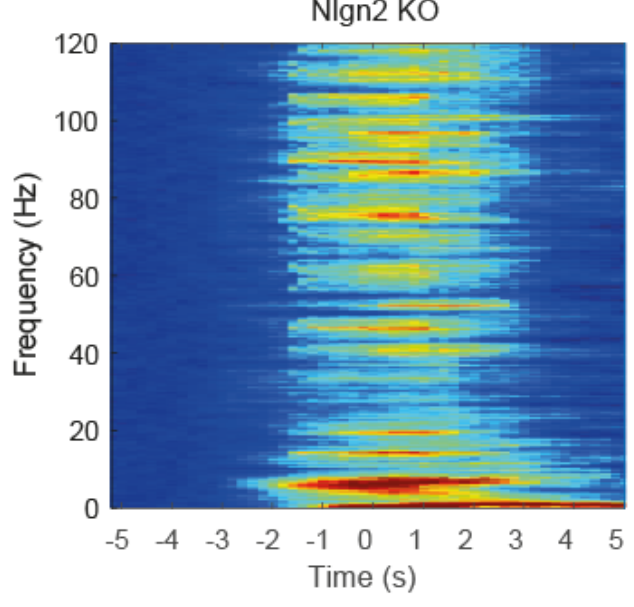

Double KO

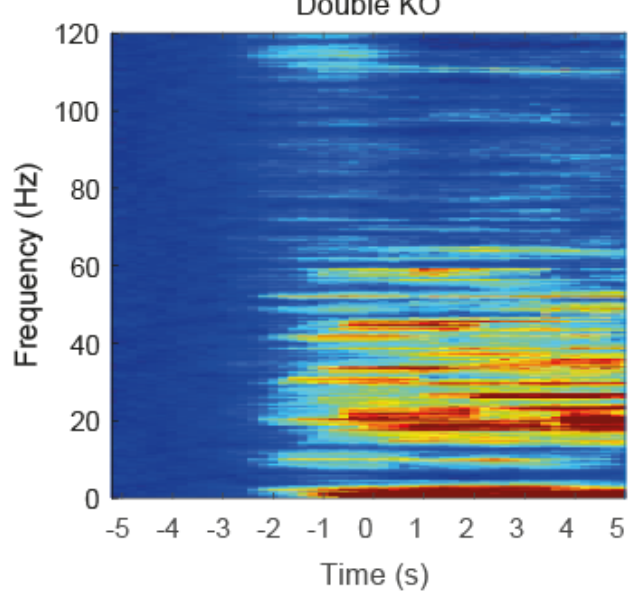

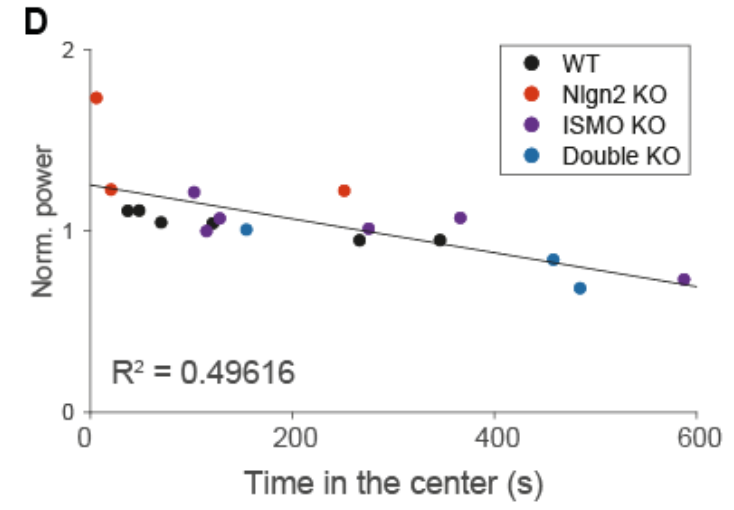

Experiment performed by Dr. Hugo Cruces Solis 


\section{RESULTS}

Figure 3.2.9| Effect of Nlgn2 KO on CeM activity during center-periphery transitions and correlation of CeM activity to anxiety- like behavior.

A. Normalized power change during transitions from periphery to the center, averaged across transitions and plotted as a function of time (time point in which the mouse is closest to the center is designated as zero, $5 \mathrm{~s}$ of data on both sides of zero are shown). Baseline defined as average LFP power obtained during first three seconds of transition to the center.

B-C. Normalized 70-120 Hz power change during 10 seconds of center-periphery transition (B) and its mean (taken from 52 data points for each mouse) (C). Two-way ANOVA: (C) Main effect of Nlgn2 KO, $\mathrm{F}_{3,207}=50.63, \mathrm{p}<0.0001$; Main effect of IgSF9b KO, $\mathrm{F}_{3,207}=25.29, \mathrm{p}<0.0001$; Interaction between genotypes,

$\mathrm{F}_{3,207}=27.15, \mathrm{p}<0.0001$. Post hoc paired ttests: $* * * \mathrm{p}<0.01$, compared to WT, \#\#\# $\mathrm{p}<0.01$, compared to double KO. $n=6$ for WT and IgSF9b KO, $n=4$ for Nlgn2 KO and $n=3$ for double KO.

D. Linear correlation coefficient of the normalized CeM high gamma power during all center transitions with total time of each mouse in the center of the OF. $\mathrm{p}=0.0007, n=18$.

to $15 \%$ of the increase in Nlgn2 $\mathrm{KO}$ (Figure 3.2.9C). This demonstrates that Nlgn2 deletion increases gamma band activity in CeM during anxiogenic exploration of the center and that IgSF9b deletion in Nlgn2 KOs almost completely rescues this increase.

Finally, we tested whether the increase in normalized power in high gamma range correlates with anxiety- like phenotype in the OFT. To this end, we plotted normalized power as a function of time spent in the center of the OF by all mice in the study. The less time a mouse spends in the center, the higher is the change in high gamma band in the center, with a significant negative correlation (Figure 3.2.9D), thus linking high gamma band activity of CeM to behavioral manifestation of anxiety.

Taken together, our findings confirm the rescue of anxiety related activation of CeM in double KO and demonstrate that IgSF9b deletion normalizes the anxiety- related behavior of Nlgn2 KOs in OFT by suppressing the increase in gamma band activity of CeM. As increase in power of LFP indicates increase in synaptic input (Buzsáki et al. 2012), increase in LFP power in Nlgn2 KOs is consistent with their increased anxiety-associated excitatory input to the CeM and enhanced cFOS expression in CeM. 


\section{RESULTS}

\subsubsection{Summary and Conclusions - Part 1}

The goal of the present study was to elucidate the circuit mechanism by which deletion of novel synaptic adhesion protein IgSF9b rescues the anxiety related phenotype of Nlgn2 KOs. To this end, I show that IgSF9b KO modulates the enhancing effect of Nlgn2 KO on anxiety-associated activation of basal and centromedial amygdala. Specifically, IgSF9b KO elevates cFOS expression in basal amygdala and reduces cFOS expression in centromedial amygdala in Nlgn2 KOs. Dissecting the cFOS expression pattern in basal amygdala to neural components revealed that although IgSF9b KO upregulates anxiety-associated activation of the inhibitory Parvalbumin network, it does not suppress the excitatory output of basal amygdala to centromedial amygdala in Nlgn2 KOs, indicating that the rescue of anxiety related phenotype is predominantly mediated by local mechanism in centromedial amygdala. To further investigate this rescue in vivo, we performed recordings of local field potentials of centromedial amygdala in mice under anxiogenic conditions. Analysis of these recordings showed that IgSF9b KO decreases the activity of centromedial amygdala in gamma frequency range $(70-120 \mathrm{~Hz})$ in Nlgn2 KOs, and that the magnitude of gamma band activity in centromedial amygdala directly correlates with the extent of anxiety related phenotype.

Taken together, these findings demonstrate that IgSF9b KO rescues the anxiety related phenotype of Nlgn2 KO by modulating anxiety associated neural activity in centromedial amygdala in vivo. Following experiments aimed to characterize the cellular and molecular mechanism of this modulation. 


\section{RESULTS}

\section{Part 2- Molecular Characterization of Nlgn2 KO and IgSF9b KO in Basal and Centromedial amygdala.}

\subsubsection{IgSF9b is Widely Expressed in the Brain}

To begin to address the cellular mechanisms underlying the rescue of anxiety-associated activation in CeM in double KOs, I first examined the expression pattern of IgSF9b in the brain of adult mouse. IgSF9b is expressed in many brain regions, including cortex, striatum, amygdala and cerebellum and, to a lesser extent in hippocampus (Figure 3.2.10), similarly to its expression pattern in rat brain (Woo et al. 2013).

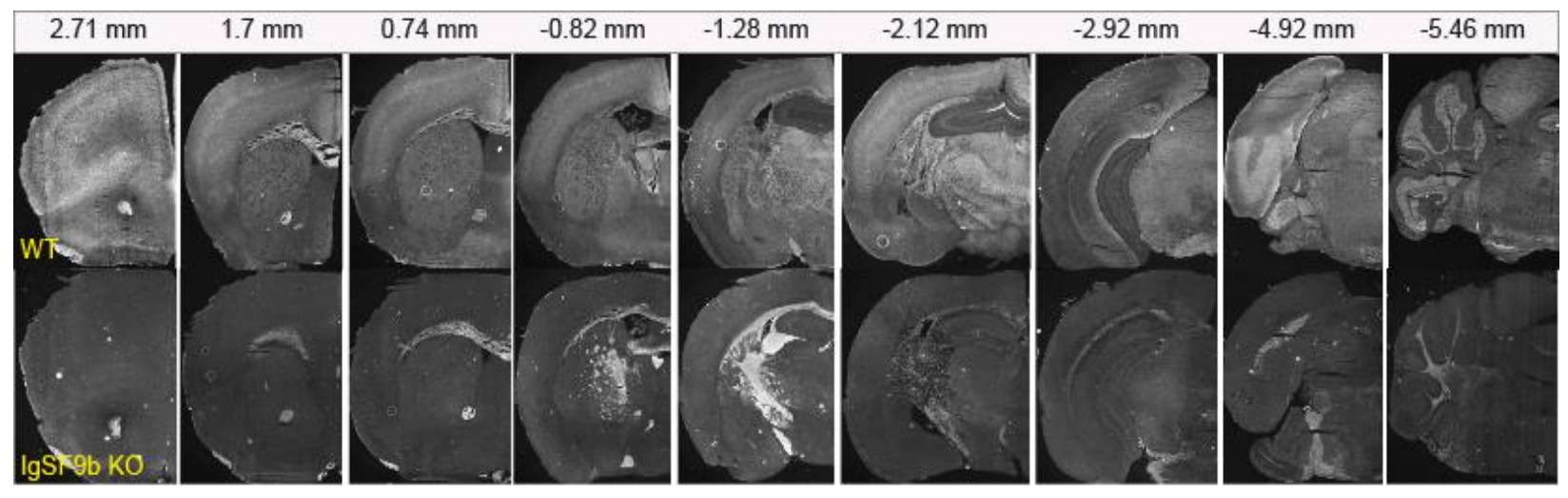

Figure 3.2.10| IgSF9b expression in the brain.

Low magnification photomicrographs of coronal sections show IgSF9b expression in WT mouse (upper panel) and non- specific binding of IgSF9b antibody in IgSF9b KO (lower panel). Anterio- posterior coordinates from Bregma are indicated in the uppermost panel.

\subsubsection{IgSF9b Does Not Colocalize with Synaptic Markers In Vivo}

Next, I characterized the synaptic expression of IgSF9b in BA. For this purpose, I performed double labeling of IgSF9b with synaptic markers gephyrin, Nlgn2, PSD- 95 and VGAT. IgSF9b 


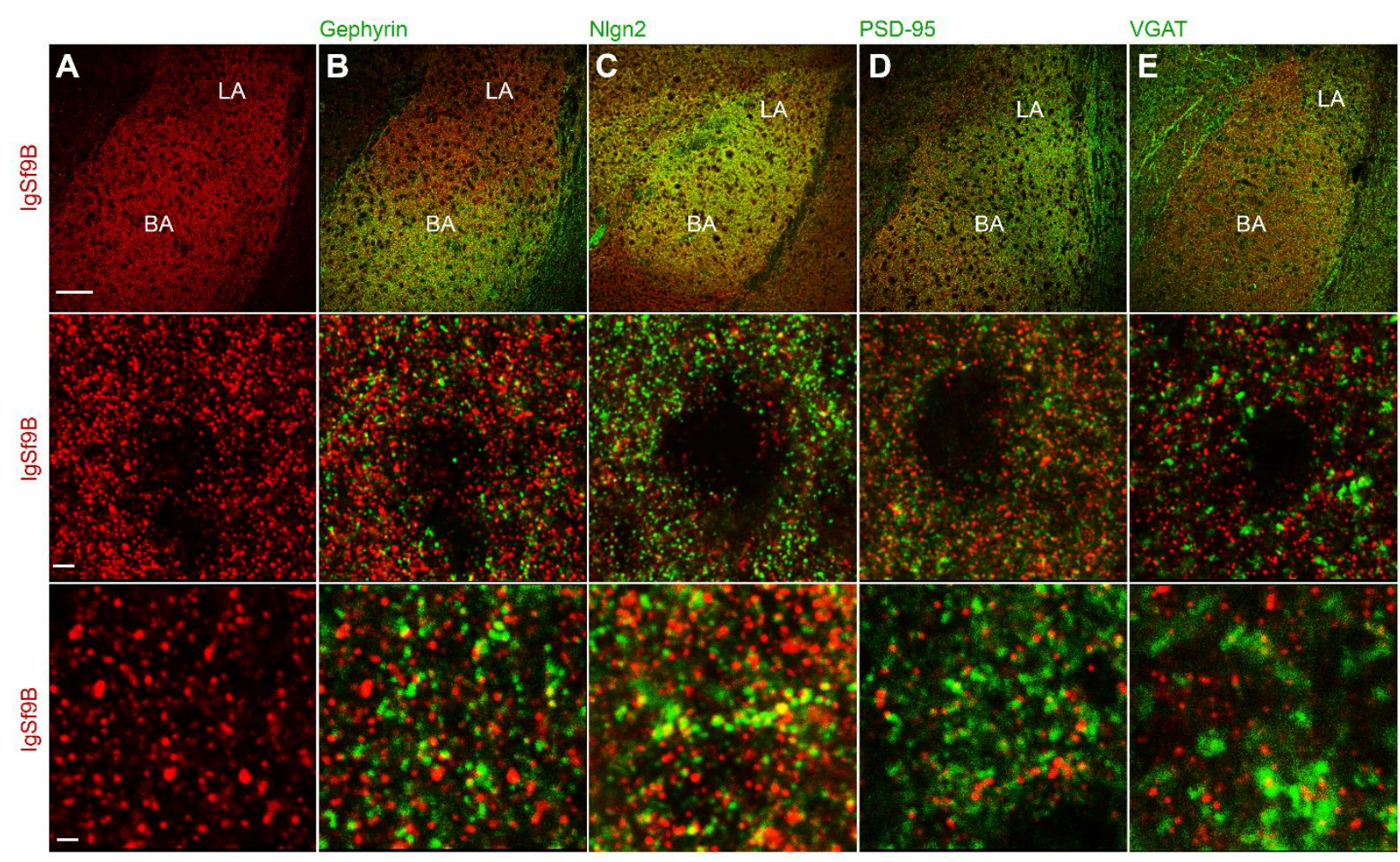

Figure 3.2.11| IgSF9b expression and colocalization with synaptic markers in basal amygdala.

A. Photomicrographs showing IgSF9b expression in basal amygdala (upper panel), IgSF9b localization at cell membrane (middle panel) and IgSF9b puncta (lower panel).

B-E. Photomicrographs showing low degree of colocalization with post synaptic markers gephyrin (B), Nlgn2 (C), PSD- 95(D) and presynaptic marker VGAT (E). Scale bars, $100 \mu \mathrm{m}$ (upper), $2 \mu \mathrm{m}$ (middle) and $1 \mu \mathrm{m}$ (lower).

is highly expressed in basal amygdala; its punctate appearance and delineation of the cell body suggests that IgSF9b is a synaptic protein (Figure 3.2.11A). IgSF9b is apposed but does not fully overlap with any of the synaptic markers analyzed, although partial overlapping with gephyrin and Nlgn2 may be observed (Figure 3.2.11B-E). This result resembles the expression pattern of IgSF9b in cultured neurons, where clusters of IgSF9b were shown to be closely apposed but not to fully overlap with gephyrin clusters (Woo et al. 2013), supporting the notion that IgSF9b is localized in the periphery of inhibitory synapses and not at the core of inhibitory postsynaptic sites that contain gephyrin and GABA receptors. 


\section{RESULTS}
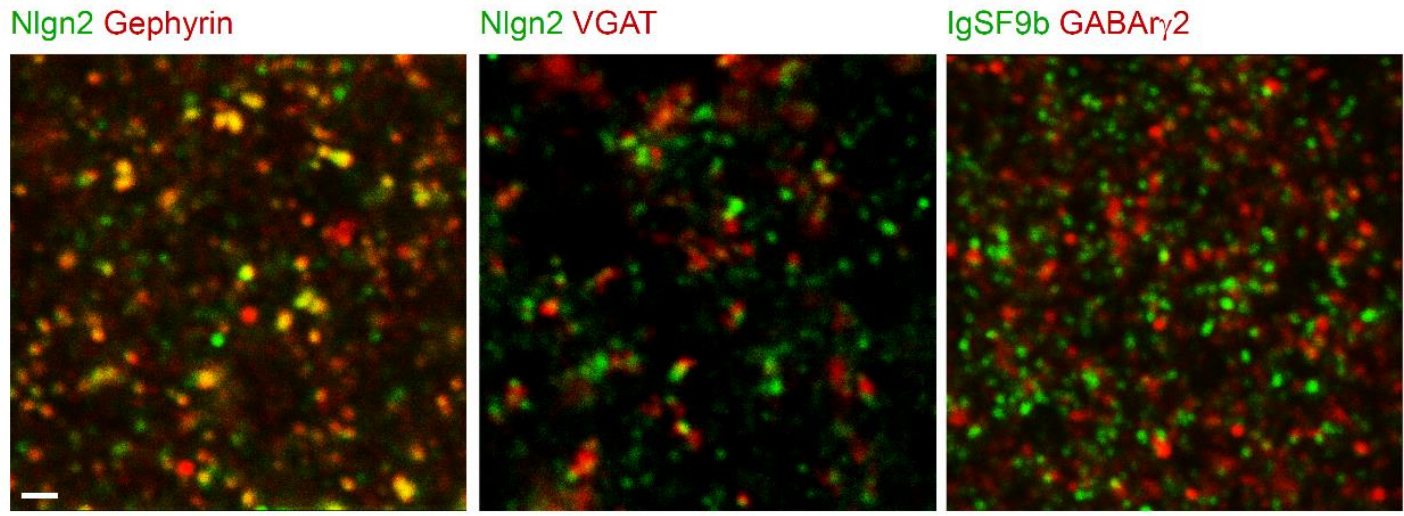

Figure 3.2.12| Colocalization of IgSF9b with $\gamma 2$ subunit of GABA receptor in centromedial amygdala compared to colocalization of Nlgn2 with inhibitory markers.

Photomicrograph of Nlgn2 and gephyrin puncta (left) shows a high degree of colocalization of two directly interacting proteins. Nlgn2 and VGAT (middle) puncta do not colocalize but opposed to each other, similarly to IgSF9b and GABAr 2 puncta in CeM (right). Scale bar, $1 \mu \mathrm{m}$.

\subsubsection{IgSF9b is Apposed to Gamma 2 Subunit of GABA Receptor in Centromedial Amygdala}

IgSF9b was shown to be tightly associated with Gamma 2 subunit of GABA receptor (GABAr 2 subunit) at the postsynaptic sites (Woo et al. 2013). Therefore, I investigated the degree of the colocalization between IgSF9b and GABAry2 subunit in CeM. I compared double-labelled IgSF9b and GABAry2 puncta to highly colocalized Nlgn2 and gephyrin puncta and to closely apposed Nlgn2 and VGAT puncta. IgSF9b-GABAry2 and Nlgn2-VGAT have similar colocalization patterns (Figure 3.2.12), suggesting that unlike gephyrin and Nlgn2, IgSF9b does not directly interact with GABA receptors and is localized in close apposition to inhibitory synapses in CeM. 


\section{RESULTS}

A
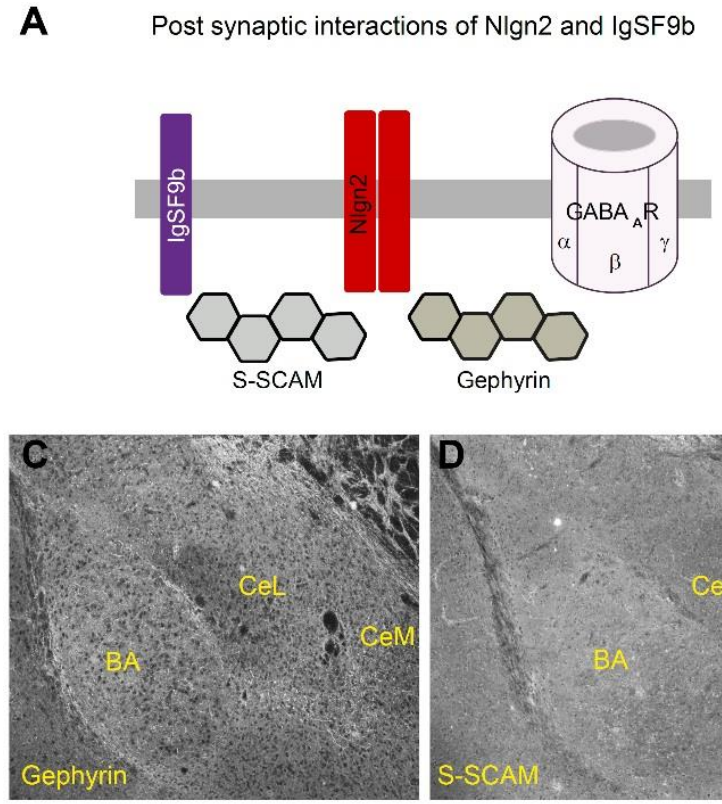
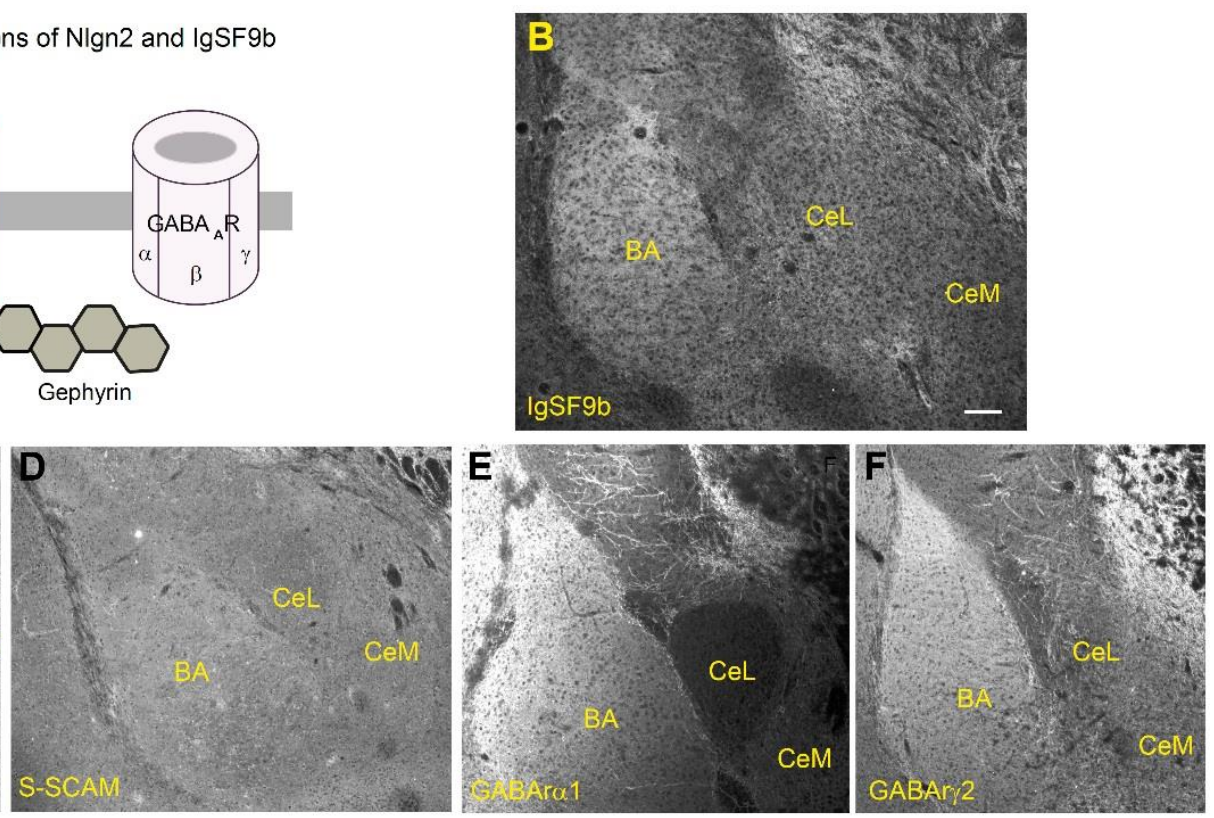

Figure 3.2.13| Amygdala expression of inhibitory post synaptic partners of Nlgn2 and IgSF9b.

A. Schematic representation of known postsynaptic interactions of Nlgn2 and IgSF9b at inhibitory synapses.

B-F. Photomicrographs of expression of IgSF9B (B), gephyrin (C), S-SCAM (D), $\alpha 1$ subunit of GABA receptor (E) and $\gamma 2$ subunit of GABA receptor (F) in amygdala. Scale bar, $200 \mu \mathrm{m}$.

\subsubsection{IgSF9b and Its Post Synaptic Partners are Expressed in Basal and Centromedial Amygdala}

The only established post synaptic partners of IgSF9b are S-SCAM, a scaffold protein that was shown to be expressed at the majority of inhibitory synapses in cell cultures. S-SCAM binds Nlgn2, that in turn binds gephyrin and GABA receptors, and hence bridges IgSF9b and GABA receptors (Figure 3.2.13A) and potentially mediates the effect of IgSF9b deletion on inhibitory synaptic transmission (Woo et al. 2013; Sumita et al. 2007; Poulopoulos et al. 2009). To test this hypothesis, I first showed that IgSF9b, S-SCAM, Nlgn2, gephyrin and two subunits of GABA receptors, $\alpha 1$ and $\gamma 2$ subunit, are expressed in BA and CeM (Figure 3.2.13B-F). 


\section{RESULTS}

A
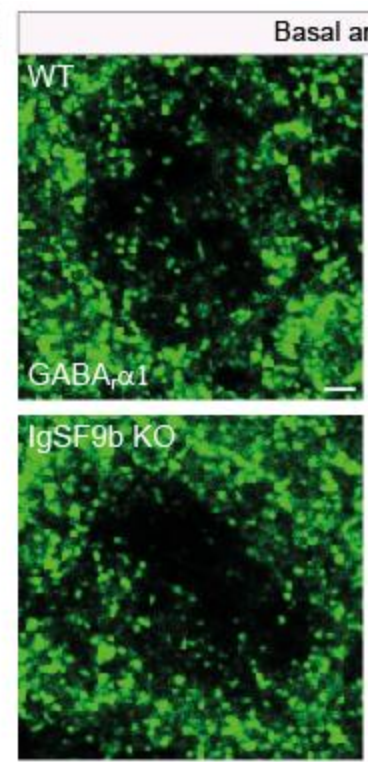

C GABAr $\alpha 1$ particles $/ \mu \mathrm{m}$

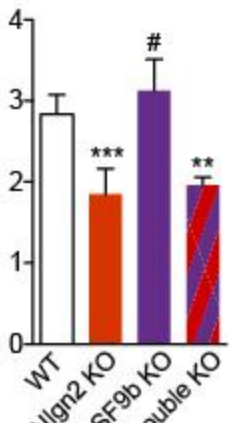

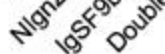

G $\quad \mathrm{GABA}_{r} \gamma 2$ particles $/ \mu \mathrm{m}$

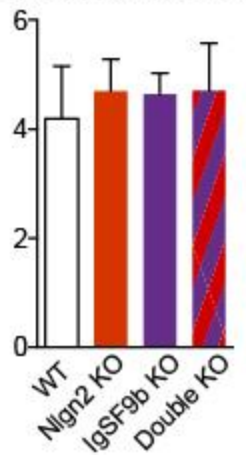

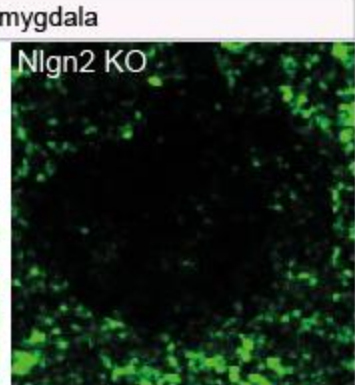

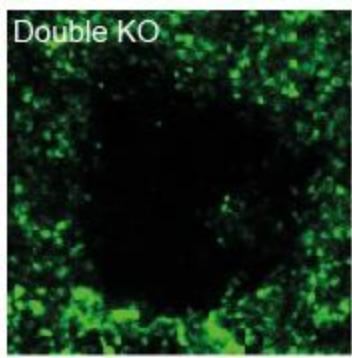

D Size of $G A B A_{r} \alpha 1$ particles (pixels) $\mathbf{E}$

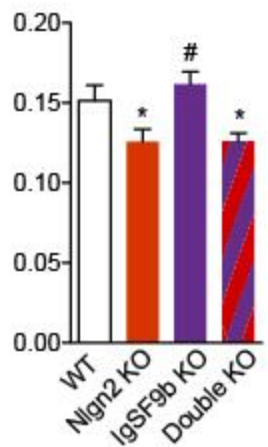

H Size of $\mathrm{GABA}_{r} \gamma^{2}$ particles (pixels)

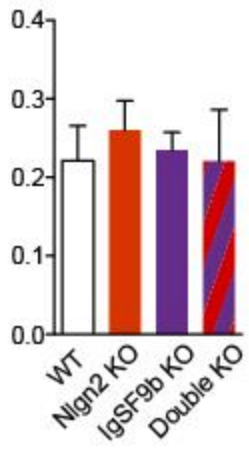

B
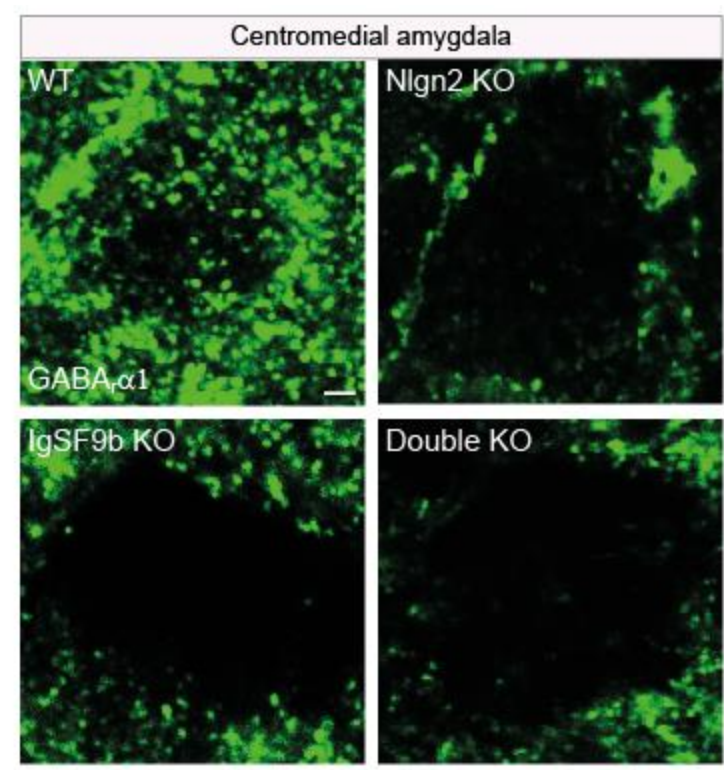

F Size of $\mathrm{GABA}_{\mathrm{r}} \alpha 1$ particles (pixels)
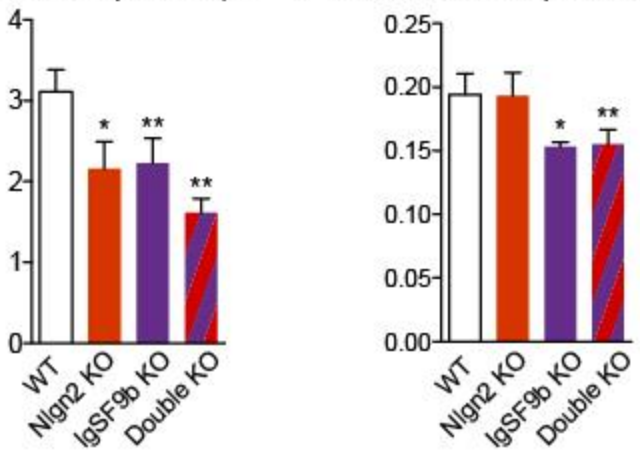

J Size of $\mathrm{GABA}_{\mathrm{r}} \gamma 2$ particles (pixels)

$\mathrm{GABA}_{r} \gamma 2$ particles $/ \mu \mathrm{m}$

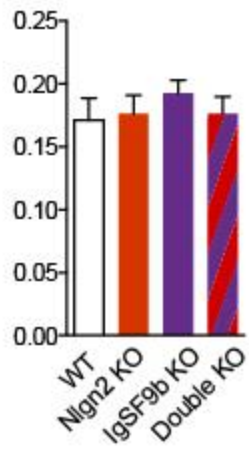

Figure 3.2.14| Effect of Nlgn2 and IgSF9b deletion on composition of perisomatic GABA receptors in basal and centromedial amygdala.

A-B. Photomicrographs showing perisomatic GABAral puncta in basal (A) and centromedial (B) amygdala. Scale bar, $2 \mu \mathrm{m}$. 


\section{RESULTS}

C-F. Density (C, E) and size (D, F) of GABAra1 puncta in WT, Nlgn2 KO, IgSF9b KO and double KOs in basal $(\mathbf{C}, \mathbf{D})$ and centromedial $(\mathbf{E}, \mathbf{F})$ amygdala. Two-way ANOVA: (C) Main effect of Nlgn2 KO, F $\mathrm{F}_{3,21}$ $=13.25, \mathrm{p}=0.0015$, no significant main effect of IgSF9b KO. (D) Main effect of Nlgn2 KO, $\mathrm{F}_{3,21}=13.69$, $\mathrm{p}=0.0013$; no significant main effect of IgSF9b KO. (E) Main effect of Nlgn2 KO, $F_{3,24}=7.73, p=0.0112$, main effect of IgSF9b KO, $\mathrm{F}_{3,24}=6.38, \mathrm{p}=0.0196$. (F) No significant main effect of Nlgn2 KO, main effect of IgSF9b KO, $\mathrm{F}_{3,24}=8.15, \mathrm{p}=0.0095$. No significant interaction between genotypes. Post hoc paired ttests: * p C $0.05, * * \mathrm{p}<0.01, * * * \mathrm{p}<0.001$ compared to WT; \# p < 0.05, \#\# p < 0.01, compared to double KO. $n=5-7(\mathrm{C}, \mathrm{D}), n=6-7(\mathrm{E}, \mathrm{F})$ for each genotype.

G-J. Density (G, I) and size (H, J) of GABAry2 puncta in WT, Nlgn2 KO, IgSF9b KO and double KOs in basal $(\mathbf{G}, \mathbf{H})$ and centromedial (I, J) amygdala. No significant main effect of the genotype. No significant interaction between genotypes. $n=7-8$. All bars represent mean + SEM.

\subsubsection{Nlgn2 KO and IgSF9b KO Reduce Perisomatic GABAr $\alpha 1$ Puncta in Brain Region- Specific Manner}

Next, I investigated whether deletion of Nlgn2 and IgSF9b alters the number and/or composition of GABA receptors in BA and CeM. I quantified the number and size of GABAr $\alpha 1$ and GABAr $\gamma 2$ puncta in Nlgn2 KOs, IgSF9b KOs, double KOs and WTs. I limited my initial analysis to perisomatic sites, as I showed previously that Nlgn2 deletion alters inhibitory synaptic composition specifically at perisomatic sites. In BA, deletion of Nlgn2 robustly reduces the number and size of perisomatic GABAr $\alpha 1$ puncta in Nlgn2 KO and double KO (Figure 3.2.14A, C-D). This finding establishes a molecular mechanism for previously shown reduced inhibitory transmission in BA of Nlgn2 KOs. IgSF9b deletion does not alter GABAr $\alpha 1$ puncta, suggesting that IgSF9b does not regulate GABA receptors in BA at all, or acts at small population of BA neurons that was not assessed in the analysis.

Strikingly, in CeM, IgSF9b deletion reduces the number and size of perisomatic GABAr $\alpha 1$ puncta in IgSF9b KO and in double KO (Figure 3.2.14B, E-F). This finding suggests that IgSF9b regulates inhibitory synaptic composition, and that it does so in brain region- specific manner. Nlgn2 KO reduces the number, but unlike in BA, not the size of GABAr $\alpha 1$ puncta (Figure 3.2.14B, E-F), 

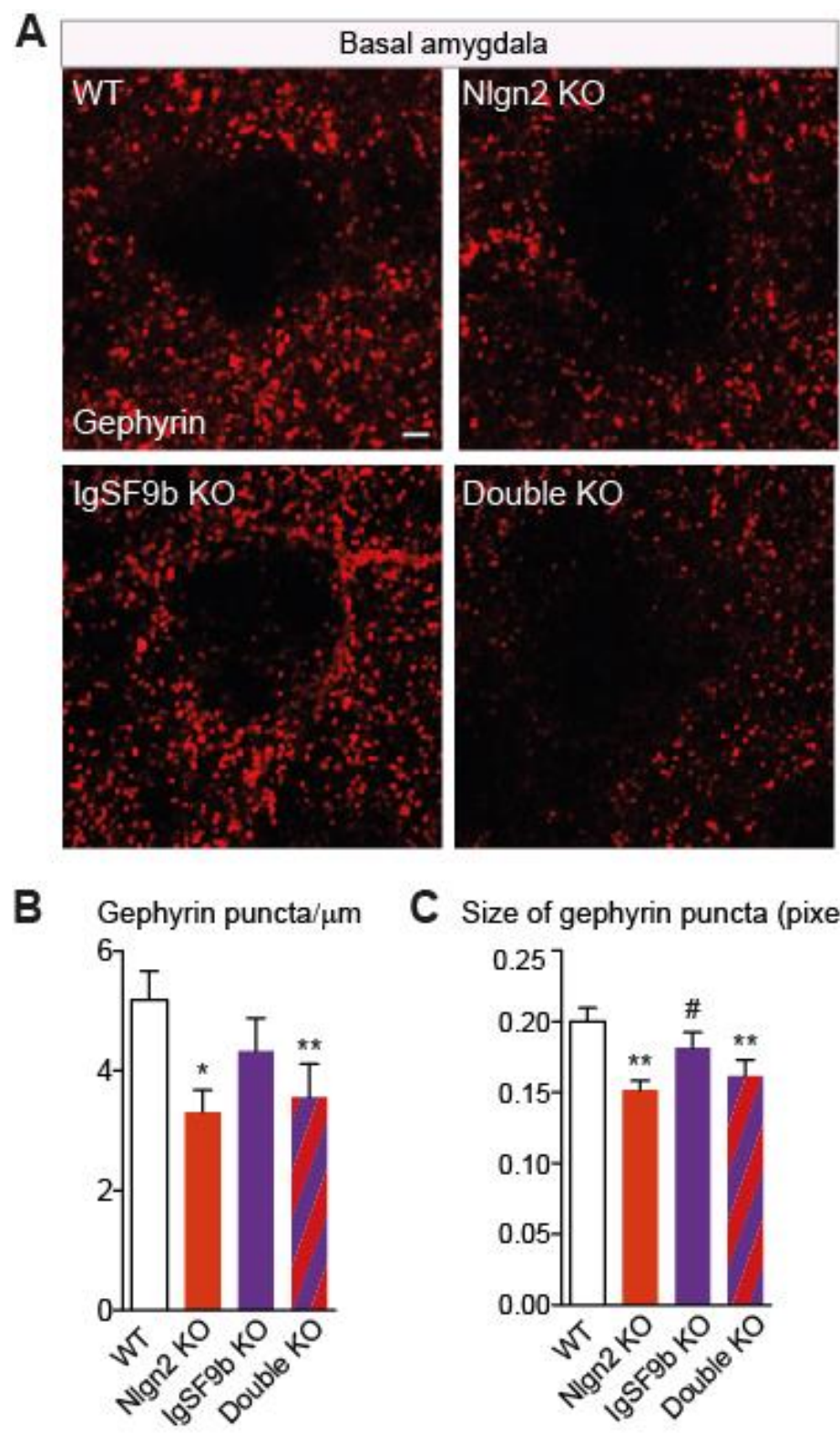

Cize of gephyrin puncta (pixels)

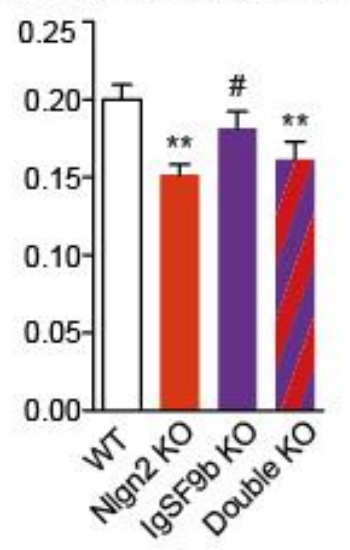

Figure 3.2.15| Effect of Nlgn2 and IgSF9b deletion on perisomatic expression of gephyrin in basal amygdala.

A. Photomicrographs of perisomatic gephyrin expression in WT, Nlgn2 KO, IgSF9b KO and double KOs. Scale bar, $2 \mu \mathrm{m}$.

B-C. Density (B) and size (C) of gephyrin puncta in basal amygdala. Two-way ANOVA: (B) Main effect of Nlgn2 KO, $F_{3,27}=6.48, p=0.0184$, no significant main effect of IgSF9b KO. (C) Main effect of Nlgn2 $\mathrm{KO}, \mathrm{F}_{3,27}=7.19, \mathrm{p}=0.013$; no significant main effect of IgSF9b KO. No significant interaction between genotypes. Post hoc paired ttests: * p C 0.05, ** p<0.01, compared to WT; \# p $<0.05$ compared to double KO. $n=7$ for each genotype. All bars represent mean + SEM. 


\section{RESULTS}

suggesting that similarly to IgSF9b, function of Nlgn2 at the synapses may vary in distinct brain regions.

Finally, neither Nlgn2 KO nor IgSF9b KO alter the expression of GABAr $\gamma 2$ puncta in BA and $\mathrm{CeM}$ (Figure 3.2.14G-J). As GABAr $\gamma 2$ is thought to be a constitutive subunit of GABA receptors (Günther et al. 1995), this finding suggests that Nlgn2 and IgSF9b stabilize receptors containing specific GABA receptor subunits (e.g. $\alpha 1$ subunit), rather than overall GABA receptors, at the postsynaptic membrane.

\subsubsection{IgSF9b KO Does Not Rescue the Deficit in Gephyrin Puncta in Basal Amygdala of Double KOs}

Next, I investigated the mechanism that underlies reduction of perisomatic GABAr $\alpha 1$ puncta in BA of Nlgn2 KOs and double KOs. Nlgn2 drives clustering of synaptic GABA receptors through interaction with scaffold protein gephyrin (Poulopoulos et al. 2009) and I previously showed that Nlgn2 deletion reduces gephyrin puncta at perisomatic sites in BA of Nlgn2 KOs. This points out that deficit in GABAr $\alpha 1$ puncta in Nlgn2 KOs is caused by loss of gephyrin. To determine whether IgSF9b deletion modulates this mechanism in BA of double KOs, I quantified number and size of perisomatic gephyrin puncta in BA of Nlgn2 KOs, IgSF9b KOs and double KOs. Nlgn2 KO reduces the size and number of gephyrin puncta both in Nlgn2 KOs and double KOs, while IgSF9b deletion does not alter gephyrin puncta (Figure 3.2.15A-C). This finding demonstrated that loss of gephyrin correlates with the reduction of GABAr $\alpha 1$ in BA in Nlgn2 KOs and double KOs and supports previous conclusion that IgSF9b does not modulate the composition of inhibitory synapses in BA.

\subsubsection{Nlgn2 KO and IgSF9b KO Do Not Alter Gephyrin and S-SCAM Puncta in Centromedial Amygdala}

Finally, I tested whether loss of gephyrin may explain the reduction in perisomatic GABAr $\alpha 1$ puncta in CeM of Nlgn2 KOs, IgSF9b KOs and double KOs. Analysis of perisomatic gephyrin puncta in CeM revealed that IgSF9b deletion and, surprisingly, Nlgn2 deletion does not reduce the 


\section{Centromedial amygala}

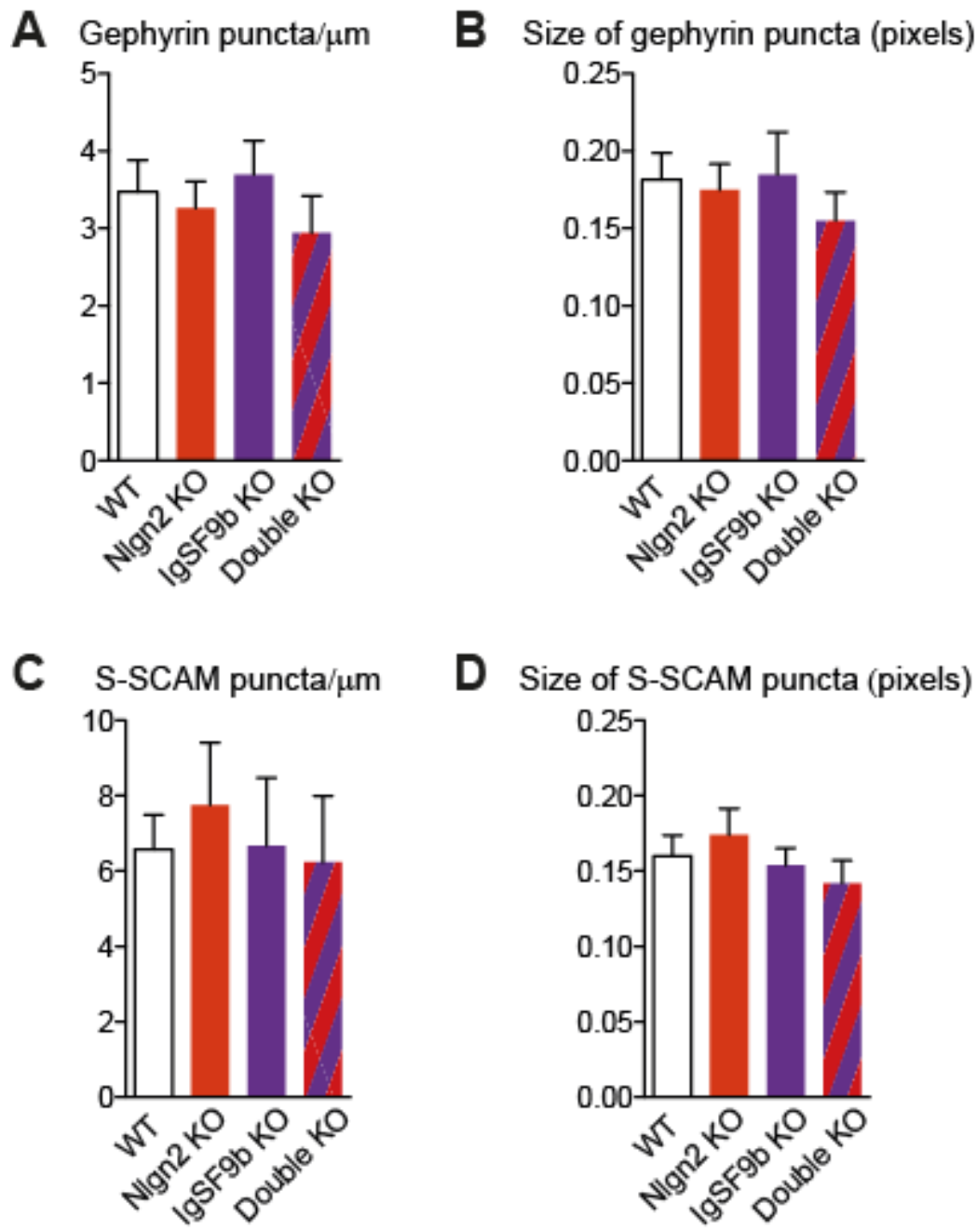

Figure 3.2.16| Effect of Nlgn2 and IgSF9b deletion on perisomatic expression of post synaptic scaffold proteins in centromedial amygdala.

A-B. Density (A) and size (B) of gephyrin puncta in centromedial amygdala in WT, Nlgn2 KO, IgSF9b $\mathrm{KO}$ and double KOs. No significant main effect of genotype. No significant interaction between genotypes. $n=6$ for each genotype.

C-D. Density (C) and size (D) of S-SCAM puncta in centromedial amygdala in WT, Nlgn2 KO, IgSF9b $\mathrm{KO}$ and double KOs. No significant main effect of genotype. No significant interaction between genotypes. $n=5$ for each genotype. All bars represent mean + SEM. 


\section{RESULTS}

number and the size of gephyrin puncta in CeM (Figure 3.2.16A-B). This suggests that Nlgn2 regulates composition of GABA receptors by more than one mechanism, and that this mechanism depends on the brain region.

As both Nlgn2 and IgSF9b bind to scaffold protein S-SCAM at inhibitory postsynaptic sites (Woo et al. 2013), S-SCAM is another possible candidate to mediate the effect of Nlgn2 deletion and IgSF9b deletion on GABAra1 puncta in CeM. To test this, I analyzed perisomatic S-SCAM expression in CeM, but found no significant difference in number and size of S-SCAM puncta in any of the genotypes (Figure 3.2.16 C-D). Therefore, Nlgn2 and IgSF9b regulate the expression of GABAra1 puncta in CeM by a mechanism that does not require loss of gephyrin and S-SCAM from a postsynaptic membrane.

\subsubsection{Nlgn2 KO and IgSF9b KO Perturb the Composition of Specifically Perisomatic} Synapses

To determine whether the effect of $\mathrm{Nlgn} 2 \mathrm{KO}$ and/or IgSF9b KO on composition of inhibitory synapses is specific to perisomatic sites, I quantified the overall intensity of GABAra1 puncta, GABAr $\gamma 2$ puncta and gephyrin puncta in BA, and in addition, S-SCAM puncta in CeM (Figure 3.2.17A-C). There is no difference in overall expression of any of those proteins across genotypes in BA (Figure 3.2.17E-I) and in CeM (Figure 3.2.17G-K), confirming that Nlgn2 and IgSF9b regulate synaptic composition exclusively at perisomatic sites. Interestingly, GABAral puncta appear to be particularly dense in the areas surrounding the cell bodies compared to GABAr $\gamma 2$ puncta (Figure 3.2.17 C-D), suggesting that distinct subunits of GABA receptors may be differentially enriched in perisomatic domains.

\subsubsection{Nlgn2 KO and IgSF9b KO Do Not Alter the Overall Number of Inhibitory and Excitatory Synapses in Basal Amygdala}

Nlgn2 was shown to regulate synaptic formation in cultures (Graf et al. 2004), but synaptogenic activity of Nlgn2 or IgSF9b in BA was never studied. Therefore, I investigated whether Nlgn2 KO or IgSF9b KO reduces the number of inhibitory and excitatory synapses at perisomatic sites in BA 

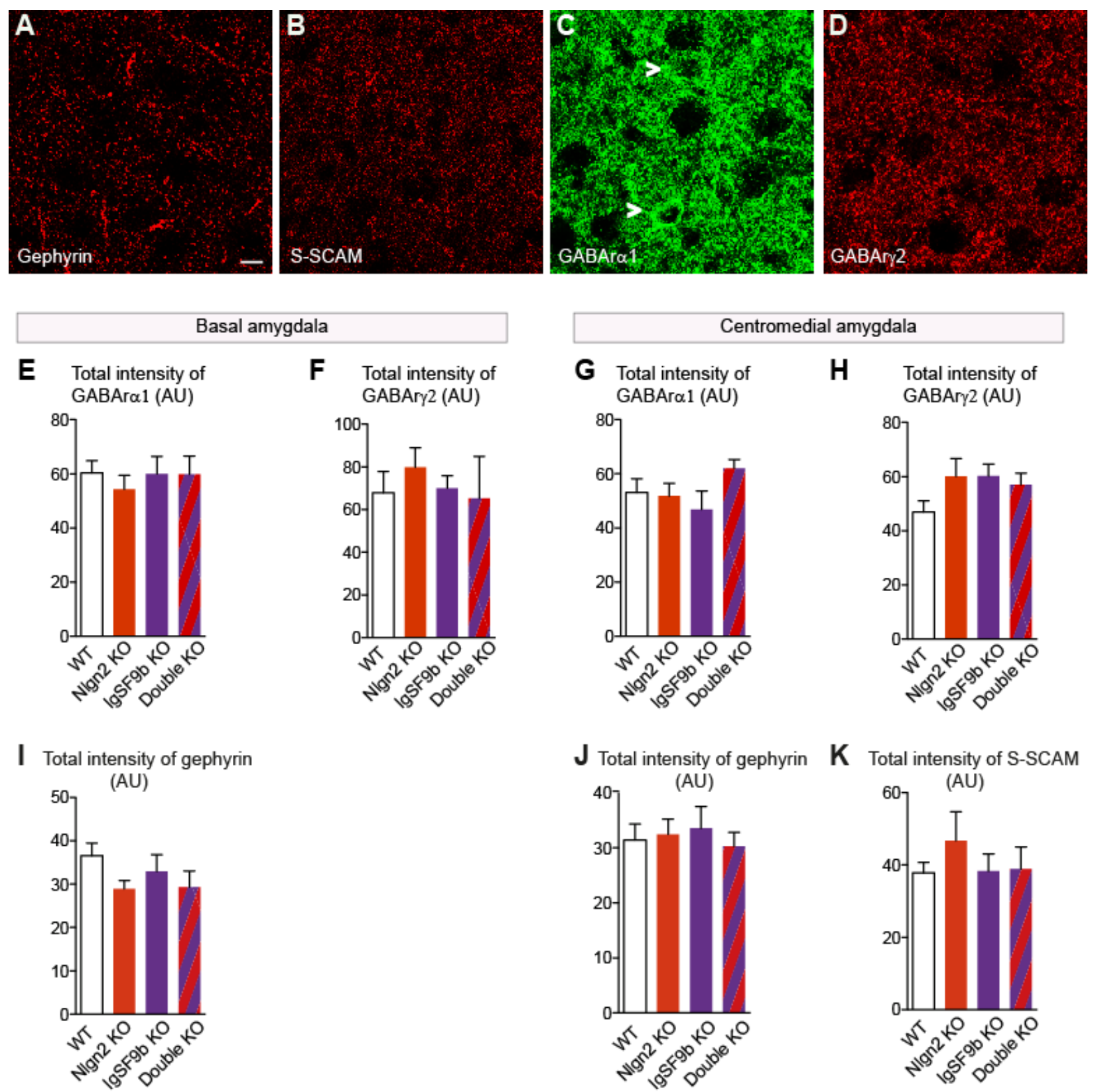

Figure 3.2.17| Overall expression of post synaptic partners of Nlgn2 and IgSF9b in Nlgn2 KO, IgSF9b KO and double KO mice.

A-D. Photomicrographs of gephyrin (A), S-SCAM (B), GABAra1 (C) and GABAr 2 (D) staining from which the total intensity of staining was assessed. White arrows indicate cell bodies with dense perisomatic GABAra1 staining. Scale bar, $10 \mu \mathrm{m}$. All bars represent mean + SEM.

(E-F, I). Total intensity of GABAr $\alpha 1$ (E), GABAr 2 (F) and gephyrin (I) staining in basal amygdala. No significant effect of genotype. No significant interaction between genotypes. $n=5$ (E, F), $n=7$ (I) for each genotype. 


\section{RESULTS}

(G-K). Total intensity of GABAra1 (G), GABAry2 (H), gephyrin (J) and S-SCAM (K) staining in centromedial amygdala. No significant effect of genotype. No significant interaction between genotypes. $n$ $=6(\mathrm{G}, \mathrm{J}), n=7(\mathrm{H}), n=5(\mathrm{~K})$ for each genotype.
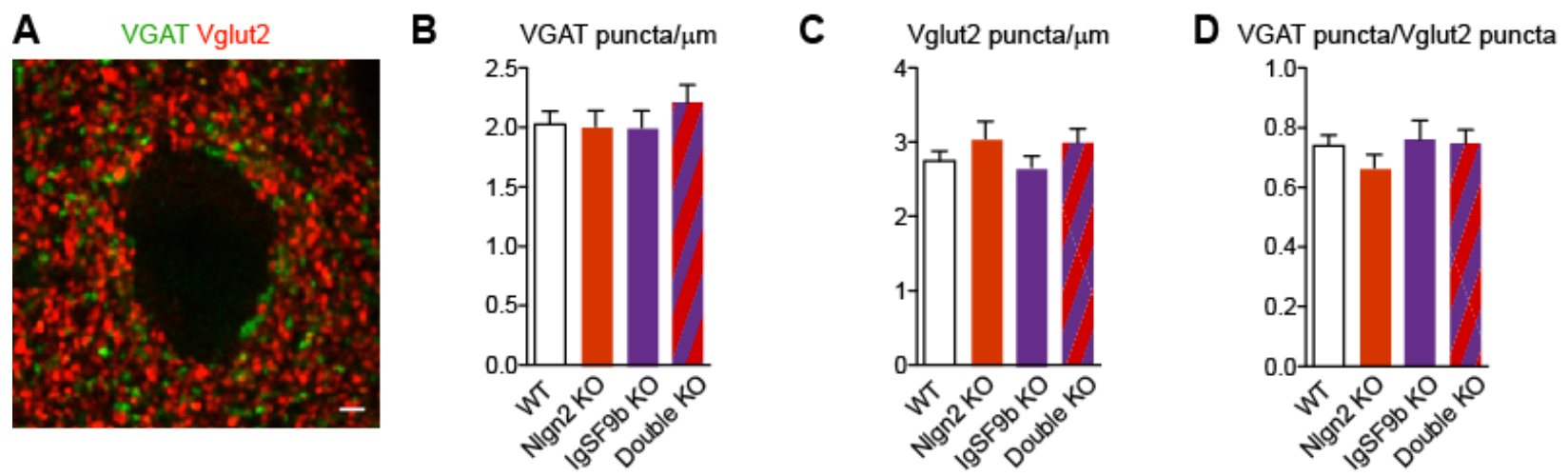

Figure 3.2.18| Total number of perisomatic synapses and VGAT/Vglut2 ratio in basal amygdala of Nlgn2 KO, IgSF9b KO and double KOs.

A. Photomicrograph of VGAT and Vglut2 puncta surrounding the neuron body in basal amygdala. Scale bar, $2 \mu \mathrm{m}$.

B-D. Density of VGAT puncta (B), Vglut2 puncta (C) and VGAT/Vglut2 ratio (D) in WT, Nlgn2 KO, IgSF9b KO and double KOs. No significant effect of genotype. No significant interaction between genotypes. $n=7$ for each genotype. All bars represent mean + SEM.

and/or alters the ratio between inhibitory and excitatory synapses. To that end, I labelled the inhibitory synapses with VGAT and the excitatory synapses with Vglut2 (Figure 3.2.18A), quantified the number of perisomatic VGAT and Vglut2 puncta and calculated VGAT/Vglut2 ratio. I observed no change in number of VGAT puncta, Vglut2 puncta and VGAT/Vglut2 ratio across the genotypes (Figure 3.2.18B-D).

\subsubsection{Nlgn2 KO Impairs Inhibitory Synaptic Transmission in Basal Amygdala}

I showed previously that Nlgn2 KO reduces the frequency of mini inhibitory post-synaptic currents (mIPSCs) in BA. To investigate whether IgSF9b KO modulates this inhibitory deficit in double $\mathrm{KO}$ and/or otherwise alters inhibitory transmission in BA, I measured miniature inhibitory 


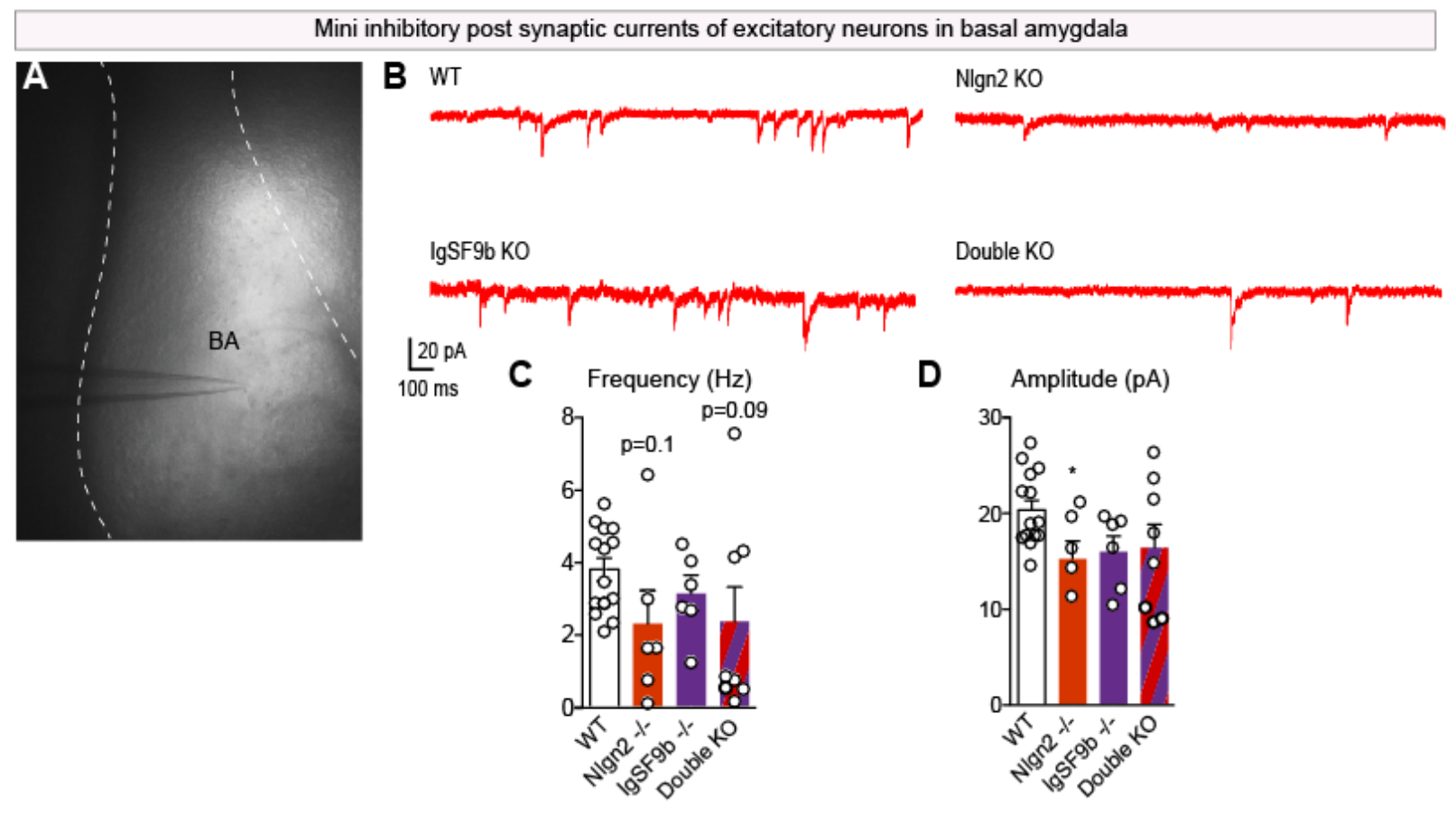

Figure 3.2.19| Effect of Nlgn2 KO and IgSF9b KO on inhibitory synaptic transmission in basal amygdala.

A-B. Low magnification photomicrograph showing the location of recordings (A) and sample traces from basal amygdala of WT, Nlgn2 KO, IgSF9b and double KO (B).

C-D. Average frequency $(\mathbf{C})$ and amplitude (D) of mIPSCs in excitatory neurons of basal amygdala. $n=$ 14 (WT), $n=6$ (Nlgn2 KO, IgSF9b KO) and $n=9$ (double KO). Unpaired ttest: $*$ p $<0.05$ compared to WT. All bars represent mean + SEM.

postsynaptic currents (mIPSCs) in the excitatory neurons in BA of Nlgn2 KOs, IgSF9b KOs and double KOs. Additional data should be collected to reach a statistical significance, however a reduction in mIPSC frequency is readily observed in BA of Nlgn2 $\mathrm{KO}$ mice and double KOs, but not in IgSF9b KO (Figure 3.2.19B-C). This suggests that IgSF9b KO does not rescue the major inhibitory deficit caused by Nlgn2 KO in BA, consistent with lack of effect of IgSF9b KO on inhibitory synaptic composition in BA. Nlgn2 $\mathrm{KO}$ also significantly reduces the amplitude of mIPSCS, but this reduction is less pronounced in double KOs, suggesting that IgSF9b KO may modulate this effect in Nlgn2 KO (Figure 3.2.19D). Interestingly, mIPSC kinetics were significantly altered in IgSF9b KO (rise time: WT $=488.52 \mu \mathrm{s}$, IgSF9b KO $=720.34 \mu \mathrm{s}, \mathrm{p}=<$ 0.0001; decay time: WT $=10.31 \mathrm{~ms}$, IgSF9b KO = $13.33 \mathrm{~ms}, \mathrm{p}=0.0025)$, indicating that IgSF9b may indeed subtly regulate the inhibitory transmission in BA. 


\section{RESULTS}

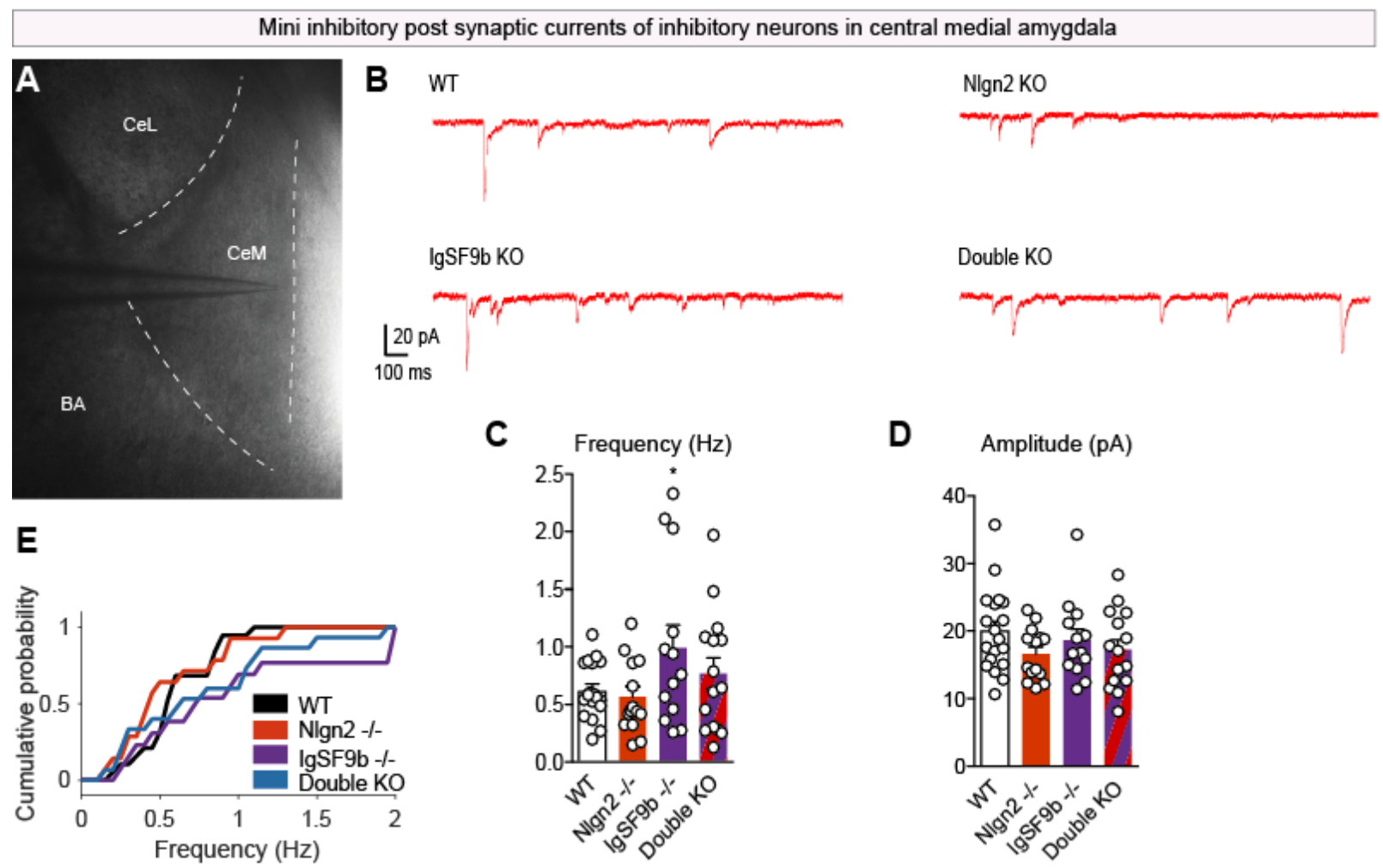

Figure 3.2.20| Effect of Nlgn2 KO and IgSF9b KO on inhibitory synaptic transmission in centromedial amygdala.

A-B. Low magnification photomicrograph showing the location of recordings (A) and sample traces from centromedial amygdala of WT, Nlgn2 KO, IgSF9b and double KO (B).

C-D. Average frequency (C) and amplitude (D) of mIPSCs of inhibitory neurons in centromedial amygdala. Two-way ANOVA: (C) Main effect of Nlgn2 KO, $\mathrm{F}_{3,59}=1.24$, p = 0.271, main effect of IgSF9b KO, F $\mathrm{F}_{3,59}$ $=5.45, \mathrm{p}=0.023$. No significant interaction between genotypes. Post hoc paired ttests: $* \mathrm{p}<0.05$, compared to WT. $n=16$ (WT), $n=13$ (Nlgn2 KO), $n=12$ (IgSF9b KO) and $n=14$ (double KO). All bars represent mean + SEM

E. Cumulative probability plot of mIPSCs frequencies. Kolmogorov- Smirnov statistical analysis: IgSF9b vs WT, p < 0.0001; double KO vs WT, p < 0.0001; Nlgn2 KO vs Double KO, p < 0.05; Nlgn2 vs WT, not significant. 


\subsubsection{IgSF9b KO Enhances Inhibitory Synaptic Transmission in Centromedial Amygdala of Nlgn2 KOs}

Next, I investigated the effect of Nlgn2 KO and IgSF9b KO on inhibitory synaptic transmission in CeM. Mesuring mIPSCS in CeM revealed that IgSF9b KO increases the frequency of mIPSCS compared to WT, while Nlgn2 KO does not affect the frequency and amplitude of mIPSCS (Figure 3.2.20B-C). While in double KO the mean frequency of mIPSCs is not significantly different from WT, analyzing the frequency values distribution revealed a significant shift towards higher values in IgSF9b KO and double KO (Figure 3.2.20E). No significant change in kinetics of mIPSCs was observed across genotypes. Interestingly, the kinetics were slower compared to BA (rise time: $\mathrm{BA}=488.52 \mu \mathrm{s}, \mathrm{CeM}=978 \mu \mathrm{s}$; decay time: $\mathrm{BA}=10.31 \mathrm{~ms}, \mathrm{CeM}=15.1 \mathrm{~ms}$ for WT). Combined with results obtained from $\mathrm{BA}$, these findings demonstrate that IgSF9b modulates inhibitory transmission predominantly in $\mathrm{CeM}$ and not in BA. Moreover, IgSF9b KO enhances the inhibitory transmission in CeM of Nlgn2 KOs, indicating that IgSF9b and Nlgn2 may interact in CeM in a way that leads to reversal of CeM overactivation in double KOs.

\subsubsection{IgSF9b may be a novel regulator of inhibitory synapses onto inhibitory neurons}

What cellular mechanism may underlie the striking CeM- specificity of IgSF9b function? To begin to address this question, I compared the neuronal composition of BA vs CeM by taking advantage of transgenic mice in which neurons expressing inhibitory marker VGAT are labelled with GFP. BA is a cortical-like structure and as expected, only a small population of BA neurons $(\sim 20 \%)$ is inhibitory. In stark contrast, the majority of CeM neurons are inhibitory (Figure 3.2.21A), indicating that almost all the inhibitory post synaptic sites in CeM belong to inhibitory neurons. Therefore, the specificity of IgSF9b function may be explained in terms of specificity towards inhibitory synapses onto inhibitory neurons, positioning IgSF9b as potentially the first known synaptic organizer of specific subpopulation of inhibitory synapses.

Finally, I investigated whether the neuron population in CeM is homogeneous by labelling CeM with inhibitory markers PV, SOM, calretinin and calbindin. PV, SOM and calretinin were all expressed at very low levels in CeM (images not shown) and calbindin labeled $\sim 60 \%$ of neurons 


\section{RESULTS}
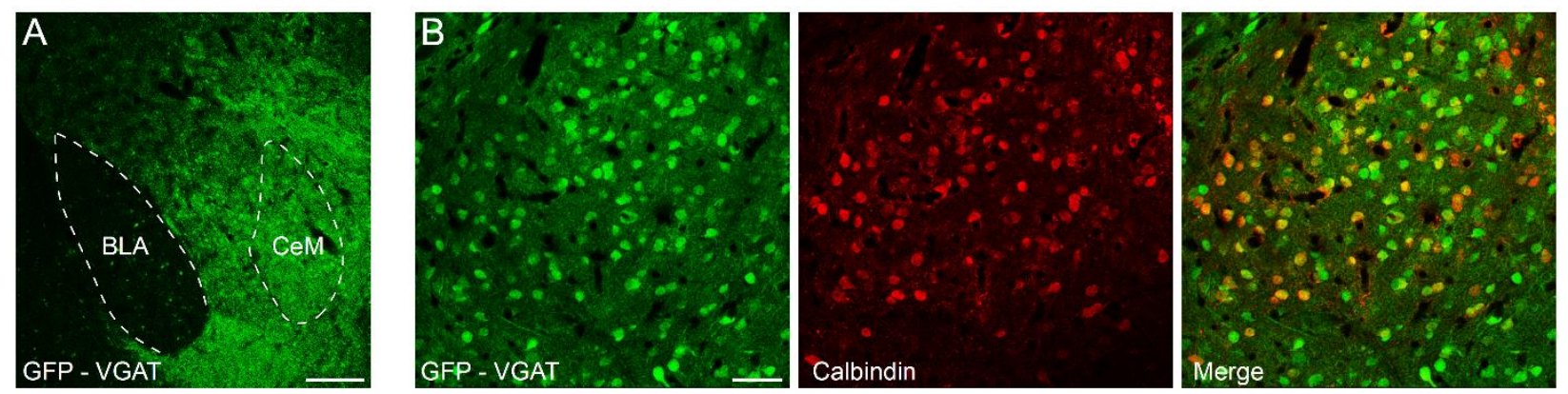

Figure 3.2.21| Expression of VGAT and Calbindin in centromedial nucleus.

A. Photomicrograph showing GFP expression in basal and centromedial amygdala of mouse in which VGAT expressing neurons are tagged with GFP (Venus- Vgat mouse). Scale bar, $200 \mu \mathrm{m}$.

B. Colocalization of GFP and inhibitory marker Calbindin in centromedial nucleus of Venus- Vgat mouse. Scale bar, $50 \mu \mathrm{M}$.

in CeM (Figure 3.2.21B). This suggests that there are at least two populations of inhibitory neurons in $\mathrm{CeM}$ that differentially express calbindin. Future studies will show whether IgSF9b functional specificity depends not only on the type of the synapse but also on the type of inhibitory neuron.

\subsubsection{Summary and Conclusions - Part 2}

The goal of the present study was to describe the cellular and molecular mechanism by which IgSF9b KO rescues the activity enhancement of CeM during anxiety processing in Nlgn2 KOs. To this end, I characterized the expression pattern of IgSF9b in the brain and showed that IgSF9b is expressed in most brain regions and is localized at subsynaptic domain at the periphery of inhibitory synapses. Next, I focused on four possible common synaptic partners of both IgSF9b and Nlgn2, and tested whether their expression levels in BA and CeM are altered by Nlgn2 $\mathrm{KO}$ and/or IgSF9b KO. In addition, I quantified the number of inhibitory and excitatory synapses in basal amygdala. The results of molecular characterization of Nlgn2 and IgSF9b KO mice are summarized in Table 3.2.1. 
RESULTS

Table 3.2.1| Effect of Nlgn2 KO and IgSF9b KO on molecular composition of inhibitory synapses in basal and central amygdala.

Nlgn2 KO

BA

CeM
IgSF9b KO

BA

CeM

\begin{tabular}{|c|c|c|c|c|c|c|c|c|}
\hline GABAr $\alpha 1$ & $\downarrow \downarrow$ & $\leftrightarrow$ & $\downarrow$ & $\leftrightarrow$ & $\leftrightarrow$ & $\leftrightarrow$ & $\downarrow \downarrow$ & $\leftrightarrow$ \\
\hline GABAr $\gamma 2$ & $\leftrightarrow$ & $\leftrightarrow$ & $\leftrightarrow$ & $\leftrightarrow$ & $\leftrightarrow$ & $\leftrightarrow$ & $\leftrightarrow$ & $\leftrightarrow$ \\
\hline Gephyrin & $\downarrow \downarrow$ & $\leftrightarrow$ & $\leftrightarrow$ & $\leftrightarrow$ & $\leftrightarrow$ & $\leftrightarrow$ & $\leftrightarrow$ & $\leftrightarrow$ \\
\hline S-SCAM & $N A$ & $N A$ & $\leftrightarrow$ & $\leftrightarrow$ & $N A$ & $N A$ & $\leftrightarrow$ & $\leftrightarrow$ \\
\hline VGAT & $\leftrightarrow$ & $\leftrightarrow$ & $N A$ & $N A$ & $\leftrightarrow$ & $\leftrightarrow$ & $N A$ & $N A$ \\
\hline Vglut2 & $\leftrightarrow$ & $\leftrightarrow$ & $N A$ & $N A$ & $\leftrightarrow$ & $\leftrightarrow$ & $N A$ & $N A$ \\
\hline
\end{tabular}

$\downarrow$ - reduction in number and size of the puncta

$\downarrow$ - reduction in number of the puncta

$N A$ - not available

All the molecular alterations showed in the table above are present in the double KO, suggesting that IgSF9b KO and Nlgn2 KO do not reciprocally modulate each other's effect on composition of inhibitory synapses in BA and CeM. Regardless the lack of molecular interaction, IgSF9b KO alters synaptic inhibitory transmission in CeM of Nlgn2 KO, suggesting that Nlgn2 and IgSF9b may interact on cellular level specifically at CeM.

Together, these findings demonstrate that IgSF9b KO may decrease anxiety-associated activation of $\mathrm{CeM}$ in Nlgn2 $\mathrm{KO}$ by restoring the inhibitory transmission onto the CeM neurons through currently unknown synaptic interaction. As most of the inhibitory synapses in CeM contact inhibitory neurons, my findings raise an intriguing possibility that inhibitory synapses onto inhibitory neurons may be critical for regulation of anxiety processing, thus offering a novel target for pharmacotherapy of anxiety disorders. 


\section{DISCUSSION}

\section{DISCUSSION}

Using a combination of behavioral assessment, quantitative morphological analysis, cFOS induction assay, anatomical tracing and in vivo and in vitro electrophysiology, I show here that 1) Nlgn2 deletion in mice produces deficits in the structure and function of perisomatic inhibitory synapses in the basal amygdala, accompanied by a prominent anxiety phenotype and a corresponding anxiety-associated overactivation of neurons projecting to centromedial amygdala; and 2) deletion of IgSF9b alters the structure and enhances the function of inhibitory synapses in centromedial amygdala, as well as rescuing the anxiety phenotype and the associated overactivation of centromedial amygdala in Nlgn2 KO mice. This is the first comprehensive study of the molecular, cellular and circuitry role of cell adhesion proteins in regulating anxiety behaviors, as well as the first report of neural correlates of anxiety processing in vivo in centromedial amygdala. My findings indicate that Nlgn2 and IgSF9b bidirectionally regulate anxiety-related behaviors by modulating inhibitory transmission in distinct parts of the anxietyprocessing circuit. Thus, my work provides important insights both into the molecular mechanisms by which mutations in Nlgn2 and IgSF9b may contribute to psychiatrically relevant phenotypes and into the functional role of amygdala in anxiety processing.

\subsection{Consequences of Nlgn2 and IgSF9b Deletion on Anxiety- Related Behavior in Mice}

\subsubsection{Consequences of Nlgn2 Deletion on Anxiety- Related Behavior}

As reported previously (Blundell et al. 2009), deletion of Nlgn2 leads to robust anxiety phenotype that I confirmed in a battery of three well validated anxiety assays: elevated plus- maze (EPM), open field test (OFT) and light- dark box (LD). These tests exploit the natural aversion of mice towards elevated, exposed and brightly lit areas (Rodgers \& Dalvi 1997; Carola et al. 2002) and are the tests of choice for evaluating unconditioned anxiety-related behaviors.

Since EPM, OFT and LD require novelty-induced exploration of neutral and aversive compartments of the test chambers, non- anxiety related alterations in locomotor activity of the mice may confound their anxiety phenotype. Although I found that Nlgn2 KO mice showed 


\section{DISCUSSION}

reduced locomotor activity during anxiety testing as previously reported (Wöhr et al. 2014), correction of activity in anxiogenic compartments for overall activity levels did not reduce the robustness of anxiety phenotype of Nlgn2 $\mathrm{KO}$ mice (Figure 3.1.1). Moreover, I confirmed that the reduced locomotor activity of Nlgn2 KO mice during anxiety assessment is due to noveltyinduced enhancement of freezing behavior and not due to a primary locomotor impairment. Therefore, the reported reduction of exploratory activity in Nlgn2 KO mice, which was measured under anxiogenic conditions in the OFT (Wöhr et al. 2014), reflects their anxiety phenotype. Nlgn2 KO mice demonstrated strong thigmotaxis during OFT which prompted me to include peripheral areas of the open field chamber, in addition to the customary center zone, in the behavioral analysis (Figure 3.2.2). Taken together, these findings demonstrate that Nlgn2 regulates anxiety- related behavior in mice.

\subsubsection{Consequences of Deletion of IgSF9b in NIgn2 KO mice on Anxiety- Related Behavior}

A decrease in anxiety- related behavior of IgSF9b KO mice was evident in the robust increase in the number of entries and the time spent in the center of the OF. However, while there was a significant increase in novelty- induced exploration in IgSF9b KO mice, the normalized activity in the center of the OF is not significantly different between IgSF9b KO and WT mice, although a trend towards decrease in IgSF9b KO mice could be observed (Figure 3.2.1-3.2.2). Taken together, these findings indicate that deletion of IgSF9b mildly reduces the anxiety- related phenotype. Characterization of the home cage locomotor activity of IgSF9b KO mice will be required to distinguish between a genuine decrease in anxiety behavior and a general increase in locomotor activity.

This is the first report of an anxiety- related phenotype of IgSF9b KO mice, which is strikingly opposite to the phenotype of Nlgn2 KO mice with respect to both anxiety behavior and locomotion during OFT. This indicates that Nlgn2 and IgSF9b may bidirectionally regulate anxiety- related behavior. Complete rescue of the anxiety related phenotype in double Nlgn 2 x IgSF9b KO mice further validates the important role these proteins may have in regulation of anxiety, thus inspiring a detailed characterization of individual and collective functions of Nlgn2 and IgSF9b in the anxiety- processing circuit. 


\section{DISCUSSION}

\subsection{Consequences of Nlgn2 Deletion on Synaptic Structure and Synaptic Function in the Anxiety- Processing Circuit}

\subsubsection{Effect of Nlgn2 Deletion on Inhibitory Synapses in Basal Amygdala}

Consistent with previous reports on dissociated neuronal cultures and retina (Varoqueaux et al. 2004; Hoon et al. 2009; Poulopoulos et al. 2009) I show that Nlgn2 is expressed almost exclusively at inhibitory synapses in basal amygdala (BA) of adult mice. In line with findings in hippocampus of Nlgn2 KO mice, loss of Nlgn2 leads to reduction in gephyrin in BA (Figure 3.2.15). Moreover, to my knowledge, this is the first report showing that deletion of Nlgn2 leads to a robust loss of the $\alpha 1$ subunit of $\mathrm{GABA}_{\mathrm{A}}$ receptor $(\mathrm{GABAr} \alpha 1)$ in the brain (Figure 3.2.14), thus expanding the repertoire of Nlgn2 postsynaptic core interactions that so far included gephyrin, collybistin and $\gamma 2$ subunit of GABA receptors (Jedlicka et al. 2011; Poulopoulos et al. 2009).

Loss of the GABAra1 subunit was shown to reduce clustering of GABA receptors and impair inhibitory transmission in cerebellum, thalamus, lateral amygdala and hippocampus (Sur et al. 2001; Kralic et al. 2002; Goldstein et al. 2002; Wiltgen et al. 2009). Moreover, a 50\% decrease in mIPSC frequency and a $20 \%$ decrease in mIPSC amplitude was reported in hippocampal pyramidal neurons of GABAra1 KO mice (Goldstein et al. 2002), consistent with the substantial reduction in mIPSC frequency and a small reduction in mIPSC amplitude in excitatory neurons in BA of Nlgn2 KO mice (Figure 3.2.19). The deficit in inhibitory synaptic transmission in Nlgn2 KO mice may therefore be a direct consequence of loss of the GABAr 1 l subunit in BA (Figure 3.2.14).

What is the molecular mechanism underlying the GABArol subunit reduction in BA of Nlgn2 KO mice? The most straightforward explanation is that absence of Nlgn2 reduces the localization of gephyrin at the synaptic plasma membrane, thus preventing gephyrin- mediated clustering of GABA receptors at the postsynaptic sites. In line with this notion, I observed a marked reduction in gephyrin puncta in BA of Nlgn2 KO mice (Figure 3.2.15). However, Nlgn2 was shown to cluster GABA receptors in the absence of gephyrin (Kneussel, Helmut Brandstätter, et al. 2001; Fritschy 


\section{DISCUSSION}

et al. 2006; Dong et al. 2007). Moreover, findings in GABAra2 subunit KO mice show a dramatic loss of gephyrin but preservation of majority of fully functional $\alpha 1$-containing receptors, indicating that incorporation of GABAr 1 subunit into GABA receptors does not require gephyrin (Fritschy et al. 2006). Therefore, the reduction in postsynaptic GABAra1 subunits may point out to a novel role of Nlgn2 in direct clustering of $\alpha 1$ subunit- containing GABA receptors in amygdala. While in the absence of direct evidence of interaction between Nlgn2 and GABAr $\alpha 1$ subunit this explanation remains speculative, the possible gephyrin- independent interaction between $N \operatorname{lgn} 2$ and the $\alpha 1$ subunit may underlie the reduction of $\alpha 1$ receptors in centromedial amygdala (see below).

In contrast to previously reported findings in stratum pyramidale of Nlgn2 KO mice (Poulopoulos et al. 2009), I did not detect a reduction in number and size of GABAry2 subunit clusters in the BA of Nlgn2 KO mice (Figure 3.2.14). The GABAr $\gamma 2$ subunit was postulated to be a constitutive subunit of GABA receptors and to translocate to the neuronal membrane exclusively upon clustering with GABAra subunits (Essrich et al. 1998; Kneussel et al. 1999). Unaltered levels of the $\gamma 2$ subunit would therefore indicate that deletion of Nlgn2 does not lead to loss of overall GABA receptors in $\mathrm{BA}$ and instead alters the composition of GABA receptors e.g. by compensation with GABAr $\alpha 2$ or $\alpha 3$ subunits. By this logic, the resulting receptors must be less efficient than original $\alpha 1$ containing receptors to explain the reduction in inhibitory transmission in BA. An alternative interpretation arises from a study showing that in mice lacking the GABAr 22 subunit, inhibitory transmission is partially preserved and is mediated by GABA receptors containing $\gamma 3$ subunit, disproving the essential role of $\gamma 2$ subunit in GABAergic transmission (Kerti-Szigeti et al. 2014). Based on this finding, it is conceivable that there is a putative subpopulation of GABA receptors in BA which contains $\alpha 1$ and $\gamma 3$ subunits and whose clustering is selectively impaired by Nlgn2 deletion, explaining the unaltered level of GABAr 2 subunit in Nlgn2 KO mice. Lending support to this theory, an immunoreactivity for GABAr 3 subunit was detected in basal amygdala (Herb et al. 1992). Further analysis of expression of GABAr $\gamma 3$ subunit and subunits that were previously shown to compensate for loss of GABAr $\alpha 1$ in amygdala, such as $\alpha 2$ and $\alpha 3$ (Kralic et al. 2002), will clarify whether reduction in $\alpha 1$ subunit 


\section{DISCUSSION}

reflects reduction in number of GABA receptors or subunit composition change. Regardless of the exact mechanism, deletion of Nlgn2 impairs GABAergic transmission in BA.

Interestingly, Nlgn2 deletion in the BA leads to a specific loss of perisomatic inhibitory postsynaptic components. In addition to already reported perisomatic reduction in gephyrin (Jedlicka et al. 2011; Poulopoulos et al. 2009), I show that loss of Nlgn2 reduces GABAra1 puncta strictly at perisomatic synapses (Figure 3.2.17). These findings are particularly interesting in light of my observation that almost all gephyrin-positive structures in BA contain Nlgn2, indicating that the loss of Nlgn2 at non-perisomatic synapses is compensated by other synaptic adhesion molecules such as $\beta$-dystroglycan and Neurofascin (Craig \& Kang 2007; Panzanelli et al. 2011; Burkarth et al. 2007).

What is the possible mechanisms underlying perisomatic reduction of gephyrin in Nlgn2 KO mice? It was previously shown that Nlgn2 is required to target gephyrin to the postsynaptic membrane, as gephyrin forms large cytoplasmic aggregates in absence of binding to Nlgn2 (Poulopoulos et al. 2009). I did not observe an increase in intracellular gephyrin clusters in BA of Nlgn2 KO mice. However, I empirically observed stronger gephyrin labelling of axon initial segments (AIS) in Nlgn2 KO mice compared to WT mice (Figure 4.1). While this finding awaits quantitative verification, it may indicate an intriguing possibility that in absence of Nlgn2, gephyrin is preferentially targeted to synapses at AIS on the expense of the somatic synapses, and that gephyrin recruitment to perisomatic and AIS synapses requires distinct mechanisms. In line with this hypothesis, gephyrin clustering at AIS synapses is dependent on interaction with the adhesion protein Neurofascin (Burkarth et al. 2007; Ogawa \& Rasband 2008). Therefore, Nlgn2 and Neurofascin may be required for perisomatic and AIS targeting of gephyrin, respectively. Interestingly, GABAergic synapses at the AIS of BA projection neurons were recently implicated in regulation of fear- related behavior (Saha et al. 2017). This indicates that the molecular mechanisms balancing the inhibitory input across distinct neuronal compartments, such as described above, may be involved in regulating clinically- relevant behaviors. 


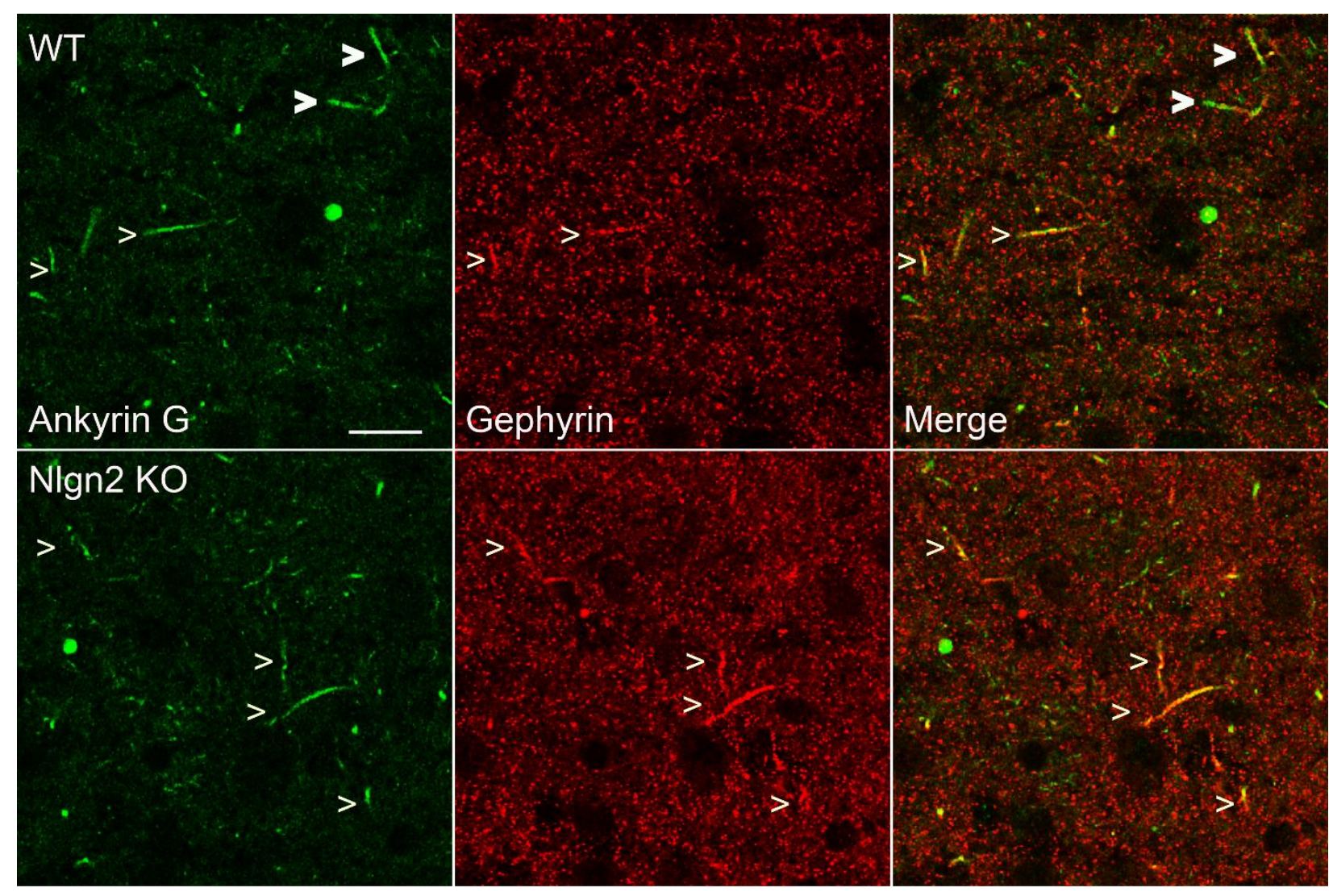

Figure 4.1| Colocalization of Gephyrin and AIS marker Ankyrin G in Nlgn2 KO and WT mice.

Thick arrows indicate AIS that are not colocalized with gephyrin strings in WT (upper panel). In Nlgn2 KO, all AIS colocalize with Ankyrin G strings (lower panel). Scale bar, $20 \mu \mathrm{m}$.

The overall number of inhibitory presynapses remains unaffected by Nlgn2 deletion (Figures 3.1.9, 3.2.18). This finding is in agreement with multiple reports demonstrating that deletion of inhibitory postsynaptic core components including Nlgn2, GABA receptor subunits and gephyrin does not affect the expression of presynaptic markers such as GAD 67 and VGAT (Fritschy et al. 2006; Kneussel et al. 1999; Poulopoulos et al. 2009). One exception to this statement is a study that demonstrated a reduction in VGAT in Nlgn2 $\mathrm{KO}$ mice, however electron microscopy imaging in the same study revealed that the overall number of inhibitory synapses remained unaltered (Blundell et al. 2009). Therefore, it may be concluded that Nlgn2 deletion does not alter the number of inhibitory contacts in vivo. 


\section{DISCUSSION}

The strictly perisomatic effect of Nlgn2 deletion is consistent with the observation that loss of Nlgn2 in the cerebral cortex specifically affects synaptic connections made by axons of PVpositive interneurons (Gibson et al., 2009), the primary source of perisomatic inhibition in many brain regions, including BA (Muller et al., 2006; Wolff et al., 2014). Interestingly, I observed a slight increase in the total number of PV-positive interneurons but no change in the number of presynaptic PV-positive terminals, indicating that pruning of the excess inputs may occur during early postnatal development (Figure 3.1.9, 3.1.11). Additionally, Cholecystokinin (CCK) expressing interneurons were shown to form perisomatic synapses onto excitatory neurons in BA (Mascagni \& McDonald 2003). Interestingly, although both GABAr $\alpha 1$ and $\alpha 2$ subunits are strongly expressed in BA, post synaptic sites targeted by CCK-positive interneurons in BA contain predominantly GABAr $\alpha 1$ subunit (Marowsky et al. 2004). Loss of $\alpha 1$ clusters in Nlgn2 KO mice may therefore reflect the effect of Nlgn2 deletion on CCK- positive terminals onto excitatory neurons in BA, in addition to a well-documented effect of Nlgn2 deletion on PV-positive terminals.

In agreement with a key role for Nlgn2 at perisomatic inhibitory synapses in BA, I observed that Nlgn2 deletion results in a pronounced reduction in the frequency and a slight, but significant, reduction in the amplitude of mIPSCs of excitatory neurons in BA (Figure 3.2.19). The observed reduction in the amplitude is contradictory to the findings of our collaborators Paolo Botta and Andreas Luthi (Friedrich Miescher Insitute of Biomedical Research in Basel, Switzerland) and may arise from variability in experimental conditions (Figure 3.1.10). Since a reduction in the mIPSC amplitude reflects a decrease in the number of GABA receptors and a reduction in the mIPSC frequency may reflect decrease in the number of functional postsynaptic sites (Otis et al. 1994; De Koninck \& Mody 1994), my findings are consistent with the molecular alterations of inhibitory synapses in Nlgn2 KO mice described above. Alternatively, the reduction in mIPSC frequency may point to a change in presynaptic release probability. While the unaltered expression of pre-synaptic markers argues against this scenario (Figure 3.2.18), a functional impairment of the vesicle release machinery in Nlgn2 KO mice currently cannot be ruled out and will be addressed in future experiments. 


\section{DISCUSSION}

Taken together, my findings indicate that Nlgn2 deletion affects the postsynaptic structure and transmission of inhibitory perisomatic synapses onto excitatory neurons in BA. The mechanism by which deletion of Nlgn2 would affect only perisomatic synapses is currently unknown, but may potentially be explained by the existence of a molecularly heterogeneous synapse population, in which Nlgn2 is either redundant or not present in some synapses. Further studies will be required to identify the exact molecular mechanisms underlying the function of Nlgn2 in regulating the number and/or composition of GABA receptors. As insufficient GABAergic inhibition in BA is one of the key mechanisms underlying anxiety- related disorders (Sanders \& Shekhar 1995; Gauthier \& Nuss 2015), my findings place Nlgn2 as a regulator of anxiety- related processing.

\subsubsection{Effect of Nlgn2 Deletion on Inhibitory Synapses in Centromedial Amygdala}

In stark contrast to its robust effect on inhibitory synapses in BA, the only consequence of Nlgn2 deletion in centromedial amygdala $(\mathrm{CeM})$ is the reduction in number of perisomatic GABAral clusters. The size of alpha1 clusters, the expression of gephyrin, and inhibitory synaptic transmission are unaltered in Nlgn2 KO mice (Figure 3.2.14, 3.2.16). The fact that Nlgn2 deletion can have brain region- specific effects on mIPSCs was illustrated by previous studies, revealing a pronounced decrease in mIPSC frequency and a lesser decrease in amplitude in area CA1 of hippocampus (Poulopoulos et al., 2009), but a decrease in mIPSC amplitude without accompanying changes in frequency in somatosensory cortex (Gibson et al., 2009) and dentate gyrus (Jedlicka et al., 2011). However, a complete lack of effect of Nlgn2 deletion on both mIPSC frequency and amplitude has never been reported so far. Even more surprisingly, my finding indicates that the well- established role of Nlgn2 in synaptic targeting of gephyrin is not universal across the brain, further highlighting the functional heterogeneity of Nlgn2.

Unlike in BA, inhibitory transmission in CeM is mediated predominantly by $\alpha 2$ subunit containing GABA receptors (Ortinski et al. 2004; Marowsky et al. 2004). Interestingly, $\alpha 2$ subunit containing receptors were reported to demonstrate slower kinetics of mIPSCs compared to $\alpha 1$ containing GABA receptors (Dixon et al. 2014), and accordingly, I observed slower rise and decay times of mIPSCs in CeM compared to BA. Based on this notion, one possible explanation for the lack of consequences of Nlgn2 deletion on inhibitory synaptic transmission in CeM is that Nlgn2 may be 


\section{DISCUSSION}

not involved in clustering of $\alpha 2$ subunit containing GABA receptors. Several lines of evidence may support this hypothesis. I observed that Nlgn2 deletion has no effect on number and size of GABAr 22 clusters in BA in a small sample of mice $(\mathrm{n}=3$ for each genotype, mean value for WT, 2.07; mean value for Nlgn2 KO, 1.74; $\mathrm{p}=0.56$ ), despite affecting gephyrin and GABAr $\alpha 1$ clustering in this region. Moreover, clustering of $\alpha 2$ subunit requires gephyrin (Tretter et al. 2008) which, as I show here, is not affected by Nlgn2 deletion in CeM (Figure 3.2.16). Finally, $\alpha 2$ subunit is highly expressed in lateral part of CeA (Marowsky et al. 2004), while I observed that Nlgn2 is almost completely absent from the lateral part of $\mathrm{CeA}$ (Figure 3.1.7). Taken together, this may possibly indicate that Nlgn2 is not associated with regulation of clustering of the GABAr $\alpha 2$ subunit, and therefore does not affect the inhibitory transmission in CeM. Quantification of GABAra2 subunit in CeM and subunit- specific pharmacological manipulations during patch clamp recording will be necessary to further test this hypothesis.

\subsection{Consequences of IgSF9b Deletion on Synaptic Structure and Synaptic Function in Anxiety- Processing Circuit}

\subsubsection{Subcellular localization of IgSF9b}

Analysis of postsynaptic density components showed tight association of IgSF9b with GABAry2 subunit, indicating that IgSF9b is a synaptic protein (Woo et al. 2013). In dissociated hippocampal cultures, IgSF9b was shown to colocalize with VGAT, gephyrin and Nlgn2, but not with excitatory markers, further supporting its association with inhibitory synapses. Finally, superresolution imaging demonstrated that IgSF9b is localized to a subsynaptic domain at a nanoscale distance from core components of inhibitory synapses (Woo et al. 2013). My findings that IgSF9b clusters 1) have punctuate appearance typical of synaptic clusters; and 2) are closely apposed to $\gamma 2$ subunit (Figure 3.2.12), are in line with the notion that IgSF9b is expressed at the periphery of inhibitory synapses. A similar subsynaptic organization was previously demonstrated for excitatory synapses, where the cell adhesion protein $\mathrm{N}$-cadherin and glutamatergic receptors are localized in two distinct synaptic compartments (Uchida et al. 1996). However, it is important to mention that I did not systematically analyze the distance between IgSF9b clusters and inhibitory 


\section{DISCUSSION}

synaptic markers that were closely associated, but did not colocalize with IgSF9b. Therefore, at this stage, I cannot rule out the possibility that IgSF9b is an extrasynaptic membrane protein.

\subsubsection{Consequences of IgSF9b deletion in basal amygdala}

Deletion of IgSF9b did not affect the synaptic composition and only slightly altered synaptic transmission at inhibitory synapses onto excitatory neurons in BA. While the frequency and the amplitude of mIPSCs were similar between IgSF9b KO and WT mice, there was a significant increase in the rise time and the decay of mIPSCs in IgSF9b KO mice (Figure 3.2.19). This increase may reflect an alteration in the subunit composition of GABA receptors, specifically in alpha subunits which determine the kinetics of the receptor- mediated currents (Vicini et al. 2001).

Mostly unaltered inhibitory transmission onto excitatory neurons in IgSF9b KO mice is consistent with the report that knock-down of IgSF9b predominantly affects inhibitory synapses onto inhibitory neurons. My findings confirm the observation from cell cultures that IgSF9b lacks the ability to cluster gephyrin (Woo et al. 2013), as I observed no deficits in gephyrin expression in IgSF9b KO mice (Figure 3.2.15). Taken together, these findings indicate that IgSF9b is not majorly involved in regulation of inhibition of excitatory neurons in BA.

\subsubsection{Consequences of IgSF9b Deletion in Centromedial Amygdala}

In contrast to BA, I found that loss of IgSF9b leads to reduction in number and size of $\alpha 1$ subunit and to enhancement of inhibitory synaptic transmission in CeM (Figures 3.2.14, 3.2.20). As I and others showed that $\mathrm{CeM}$ contains predominantly inhibitory neurons, this finding indicates that IgSF9b may be a specific regulator of inhibitory transmission onto inhibitory neurons. While knockdown of IgSF9b in cell cultures was shown to reduce the frequency of mIPSCs (Woo et al. 2013), the discrepancy with my results obtained from adult animals may arise from differential makeup of GABAergic receptors during distinct stages of neuronal development (Ortinski et al. 2004). 


\section{DISCUSSION}

While the exact mechanism of upregulation of inhibitory transmission in CeM of IgSF9b KOs is currently unknown, it may involve compensatory upregulation of GABAr $\alpha 2$ and $\alpha 3$ subunits that was previously demonstrated in the forebrain, amygdala and cerebellum of $\alpha 1$ subunit KO mice (Sur et al. 2001; Kralic et al. 2002; Wiltgen et al. 2009). It is important to note that during electrophysiological characterization of Nlgn2 and IgSF9b KO mice I did not rule out that the effect observed mIPSCs frequencies is caused by presynaptic mechanism that affects vesicle release. Further experiments to determine vesicle release probability or the size of readily releasable pool will address the potential contribution of presynaptic mechanisms to inhibitory transmission in Nlgn2 $\mathrm{KO}$ and IgSF9b KO mice.

As I did not observe any alterations in size and number of S-SCAM (Figure 3.2.16), a scaffold protein that was proposed to bridge IgSF9b with core components of inhibitory synapse (Woo et al. 2013), the molecular mechanism underlying loss of $\alpha 1$ in CeM of IgSF9b KO mice is currently unknown. Electron microscopy imaging to determine the precise localization of IgSF9b within neurons might be required to begin to untangle the interactions of IgSF9b underlying its synaptic function. Regardless of the mechanism, deletion of IgSF9b upregulates inhibitory transmission in $\mathrm{CeM}$, which may supress the anxiogenic output of CeM during anxiety- related processing.

\subsection{Synaptic Structure and Synaptic Function in Anxiety- Processing Circuit in Double Nlgn2 IgSF9b KO mice}

Most of the outcomes of Nlgn2 and IgSF9b deletion on synaptic composition and function are present in double KO mice. Accordingly, double KO mice demonstrate a reduction of perisomatic gephyrin and GABAr $\alpha 1$ subunit clusters and strong trend for reduction of mIPSCs frequency in $\mathrm{BA}$, as well as reduction in number and size of $\alpha 1$ subunit clusters and a shift towards highfrequency mIPSCs in CeM (Figures 3.2.14, 3.2.15, 3.2.20). Interestingly, unlike in Nlgn2 KO mice, the amplitude of mIPSCs in BA of double KO mice is not significantly different from the WT mice. However, it should be highlighted that all the data from in vitro electrophysiological experiments in IgSF9b and double KO mice is preliminary and should be further expanded to be conclusive. 


\section{DISCUSSION}

Lack of modulatory effect of IgSF9b deletion on outcomes of Nlgn2 deletion strongly suggests that Nlgn2 and IgSF9b do not interact with each other at the synaptic and cellular level. Therefore, the rescue of anxiety- related behavior in double KO mice must occur at the circuit level, by IgSF9b deletion- conferred alteration of anxiety-associated activity of the networks within and/or across the BA and CeM.

\subsection{Consequences of Nlgn2 and IgSF9b Deletion on Excitatory and Inhibitory Networks in Basal Amygdala}

\subsubsection{Consequences of Nlgn2 deletion on Neurons in BA}

Despite a near-universal presence of Nlgn2 at inhibitory synapses in BA, only CaMKII-positive glutamatergic projection neurons show differential induction of cFOS in Nlgn2 KO mice under anxiogenic conditions. These neurons project to a number of target regions known to mediate anxiety behaviors, including the CeA, hippocampus, bed nucleus of the stria terminalis, and prefrontal cortex (Felix-Ortiz et al. 2013; Namburi et al. 2015; Lebow \& Chen 2016; Sotres-Bayon et al. 2012). Specifically, the glutamatergic projection from BA to CeM was recently implicated in mediation of anxiety- related behavior during exploration of a novel environment (Namburi et al. 2015). In line with this finding, I show that projection neurons in BA that target CeM show enhanced anxiety- associated cFOS induction in Nlgn2 $\mathrm{KO}$ mice (Figure 3.2.6).

In contrast, the complex inhibitory network of local Parvalbumin (PV) - and Somatostatin (SOM) -positive interneurons, which play an essential role in regulating neuronal plasticity and learning in the BA (Wolff et al. 2014; Ehrlich et al. 2009) shows no differential cFOS expression under anxiogenic conditions, even though both types of interneurons receive perisomatic innervation from PV-positive terminals (Muller et al. 2006; A. R. Woodruff \& Sah 2007; Wolff et al. 2014) (Figure 3.2.5). Due to an incompatibility of immunostaining conditions for the Nlgn2 antibody and the PV and SOM antibodies, I cannot rule out the possibility that Nlgn2 may not be expressed in PV- and SOM-positive cells. However, the fact that the vast majority of gephyrin-positive synapses in BA also contain Nlgn2 would argue against this scenario. Alternatively, it is possible that differential compensation by other synaptic adhesion molecules may prevent altered activation of interneurons but not projection neurons, or that under my experimental conditions, acute 


\section{DISCUSSION}

activation of these interneurons does not play a primary role in the anxiety circuitry. Regardless of the mechanism, my data support a model according to which reduced perisomatic inhibition at connections between PV-positive interneurons and CeM targeting projection neurons in BA of Nlgn2 KO mice results in specific overactivation of these projection neurons under anxiogenic conditions, consistent with the exaggerated behavioral anxiety response of Nlgn2 $\mathrm{KO}$ mice.

The notion that synaptic adhesion proteins may function differently at distinct neurons and at distinct synapses, even on the same neuron, has lately received substantial attention. One recent study showed that deletion of Nlgn3 results in enhanced synaptic transmission at inhibitory connections between cholecystokinin (CCK)-positive interneurons and pyramidal cells in area CA1 of hippocampus, without affecting inhibitory connections formed by PV-positive interneurons onto the same pyramidal cells (Földy et al. 2013). Another study showed that deletion of Nlgn4 reduces inhibitory transmission onto excitatory neurons originating specifically from parvalbumin (PV)-positive interneurons in area CA1 in hippocampus (Hammer et al. 2015). These and other findings highlight the fact that the role of adhesion proteins in circuit function cannot be predicted from studies on dissociated cultures or by inference from other brain regions, but must be investigated in the context of specific behaviorally relevant neural circuits

\subsubsection{Consequences of IgSF9b Deletion on Neurons in CeM}

Consistent with my findings that point to a postsynaptic role of IgSF9b specifically in interneurons, PV- positive neurons show differential anxiety- associated cFOS induction in BA of IgSF9b KO mice (Figure 3.2.5). The most parsimonious explanation for a mechanism underlying higher cFOS expression of PV- positive neurons is a deficit in inhibitory tone onto these interneurons. Indeed, knockdown of IgSF9b in cell cultures reduces the frequency of mIPSCs in interneurons (Woo et al. 2013). The proposed deficit in inhibitory transmission in BA may appear contradictory to my findings of increased inhibitory transmission in CeM in IgSF9b KO mice. However, based on my observation, PV-positive cells are virtually absent from CeM. It is therefore plausible that IgSF9b regulates distinct aspects of inhibitory transmission in an interneuron subtype- specific manner. Consistent with this notion, deletion of IgSF9b does not lead to enhanced cFOS induction of SOMpositive interneurons in BA. Due to an incompatibility of staining conditions, I cannot assess the 


\section{DISCUSSION}

possible molecular deficits specifically on PV interneurons, however the mechanism underlying reduced inhibitory tone in BA may involve an uncompensated reduction in $\alpha 1$ subunit of GABA receptors.

It is surprising that enhanced inhibitory tone in BA does not result in reduced cFOS expression of excitatory projectors to $\mathrm{CeM}$ in IgSF9b $\mathrm{KO}$ mice. PV positive interneurons appear to have a complex role in regulation of projection neurons in BA during processing of aversive stimuli. For instance, PV-positive neurons reduced their firing during presentation of electric shock- paired stimulus and showed mixed responses to paw pinch (Bienvenu et al. 2012; Wolff et al. 2014). Here, I show that increased activation of PV-positive neurons does not suppress the excitatory anxiogenic output of BA during anxiety- related processing. Studying the exact contribution of PV- positive neurons to inhibition of anxiety- related excitability of projections neurons in BA, and the role of IgSF9b in regulation of PV-positive neurons may enhance our understanding of molecular mechanisms of anxiety.

\subsubsection{Combined Effect of Nlgn2 and IgSF9b Deletion on Neurons in BA}

The combined effect of IgSF9b and Nlgn2 deletion on activation of excitatory and inhibitory networks in BA of double $\mathrm{KO}$ mice recapitulates the effect of the single $\mathrm{KO}$ of each protein. Accordingly, both excitatory projection neurons, including those that target CeM, and inhibitory $\mathrm{PV}$ - positive interneurons, show high cFOS induction under anxiogenic conditions in double KO mice (Figures 3.2.5, 3.2.6). This finding suggests that the rescue of anxiety- related behavior in double $\mathrm{KO}$ mice does not arise from normalization of anxiogenic BA output, and therefore most probably occurs in downstream targets of BA, with CeM being the most obvious site of the rescue.

Interestingly, the enhancement of PV- positive network is more robust in double $\mathrm{KO}$ than in IgSF9b KO mice. As PV- positive neurons receive excitatory connections from glutamatergic neurons in BA (Woodruff \& Sah 2007), it is plausible that increased overall excitatory drive in Nlgn2 KO mice further enhances the IgSF9b- deletion conferred overactivation of PV-positive neurons. The fact that this robust overactivation of PV-positive network is not accompanied by a suppression of glutamatergic neurons in double KO mice confirms that Nlgn2 deletion impairs 


\section{DISCUSSION}

inhibitory transmission between PV-positive interneurons and glutamatergic neurons, and demonstrates that IgSF9b deletion does not rescue this major outcome of Nlgn2 deletion in BA.

\subsection{Consequences of Nlgn2 and IgSF9b Deletion on Anxiety- Associated Neural Activation of Basal and Centromedial Amygdala}

\subsubsection{Anxiety- Associated cFOS Expression in Basal and Centromedial Amygdala}

While many of the amygdala nuclei are implicated in regulation of anxiety and fear behavior, most of the studies focused on the role of lateral amygdala (LA), BA, and lateral subdivision of central amygdala (CeL) in anxiety and fear related processing (Tovote et al. 2015; Janak \& Tye 2015). The neural activity of these nuclei was linked to behavioral manifestations of conditioned fear during various stages of the fear-conditioning paradigm, with relatively few studies describing neural correlates of unconditioned anxiety- related behavior, such as the one measured during the open field test. Therefore, I characterized the anxiety- associated neural activity of LA, BA, CeL, and medial subdivision of central amygdala (CeM) in Nlgn2 KO, IgSF9b KO, double KO and WT mice, and additionally two posterior sub regions of BA in Nlgn2 KO and WT mice (Figures 3.1.4, 3.2.4), by measuring the expression of neural activity marker cFOS (Schurr et al. 1985). Apart from BA and $\mathrm{CeM}$, the rest of the assayed regions did not show differential anxiety- associated cFOS induction in any of the genotypes. This finding demonstrates that although LA and CeL are strongly involved in acquisition and storage of conditioned fear (LeDoux 2003), they may play a lesser role in mediation of anxiety-related behaviors.

As might be expected from an anxiety-associated enhancement of BA-to-CeM projections in Nlgn2 KO mice, I observed a two-fold increase in cFOS expression in CeM in Nlgn2 KO mice, compared to WT mice (Figure 3.2.4). Strikingly, while Nlgn2 KO and double KO mice demonstrated similar overactivation of CeM-targeting excitatory projection neurons in BA, the anxiety- associated activation of CeM was completely rescued in double $\mathrm{KO}$ mice. Apart from the excitatory projections from $\mathrm{BA}, \mathrm{CeM}$ receives inhibitory projections from $\mathrm{CeL}$ that were shown to regulate CeM activity during fear- related processing (Ciocchi et al. 2010). While I did not quantify the cFOS activation specifically of the neurons that project from CeL to CeM yet, this is an ongoing experiment that hopefully will help to determine whether the suppression of neural 


\section{DISCUSSION}

activity of $\mathrm{CeM}$ in double $\mathrm{KO}$ mice may be a consequence of an increased inhibitory drive from CeL. Alternatively, as intrinsic inhibitory connections in the CeM exist (Jolkkonen \& Pitkanen 1998), the rescue of anxiety- associated activation of CeM may involve local increase in inhibitory transmission, such as identified in CeM of IgSF9b and double KO mice (Figure 3.2.20).

\subsubsection{Limitations of cFOS Assay}

While measuring cFOS expression provides an excellent quantitative estimate of neural activity during various behavioral states (Singewald et al. 2003; Knapska et al. 2007) and allows to simultaneously measure activity of several types of neurons, the cFOS assay has two major limitations, i.e. (1) the time-consuming nature of the current serial section-based methodology, and (2) the limited temporal resolution of the signal, which precludes direct correlation with behavioural responses. Below I discuss alternative approaches that I have implemented or initiated to overcome these limitations.

\subsubsection{Whole-brain functional imaging}

First, the cFOS assay is extremely time consuming, and due to its low throughput, neural activity of brain regions distal to amygdala, with the exception of medial prefrontal cortex, were not assessed in this study. However, additional brain regions such as BNST and ventral hippocampus are reciprocally interconnected with basal amygdala (Kim et al. 2013; Felix-Ortiz et al. 2013; Woodruff \& Sah 2007) and may therefore contribute to the differential activation of BA in Nlgn2 $\mathrm{KO}$, IgSF9b KO and double KO mice. To fully characterize the mechanisms underlying anxietyrelated phenotype of Nlgn2 $\mathrm{KO}$ mice and anxiety rescue in double $\mathrm{KO}$ mice, it would be necessary to assess the cFOS expression of the whole brain.

It recently became possible to generate brain-wide cFOS expression maps using iDISCO, a novel optical clearing technique that allows for immunolabelling and rapid imaging of the whole brain using light sheet microscopy (Renier et al. 2016). Together with Dr. Krueger- Burg and Dr. Camin 


\section{DISCUSSION}

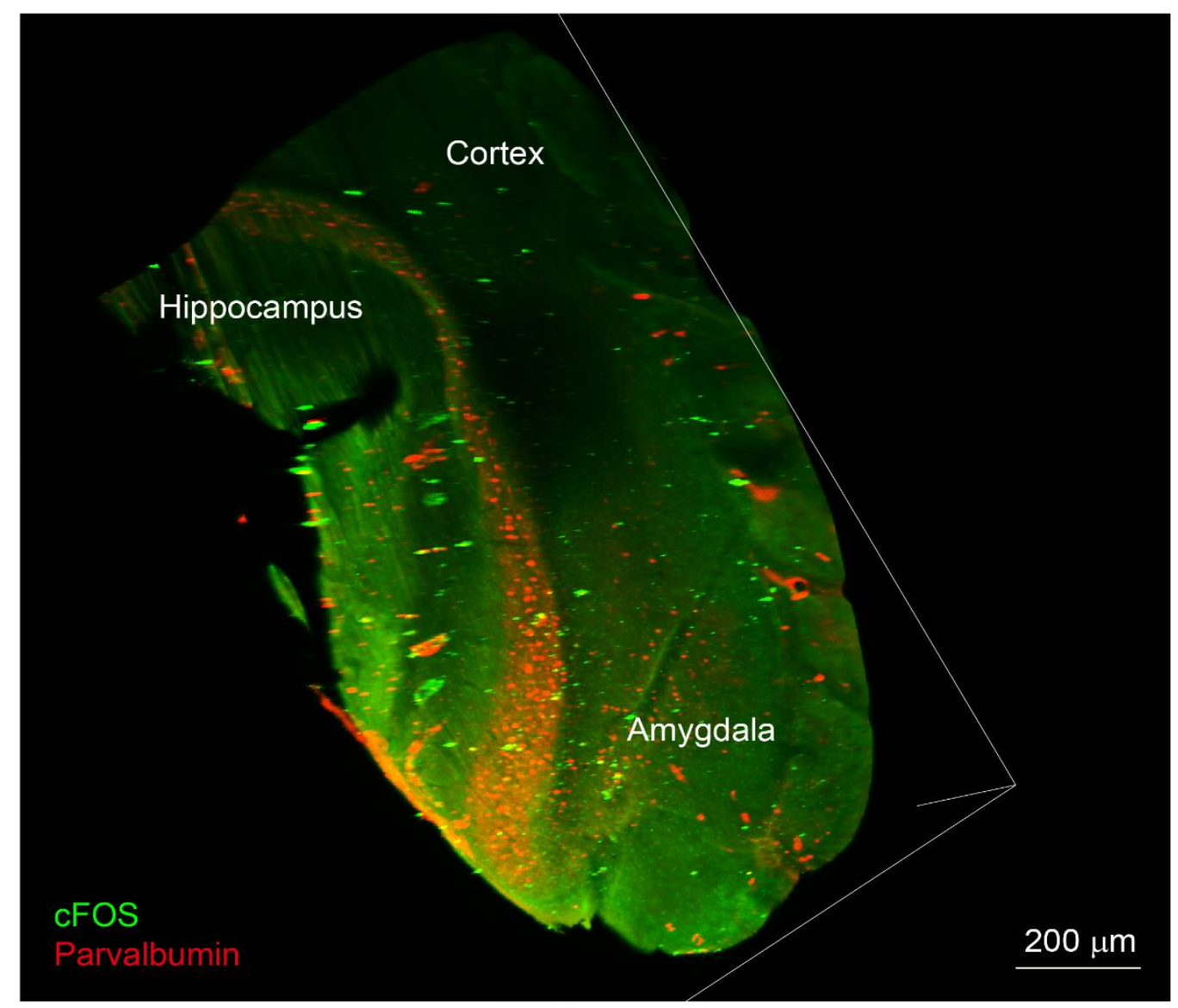

Figure 4.2| cFOS and PV immunolabeling of whole mouse brain combined with optical clearing using iDISCO protocol.

Dean, I implemented this technique and produced preliminary 3D image of amygdala and hippocampus labelled with cFOS and PV marker (Figure 4.2). Further application of iDISCO will help to fully map the brain regions that form the anxiety processing circuit in WT mice, as well as to characterize the role of $\mathrm{Nlgn} 2$ and $\mathrm{IgSF} 9 \mathrm{~b}$ in the regulation of distinct neuronal populations within these brain regions.

\subsubsection{In vivo Recording of Neural Activity in Freely Behaving Mice}

The second limitation of cFOS assay is that it lacks the temporary resolution necessary to assess dynamic changes in neural activity during behavioral tasks, such as exploration of the open field. Therefore, I initiated a collaboration with Dr. Hugo Cruces-Solis to complement quantification of 


\section{DISCUSSION}

cFOS expression with in vivo recordings of CeM while the mice explore the OF chamber. My primary motivation was to confirm that the rescue of neural activity in CeM, which I observed with the cFOS assay, correlates with the rescue of anxiety behavior. Additionally, I sought to characterize the contribution of the coordinated neural activity in CeM to the processing of aversive stimuli.

\subsubsection{Neural Activity of CeM During Exploration of the Open Field}

During exploration of the open field chamber, the neural activity of BA, including the firing of individual neurons and the local field potentials (LFPs) that correspond to coordinated firing of neuronal populations, increases as the mice transition into the center of the open field chamber (Likhtik et al. 2013). Therefore, neural activity of BA was postulated to differentially encode safety (the periphery of the open field) and danger (the center of the open field) (Stujenske et al. 2014; Likhtik et al. 2013). The danger- induced increase in neural firing of BA was proposed to activate $\mathrm{CeM}$, the primary output region of the BA, which mediates autonomic and behavioral responses associated with anxiety (Krettek \& Price 1978; Davis 1992; LeDoux et al. 1988). While characterizing the neural mechanisms underlying discrimination between safety and danger is one of the keys to understanding the pathophysiology of anxiety- related disorders, the neural activation of CeM during unconditioned behavioral manifestation of anxiety was never assessed.

Together with Dr. Hugo Cruces- Solis, I measured the LFP of CeM in WT, Nlgn2 KO, IgSF9b $\mathrm{KO}$ and double $\mathrm{KO}$ mice during exploration of the open field chamber. Our findings confirm that IgSF9b deletion normalizes the otherwise robustly enhanced neural activity of CeM in Nlgn2 KO mice in the open field. Moreover, we identified a specific frequency range of oscillatory activity in CeM, i.e. the high $\gamma$-frequency range (70 -120 Hz), which strongly correlates with the extent of avoidance of the open field center. Accordingly, "anxious" Nlgn2 KO mice demonstrate an increase in oscillatory activity at $70-120 \mathrm{~Hz}$ during exploration of the open field center, which is rescued in the double $\mathrm{KO}$ mice. Finally, we show that activity of CeM increases specifically during transition into the center, indicating that similarly to BA, CeM can encode safety and danger signals. 


\section{DISCUSSION}

As LFPs reflect the summary of excitatory and inhibitory synaptic inputs of a neural population (Mazzoni et al. 2012), it is possible that an increase in inhibitory synaptic transmission in CeM of double KO mice may contribute to the modulation of anxiety- related neural activity in CeM. Since GABAergic transmission in amygdala coordinates neural firing during behavioral responses to anxiogenic stimuli (Prager et al. 2016), proteins that regulate inhibitory synaptic function may play an important role in regulation of anxiety-associated neural activity.

\subsection{Conclusions: Implications for Molecular Mechanisms of Anxiety}

Anxiety is an essential part of normal behavioral responses to potentially threatening environmental stimuli. Nevertheless, excessive anxiety that translates into avoidance of nonthreating situations, thus interfering with daily life, is a pathological condition with a lifetime prevalence of up to $28 \%$ (Kessler, Demler, et al. 2005). Anxiety is typically treated with benzodiazepines that enhance the overall GABAergic transmission (Macdonald \& Barker 1978), but these drugs act non-specifically at the vast majority of inhibitory synapses in the central nervous system and hence cause a number of undesirable and potentially dangerous side effects. Therefore, there is a pressing need for detailed studies of the mechanisms underlying anxiety, with the goal of identifying novel therapeutic targets whose function is specific to brain regions, circuits and synaptic connections implicated in anxiety processing.

A model organism is needed to study the neural underpinnings of anxiety. My work identifies Nlgn2 KO mice as an excellent mouse model of anxiety that recapitulates the key mechanisms underlying anxiety in humans, including increased activation of amygdala during processing of aversive stimuli (Etkin \& Wager 2007), decreased GABAergic tone (Lydiard 2003) and excessive avoidance behavior (Bereza et al. 2009). Identification of Nlgn2 mutations in patients with schizophrenia and anxiety (Sun et al. 2011; Parente et al. 2016) further highlights the relevance of Nlgn2 to psychiatric conditions. Therefore, Nlgn2 KO mice offer construct validity and face validity and thus may provide insights into synaptic pathophysiology of anxiety, such as the findings presented in this work (summarized in Figure 4.4). 

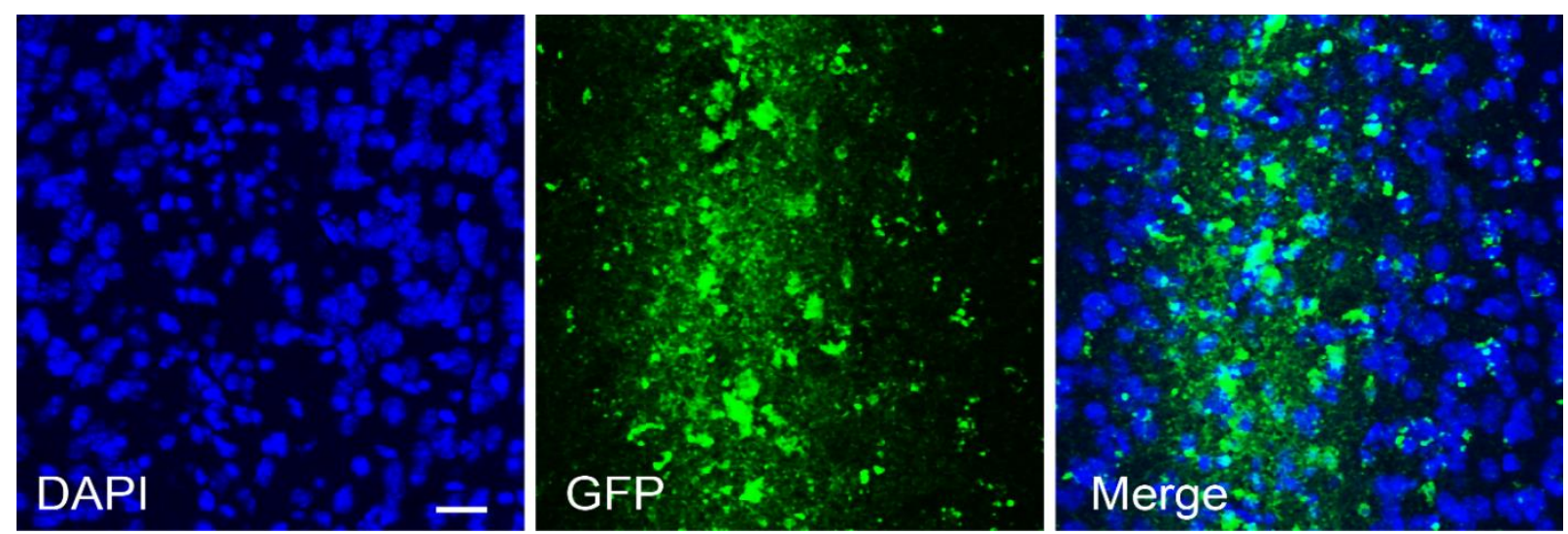

Figure 4.3| Viral delivery of GFP- Cre construct into basal amygdala. Scale bar, $20 \mu \mathrm{m}$.

Deletion of the novel synaptic protein IgSF9b rescues the enhanced anxiety phenotype of Nlgn2 KO mice. While further studies are needed for the detailed description of IgSF9b function in anxiety in vivo, I identified that deletion of IgSF9b normalizes the increased anxiety-related processing in centromedial amygdala of Nlgn2 KO mice. This finding indicates that both Nlgn2 and IgSF9b may play a crucial role in the modulation of anxiety behavior. Experiments to establish causality between IgSF9b deletion and the rescue of anxiety in Nlgn2 KO mice are underway. Virus-mediated delivery (Figure 4.3) of IgSF9b shRNA knockdown construct into basal and/or centromedial amygdala will address two important questions. First, it will determine whether IgSF9b acts to rescue anxiety in a specific brain region. Second, as IgSF9b knock down will take place in adult animals, it will determine whether anxiety-conferring aberrant neural activity can be corrected at post developmental stages.

I demonstrate here that both Nlgn2 and IgSF9b act at specific subpopulations of inhibitory synapses. Deletion of Nlgn2 affects synaptic transmission at perisomatic synapses surrounding excitatory neurons in BA, while deletion of IgSF9b affects perisomatic synapses onto inhibitory interneurons in CeM. By identifying proteins that regulate transmission at particular synaptic connections which are especially relevant to the generation of pathological anxiety behaviors, it may be possible to develop improved therapeutic strategies that target these synapses more specifically and are better suited to the treatment of at least a subset of anxiety disorders. Further experiments aimed to link Nlgn2 and IgSF9b to behavioral phenotypes related to psychiatric disorders may provide essential insights into underlying molecular mechanisms and potential drug targets for the treatment of these disorders. 


\section{DISCUSSION}

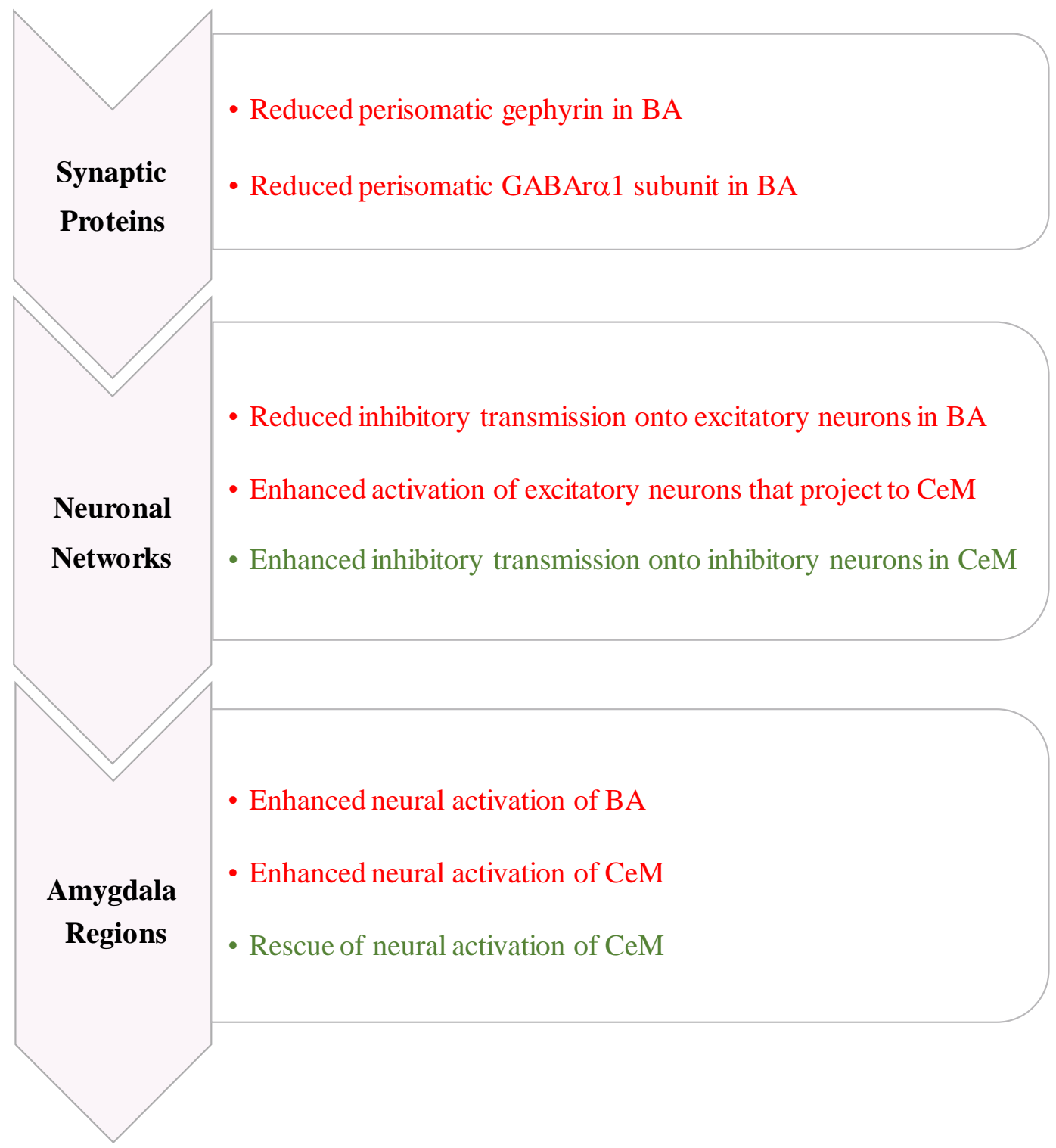

Effect of NIgn2 deletion

Effect of IgSF9b deletion in NIgn2 KO mice

Figure 4.4| Summary of the effects of Nlgn2 deletion and IgSF9b deletion on anxiety- processing circuit. 


\section{REFERENCES}

Adhikari, A. et al., 2015. Basomedial amygdala mediates top-down control of anxiety and fear. Nature.

Amaral, D.G., Schumann, C.M. \& Nordahl, C.W., 2008. Neuroanatomy of autism. Trends in Neurosciences, 31(3), pp.137-145.

Anderson, A.K. \& Phelps, E.A., 2001. Lesions of the human amygdala impair enhanced perception of emotionally salient events. Nature, 411(6835), pp.305-309.

Antonelli, R. et al., 2014. Pin1-dependent signalling negatively affects GABAergic transmission by modulating neuroligin2/gephyrin interaction. Nature communications, 5, p.5066.

Belligni, E.F. et al., 2012. $790 \mathrm{~Kb}$ microduplication in chromosome band 17p13.1 associated with intellectual disability, afebrile seizures, dysmorphic features, diabetes, and hypothyroidism. European Journal of Medical Genetics, 55(3), pp.222-224.

Bereza, B.G., Machado, M. \& Einarson, T.R., 2009. Systematic review and quality assessment of economic evaluations and quality-of-life studies related to generalized anxiety disorder. Clinical Therapeutics, 31(6), pp.1279-1308.

Bienvenu, T.C.M. et al., 2012. Cell-Type-Specific Recruitment of Amygdala Interneurons to Hippocampal Theta Rhythm and Noxious Stimuli In Vivo. Neuron, 74(6), pp.1059-1074.

Blanchard, D.C. \& Blanchard, R.J., 1972. Innate and Conditioned Reactions To Threat in Rats With Amygdaloid Lesions. Journal of Comparative and Physiological Psychology, 81(2), pp.281-290.

Blundell, J. et al., 2009. Increased anxiety-like behavior in mice lacking the inhibitory synapse cell adhesion molecule neuroligin 2. Genes, brain, and behavior, 8(1), pp.114-26.

Bourgeron, T., 2016. Current knowledge on the genetics of autism and propositions for future research. Comptes Rendus Biologies, 339(7), pp.300-307.

Braga, R.J., Reynolds, G.P. \& Siris, S.G., 2013. Anxiety comorbidity in schizophrenia. Psychiatry Research, 210(1), pp.1-7.

Budreck, E.C. \& Scheiffele, P., 2007. Neuroligin-3 is a neuronal adhesion protein at GABAergic and glutamatergic synapses. European Journal of Neuroscience, 26(7), pp.1738-1748.

Burkarth, N. et al., 2007. Neurofascin regulates the formation of gephyrin clusters and their subsequent translocation to the axon hillock of hippocampal neurons. Molecular and Cellular Neuroscience, 36(1), pp.59-70.

Buzsáki, G., Anastassiou, C. a. \& Koch, C., 2012. The origin of extracellular fields and currents - EEG, ECoG, LFP and spikes. Nature Reviews Neuroscience, 13(6), pp.407-420.

Carola, V. et al., 2002. Evaluation of the elevated plus-maze and open-field tests for the assessment of 
anxiety-related behaviour in inbred mice. Behavioural Brain Research, 134(1-2), pp.49-57.

Chubykin, A.A. et al., 2007. Activity-Dependent Validation of Excitatory versus Inhibitory Synapses by Neuroligin-1 versus Neuroligin-2. Neuron.

Chubykin, A.A. et al., 2005. Dissection of synapse induction by neuroligins: Effect of a neuroligin mutation associated with autism. Journal of Biological Chemistry.

Ciocchi, S. et al., 2010. Encoding of conditioned fear in central amygdala inhibitory circuits. Nature, 468(7321), pp.277-282.

Craig, A.M. \& Kang, Y., 2007. Neurexin-neuroligin signaling in synapse development. Current Opinion in Neurobiology, 17(1), pp.43-52.

Davis, M., 1992. THE ROLE OF THE AMYGDALA IN FEAR AND ANXIETY. Annu. Rev. Neurosci, 15 , pp.353-75.

Dean, C. et al., 2003. Neurexin mediates the assembly of presynaptic terminals. Nature Neuroscience, 6(7), pp.708-716.

Dean, C. \& Dresbach, T., 2006. Neuroligins and neurexins: Linking cell adhesion, synapse formation and cognitive function. Trends in Neurosciences, 29(1), pp.21-29.

Dixon, C. et al., 2014. GABA A Receptor $\alpha$ and $\gamma$ Subunits Shape Synaptic Currents via Different Mechanisms. Journal of Biological Chemistry, 289(9), pp.5399-5411.

Dong, N., Qi, J. \& Chen, G., 2007. Molecular reconstitution of functional GABAergic synapses with expression of neuroligin-2 and GABAA receptors. Molecular and Cellular Neuroscience.

Ehrlich, I. et al., 2009. Amygdala Inhibitory Circuits and the Control of Fear Memory. Neuron, 62(6), pp.757-771.

Essrich, C. et al., 1998. Postsynaptic clustering of major GABAA receptor subtypes requires the $\gamma 2$ subunit and gephyrin. Nature Neuroscience, 1(7), pp.563-571.

Etkin, A. et al., 2009. Disrupted Amygdalar Subregion Functional Connectivity and Evidence of a Compensatory Network in Generalized Anxiety Disorder. Archives of General Psychiatry, 66(12), p.1361.

Etkin, A. \& Wager, T.D., 2007. Functional neuroimaging of anxiety: A meta-ana lysis of emotional processing in PTSD, social anxiety disorder, and specific phobia. American Journal of Psychiatry, 164(10), pp.1476-1488.

Felix-Ortiz, A.C. et al., 2013. Neuron Report BLA to vHPC Inputs Modulate Anxiety-Related Behaviors. Neuron, 79, pp.658-664.

Feng, G., 1998. Dual Requirement for Gephyrin in Glycine Receptor Clustering and Molybdoenzyme Activity. Science, 282(5392), pp.1321-1324. Available at: http://www.sciencemag.org/cgi/doi/10.1126/science.282.5392.1321 [Accessed March 20, 2017]. 
Földy, C., Malenka, R.C. \& Südhof, T.C., 2013. Autism-associated neuroligin-3 mutations commonly disrupt tonic endocannabinoid signaling. Neuron, 78(3), pp.498-509.

Fritschy, J.-M. et al., 2006. Differential Dependence of Axo-Dendritic and Axo-Somatic GABAergic Synapses on GABAA Receptors Containing the 1 Subunit in Purkinje Cells. Journal of Neuroscience, 26(12), pp.3245-3255.

Fritschy, J.-M. \& Mohler, H., 1995. GABAA-receptor heterogeneity in the adult rat brain: Differential regional and cellular distribution of seven major subunits. The Journal of Comparative Neurology, 359(1), pp.154-194.

Fritschy, J.M., Panzanelli, P. \& Tyagarajan, S.K., 2012. Molecular and functional heterogeneity of GABAergic synapses. Cellular and Molecular Life Sciences, 69(15), pp.2485-2499.

Fu, Z. \& Vicini, S., 2009. Neuroligin-2 accelerates GABAergic synapse maturation in cerebellar granule cells. Molecular and cellular neurosciences, 42(1), pp.45-55.

Gauthier, I. \& Nuss, P., 2015. Anxiety disorders and GABA neurotransmission: a disturbance of modulation. Neuropsychiatric Disease and Treatment, Volume 11, p.165.

Gibson, J.R., Huber, K.M. \& Sudhof, T.C., 2009. Neuroligin-2 Deletion Selectively Decreases Inhibitory Synaptic Transmission Originating from Fast-Spiking but Not from Somatostatin-Positive Interneurons. Journal of Neuroscience, 29(44), pp.13883-13897.

Goldstein, P.A. et al., 2002. Prolongation of hippocampal miniature inhibitory postsynaptic currents in mice lacking the GABA(A) receptor alpha1 subunit. Journal of Neurophysiology, 88(6), pp.32083217.

Graf, E.R. et al., 2004. Neurexins induce differentiation of GABA and glutamate postsynaptic specializations via neuroligins. Cell.

Günther, U. et al., 1995. Benzodiazepine-insensitive mice generated by targeted disruption of the gamma 2 subunit gene of gamma-aminobutyric acid type A receptors. Proceedings of the National Academy of Sciences of the United States of America, 92(17), pp.7749-7753.

Hammer, M. et al., 2015. Perturbed Hippocampal Synaptic Inhibition and ??-Oscillations in a Neuroligin4 Knockout Mouse Model of Autism. Cell Reports, 13(3), pp.516-523.

Harrison, P.J. \& Weinberger, D.R., 2005. Schizophrenia genes, gene expression, and neuropathology: on the matter of their convergence. Molecular Psychiatry, 10(1), pp.40-68.

Herb, A. et al., 1992. The third y subunit of the y-aminobutyric acid type A receptor family (benzodiazepine receptor/cDNA cloning/electrophysiology/in situ hybridization). , 89, pp.14331437.

Hines, R.M. et al., 2008. Synaptic Imbalance, Stereotypies, and Impaired Social Interactions in Mice with Altered Neuroligin 2 Expression. Journal of Neuroscience, 28(24), pp.6055-6067. 
Hoon, M. et al., 2011. Neuroligin-4 is localized to glycinergic postsynapses and regulates inhibition in the retina. Proceedings of the National Academy of Sciences of the United States of America, 108(7), pp.3053-8.

Hoon, M. et al., 2009. Neuroligin 2 Controls the Maturation of GABAergic Synapses and Information Processing in the Retina. Journal of Neuroscience, 29(25).

Hu, X., Luo, J.H. \& Xu, J., 2015. The interplay between synaptic activity and neuroligin function in the CNS. BioMed Research International, 2015, pp.34-47.

Ichtchenko, K., Nguyen, T. \& Südhof, T.C., 1996. Structures, alternative splicing, and neurexin binding of multiple neuroligins. Journal of Biological Chemistry.

Iwata, J. et al., 1986. Intrinsic neurons in the amygdaloid field projected to by the medial geniculate body mediate emotional responses conditioned to acoustic stimuli. Brain Research, 383(1), pp.195-214.

Jamain, S. et al., 2003. Mutations of the X-linked genes encoding neuroligins NLGN3 and NLGN4 are associated with autism. Nature Genetics, 34(1), pp.27-29.

Janak, P.H. \& Tye, K.M., 2015. From circuits to behaviour in the amygdala. Nature, 517(7534), pp.284292.

Jedlicka, P. et al., 2011. Increased dentate gyrus excitability in neuroligin-2-deficient mice in vivo. Cerebral Cortex, 21(2), pp.357-367.

Jolkkonen, E.S. a \& Pitkanen, A., 1998. Intrinsic Connections of the Rat Amygdaloid Complex : Projections Originating in the Central Nucleus. the Journal of Comparative Neurology, 72(September 1997), pp.53-72.

Kemppainen, S. \& Pitkanen, A., 2000. Distribution of parvalbumin, calretinin, and calbindin-D28k immunoreactivity in the rat amygdaloid complex and colocalization with $\gamma$-aminobutyric acid. The Journal of Comparative Neurology, 426(3), pp.441-467. http://doi.wiley.com/10.1002/1096-

Kendler, K.S. et al., 1992. Major Depression and Generalized Anxiety Disorder. Archives of General Psychiatry, 49(9), p.716.

Kerti-Szigeti, K., Nusser, Z. \& Eyre, M.D., 2014. Synaptic GABAA Receptor Clustering without the 2 Subunit. Journal of Neuroscience, 34(31), pp.10219-10233.

Kessler, R.C., Berglund, P., et al., 2005. Lifetime Prevalence and Age-of-Onset Distributions of DSM-IV Disorders in the National Comorbidity Survey Replication. Archives of General Psychiatry, 62(6), p.593.

Kessler, R.C., Demler, O., et al., 2005. Prevalence and Treatment of Mental Disorders, 1990 to 2003. New England Journal of Medicine, 352(24), pp.2515-2523.

Kim, S.-Y. et al., 2013. Diverging neural pathways assemble a behavioural state from separable features in anxiety. 
Kins, S., Betz, H. \& Kirsch, J., 2000. Collybistin, a newly identified brain-specific GEF, induces submembrane clustering of gephyrin. Nature neuroscience, 3(1), pp.22-9.

Knapska, E. et al., 2007. Functional Internal Complexity of Amygdala: Focus on Gene Activity Mapping After Behavioral Training and Drugs of Abuse. Physiological Reviews, 87(4).

Kneussel, M., Brandstatter, J., et al., 2001. Gephyrin-independent clustering of postsynaptic GABA(A) receptor subtypes. Molecular and cellular neurosciences, 17(6), pp.973-982.

Kneussel, M., Helmut Brandstätter, J., et al., 2001. Gephyrin-Independent Clustering of Postsynaptic GABAA Receptor Subtypes. Molecular and Cellular Neuroscience, 17(6), pp.973-982.

Kneussel, M. et al., 1999. Loss of postsynaptic GABA(A) receptor clustering in gephyrin-deficient mice. The Journal of neuroscience : the official journal of the Society for Neuroscience, 19(21), pp.928997.

Kohl, C. et al., 2015. Hippocampal neuroligin-2 links early-life stress with impaired social recognition and increased aggression in adult mice. Psychoneuroendocrinology, 55, pp.128-143.

Kohl, C. et al., 2013. Hippocampal Neuroligin-2 Overexpression Leads to Reduced Aggression and Inhibited Novelty Reactivity in Rats P. Campolongo, ed. PLoS ONE, 8(2), p.e56871.

De Koninck, Y. \& Mody, I., 1994. Noise analysis of miniature IPSCs in adult rat brain slices: properties and modulation of synaptic GABAA receptor channels. Journal of neurophysiology, 71(4), pp.1318-35.

van der Kooij, M.A. et al., 2014. Impaired Hippocampal Neuroligin-2 Function by Chronic Stress or Synthetic Peptide Treatment is Linked to Social Deficits and Increased Aggression. Neuropsychopharmacology, 39(5), pp.1148-1158.

Kralic, J.E. et al., 2002. Molecular and Pharmacological Characterization of GABA A Receptor $\alpha 1$ Subunit Knockout Mice. The Journal of pharmacology and experimental therapeutics, 302(3), pp.1037-1045.

Krettek, J.E. \& Price, J.L., 1978. Amygdaloid projections to subcortical structures within the basal forebrain and brainstem in the rat and cat. The Journal of Comparative Neurology, 178(2), pp.225253.

Krueger, D.D. et al., 2012. The role of neurexins and neuroligins in the formation, maturation, and function of vertebrate synapses. Current Opinion in Neurobiology, 22(3), pp.412-422.

Lange, M.D. et al., 2014. Glutamic Acid Decarboxylase 65: A Link Between GABAergic Synaptic Plasticity in the Lateral Amygdala and Conditioned Fear Generalization. Neuropsychopharmacology, 39(9), pp.2211-2220.

Lebow, M. \& Chen, A., 2016. Overshadowed by the amygdala: the bed nucleus of the stria terminalis emerges as key to psychiatric disorders. 
LeDoux, J., 2003. The emotional brain, fear, and the amygdala. Cellular and Molecular Neurobiology, 23(4-5), pp.727-738.

LeDoux, J.E. et al., 1988. Different projections of the central amygdaloid nucleus mediate autonomic and behavioral correlates of conditioned fear. The Journal of neuroscience : the official journal of the Society for Neuroscience, 8(7), pp.2517-29.

LeDoux, J.E. et al., 2017. The birth, death and resurrection of avoidance: a reconceptualization of a troubled paradigm. Molecular Psychiatry, 22(1), pp.24-36.

Lévi, S. et al., 2002. Dystroglycan Is Selectively Associated with Inhibitory GABAergic Synapses But Is Dispensable for Their Differentiation. Journal of Neuroscience, 22(11).

Lewis, D.A., Hashimoto, T. \& Volk, D.W., 2005. Cortical inhibitory neurons and schizophrenia. Nature Reviews Neuroscience, 6(4), pp.312-324.

Liang, J. et al., 2015. Conditional neuroligin-2 knockout in adult medial prefrontal cortex links chronic changes in synaptic inhibition to cognitive impairments. Molecular Psychiatry, 20, pp.850-859.

Likhtik, E. et al., 2013. Prefrontal entrainment of amygdala activity signals safety in learned fear and innate anxiety. Nature Neuroscience, 17(1), pp.106-113.

Lüthi, A. \& Lüscher, C., 2014. Pathological circuit function underlying addiction and anxiety disorders. Nature Neuroscience, 17(12), pp.1635-1643.

Lydiard, R.B., 2003. The role of GABA in anxiety disorders. The Journal of clinical psychiatry, 64 Suppl 3, pp.21-7.

Macdonald, R.L. \& Barker, J.L., 1978. Different actions of anticonvulsant and anesthetic barbiturates revealed by use of cultured mammalian neurons. Science (New York, N.Y.), 200(4343), pp.775-7.

Macdonald, R.L. \& Olsen, R.W., 1994. GABAA RECEPTOR CHANNELS. Annu. Rev. Neurosci, 17, pp.569-602.

Marowsky, A., Fritschy, J.-M. \& Vogt, K.E., 2004. Functional mapping of GABA A receptor subtypes in the amygdala. The European journal of neuroscience, 20(5), pp.1281-1289.

Mascagni, F. \& McDonald, A.J., 2003. Immunohistochemical characterization of cholecystokinin containing neurons in the rat basolateral amygdala. Brain research, 976(2), pp.171-84.

Mcdonald, A.J., Mascagni, F. \& Guo, L., 1996. Projections of the medial and lateral prefrontal cortices to the amygdala: A Phaseolus vulgaris leucoagglutinin study in the rat. Neuroscience, 71(1), pp.55-75.

Mitra, P.P. \& Bokil, H.S., 2007. Observed Brain Dynamics.

Müller, I., Çalışkan, G. \& Stork, O., 2015. The GAD65 knock out mouse - a model for GABAergic processes in fear- and stress-induced psychopathology. Genes, Brain and Behavior, 14(1), pp.37-45.

Muller, J.F., Mascagni, F. \& McDonald, A.J., 2007. Postsynaptic targets of somatostatin-containing interneurons in the rat basolateral amygdala. The Journal of Comparative Neurology, 500(3), 
pp.513-529. 2017].

Muller, J.F., Mascagni, F. \& McDonald, A.J., 2006. Pyramidal cells of the rat basolateral amygdala: synaptology and innervation by parvalbumin-immunoreactive interneurons. The Journal of comparative neurology, 494(4), pp.635-50.

Namburi, P. et al., 2015. A circuit mechanism for differentiating positive and negative associations. Nature, 520(7549), pp.675-678.

Nguyen, Q.-A. et al., 2016. Distinct roles for extracellular and intracellular domains in neuroligin function at inhibitory synapses. eLife, 5, pp.781-796.

Niehoff, D.L. \& Kuhar, M.J., 1983. Benzodiazepine receptors: localization in rat amygdala. The Journal of neuroscience: the official journal of the Society for Neuroscience, 3(10), pp.2091-7.

Ogawa, Y. \& Rasband, M.N., 2008. The functional organization and assembly of the axon initial segment. Current Opinion in Neurobiology, 18(3), pp.307-313.

Olmos-Serrano, J.L. et al., Cellular/Molecular Defective GABAergic Neurotransmission and Pharmacological Rescue of Neuronal Hyperexcitability in the Amygdala in a Mouse Model of Fragile X Syndrome.

Ortinski, P.I. et al., 2004. Expression of Distinct Subunits of GABAA Receptor Regulates Inhibitory Synaptic Strength. Journal of Neurophysiology, 92(3), pp.1718-1727.

Otis, T.S., De Koninck, Y. \& Modyt, I., 1994. Lasting potentiation of inhibition is associated with an increased number of y-aminobutyric acid type A receptors activated during miniature inhibitory postsynaptic currents (hippocampus/gule cell/dentate gyms/kindling/epilepsy). Neurobiology, 91, pp.7698-7702.

Panzanelli, P. et al., 2011. Distinct mechanisms regulate GABAA receptor and gephyrin clustering at perisomatic and axo-axonic synapses on CA1 pyramidal cells. The Journal of physiology, 589(20), pp.4959-80.

Parente, D.J. et al., 2016. Neuroligin 2 nonsense variant associated with anxiety, autism, intellectual disability, hyperphagia, and obesity. American Journal of Medical Genetics, Part A.

Patel, T.P. et al., 2014. An open-source toolbox for automated phenotyping of mice in behavioral tasks. Frontiers in behavioral neuroscience, 8, p.349.

Petty, F. \& Sherman, A.D., 1984. Plasma GABA levels in psychiatric illness. Journal of Affective Disorders, 6(2), pp.131-138.

Pikkarainen, M. et al., 1999. Projections from the lateral, basal, and accessory basal nuclei of the amygdala to the hippocampal formation in rat. The Journal of Comparative Neurology, 403(2), pp.229-260.

Poulopoulos, A. et al., 2009. Neuroligin 2 Drives Postsynaptic Assembly at Perisomatic Inhibitory 
Synapses through Gephyrin and Collybistin. Neuron.

Prager, E.M. et al., 2016. The basolateral amygdala $\gamma$-aminobutyric acidergic system in health and disease. Journal of Neuroscience Research, 94(6), pp.548-567.

Renier, N. et al., 2016. Mapping of Brain Activity by Automated Volume Analysis of Immediate Early Genes. Cell, 165(7), pp.1789-1802.

Rodgers, R.J. \& Dalvi, A., 1997. Anxiety, defence and the elevated plus-maze. In Neuroscience and Biobehavioral Reviews. pp. 801-810.

Romanski, L.M. et al., 1993. Somatosensory and auditory convergence in the lateral nucleus of the amygdala. Behavioral Neuroscience, 107(3), pp.444-450.

Rothwell, P.E. et al., 2014. Autism-associated neuroligin-3 mutations commonly impair striatal circuits to boost repetitive behaviors. Cell, 158(1), pp.198-212.

Rubenstein, J.L.R. \& Merzenich, M.M., 2003. Model of autism: increased ratio of excitation/inhibition in key neural systems. Genes, brain, and behavior, 2(5), pp.255-67.

Sagar, S., Sharp, F. \& Curran, T., 1988. Expression of c-fos protein in brain: metabolic mapping at the cellular level. Science, 240(4857).

Sah, P. et al., The Amygdaloid Complex: Anatomy and Physiology.

Saha, R. et al., 2017. GABAergic Synapses at the Axon Initial Segment of Basolateral Amygdala Projection Neurons Modulate Fear Extinction. Neuropsychopharmacology, 42(2), pp.473-484.

Sanders, S.K. \& Shekhar, A., 1991. Blockade of GABA A receptors in the region of the anterior basolateral amygdala of rats elicits increases in heart rate and blood pressure*. Brain Research, 576, pp.101-110. 00000aab0f6c\&acdnat=1489397506_281622b7c1919a1ec612936e470ca80e [Accessed March 13, 2017].

Sanders, S.K. \& Shekhar, A., 1995. Regulation of anxiety by GABAA receptors in the rat amygdala. Pharmacology, biochemistry, and behavior, 52(4), pp.701-6.

Schiess, M.C., Callahan, P.M. \& Zheng, H., 1999. Characterization of the electrophysiological and morphological properties of rat central amygdala neurons in vitro. Journal of Neuroscience Research, 58(5), pp.663-673.

Schurr, a et al., 1985. Expression of c-fos Protein in Brain: Metabolic Mapping at the Cellular Level. Brain Res. Brain Res. Bull. Brain Res, 701(10), pp.39-44.

Sheng, M., 1996. PDZs and Receptor/Channel Clustering: Rounding Up the Latest Suspects. Neuron, 17(4), pp.575-578.

Shi, S.-H. et al., 2004. From The Cover: Control of dendrite arborization by an Ig family member, dendrite arborization and synapse maturation 1 (Dasm1). Proceedings of the National Academy of Sciences, 101(36), pp.13341-13345. 
Shyn, S.I. et al., 2011. Novel loci for major depression identified by genome-wide association study of Sequenced Treatment Alternatives to Relieve Depression and meta-analysis of three studies. Molecular Psychiatry, 16(2), pp.202-215.

Silverman, J.L. et al., 2013. Developmental delays and reduced pup ultrasonic vocalizations but normal sociability in mice lacking the postsynaptic cell adhesion protein neuroligin2. Behavioural Brain Research, 251, pp.50-64.

Singewald, N., Salchner, P. \& Sharp, T., 2003. Induction of c-Fos expression in specific areas of the fear circuitry in rat forebrain by anxiogenic drugs. Biological Psychiatry, 53(4), pp.275-283.

Smith, T.A., 2001. Type A gamma-aminobutyric acid (GABAA) receptor subunits and benzodiazepine binding: significance to clinical syndromes and their treatment. British journal of biomedical science, 58(2), pp.111-21.

Sotres-Bayon, F. et al., 2012. Gating of Fear in Prelimbic Cortex by Hippocampal and Amygdala Inputs. Neuron, 76(4), pp.804-812.

Sotres-Bayon, F., Bush, D.E.A. \& LeDoux, J.E., 2004. Emotional perseveration: an update on prefrontalamygdala interactions in fear extinction. Learning \& memory (Cold Spring Harbor, N.Y.), 11(5), pp.525-35.

Spampanato, J., Polepalli, J. \& Sah, P., 2011. Interneurons in the basolateral amygdala. Neuropharmacology, 60(5), pp.765-773.

Stujenske, J.M. et al., 2014. Fear and safety engage competing patterns of theta-gamma coupling in the basolateral amygdala. Neuron, 83(4), pp.919-33.

Südhof, T.C., 2008. Neuroligins and neurexins link synaptic function to cognitive disease. Nature, 455(7215), pp.903-911.

Südhof, T.C., 2008. Neuroligins and neurexins link synaptic function to cognitive disease. Nature, 455(7215), pp.903-11.

Sumita, K. et al., 2007. Synaptic scaffolding molecule (S-SCAM) membrane-associated guanylate kinase with inverted organization (MAGI)-2 is associated with cell adhesion molecules at inhibitory synapses in rat hippocampal neurons. Journal of Neurochemistry, 100(1), pp.154-166.

Sun, C. et al., 2011. Identification and functional characterization of rare mutations of the neuroligin-2 gene (NLGN2) associated with schizophrenia. Human Molecular Genetics, 20(15), pp.3042-3051.

Sur, C. et al., 2001. Loss of the major GABA(A) receptor subtype in the brain is not lethal in mice. The Journal of neuroscience : the official journal of the Society for Neuroscience, 21(10), pp.3409-18.

Taschenberger, H., Scheuss, V. \& Neher, E., 2005. Release kinetics, quantal parameters and their modulation during short-term depression at a developing synapse in the rat CNS. The Journal of physiology, 568(Pt 2), pp.513-37. 
Tovote, P., Fadok, J.P. \& Lüthi, A., 2015. Neuronal circuits for fear and anxiety. Nature Reviews Neuroscience, 16(6), pp.317-331.

Tretter, V. et al., 2008. The clustering of $\mathrm{GABA}(\mathrm{A})$ receptor subtypes at inhibitory synapses is facilitated via the direct binding of receptor alpha 2 subunits to gephyrin. The Journal of neuroscience : the official journal of the Society for Neuroscience, 28(6), pp.1356-1365.

Tye, K.M. et al., 2011. Amygdala circuitry mediating reversible and bidirectional control of anxiety. Nature, 471(7338), pp.358-362.

Uchida, N. et al., 1996. The catenin/cadherin adhesion system is localized in synaptic junctions bordering transmitter release zones. The Journal of cell biology, 135(3), pp.767-79.

Varoqueaux, F. et al., 2006. Neuroligins Determine Synapse Maturation and Function. Neuron.

Varoqueaux, F., Jamain, S. \& Brose, N., 2004. Neuroligin 2 is exclusively localized to inhibitory synapses. European Journal of Cell Biology, 83(9), pp.449-456.

Vicini, S. et al., 2001. GABA(A) receptor alpha1 subunit deletion prevents developmental changes of inhibitory synaptic currents in cerebellar neurons. The Journal of neuroscience : the official journal of the Society for Neuroscience, 21(9), pp.3009-16.

Vislay, R.L. et al., 2013. Homeostatic Responses Fail to Correct Defective Amygdala Inhibitory Circuit Maturation in Fragile X Syndrome. Journal of Neuroscience, 33(17).

Vogel, E. et al., 2016a. Projection-Specific Dynamic Regulation of Inhibition in Amygdala MicroCircuits. Neuron, 91(3), pp.644-651.

Vogel, E. et al., 2016b. Projection-Specific Dynamic Regulation of Inhibition in Amygdala MicroCircuits. Neuron, 91(3), pp.644-651.

Weiskrantz, L., 1956. Behavioral changes associated with ablation of the amygdaloid complex in monkeys. Journal of comparative and physiological psychology, 49(4), pp.381-91.

Whiting, P.J. et al., 1999. Molecular and Functional Diversity of the Expanding GABA-A Receptor Gene Family. Annals of the New York Academy of Sciences, 868(1 MOLECULAR AND), pp.645-653.

Wiltgen, B.J. et al., 2009. The $\alpha 1$ subunit of the GABA(A) receptor modulates fear learning and plasticity in the lateral amygdala. Frontiers in Behavioral Neuroscience, 3, p.37.

Wolff, S.B.E. et al., 2014. Amygdala interneuron subtypes control fear learning through disinhibition. Nature, 509.

Woo, J. et al., 2013. The adhesion protein IgSF9b is coupled to neuroligin 2 via S-SCAM to promote inhibitory synapse development. Journal of Cell Biology, 201(6), pp.929-944.

Woodruff, A.R. \& Sah, P., 2007. Inhibition and Synchronization of Basal Amygdala Principal Neuron Spiking by Parvalbumin-Positive Interneurons. Journal of Neurophysiology, 98(5), pp.2956-2961.

Woodruff, A.R. \& Sah, P., 2007. Networks of parvalbumin-positive interneurons in the basolateral 
amygdala. The Journal of neuroscience : the official journal of the Society for Neuroscience, 27(3), pp.553-63.

Yamaguchi, Y. et al., 2002. Glycobiology of the synapse: the role of glycans in the formation, maturation, and modulation of synapses. Biochimica et biophysica acta, 1573(3), pp.369-76.

Ye, X. et al., 2016. Direct dorsal hippocampal-prelimbic cortex connections strengthen fear memories. Nature Neuroscience, 20(1), pp.52-61.

Zhang, B. et al., 2015. Neuroligins Sculpt Cerebellar Purkinje-Cell Circuits by Differential Control of Distinct Classes of Article Neuroligins Sculpt Cerebellar Purkinje-Cell Circuits by Differential Control of Distinct Classes of Synapses. , pp.781-796.

Zoghbi, H.Y., 2003. Postnatal neurodevelopmental disorders: meeting at the synapse? Science (New York, N.Y.), 302(5646), pp.826-30.

Zoghbi, H.Y. \& Bear, M.F., 2012. Synaptic Dysfunction in Neurodevelopmental Disorders Associated with Autism and Intellectual Disabilities. Cold Spring Harbor Perspectives in Biology, 4(3) 


\section{List of figures}

Figure 1.1| Serial processing of sensory information in BLA-CeA circuit............................................. 3

Figure 1.2| BLA long-range connections in regulation of diverse behavioral domains............................. 4

Figure 1.3| Microcircuit in BA that mediates fear- related behavior. ...................................................... 6

Figure $1.4 \mid$ Synaptic interactions of Nlgn2 at inhibitory synapses........................................................ 11

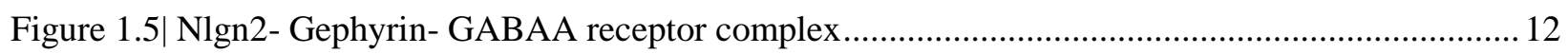

Figure 1.6 Interaction of IgSF9b and Nlgn2 at the post synaptic membrane .......................... 18

Figure 1.7| Framework for studying the role of Nlgn2 and IgSF9b in anxiety- related brain regions........ 19

Figure 2.1| Stages of in vivo electrophysiology experiment .................................................................24

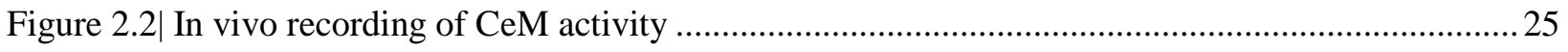

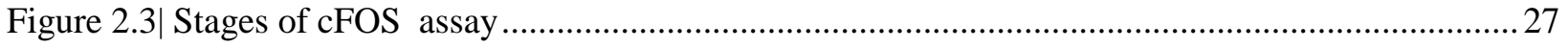

Figure 2.4| Stages in quantification of synaptic markers. ..................................................................29

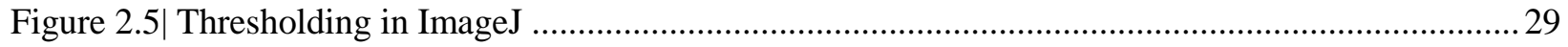

Figure 2.6| Colocalization of cFOS positive neurons with cellular markers in IMARIS .......................... 30

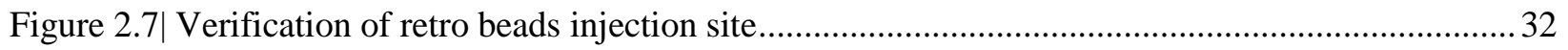

Figure 3.1.1| Anxiety phenotype and locomotor activity of Nlgn2 KO mice ........................................43

Figure 3.1.2| Horizontal activity of Nlgn2 KO mice during anxiety testing .......................................... 44

Figure 3.1.3| Activity of Nlgn2 KO mice in home cage setting.......................................................... 46

Figure 3.1.4| Anxiety-induced cFOS expression in basolateral amygdala of Nlgn2 KO mice..................47

Figure 3.1.5| Anxiety induced cFOS expression in cortical components of anxiety circuitry...................47

Figure 3.1.6| Basal cFOS expression in anterior BA of WT and Nlgn2 KO mice...................................48

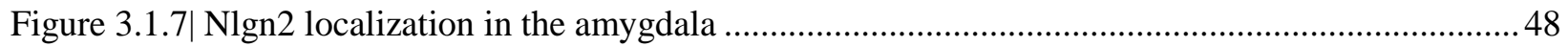

Figure 3.1.8| Localization and expression levels of gephyrin in BA of Nlgn2 KO mice .........................50

Figure 3.1.9| Localization and expression levels of presynaptic inhibitory markers in BA of Nlgn2 KO mice .51

Figure 3.1.10| Effect of Nlgn2 deletion on inhibitory synaptic transmission in BA and CeA...................52

Figure 3.1.11| Effect of Nlgn2 deletion on cellular components of the anxiety circuitry in BA. ..............54

Figure 3.2.1| Anxiety phenotype and locomotor activity of Nlgn2 KO, IgSF9b KO and double KO mice in

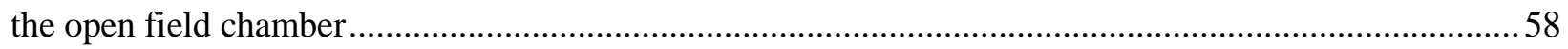

Figure 3.2.2 Anxiety phenotype of Nlgn2 KO, IgSF9b KO and double KO mice in the intermediate zone and extended center of the open field .59

Figure 3.2.3 Anxiety phenotype of female Nlgn2 KO, IgSF9b KO and double KO mice in the open field chamber. 
Figure 3.2.4| Anxiety-associated cFOS expression in distinct nuclei of amygdala in WT, Nlgn2 KO, IgSF9b $\mathrm{KO}$ and double $\mathrm{KO}$ mice

Figure 3.2.5 Effect of IgSF9b deletion on anxiety- associated activation of inhibitory network in basal amygdala in WT and Nlgn2 KO mice.

Figure 3.2.6| Effect of Nlgn2 and IgSF9b KO on anxiety- associated activation of glutamatergic_neurons in basal amygdala that project to centromedial amygdala. .66

Figure 3.2.7| Effect of exposure to the open field on CeM neural activity in WT, Nlgn2 KO, IgSF9b KO and double KO. 67

Figure 3.2.8| CeM activity during exploration of periphery and center of the OF. .68

Figure 3.2.9| Effect of Nlgn2 $\mathrm{KO}$ on CeM activity during center-periphery transitions and correlation of CeM activity to anxiety- like behavior. .70

Figure 3.2.10| IgSF9b expression in the brain. .72

Figure 3.2.11| IgSF9b expression and colocalization with synaptic markers in basal amygdala. .73

Figure 3.2.12| Colocalization of IgSF9b with $\gamma 2$ subunit of GABA receptor in centromedial amygdala compared to colocalization of Nlgn2 with inhibitory markers. .74

Figure 3.2.13| Amygdala expression of inhibitory post synaptic partners of Nlgn2 and IgSF9b 75 Figure 3.2.14|Effect of Nlgn2 and IgSF9b deletion on composition of perisomatic GABA receptors in basal and centromedial amygdala. 76

Figure 3.2.15| Effect of Nlgn2 and IgSF9b deletion on perisomatic expression of gephyrin in basal amygdala. .78

Figure 3.2.16| Effect of Nlgn2 and IgSF9b deletion on perisomatic expression of post synaptic scaffold proteins in centromedial amygdala .80

Figure 3.2.17| Overall expression of post synaptic partners of Nlgn2 and IgSF9b in Nlgn2 KO, IgSF9b KO and double $\mathrm{KO}$ mice. 82

Figure 3.2.18| Total number of perisomatic synapses and VGAT/Vglut2 ratio in basal amygdala of Nlgn2 $\mathrm{KO}, \mathrm{IgSF} 9 \mathrm{~b} \mathrm{KO}$ and double KOs. .83

Figure 3.2.19| Effect of Nlgn2 KO and IgSF9b KO on inhibitory synaptic transmission in basal amygdala.

Figure 3.2.20| Effect of Nlgn2 KO and IgSF9b KO on inhibitory synaptic transmission in centromedial amygdala. 85

Figure 3.2.21| Expression of VGAT and Calbindin in centromedial nucleus. .87

Figure 4.1| Colocalization of Gephyrin and AIS marker Ankyrin G in Nlgn2 KO and WT mice..... 94

Figure 4.2| cFOS and PV immunolabeling of whole mouse brain combined with optical clearing using iDISCO protocol. 105 
Figure 4.3| Viral delivery of GFP- Cre construct into basal amygdala .................................................. 108 Figure 4.4| Summary of the effects of Nlgn2 deletion and IgSF9b deletion on anxiety-processing circuit. 


\section{List of tables}

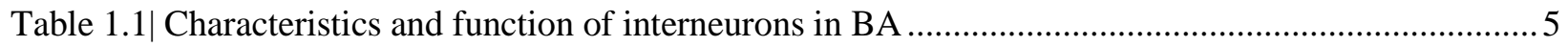

Table 1.2| Summary of electrophysiological characterization of Nlgn2 KO ......................................... 14

Table 1.3| Summary of behavioral deficits in Nlgn2 in comparison to WTs........................................... 17

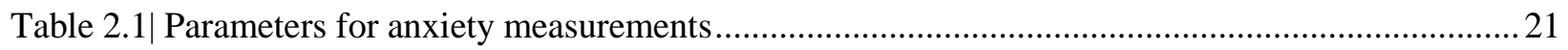

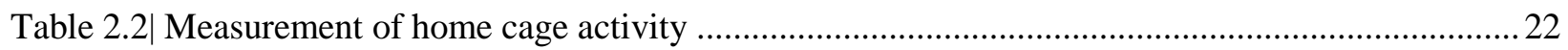

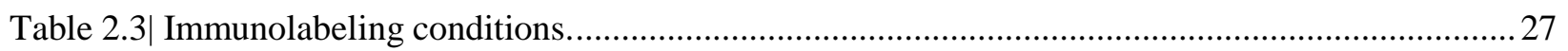

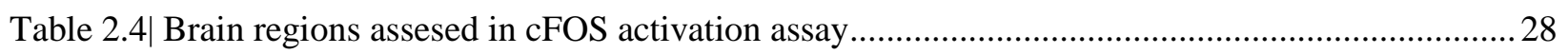

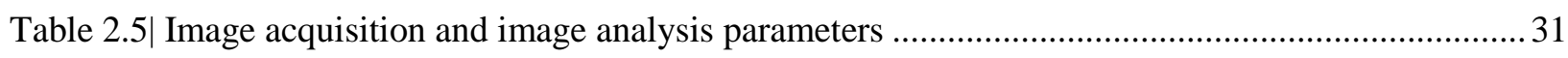

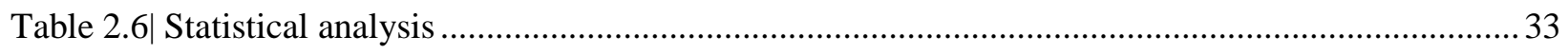

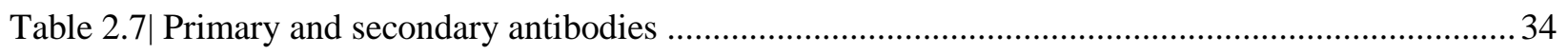

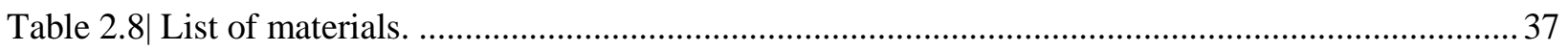

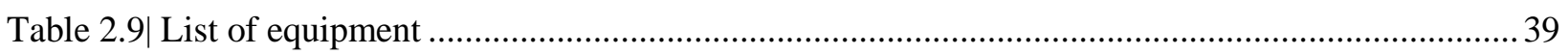

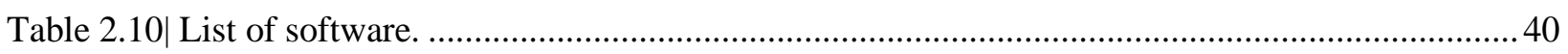

Table 3.2.1| Effect of Nlgn2 KO and IgSF9b KO on molecular composition of inhibitory synapses in basal

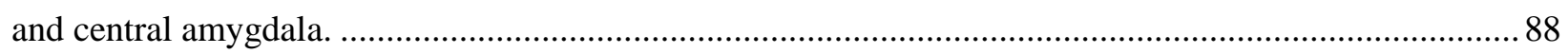




\section{OLGA BABAEV}

Max Planck Institute of Experimental Medicine Department of Molecular Neurobiology

Hermann- Rein Strasse 3, 37035

Göttingen, Germany
Email: babaev@em.mpg.de

Lab Phone: $\quad$ +49 (0) 5513899685

Lab Fax: $\quad$ +49 (0) 5513899715

\section{Education}

Ph.D., Neuroscience

Expected April 2017

University of Göttingen, Göttingen, Germany

Master of Science, Neuroscience

September 2013

University of Göttingen, Göttingen, Germany

Grade: 1.2 (on a scale from 1 to 5, with 1 being the highest), top student of the class

Bachelor, Pharmacy (summa cum laude)

June 2011

Ben Gurion University, Beer Sheva, Israel

Grade: 93 (on scale from 1 to 100), valedictorian

\section{Research Experience}

Graduate research with Prof. Nils Brose

April 2013-Present

Max Planck Institute of Experimental Medicine, Göttingen, Germany

Project 1: Functional and Molecular Deficits Underlying Increased Anxiety-Related Phenotype in Neuroligin 2 Knock Out Mice

- Planned and performed tests of anxiety-related behavior in mice

- Established quantitative analysis of synaptic proteins visualized by confocal microscopy

- Established cFOS activation assay to measure anxiety-induced activation of distinct neuronal populations in amygdala

Project 2: Molecular and Circuit Mechanism Underlying the Rescue of Anxiety Related Phenotype in Double Neuroligin 2 IgSF9b Knock Out Mice

- Established in vivo retrograde tracing to identify target structures of projection neurons in basal amygdala

- Tested an optical clearing protocol that allows 3D visualization of brain structures colabeled with cFOS and neuronal markers

- Initiated a collaboration to learn and subsequently perform in vivo recording of local field potentials in basal amygdala of mice during open field test

Project 3: Role of Neuroligin 2 in Anxiety Processing Circuit

- Established in vivo stereotaxic viral delivery into basal amygdala to delete Neuroligin 2 specifically in basal amygdala 
Undergraduate research with Prof. Nils Brose

September 2012- April 2013

Max Planck Institute of Experimental Medicine, Göttingen, Germany

- Performed behavioral screening and initial molecular characterization of Neuroligin 2 and IgSF9b knock out mice

Internship in Research and Development

May-July 2012

Sartorius- Stedim Biotech, Göttingen, Germany

- Optimized protein microarray chips for application in in vitro diagnostics tests

Visiting student

December 2011-February 2012

Max Planck Institute of Experimental Medicine, Göttingen, Germany

- Expressed IgF1 receptor in neuronal cultures to study its signaling pathway

- Gained experience with DNA cloning techniques

Research assistant

July 2008-August 2011

Ben Gurion University, Beer Sheva, Israel

- Recorded cortical field potentials from slices

- Established an enzymatic assay to measure the activity of nitric oxide synthase in brain homogenates

- Analyzed protein expression with western blotting, immunoprecipitation and high performance liquid chromatography

\section{Awards and Honors}

1. Travel grant, German Neuroscience Society (2016)

2. Three-year doctoral fellowship, Minerva Foundation (2013).

3. Israel Pharmacy Union Award for Academic Excellence (2009).

4. Academic honors from Faculty of Health Sciences, Ben-Gurion University $(2010,2007)$.

\section{Publications}

1. Olga Babaev, Paolo Botta, Elisabeth Meyer, Christian Müller, Hannelore Ehrenreich, Nils Brose, Andreas Lüthi, Dilja Krueger-Burg. Neuroligin 2 Deletion Alters Inhibitory Synapse Function and Anxiety-Associated Neuronal Activation in the Amygdala. Neuropharmacology, 2016, 100:56-65

2. Hugo Cruces-Solís, Zhizi Jing, Olga Babaev, Nicola Strenzke, Livia de Hoz. Differential midbrain processing of predictable and random sounds in unsupervised learning. Under Review 


\section{Stipends}

1. Stipend of Max- Planck Society for international Ph.D. students (April 2013- November 2013).

2. Stipend of the Excellence Foundation for the Promotion of the Max Planck Society (September 2011- September 2012).

\section{Societies}

1. European Behavioral Pharmacology Society (since 2015).

2. Israel Society for Neuroscience (since 2014).

\section{Talks and Poster Presentations}

1. German Neuroscience Society Meeting, March 24, 2017 (Talk)

2. Wolffson Institute for Biomedical Research, University College London, June $8^{\text {th }}, 2016$ (Seminar)

3. European Brain and Behavior Society and European Behavioral Pharmacology Society Joint Meeting, September 12-15, 2015 (Talk).

4. Gordon Research Seminar and Conference "Amygdala in Health and Disease", August 1-7, 2015 (Poster). Served as a discussion leader.

5. Neurizons 2015, $6^{\text {th }}$ Biennial Neuroscience Conference, Göttingen, Germany, May 26-29, 2015 (Poster).

6. $11^{\text {th }}$ Meeting of German Neuroscience Society, March 18-21 (Poster).

7. $9^{\text {th }}$ FENS Forum of Neuroscience, July 5-9, 2014 (Poster).

8. Neurizons 2013, $5^{\text {th }}$ Biennial Neuroscience Conference, May 22-25, 2013 (Poster).

\section{Organization of conferences}

1. Women's Careers and Networks Symposium, Göttingen, Germany (February 2016).

2. Neurizons 2015, $6^{\text {th }}$ Biennial Neuroscience Conference, Göttingen, Germany (May 2015). 